\title{
Neutrino Factory Targets and the MICE Beam
}

\author{
Kenneth Andrew Walaron \\ University of Glasgow
}

Thesis submitted for the degree of

Doctor of Philosophy

at the University of Glasgow

(c) K.A Walaron 2007 


\begin{abstract}
The future of particle physics in the next 30 years must include detailed study of neutrinos. The first proof of physics beyond the Standard Model of particle physics is evident in results from recent neutrino experiments which imply that neutrinos have mass and flavour mixing. The Neutrino Factory is the leading contender to measure precisely the neutrino mixing parameters to probe beyond the Standard Model physics. Significantly, one must look to measure the mixing angle $\theta_{13}$ and investigate the possibility of leptonic $\mathrm{CP}$ violation. If found this may provide a key insight into the origins of the matter/antimatter asymmetry seen in the universe, through the mechanism of leptogenesis.

The Neutrino Factory will be a large international multi-billion dollar experiment combining novel new accelerator and long-baseline detector technology. Arguably the most important and costly features of this facility are the proton driver and cooling channel. This thesis will present simulation work focused on determining the optimal proton driver energy to maximise pion production and also simulation of the transport of this pion flux through some candidate transport lattices. Bench-marking of pion crosssections calculated by MARS and GEANT4 codes to measured data from the HARP experiment is also presented.

The cooling channel aims to reduce the phase-space volume of the decayed muon beam to a level that can be efficiently injected into the accelerator system. The Muon Ionisation Cooling Experiment (MICE) hosted by the Rutherford Appleton laboratory, UK is a proof-of-principle experiment aimed at measuring ionisation cooling. The experiment will run parasitically to the ISIS accelerator and will produce muons from pion decay. The MICE beamline provides muon beams of variable emittance and momentum to the MICE experiment to enable measurement of cooling over a wide range of beam conditions. Simulation work in the design of this beamline is presented in this thesis as are results from an experiment to estimate the flux from the target into the beamline acceptance
\end{abstract}


To mum and dad... 


\section{Acknowledgements}

There are many people to whom I am indebted to for helping me produce this thesis and equally as important, keeping me sane. Firstly I would like to thank my supervisor Dr Paul Soler for his guidance. From day one it has been plentiful and always useful. I would also like to thank Mr Stephen Brooks, Dr Paul Drumm, Dr Malcolm Ellis, Dr Bill Murray, Dr Tom Roberts and Mr Kevin Tilley.

And now for those people, in no real order, who helped me stay clear of straight jackets. I would like to thank the "old school" i.e. Richard "Francis, Cuthbert, Cecil, bartholemew, Sakamoto, Rawk Cinema" Codling, David "Snyde-rat, top of the elevator" Petrie, Bob "Radagast the Brown, Jakey-Bob, Top Gun" Taylor, Neil "The Mole, The Ride" Thompson, John "The Fool, Hand of the Hippo, Columbo" Veitch and last but definitely not least Helen "Masai Bison, C, I like shoes and talking" McGlone. You made it possible. I would also like to acknowledge "The lunchtime crew" some of who have awesome chat and others who aided the completion of this thesis, reducing the amount of time spent at lunch, by having some of the most soul destroying geeky chat possible.

Next I would like to thank my office mates Dan "Lord of Root" Clements, you saved many a computer being lobbed out of the window, Andrew "Linford, im pure east coastlike, Im addicted to Tetris" Laing and Kenny "I have a cool name, my highscore is better than Andrews" Wraight. The last two tried to get me hooked on Scientific Linux Tetris while writing up and it nearly worked.

Now for my fellow MICE'ers. Aron "I cannae do it, pies" Fish, Chris "Dodgy beard" Rogers and Rikard "Mass of a tree, nature boy, I am completely mental" Sandstroem. Cheers guys.

Lastly, I would like to thank my Mum, Dad, David and rest of my family for their support. 


\section{Authors Declaration}

The work presented here is my own unless otherwise stated. The Neutrino Factory target simulations for all GEANT4 hadronic engines presented in Chapter 3 are my own work as is the analysis. The MARS data sets are contributed by S.J Brooks. Similarly with the benchmarking of GEANT4 and MARS codes to HARP data, presented in Chapter 4, the GEANT4 datasets and analysis was carried out by the author, the MARS data sets were contributed by S.J Brooks. My contribution to the target test experiment detailed in Chapter 6 was the construction of the unshielded detector, operating the detector during shift and the full analysis of the data collected by both detectors. The design of the MICE beamline presented in Chapter 7 is also my own work. Specifically the G4Beamline simulations and analysis following initial optics code designs carried out by K. Tilley. 


\section{Contents}

1 Neutrino Physics $\quad 2$

1.1 Discovery of the neutrino . . . . . . . . . . . . . . . 2

1.2 Neutrino Oscillation Theory . . . . . . . . . . . . . . . . . . 4

1.2.1 Three flavour oscillation in vacuum . . . . . . . . . . . . . 4

1.2.2 Neutrino Oscillations in matter . . . . . . . . . . . . . 8

1.3 Neutrino Phenomenology . . . . . . . . . . . . . . . . . . . . . 10

1.4 Atmospheric Neutrino Experiments . . . . . . . . . . . . . . . . . 11

1.5 Solar Neutrino Experiments . . . . . . . . . . . . . . . . . . 13

1.6 Reactor Neutrino Experiments . . . . . . . . . . . . . . . . . . . 19

1.7 Accelerator Neutrino Experiments . . . . . . . . . . . . . . . . 22

1.8 Conclusion . . . . . . . . . . . . . . . . . . . . . 29

2 The Neutrino Factory $\quad 31$

2.1 Introduction . . . . . . . . . . . . . . . . . . . 31

2.2 Neutrino Factory Design . . . . . . . . . . . . . . . . . . . 31

2.2.1 Proton Driver . . . . . . . . . . . . . . . . . 31

2.2.2 Target and Pion Capture . . . . . . . . . . . . . . . . 32

2.2.3 Muon Phase Rotation and Cooling . . . . . . . . . . . . . . 33

2.2.4 Muon Acceleration . . . . . . . . . . . . . . . . . 34

2.2 .5 Storage . . . . . . . . . . . . . . . . 34

2.3 Physics at a Neutrino Factory . . . . . . . . . . . . . . . . . 34

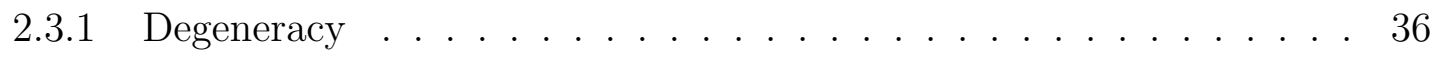

2.3.2 Near Detector . . . . . . . . . . . . . . . . . . 38

2.3.3 Optimal performance at a Neutrino Factory . . . . . . . . . . . 39

2.4 Conclusion . . . . . . . . . . . . . . . . . . . . 43

3 Target Studies at a Neutrino Factory $\quad 44$

3.1 Introduction . . . . . . . . . . . . . . . . . . . . 44

3.2 GEANT4 simulation and comparison with MARS . . . . . . . . . . . . 47

3.3 Pion yields as a function of incident proton energy . . . . . . . . . . . . . . 49

3.4 Comparison between GEANT4 hadronic models . . . . . . . . . . . . . . . 51

3.5 Pion angular distribution . . . . . . . . . . . . . . . . 54

3.6 Pion yields inside $20^{\circ}$ transverse acceptance . . . . . . . . . . . . . . . 56

3.7 Pion yields within $100 \mathrm{MeV}$ transverse momentum acceptance . . . . . . . 57

3.8 Pion yields in a neutrino factory front-end simulation . . . . . . . . . . . 58

3.8.1 Chicane/Linac pion decay and transport channel . . . . . . . . . . 59

3.8.2 Phase rotation pion decay and transport channel . . . . . . . . . 59 
3.9 Hadronic model comparison of transmission . . . . . . . . . . . . . . 61

3.10 Conclusion . . . . . . . . . . . . . . . . . . . 63

4 Benchmarking of GEANT4 and MARS to HARP data 66

4.1 Purpose of HARP: Introduction . . . . . . . . . . . . . . . 66

4.2 HARP Experimental Setup . . . . . . . . . . . . . . . . 66

4.3 Simulation . . . . . . . . . . . . . . . . . . . 67

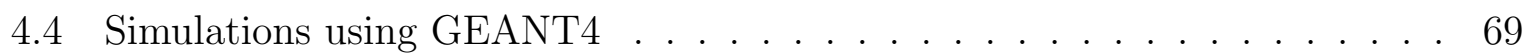

4.5 Calculation of differential cross-section . . . . . . . . . . . . . . 70

4.6 Harp positive pion double-differential cross-section results summary . . . . 72

4.7 GEANT4 double-differential cross-section $d^{2} \sigma^{\pi_{H A R P}^{+}} / d p d \Omega$ results . . . . . . 74

4.8 Results of comparison with MARS production cross-sections . . . . . . . . 78

4.9 Total Cross-sections . . . . . . . . . . . . . . . . . . 82

4.10 Conclusions . . . . . . . . . . . . . . . . . . 82

5 The Muon Ionisation Cooling Experiment (MICE) 85

5.1 Introduction . . . . . . . . . . . . . . . . . . . 85

5.2 Theory and definition of emittance . . . . . . . . . . . . . . . 87

5.3 Muon Cooling . . . . . . . . . . . . . . . . . . . . 91

5.4 Simulation . . . . . . . . . . . . . . . . . . . . . . . . . . . . . . . . . . . . . . .

5.5 Particle Detection and Kinematic Measurement . . . . . . . . . . . . . 94

5.5.1 Time-of-flight Detectors . . . . . . . . . . . . . . . 95

5.5.2 Tracking Detectors . . . . . . . . . . . . . . . 96

5.5.3 Downstream Electron-Muon Separation . . . . . . . . . . . . 97

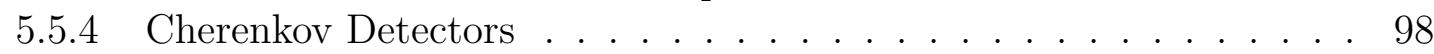

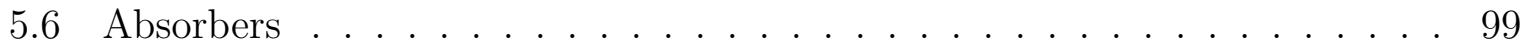

5.7 RF Cavities . . . . . . . . . . . . . . . . . . . . 101

5.8 MICE Magnetic Channel . . . . . . . . . . . . . . . . . . . . . . 102

5.9 Conclusion . . . . . . . . . . . . . . . . . . 103

6 Studies of particle production from the prototype MICE target 104

6.1 Introduction . . . . . . . . . . . . . . . . . 104

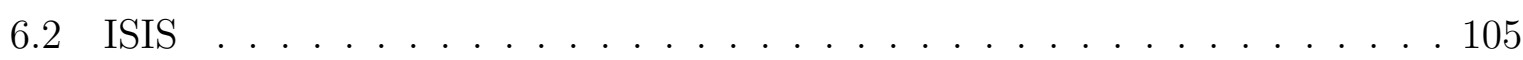

6.3 MICE target . . . . . . . . . . . . . . . . . . . . 107

6.4 Target test setup . . . . . . . . . . . . . . . . . . . . . . . . . . . . . . . . . . . . . . . . . . . . . . . .

6.5 Unshielded counters . . . . . . . . . . . . . . . . . . . 110

6.6 Shielded counters . . . . . . . . . . . . . . . . . . . 111

6.7 Data acquisition . . . . . . . . . . . . . . . . . . . . . . . . . . . . . . . . . . . . .

6.8 Simulations . . . . . . . . . . . . . . . . . . . . . 112

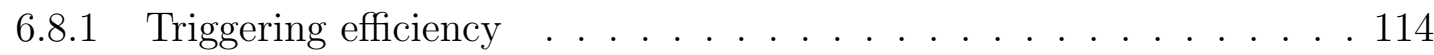

6.9 Detector Analysis . . . . . . . . . . . . . . . . . . . . 119

6.9.1 Event Selection . . . . . . . . . . . . . . . . . 119

6.9.2 Results of investigation of SP7 measured beam loss as a function of particle yields . . . . . . . . . . . . . . . . 124

6.9.3 Comparison of singles per proton on target from simulation and data128

6.9.4 Studies of particle production into the MICE beamline as a function of target position . . . . . . . . . . . . . . 130 
6.10 Conclusions . . . . . . . . . . . . . . . . . . . 131

7 MICE Beamline Design 133

7.1 Introduction . . . . . . . . . . . . . . . . . . . 133

7.2 Goals for the beamline . . . . . . . . . . . . . . . . . . . 133

7.3 The MICE target . . . . . . . . . . . . . . . . . . 135

7.4 MICE target normalisation . . . . . . . . . . . . . . . . . . . . . . . . . . . . . . . . . . . . . . . . . . .

7.5 Description of the beamline . . . . . . . . . . . . . . . . 138

7.6 Optical configuration of beamline . . . . . . . . . . . . . . . . . 142

7.7 Iteration of G4Beamline and TURTLE simulations . . . . . . . . . . . . 143

7.7.1 Calibration of optical code materials to GEANT4 material description 146

7.8 G4Beamline simulation . . . . . . . . . . . . . . . . . . . 149

7.8.1 Implementation of magnetic elements . . . . . . . . . . . . . . . 149

7.8.2 Implementation of beamline detectors . . . . . . . . . . . . . . . . . 151

7.8.3 Implementation of non-detector materials in beamline . . . . . . . . 152

7.9 Analysis of a $200 \mathrm{MeV} / \mathrm{c}$ muon beamline for the MICE experiment . . . . . 154

7.9 .1 Mid-emittance $200 \mathrm{MeV} / \mathrm{c}$ tune . . . . . . . . . . . . . . 155

7.9.2 Large emittance $200 \mathrm{MeV} / \mathrm{c}$ tune . . . . . . . . . . . . . . . 159

7.9.3 Low $\pi 200 \mathrm{MeV} / \mathrm{c} \ldots \ldots \ldots \ldots 2$

7.10 A typical example of cooling in MICE . . . . . . . . . . . . . . . . . . . . . . . . . . . . . . . . .

7.11 Conclusions . . . . . . . . . . . . . . . . . 167

8 Conclusions $\quad 169$ 


\section{List of Figures}

1.1 Energy distribution curve for the beta spectrum of Radium E [2] . . . . . . . . 4

1.2 Oscillation induced 3D rotation of flavour basis with respect to the mass basis [5] 6

1.3 The possible neutrino mass-hierarchies assuming no sterile neutrinos. Also shown are approximate flavour content of each mass eigenstate $[6] \ldots$. . . . . . . 6

1.4 Neutral-current and charged-current neutrino-electron scattering diagrams . . . 9

1.5 Super-Kamiokande zenith angle distributions for fully-contained 1-ring events. The points show data and the box histograms show Monte Carlo events assuming no oscillations. The solid line shows the best-fit expectation for $\nu_{\mu} \longleftrightarrow \nu_{\tau}$ oscillations with $\sin ^{2} 2 \theta=1.00$ and $\Delta m^{2}=2.1 \times 10^{-3} \mathrm{eV}^{2}[10] . . . . . .$.

1.6 Allowed oscillation parameter space for 2-flavour $\nu_{\mu} \longleftrightarrow \nu_{\tau}$ oscillations obtained from Super-Kamiokande $[10]$. . . . . . . . . . . . . . . . .

1.7 Ratio of the measured Super-Kamiokande data [11] to the Monte Carlo events without oscillation (points) as a function of reconstructed L/E together with the best-fit expectation for 2-flavour $\nu_{\mu} \longleftrightarrow \nu_{\tau}$ oscillations (solid black line). Also shown are the best-fit expectation for neutrino decay (dashed blue line) and decoherence (red dotted line). The errors are statistical. . . . . . . . . . . 14

1.8 Solar PP cycle reaction chain with branching fractions . . . . . . . . . . . . 15

1.9 Standard Solar Model (SSM) fluxes for solar neutrinos produced from different reaction branches $[12] \ldots \ldots \ldots$. . . . . . . . . . . . . 16

$1.10 \cos \left(\theta_{\text {sun }}\right)$ distribution of solar neutrino event candidates from the SuperKamiokande I data set (1496 days). [18] . . . . . . . . . . . . . . .

1.11 Top upper: The flux of $\mu+\tau$ neutrinos versus flux of electron neutrinos. CC, $\mathrm{NC}$ and ES fluxes are indicated by solid bands. The ${ }^{8} B \mathrm{SSM}$ solar flux is indicated by the dashed lines. The narrow band parallel to the SNO ES result corresponds to the Super-Kamiokande result. Right upper and lower: Global neutrino oscillation analysis using only solar neutrino data (a) and including KamLAND 766 ton-year data (b). Left lower: Charged current $T_{\text {eff }}$ spectrum with the best fit LMA parameters. A $1 \sigma$ uncertainty on the undistorted ${ }^{8} B$ model shape determined by detector systematics is indicated by the green band. The predicted spectrum is normalised to the same number of counts as the data spectrum [20]. . . . . . . . . . . . . . . . . . . . . . . .

1.12 (a) Allowed region in oscillation parameter space from KamLAND anti-neutrino data (shaded regions) and solar neutrino experiments (lines). (b) Result of combined two-flavour oscillation analysis of KamLAND and the observed solar fluxes under the assumption of CPT invariance. The fit gives $\Delta m^{2}=7.9_{-0.5}^{+0.6} \times$ $10^{-5}$ and $\tan ^{2} \theta=0.40_{-0.07}^{+0.10}[21] . \ldots \ldots \ldots . \ldots . \ldots 20 . \ldots \ldots$ 
1.13 Event distribution in $L_{0} / \mathrm{E}$, where $L_{0}$ is taken to be $180 \mathrm{~km}$. The solid blue curve is the best fit oscillation spectrum. The two remaining curves show the dependence on $L_{0} / \mathrm{E}$ for neutrino decay and decoherence theories [19]. . . . . .

1.14 Upper bound on $\sin ^{2}\left(2 \theta_{13}\right)$ from the CHOOZ experiment at $90 \%$ (dashed) CL and $3 \sigma$ (solid) CL for $1 \mathrm{DOF}$ as a function of $\Delta m_{31}^{2}$. The light (dark) shaded

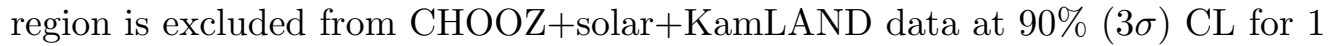
DOF. The horizontal lines indicate the current best-fit value and the $3 \sigma$ allowed

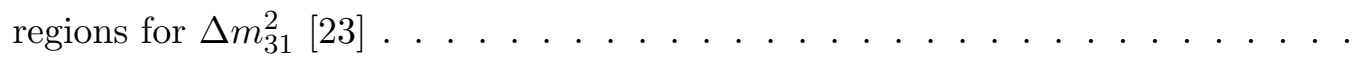

1.15 The ratio of measured to expected $\bar{\nu}_{e}$ flux from reactor experiments. The solid circle denotes the KamLAND result plotted at the flux averaged distance of approximately $180 \mathrm{~km}$. The shaded region indicates the flux prediction corresponding to the $95 \%$ CL LMA region parameter space from a global analysis of solar neutrino data. The dotted line is representive of a best-fit LMA prediction $\left(\sin ^{2} 2 \theta=0.833\right.$ and $\Delta m^{2}=5.5 \times 10^{-5} \mathrm{eV}^{2}$ The dashed line is the expected flux

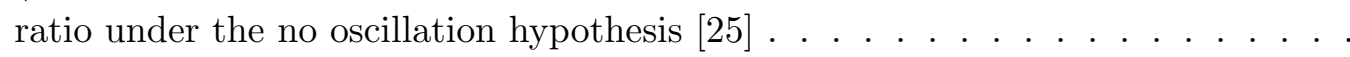

1.16 The upper plot shows the number of candidate $\nu_{e}$ events as a function of $E_{\nu}^{Q E}$. The points represent the data with statistical error, whereas the histogram shows the expected background with systematic errors from all sources. The vertical dashed line shows the threshold used in the two-neutrino oscillation analysis. Also shown are the best-fit oscillation spectrum (dashed histogram) and the background contributions from $\nu_{\mu}$ and $\nu_{e}$ events. The bottom panel shows the number of events with the predicted background subtracted as a function of $E_{\nu}^{Q E}$, where the points represent the data with total errors and the two histograms correspond to LSND solutions at high and low $\Delta m^{2}[27]$. . . . . . . . . . . .

1.17 Atmospheric parameter confidence levels from the MINOS, K2K and Super-

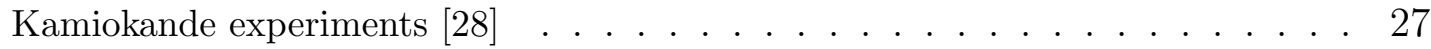

1.18 Schematic representation of the baseline beta-beam facility at the CERN SPS [33] 28

1.19 Summary of current experimental limits on neutrino oscillation parameters [35] . 30

2.1 Baseline Neutrino Factory design from The International Scoping Study. . . . . 32

2.2 Degenerate solutions of $\theta_{13}$ and $\delta$ for one neutrino charge conjugate (left). Degenerate solutions of $\theta_{13}$ and $\delta$ for neutrinos (solid line) and anti-neutrinos(dashed line) showing the remaining intrinsic clone degenerate solution(left)[43] . . . . .

2.3 Highlighting unique solution by observing same oscillation channel measures at two L/E values (left). Highlighting unique solution by observing silver and golden oscillation channels measured at one value of $\mathrm{L} / \mathrm{E}$ (right)[43] . . . . . .

2.4 The relative sensitivity to $\sin ^{2} 2 \theta_{13}$ compared to the optimal (white). The contours represent regions with a factor of $0.5,1,2,5,10$ above the optimal sensitivity. Each plot is produced by taking into account statistics, systematics, correlations and degeneracies. The optimal sensitivities are $\sin ^{2} 2 \theta_{13}<$ $1.4 \times 10^{-5}$ (statistics), $2.8 \times 10^{-5}$ (systematics), $2.4 \times 10^{-4}$ (correlations), $5.0 \times$ $10^{-4}$ (degeneracies) obtained at the energy and baselines displayed [45]. . . . . .

2.5 Maximal CP-violation sensitivity expressed as the reach in $\sin ^{2} 2 \theta_{13}$ at $\delta_{C P}=$ $\pi / 2$ and $3 \pi / 2$ for different $L$ and $E$. $\sin ^{2} 2 \theta_{13}$ reach is given at the $3 \sigma$ CL including correlations and degeneracies. The diamonds mark the minima which are chosen to be $\sin ^{2} 2 \theta_{13}=8.8 \times 10^{-5}$ for left panel and $\sin ^{2} 2 \theta_{13}=1.3 \times 10^{-3}$ for right panel $[45] . \ldots \ldots \ldots \ldots \ldots$ 
2.6 Plots show the sensitivity to a normal mass hierarchy for different values of the $\mathrm{CPV}$ phase $\delta_{C P}$ as a function of $\mathrm{L}$ and $\mathrm{E}$. The absolute reach in $\sin ^{2} 2 \theta_{13}$ at $3 \sigma$ including correlations and degeneracies is used as a figure of merit. The minima are at from left to right $\sin ^{2} 2 \theta_{13}=1.8 \times 10^{-4}, \sin ^{2} 2 \theta_{13}=6.7 \times 10^{-5}$ and $\sin ^{2} 2 \theta_{13}=1.6 \times 10^{-4}$ respectively [45] . . . . . . . . . . . . . . . .

2.7 Signal efficiencies for $\mu^{+}$(left) and $\mu^{-}[46]$ for a golden channel measurement using a magnetised calorimeter as described . . . . . . . . . . . . . . . .

2.8 Summary of the optimised performance of a neutrino for mass hierarchy (left panel), CP violation (middle panel) and $\mathrm{CP}$ violation with matter uncertainties included (right panel) at $3 \sigma \mathrm{CL}$. The matter uncertainty is $2 \%$ and the coloured regions are as detailed on the plot. The dashed line is a reference beta-beam[45].

3.1 Schematic layout of the proposed UK NUFACT target scheme. The target toroid (blue) has been shown as solid but could be segmented in $20 \mathrm{~cm}$ lengths. The dimensions of the toroid are such that it has a $1 \mathrm{~cm}$ radius with the distance between the rotational axis (middle of donut) to torus around $6 \mathrm{~m} . .$. . . . .

3.2 Open GL visualisation of the target geometry. The target is coloured red with the virtual detector volume coloured yellow. Protons are red, $\pi^{+}$green, $\pi^{-}$grey, $\mu^{+}$blue and all neutral particles are coloured cyan (almost all are neutrons). . . 48

3.3 Total $\pi^{ \pm}$yields . . . . . . . . . . . . . . . . . . . . . . 50

3.4 Pion per proton*Energy $(\mathrm{GeV})$ variations between GEANT4 and MARS. . . . . 51

3.5 Comparison between GEANT4 physics use cases in the range $2.2-10 \mathrm{GeV}$. . . . 52

3.6 Pion angular distribution with incident proton energy of $2.2 \mathrm{GeV}$. X-axis is cosine of the opening angle made between the outgoing pion and the $\mathrm{z}$-axis of the target 53

3.7 Pion angular distribution with incident proton energy of $15 \mathrm{GeV}$. X-axis is the cosine of the opening angle made between the outgoing pion and the $\mathrm{z}$-axis of the target . . . . . . . . . . . . . . . . . 54

3.8 Plot of angles (in degrees) containing $25 \%, 50 \%$ and $75 \%$ of pion distribution . . 55

3.9 Pion per (Proton*Energy $(\mathrm{GeV})$ ) within $20^{\circ}$ cone . . . . . . . . . . . . . 56

3.10 Pion per (Proton*Energy $(\mathrm{GeV})$ ) within $20^{\circ}$ cone and $\mathrm{Pt}<100 \mathrm{MeV}$. . . . . . . 58

$3.11 \pi^{+} / \pi^{-}$fate along $2.2 \mathrm{GeV}$ optimised Chicane/Linac Channel, $P_{\text {long }}$ vs $P_{\text {transv }}$ [58] 60

$3.12 \pi^{+} / \pi^{-}$transmission along $2.2 \mathrm{GeV}$ optimised Chicane/Linac Channel using a GEANT4 QGSP model. . . . . . . . . . . . . . . . . . 60

$3.13 \pi^{+} / \pi^{-}$fate along $2.2 \mathrm{GeV}$ optimised Phase Rotator Channel, $P_{\text {long }}$ vs $P_{\text {transv }}[58] 61$

$3.14 \pi^{+} / \pi^{-}$transmission along $2.2 \mathrm{GeV}$ optimised Phase Rotation Channel using a GEANT4 QGSP model . . . . . . . . . . . . . . . . . 62

3.15 Comparison between GEANT4 hadronic model use-cases for $\pi^{+} / \pi^{-}$transmission along 2.2GeV optimised Chicane/Linac Channel . . . . . . . . . . . . . . . 63

3.16 Comparison between GEANT4 hadronic model use-cases for $\pi^{+} / \pi^{-}$transmission along 2.2GeV optimised Phase Rotation Channel . . . . . . . . . . . . . . . . 64

3.17 Percentage difference in "beam-power-normalised" transmission between Chicane/Linac and Phase Rotator channels. Percentage normalised to Chicane/Linac transmission . . . . . . . . . . . . . . . . . . . . . 64

4.1 The layout of the HARP experiment . . . . . . . . . . . . . . . . 68

4.2 The HARP beam-trigger layout . . . . . . . . . . . . . . . . . . . . . . 68 
4.3 Measured double-differential $\pi^{+}$production cross-section $d^{2} \sigma /(d p d \Omega)$ for incoming protons of $12.9 \mathrm{GeV} / \mathrm{c}$ on an aluminium target as a function of pion momentum $\mathrm{p}$ for pion polar angle $30 \leq \theta<60 \mathrm{mrad}$. The results of GEANT4 simulations with different hadronic models are compared to the HARP experimental data . . . . . . . . . . . . . . . . . . . . . . .

4.4 Measured double-differential $\pi^{+}$production cross-section $d^{2} \sigma /(d p d \Omega)$ for incoming protons of $12.9 \mathrm{GeV} / \mathrm{c}$ on an aluminium target as a function of pion momentum $\mathrm{p}$ for pion polar angle $60 \leq \theta<90 \mathrm{mrad}$. The results of GEANT4 simulations with different hadronic models are compared to the HARP experimental data . . . . . . . . . . . . . . . . . . . . . . .

4.5 Measured double-differential $\pi^{+}$production cross-section $d^{2} \sigma /(d p d \Omega)$ for incoming protons of $12.9 \mathrm{GeV} / \mathrm{c}$ on an aluminium target as a function of pion momentum $\mathrm{p}$ for pion polar angle $90 \leq \theta<120 \mathrm{mrad}$. The results of GEANT4 simulations with different hadronic models are compared to the HARP experimental data . . . . . . . . . . . . . . . . . . . . .

4.6 Measured double-differential $\pi^{+}$production cross-section $d^{2} \sigma /(d p d \Omega)$ for incoming protons of $12.9 \mathrm{GeV} / \mathrm{c}$ on an aluminium target as a function of pion momentum $\mathrm{p}$ for pion polar angle $120 \leq \theta<150 \mathrm{mrad}$. The results of GEANT4 simulations with different hadronic models are compared to the HARP experimental data . . . . . . . . . . . . . . . . . . . . .

4.7 Measured double-differential $\pi^{+}$production cross-section $d^{2} \sigma /(d p d \Omega)$ for incoming protons of $12.9 \mathrm{GeV} / \mathrm{c}$ on an aluminium target as a function of pion momentum $\mathrm{p}$ for pion polar angle $150 \leq \theta<180 \mathrm{mrad}$. The results of GEANT4 simulations with different hadronic models are compared to the HARP experi-

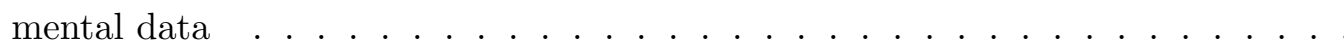

4.8 Measured double-differential $\pi^{+}$production cross-section $d^{2} \sigma /(d p d \Omega)$ for incoming protons of $12.9 \mathrm{GeV} / \mathrm{c}$ on an aluminium target as a function of pion momentum $\mathrm{p}$ for pion polar angle $180 \leq \theta<120 \mathrm{mrad}$. The results of GEANT4 simulations with different hadronic models are compared to the HARP experimental data . . . . . . . . . . . . . . . . . . . . . . .

4.9 Measured double-differential $\pi^{+}$production cross-section $d^{2} \sigma /(d p d \Omega)$ for incoming protons of $12.9 \mathrm{GeV} / \mathrm{c}$ on an aluminium target as a function of pion momentum $\mathrm{p}$ for pion polar angle $30 \leq \theta<210 \mathrm{mrad}$. The results of MARS simulations are compared to the HARP experimental data. The $\mathrm{X}$ and $\mathrm{Y}$ axes are $\mathrm{GeV} / \mathrm{c}$ and $(\mathrm{mb} /(\mathrm{GeV} / \mathrm{c} \mathrm{sr}))$ respectively . . . . . . . . . . . . . . . .

4.10 Percentage difference between the simulated results the HARP measured doubledifferential $\pi^{+}$production cross-section $d^{2} \sigma /(d p d \Omega)$ for incoming protons of 12.9 $\mathrm{GeV} / \mathrm{c}$ on an aluminium target. The GEANT4-QGSC (blue) and and MARS15( red) simulations are compared. Y-axis shows ((Simulated Result-HARP Result)/(HARP result) $) * 100$ and $\mathrm{X}$-axis shows total momentum of pion secondaries. Plots cover the whole 30-210 mrad angular range . . . . . . . . . . . .

4.11 Measured differential $\pi^{+}$production cross-section $d \sigma / d p$ for incoming protons of $12.9 \mathrm{GeV} / \mathrm{c}$ on an aluminium target. The results of of GEANT4 simulations with different hadronic models are compared to the HARP experimental data .

4.12 Measured differential $\pi^{+}$production cross-section $d \sigma / d p$ for incoming protons of $12.9 \mathrm{GeV} / \mathrm{c}$ on an aluminium target. The results of MARS simulations are compared to the HARP experimental data and GEANT4 QGSC model results . 
4.13 Measured differential $\pi^{+}$production cross-section $d \sigma / d \theta$ for incoming protons of $12.9 \mathrm{GeV} / \mathrm{c}$ on an aluminium target. The results of GEANT4 simulations with different hadronic models are compared to the HARP experimental data . . . .

4.14 Measured differential $\pi^{+}$production cross-section $d \sigma / d \theta$ for incoming protons of $12.9 \mathrm{GeV} / \mathrm{c}$ on an aluminium target. The results of MARS simulations are compared to the HARP experimental data and GEANT4 QGSC model results . 84

5.1 3D layout of the full MICE Stage-VI experiment [67] . . . . . . . . . . . 87

5.2 Planned staged evolution of the MICE experiment . . . . . . . . . . . . . 88

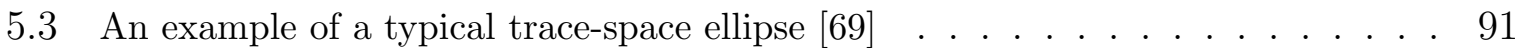

5.4 a) Resolution of $P_{T}$ as a function of $P_{T}$ b) Resolution of $P_{T}$ as a function of $P_{z}$ c) Resolution of $P_{z}$ as a function of $P_{T}$ d) Resolution of $P_{z}$ as a function of $P_{z}$ [80] 97

5.5 Scintillation light thresholds for both aerogel Cherenkov devices. The numbered regions indicate changes in triggering behaviour. Region I one unit triggers, region II both units trigger for muons only, region III both units trigger. . . . . 100

5.6 Engineering drawing of the RF cavity and absorber layout for the full MICE Stage VI. The eight RF cavities can be seen with curved terminating beryllium windows and coupling coils. The three absorber-focus-coil modules can clearly be seen. All superconducting magnetic elements are shown in red. . . . . . . . 101

6.1 Evolution of energy (blue line, units are $\mathrm{MeV}$ ) and momentum (red line, units are $\mathrm{MeV} / \mathrm{c}$ ) from injection to extraction . . . . . . . . . . . . . . . . 105

6.2 The ISIS neutron spallation source. Accelerator diagnostics are indicated. One should note the position of the old HEP beamline in the lower right of the diagram which is essentially the position of the MICE beamline. Superperiod 7 is the straight section immediately downstream of this [83] . . . . . . . . . . 107

6.3 Schematic representation of the MICE target drive assembly . . . . . . . . . 108

6.4 Target test setup . . . . . . . . . . . . . . . . . . . . . . 109

6.5 High gain dependency of the unshielded detector phototubes as a function of the control voltage applied (left panel). The electrical layout of the phototubes (right panel) . . . . . . . . . . . . . . . . . . . . . . 110

6.6 Representation of the unshielded detector showing PMT layout and housing. . . 111

6.7 Transverse particle density detected in the $40 \times 40 \mathrm{~cm}^{2}$ detector using the MARS target distribution, showing that it is essentially flat in the $40 \times 40 \mathrm{~cm}^{2}$. . . . . 114

6.8 Simulated momentum truth distribution recorded by the unshielded detectors $\left(40 \times 40 \mathrm{~cm}^{2}\right)$ for all incident particles tracked from MARS target yields, for $1 \times 10^{7}$ protons incident on target . . . . . . . . . . . . . . . . . . 115

6.9 Simulated momentum truth distribution recorded by the shielded detectors $(40 \times$ $40 \mathrm{~cm}^{2}$ ) for all incident particles tracked from MARS target yields, for $1 \times 10^{7}$ protons incident on target . . . . . . . . . . . . . . . . . . 116

6.10 Simulated momentum truth distribution recorded by the unshielded detectors $\left(40 \times 40 \mathrm{~cm}^{2}\right)$ for all incident particles tracked from GEANT4 target yields, for $1 \times 10^{7}$ protons incident on target . . . . . . . . . . . . . . . . . 117

6.11 Simulated momentum truth distribution recorded by shielded detectors $(40 \times$ $40 \mathrm{~cm}^{2}$ ) for all incident particles tracked from GEANT4 target yields, for $1 \times 10^{7}$ protons incident on target . . . . . . . . . . . . . . . 118

6.12 Parametrised neutron efficiency curve as a function of incident neutron energy . 118 
6.13 A Typical coincidence observed in raw data observed in both unshielded detectors. Each colour denotes the signal from an individual tube . . . . . . . . . . 122

6.14 Evolution of the target position for static and pulsed run conditions over the 10ms spill [89] . . . . . . . . . . . . . . . . . . . . . . . . 122

6.15 The complete data-set for all run conditions are plotted for the unshielded detector as a function of time during the pulse. The red histograms show coincident hits for runs when the target was dipped into the beam. The blue histograms are coincident hits for the runs where the target was held inside the beam.

6.16 The complete data-set for all run conditions are plotted for the shielded detector as a function of time during the pulse. The red histograms show coincident hits for runs when the target was dipped into the beam. The blue histograms are coincident hits for the runs where the target was held inside the beam. . . . . . 123

6.17 Relationship between number of coincident hits recorded in the unshielded detectors and the mean beam-loss in ISIS superperiod 7 monitors. . . . . . . . . 126

6.18 Releationship between number of coincident hits recorded in the shielded detectors and the mean beam-loss in ISIS superperiod 7 monitors. . . . . . . . . . 126

6.19 Estimation of the number of single particles into the MICE angular acceptance. The result is extrapolated from the data collected by the unshielded detector . . 127

6.20 Estimation of the number of single particles into the MICE angular acceptance. The result is extrapolated from the data collected by the shielded detector . . . 127

6.21 The total beam loss in the last $2 \mathrm{~ms}$ as a function of the mean target depth between 8-10ms . . . . . . . . . . . . . . . . . . . 131

6.22 Estimation of the number of recorded singles into the MICE angular acceptance as a function of the mean target depth between $8-10 \mathrm{~ms}$. The result is extrapolated from the linear fit to data collected by the unshielded detector and scaled to the MICE angular acceptance . . . . . . . . . . . . . . . . . . . . 132

7.1 The location of the MICE experiment and beamline in relation to ISIS . . . . . 134

7.2 Particle production histograms as a function of momentum showing meson and lepton production from MARS (left panel) and GEANT4 (right panel). . . . . 136

7.3 The $\pi^{+}$momentum distribution from a LAHET simulation of $800 \mathrm{MeV}$ protons on a titanium target . . . . . . . . . . . . . . . . . . . 136

7.4 Engineering drawing showing the upstream section of the beamline inside the ISIS vault. The first quadrupole triplet and dipole can be seen placed on its raised support. . . . . . . . . . . . . . . . . . . . . . . 139

7.5 Open GL visualisation of the pion decay channel:- Upstream the grey vacuum pipe is shown as is the Type IV quad triplet. Both dipoles are shown (red) with the PSI decay solenoid (yellow) and proton absorber (cyan) between them . . . 140

7.6 Relationship between the reference pion and muon lattice central momenta [96] . 141

7.7 Visualisation of the muon matching section. Upstream one can see the downstream end of the decay solenoid (yellow), the proton absorber (cyan), the second dipole (large red). Also shown in this picture is horizontal collimation (red), this is not in the baseline but is currently being studied as a modification. Both sets of quad triplets are shown (green and blue volumes) as is TOF0 (thin white volume in centre), CKOV1 (larger white volume in centre) and TOF1 (thin white volume further downstream) . . . . . . . . . . . . . . . . . . . . . . . 142 
7.8 Analysis of momentum distribution for pions (red) and muons (blue) sampled entering the upstream Cherenkov detector (left panel). Typical time-of-flight separation between pions (red) and muons (blue) (right panel). Results obtained

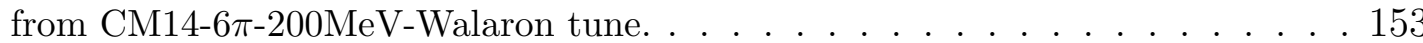

7.9 Analysis of idealised particle identification of pions (red) and muons (blue), achievable by the MICE beamline (left panel). Particle identification achievable by the beamline with estimation of the effect of TOF wall timing resolution (right panel). Results obtained from CM14-6 $\pi-200 \mathrm{MeV}$-Walaron tune . . . . . . 153

7.10 Results of GEANT4 implementation of the CM14- $6 \pi-200 \mathrm{MeV} / \mathrm{c}$ beamline optics tune. The plots show the $\mathrm{X}$ and $\mathrm{Y}(\mathrm{mm})$ spatial distributions of the beam at Time-of-flight detectors 0 and 1 and at the Cherenkov detectors. The dimensions of these detectors are TOF $0 \pm 250 \mathrm{~mm}$, TOF $1 \pm 240 \mathrm{~mm}$ and CKOV $\pm 200 \mathrm{~mm}$. . . 157

7.11 Ptot distribution at the entrance to the upstream MICE tracker for the CM14$6 \mathrm{pi}-200 \mathrm{MeV} / \mathrm{c}$ tune . . . . . . . . . . . . . . . . . . . 158

7.12 Results of GEANT4 implementation of the CM14- $6 \pi-200 \mathrm{MeV} / \mathrm{c}$ beamline optics tune. Plots show the evolution of the beam properties over the muon matching section. These properties are calculated for the reduced phase space volume with $215.31<E_{\mu}<252.2$ sampled at the first MICE tracker. Schematic representation of the beamline is indicated by different volumes on plots according to the following key: Matt red (horizontal focussing quad); Matt green (horizontal defocussing quad); Thin black (Iron shield); Thick black (tracker solenoid, only aperture correct); Blue (TOF0 and TOF1); Thick light blue (CKOV); Thin green (Diffuser) . . . . . . . . . . . . . . . . . . . . 160

7.13 Results of GEANT4 implementation of the CM14- $10 \pi-200 \mathrm{MeV} / \mathrm{c}$ beamline optics tune. The plots show the $\mathrm{X}$ and $\mathrm{Y}(\mathrm{mm})$ spatial distributions of the beam at Time-of-flight detectors 0 and 1 and at the Cherenkov detectors. The dimensions of these detectors are TOF0 $\pm 250 \mathrm{~mm}$, TOF $1 \pm 240 \mathrm{~mm}$ and CKOV $\pm 200 \mathrm{~mm}$. . . 161

7.14 Ptot distribution at the entrance to the upstream MICE tracker for the CM14$10 \mathrm{pi}-200 \mathrm{MeV} / \mathrm{c}$ tune . . . . . . . . . . . . . . . . . 162

7.15 Results of GEANT4 implementation of the CM14- $10 \pi-200 \mathrm{MeV} / \mathrm{c}$ beamline optics tune. Plots show the evolution of the beam properties over the muon matching section. These properties are calculated for the reduced phase space volume with $215.31<E_{\mu}<252.2$ sampled at the first MICE tracker. Schematic representation of the beamline is indicated by different volumes on plots according to the following key: Matt red (horizontal focussing quad); Matt green (horizontal defocussing quad); Thin black (Iron shield); Thick black (tracker solenoid, only aperture correct); Blue (TOF0 and TOF1); Thick light blue (CKOV); Thin green (Diffuser) . . . . . . . . . . . . . . . . . . . 163

7.16 Results of GEANT4 implementation of the CM14- $1 \pi-200 \mathrm{MeV} / \mathrm{c}$ beamline optics tune. The plots show the $\mathrm{X}$ and $\mathrm{Y}(\mathrm{mm})$ spatial distributions of the beam at Time-of-flight detectors 0 and 1 and at the Cherenkov detectors. The dimensions of these detectors are TOF $0 \pm 250 \mathrm{~mm}, \mathrm{TOF} 1 \pm 240 \mathrm{~mm}$ and CKOV $\pm 200 \mathrm{~mm}$. . . 164

7.17 Ptot distribution at the entrance to the upstream MICE tracker for the CM14$1 \mathrm{pi}-200 \mathrm{MeV} / \mathrm{c}$ tune . . . . . . . . . . . . . . 166 
7.18 Results of GEANT4 implementation of the CM14- $1 \pi-200 \mathrm{MeV} / \mathrm{c}$ beamline optics tune. Plots show the evolution of the beam properties over the muon matching section. These properties are calculated for the reduced phase space volume with $215.31<E_{\mu}<252.2$ sampled at the first MICE tracker. Schematic representation of the beamline is indicated by different volumes on plots according to the following key: Matt red (horizontal focussing quad); Matt green (horizontal defocussing quad); Thin black (Iron shield); Thick black (tracker solenoid, only aperture correct); Blue (TOF0 and TOF1); Thick light blue (CKOV); Brown (Collimator) . . . . . . . . . . . . . . 167

7.19 Cooling performance of a respresentative $6.25 \pi \mathrm{mm} \mathrm{mrad,} 200 \mathrm{MeV} / \mathrm{c}$ muon beam through MICE. The four-dimensional transverse emittance of the beam is shown as a function of length along the channel given in the MICE coordinate system where zero is defined to the the centre of the middle absorber. . . . . . . 168 


\section{List of Tables}

1.1 Calculated neutrino flux from the salt phase of the SNO detector . . . . . . . 18

2.1 Oscillation channels for a storage ring populated by negative muons. The charge conjugate oscillations are present when positive muons are also stored in the ring 35

3.1 Details on envisaged and current proton drivers [48] . . . . . . . . . . . . . . . 45

3.2 Incident proton energies used in simulations [56] . . . . . . . . . . . . . . 49

3.3 GEANT 4 Hadronic Physics Summary . . . . . . . . . . . . . . . . . . 53

4.1 Number of events for different HARP data sets with $5 \% \lambda_{I}$ aluminium target and without. For details of trigger selection criteria see [63] . . . . . . . . . 69

4.2 Geant4 simulation parameters . . . . . . . . . . . . . . . 70

4.3 Results from the HARP experiment for the double-differential cross-section in the laboratory system $\left(d^{2} \sigma^{\pi_{A R P}^{+}} / d p d \Omega\right)$. The values shown were calculated using the official HARP analysis discussed in [63] . . . . . . . . . . . . 73

6.1 Data acquisition summary . . . . . . . . . . . . . . . . 113

6.2 Simulation parameters . . . . . . . . . . . . . . . . . 113

6.3 Un-normalised rates for both shielded and unshielded detectors $\left(40 \times 40 \mathrm{~cm}^{2}\right)$ using MARS derived target yields. Also shown is the difference between results as a percentage of the unshielded rate for $1 \times 10^{7}$ protons incident on target. Statistics are very low for meson and leptons and hence the $\%$ difference yields no real result, they are included for completeness . . . . . . . . . . . . . . . . 114

6.4 Un-normalised rates for both shielded and unshielded detectors $\left(40 \times 40 \mathrm{~cm}^{2}\right)$ using GEANT4 derived target yields for $1 \times 10^{7}$ protons incident on target. Also shown is the difference between results as a percentage of the unshielded rate. Statistics are very low for meson and leptons and hence the $\%$ difference yields no real result, they are included for completeness . . . . . . . . . . . . . . 115

6.5 Un-normalised rates at upstream face of both scintillators for shielded and unshielded detectors from the MARS target yield. This gives an estimate of the efficiency of detecting a coincidence in both phototubes . . . . . . . . . . . . . 119

6.6 Un-normalised rates at upstream face of both scintillators for shielded and unshielded detectors from the GEANT4 target yield. This gives an estimate of the efficiency of detecting a coincidence in both phototubes . . . . . . . . . . . . . 119

6.7 Summary of data runs and target operating conditions . . . . . . . . . . . . . 120

6.8 Accumulated coincident PMT hit statistics between $8-10 \mathrm{~ms}$. . . . . . . . . . . 125

6.9 Mean data between 8-10ms for both shielded and unshielded detectors . . . . . 125 
6.10 Results of linear least square fits to the number of particles detected in both the shielded and unshielded detectors as a function of total beam loss between 8-10ms. Also detailed are the results from similar fits extrapolating the detector results to the entire MICE beamline angular acceptance. (S) denotes value calculated for the shielded detector and (US) for the unshielded detector . . . . 125

6.11 The number of protons lost from the ISIS beam as an absolute number and as a fraction of the total number of protons in each bunch. The values calculated correspond to the number of protons on target for the range of dipped target settings. . . . . . . . . . . . . . . . . 129

6.12 The calculated number of non-neutron singles into the $1 \mathrm{~cm}^{2}$ detector acceptance using MARS, GEANT4 and data. Results are shown for both shielded and unshielded detector cases. . . . . . . . . . . . . . . . . . . . . . 130

6.13 Mean target depth and mean total beam loss between 8-10ms . . . . . . . 130

7.1 Detailed simulation parameters in modeling the MICE target and determining normalisations to estimate tertiary beam rates . . . . . . . . . . . 137

7.2 Normalisation parameters for $200 \mathrm{MeV} / \mathrm{c}$ beams over a range of emittances . . . 138

7.3 Beamline magnets physical properties . . . . . . . . . . . . . . . . 144

7.4 Summary of study of momentum drop and multiple scattering analysis in the MICE beamline. . . . . . . . . . . . . . . . . . . . . . . . . 148

7.5 Summary of material content of beamline introduced by particle identification detectors. . . . . . . . . . . . . . . . . . . 152

7.6 Original single unit CKOV implementation. . . . . . . . . . . . . . . . . 152

7.7 Summary of non-detector material content of beamline, for radiation length calculation the diffuser for the $7.1 \pi \mathrm{mm}$ mrad beamline is assumed . . . . . . . . 154

7.8 Beamline optics solutions for $200 \mathrm{MeV} / \mathrm{c}$ MICE beamline. Dipole and solenoidal

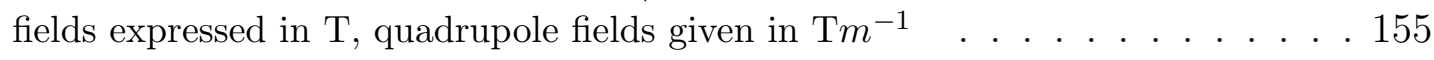

7.9 Purity of $6 \pi, 200 \mathrm{MeV} / \mathrm{c}$ beamline sampled at each of the beamline detectors and upstream tracker . . . . . . . . . . . . . . . . . 156

7.10 Positive muon rates in $\mathrm{MHz}$ along the mid-emittance $200 \mathrm{MeV} / \mathrm{c}$ MICE beamline 156

7.11 Purity of $10 \pi, 200 \mathrm{MeV} / \mathrm{c}$ beamline sampled at each of the beamline detectors and upstream tracker . . . . . . . . . . . . . . . . . . . . . 162

7.12 Positive muon rates in $\mathrm{MHz}$ along the large emittance $200 \mathrm{MeV} / \mathrm{c}$ MICE beamline162

7.13 Positive muon rates in $\mathrm{MHz}$ along the low emittance $200 \mathrm{MeV} / \mathrm{c}$ MICE beamline 165

7.14 Purity of $1 \pi, 200 \mathrm{MeV} / \mathrm{c}$ beamline sampled at each of the beamline detectors and upstream tracker . . . . . . . . . . . . . . . . . 165 


\section{Chapter 1}

\section{Neutrino Physics}

\subsection{Discovery of the neutrino}

The neutrino was effectively born by proxy on the 4th December 1930 in a letter by the famous physicist Wolfgang Pauli. Addressing the meeting in Tubingen which he could not attend in person preferring instead a ball in Zurich, the letter read [1]:

Dear Radioactive Ladies and Gentlemen,

As the bearer of these lines, to whom I graciously ask you to listen, will explain to you more exactly, considering the "false" statistics of the $N$ and 6Li nuclei, as well as the continuous beta spectrum, I have hit upon a desperate remedy to save the "exchange theorem" of statistics and the theorem of conservation of energy. Namely, the possibility that there could exist in the nuclei electrically neutral particles, that I wish to call neutrons, which have spin 1/2 and obey the exclusion principle and which further differ from light quanta in that they do not travel with the velocity of light. The mass of the neutrons should be of the same order of magnitude as the electron mass and in any event not larger than 0.01 proton masses. The continuous beta spectrum would then become understandable by the assumption that in beta decay a neutron is emitted in addition to the electron such that the sum of the energies of the neutron and the electron is constant

Now, the next question is what forces act upon the neutrons. The most likely model for the 
neutron seems to me to be, on wave mechanical grounds (more details are known by the bearer of these lines), that the neutron at rest is a magnetic dipole of a certain moment $\mu$. Experiment probably required that the ionising effect of such a neutron should not be larger than that of a $\gamma$ ray, and thus $\mu$ should probably not be larger than $10^{-13} \mathrm{~cm}$.

But I don't feel secure enough to publish anything about this idea, so I first turn confidentially to you, dear radioactives, with a question as to the situation concerning experimental proof of such a neutron, if it has something like about 10 times the penetrating capacity of a $\gamma$ ray.

I agree that my remedy could seem incredible because one should have seen those neutrons very earlier if they really exist. But only the one who dares can win and the difficult situation, due to the continuous structure of the beta spectrum, is lighted by a remark of my honored predecessor, Mr Debye, who told me recently in Bruxelles: "Oh, It's well better not to think to this at all, like new taxes". From now on, every solution to the issue must be discussed. Thus, dear radioactive people, look and judge. Unfortunately, I cannot appear in Tubingen personally since I am indispensable here in Zurich because of a ball on the night of $6 / 7$ December. With my best regards to you, and also to Mr Back.

Your humble servant

W. Pauli

This letter was a drastic response to one of the hottest topics of the time. It had been known for many years that the spectrum of $\beta$ particles emitted from the radioactive decay of nuclei was continuous. The plot in Figure 1.1 shows the continuous beta spectrum of this contemporary study of beta emission in Radium E (Bismuth-210). As Pauli briefly mentions this puzzling behavior suggests violation of energy conservation as two-body decay does not allow for a continuous spectrum. The "neutron" Pauli describes here is a solution to this problem without demanding the violation of conservation of energy by requiring that there exists another particle that participates in the decay but is not directly detected. It is now understood that the "neutron" Pauli described has a mass greater than that of the proton and is too heavy to be the bold beta spectrum explanation first proposed.

The continuous electron energy spectrum was explained in 1934 by another famous physicist 


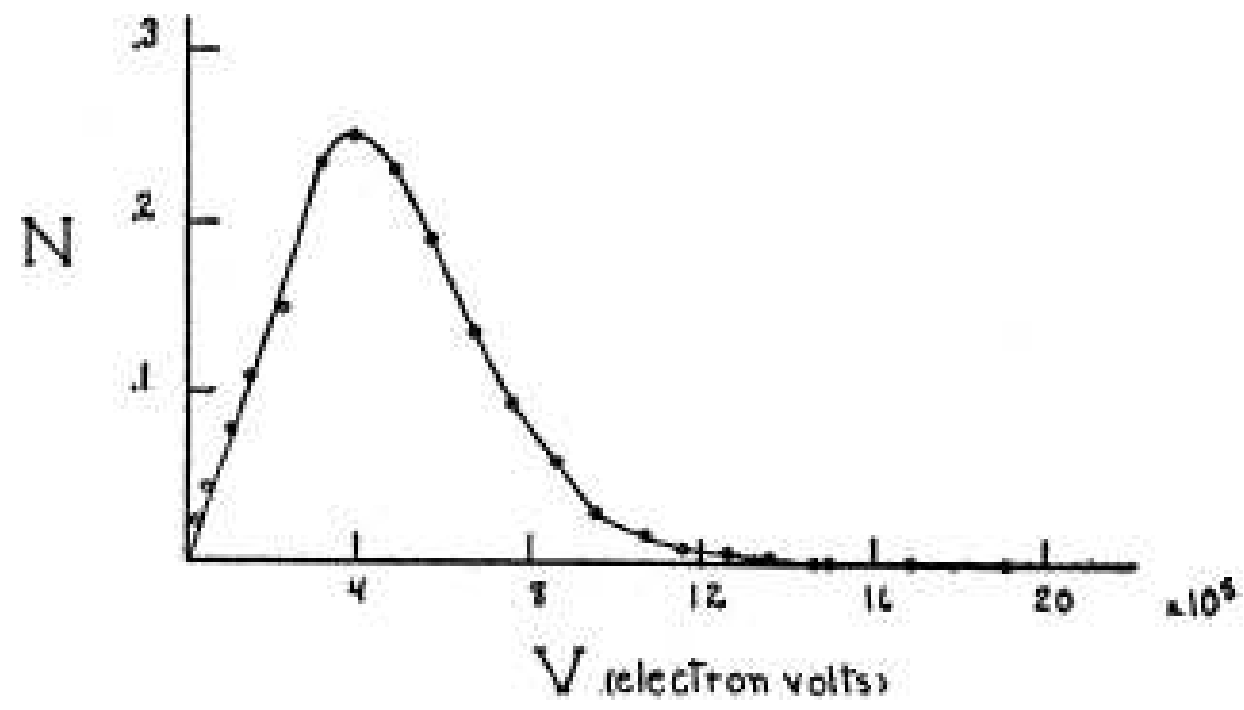

Figure 1.1: Energy distribution curve for the beta spectrum of Radium E [2]

Enrico Fermi. He realised that the $\beta$ decay was due to the decay of the neutron to a proton, an electron and another particle whose existence was, although slightly inadequately, postulated by Pauli in his letter to Tubingen. When asked if this particle was that described by Pauli, Fermi replied that yes it was only smaller and he called it the neutrino (the Italian translation being "little neutral one"). This reaction occurred via the newly discovered weak interaction and Fermi was able to deduce the strength of the associated coupling constant $G_{F}$.

The discovery of the neutrino did not come for a little more than two decades later in 1956 . Fred Reines and Clyde Cowan discovered the first electron (anti)neutrino published from results from their experiment at the Savannah River nuclear reactor [3]. Reines won the Nobel prize for this discovery.

\subsection{Neutrino Oscillation Theory}

\subsubsection{Three flavour oscillation in vacuum}

The neutrino is, as Pauli hinted to, extremely difficult to detect and therefore study. The reason being that having no electric charge and being virtually massless the only viable avenue of investigation is by studying evidence of their weak interactions. The neutrino participates in both neutral and charged current interactions. 
Neutrinos are known to have three weakly interacting flavours the electon(e), muon $(\mu)$ and $\operatorname{tau}(\tau)$ neutrinos. The evidence for having three active flavour eigenstates comes from neutral $Z_{0}$ production in electron-positron collisions at LEP. From the measurement of the invisible decay width of the $Z_{0}$ resonance the number of light (i.e. $m_{\nu}<m_{Z} / 2$ ) neutrino flavours is $N_{\nu}=2.9841 \pm 0.0083[4]$

The flavour state of the neutrino produced in weak interactions of the form $W^{ \pm} \rightarrow l_{\alpha}^{ \pm}+\nu_{\alpha}\left(\bar{\nu}_{\alpha}\right)$ can be expressed as a sum of the mass eigenstates as shown in (1.1)

$$
\left|\nu_{\alpha}\right\rangle=\sum_{i} U_{\alpha i}^{*}\left|\nu_{i}\right\rangle
$$

where $\mathrm{U}$ is a unitary mixing matrix of the form:

$$
\mathrm{U}=\left(\begin{array}{ccc}
c_{13} c_{12} & c_{13} s_{12} & s_{13} e^{-i \delta} \\
-c_{23} s_{12}-s_{13} s_{23} c_{12} e^{i \delta} & c_{23} c_{12}-s_{13} s_{23} s_{12} e^{i \delta} & c_{13} s_{23} \\
s_{23} s_{12}-s_{13} c_{23} c_{12} e^{i \delta} & -s_{23} c_{12}-s_{13} c_{23} s_{12} e^{i \delta} & c_{13} c_{23}
\end{array}\right)
$$

and e.g. $s_{23}=\sin \left(\theta_{23}\right)$ and $c_{13}=\cos \left(\theta_{13}\right)$ etc and $\delta$ is the CP violating phase.

The mixing matrix for neutrino oscillations involving all three weak neutrino eigenstates $\nu_{\alpha=e, \mu, \tau}$ can be expressed as shown in Figure 1.2. The mixing matrix performs a mapping for a basis change in 3D flavour space. The oscillation causes a rotation around each axis such that the orthogonal mass basis vectors and orthogonal flavour basis vectors are no longer perpendicular. Data from atmospheric and solar neutrino studies that will be detailed later show the pattern for the masses of the three weakly coupled mass eigenstates can be described as in Figure 1.3. The absolute value for the masses is not well known, only the mass-squared differences of which there are two. These are generally referred to as the "normal" and "inverted" hierarchies depending on whether the smaller (atmospheric) mass-squared difference describes flavour oscillation between the two heaviest or two lightest mass eigenstates.

The probability amplitude for neutrino oscillation from flavour $\nu_{\alpha} \rightarrow \nu_{\beta}$ can be expressed in the following form:

$$
\begin{aligned}
\operatorname{Amp}\left[\nu_{\alpha} \rightarrow \nu_{\beta}\right] & =\sum_{i}\left(\operatorname{Amp}\left[\nu_{i} \text { produced from } l_{\alpha}\right]\right. \\
& \left.\times \operatorname{Amp}\left[\nu_{i} \text { propagates }\right] \times \operatorname{Amp}\left[\nu_{i} \text { produced from } l_{\beta}\right]\right)
\end{aligned}
$$




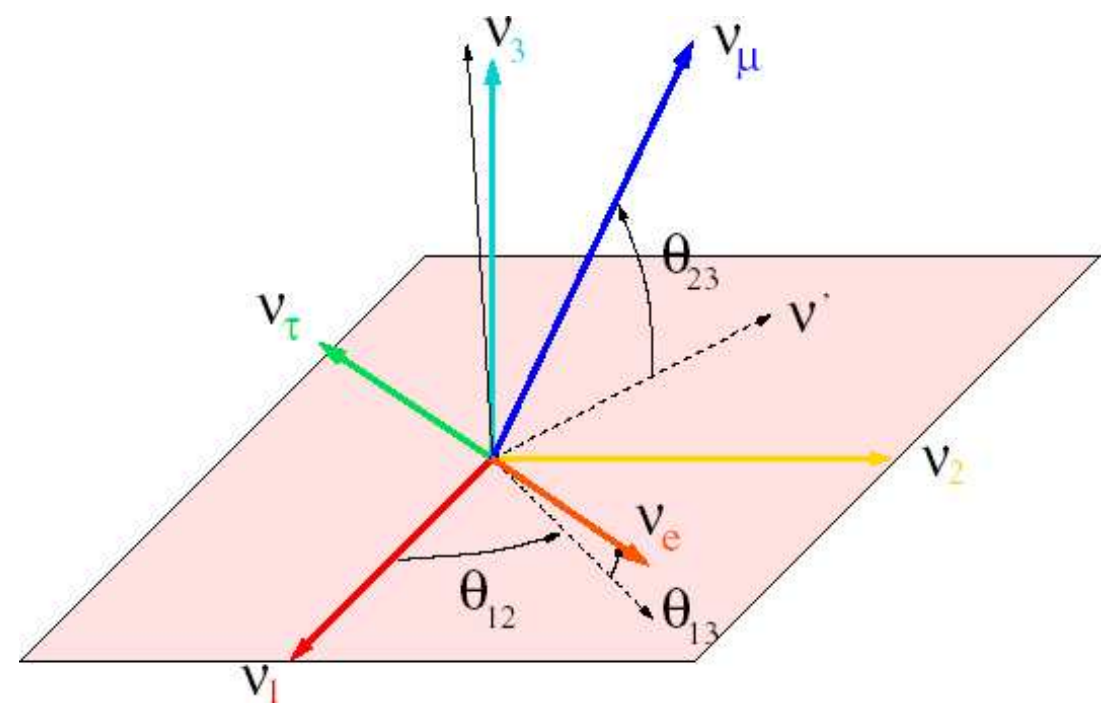

Figure 1.2: Oscillation induced 3D rotation of flavour basis with respect to the mass basis [5]

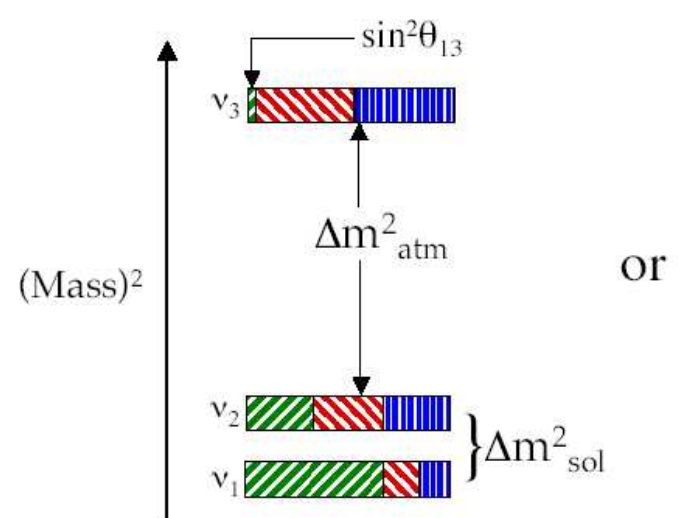

Normal

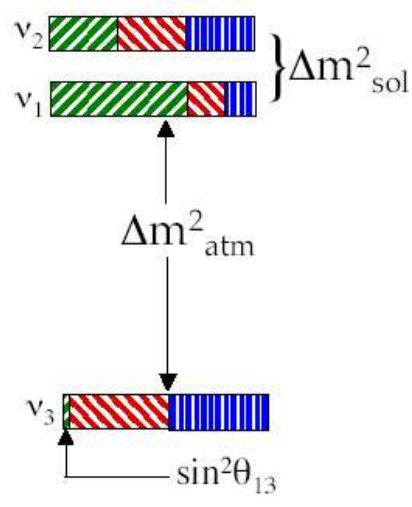

Inverted

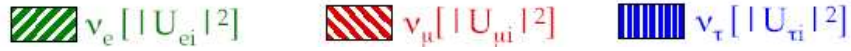

Figure 1.3: The possible neutrino mass-hierarchies assuming no sterile neutrinos. Also shown are approximate flavour content of each mass eigenstate [6]

To determine the middle term of RHS of (1.2) one uses the time-dependent Schrödinger eqn. in the neutrino rest frame:

$$
i \frac{\partial}{\partial \tau_{i}}\left|\nu_{i}\left(\tau_{i}\right)\right\rangle=H\left|\nu_{i}\left(\tau_{i}\right)\right\rangle=m_{i}\left|\nu_{i}\left(\tau_{i}\right)\right\rangle
$$

which yields a mass eigenstate expression for the neutrino after a period $\tau_{i}$ of 


$$
\left|\nu_{i}\left(\tau_{i}\right)\right\rangle=e^{-i m_{i} \tau_{i}}\left|\nu_{i}\left(\tau_{i}=0\right)\right\rangle
$$

Furthermore the amplitude that the neutrino propagates for a time $\tau_{i}$ is equal to the amplitude of finding the neutrino in the same state vector as it was when it was created i.e. $\left\langle\nu_{i}(0)|| \nu_{i}\left(\tau_{i}\right)\right\rangle$. This solution is in the mass eigenstate rest frame. One would like, for phenomenological reasons, to express this in the laboratory frame.

Thus for a neutrino in the laboratory rest frame the mass state will evolve in time $\mathrm{t}$ and distance L (measured in lab frame) with the phase factor:

$$
e^{-i m_{i} \tau_{i}}=e^{-i\left(E_{i} t-p_{i} L\right)}=e^{-i\left(E_{i}-p_{i}\right) L}
$$

This expression assumes Lorentz invariance and that the neutrino is highly relativistic such that $\mathrm{c}=1$ and $m_{i} \tau_{i}=E_{i} t-p_{i} L \cong\left(E_{i}-p_{i}\right) L$. Neutrino oscillations are only important when the mass eigenstates are coherent and this is true for neutrino states that share the same energy (E). Using $p_{i}=\sqrt{E^{2}-m_{i}^{2}}$ the neutrino propagator is modified readily. The amplitude for neutrino vacuum oscillation after travelling a distance $\mathrm{L}$ is given by (1.6):

$$
\operatorname{Amp}\left[\nu_{i} \text { propagation }\right]=e^{-i m_{i}^{2} \frac{L}{2 E}}
$$

Thus a neutrino created by a weak interaction as flavour $\nu_{\alpha}$ can be expressed at a distance L by a combination of weak flavour eigenstates $\nu_{\beta}$.

$$
\left|\nu_{\alpha}(L)\right\rangle \approx \sum_{i} U_{\alpha i}^{*} e^{-i\left(m_{i}^{2} / 2 E\right) L}\left|\nu_{i}\right\rangle \approx \sum_{\beta}\left(\sum_{i} U_{\alpha i}^{*} e^{-i\left(m_{i}^{2} / 2 E\right) L} U_{\beta i}\right)\left|\nu_{\beta}\right\rangle
$$

The probability of neutrino oscillation from flavour $\nu_{\alpha} \rightarrow \nu_{\beta}$ is equal to the $\left|\operatorname{Amp}\left(\nu_{\alpha} \rightarrow \nu_{\beta}\right)\right|^{2}$ and this yields the general form of neutrino oscillations in vacuum shown in (1.8).

$$
\begin{array}{r}
P\left(\nu_{\alpha} \rightarrow \nu_{\beta}\right)=\delta_{\alpha \beta}- \\
-\sum_{i>j} \Re\left(U_{\alpha i}^{*} U_{\beta i} U_{\alpha j} U_{\beta j}^{*}\right) \sin ^{2}\left(\triangle_{i j}^{2} \frac{L}{4 E}\right) \\
+2 \sum_{i>j} \Im\left(U_{\alpha i}^{*} U_{\beta i} U_{\alpha j} U_{\beta j}^{*}\right) \sin \left(\triangle_{i j}^{2} \frac{L}{4 E}\right)
\end{array}
$$


For illustrative purposes it is useful to show the two flavour oscillation expression for the probability of oscillation from $\nu_{e} \rightarrow \nu_{\mu}$. In this reduced flavour space the mixing matrix is of the form:

$$
\mathrm{U}=\left(\begin{array}{cc}
\cos \theta & \sin \theta \\
-\sin \theta & \cos \theta
\end{array}\right)
$$

The amplitude of the oscillation is given by:

$$
\begin{aligned}
\operatorname{Amp}\left(\nu_{e} \rightarrow \nu_{\mu}\right) & =\sum_{i=1,2} U_{\mu 1}^{*} U_{e i} e^{-i m_{i}^{2} L / 2 E} \\
& =-\sin \theta \cos \theta e^{-i m_{i}^{2} L / 2 E}+\sin \theta \cos \theta e^{-i m_{2}^{2} L / 2 E}
\end{aligned}
$$

The probability is given as previously stated as the modulus of the square of the probability amplitude and is given by the following expression:

$$
\begin{aligned}
P\left(\nu_{e} \rightarrow \nu_{\mu}\right) & =\sin ^{2} 2 \theta\left|\frac{e^{i \Delta m L / 4 E}-e^{i \Delta m L / 4 E}}{2 i}\right| \\
& =\sin ^{2} 2 \theta \sin ^{2}\left(\triangle m^{2} \frac{L}{4 E}\right)
\end{aligned}
$$

\subsubsection{Neutrino Oscillations in matter}

The previous discussion has centred around neutrino behaviour in vacuum. The oscillatory behaviour of the neutrinos are affected when the propagation is in a matter medium. This is known as the MSW (Mikheyev, Smirnov, Wolfenstein) effect after seminal contributions from those scientists on this subject [7], [8].

The neutrino has both coherent forward scattering charged-current and neutral current interactions with electrons and quarks. The Feynman diagrams of these processes can be seen in Figure 1.4. One can observe from these diagrams that while all neutrino flavour eigenstates couple to electrons found in the propagation medium via charged current interactions only the $\nu_{e}$ component couples via a charged current processes. It follows from this that the interaction energy in these interactions can modify the Hamiltonian governing neutrino propagation.

The interaction potential for both neutral and charged current interactions is given by $(1.9),(1.10)$.

$$
V_{w}=+\sqrt{2} G_{F} N_{e} \equiv A
$$



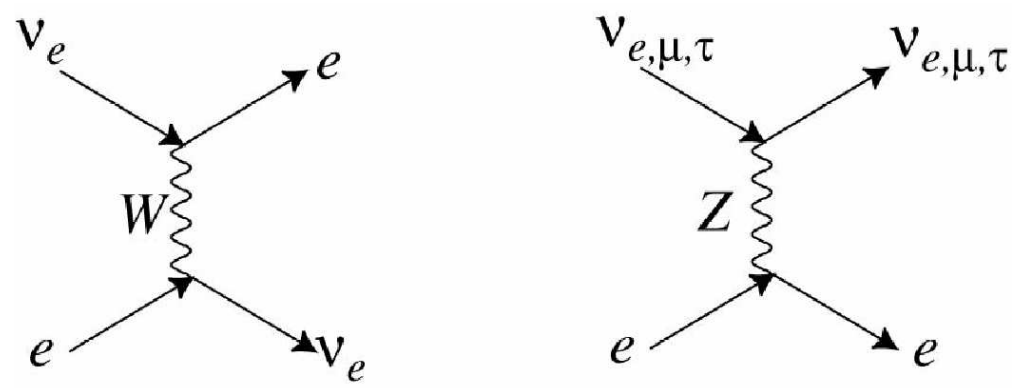

Figure 1.4: Neutral-current and charged-current neutrino-electron scattering diagrams

$$
V_{z}=-\frac{\sqrt{2}}{2} G_{F} N_{n}
$$

where $G_{F}$ is the Fermi constant and $N_{e}$ and $N_{n}$ are the electron and neutron number densities in the propagation matter and $\mathrm{A}$ is "matter" parameter often mentioned in the literature. To account for this additional interaction energy it is customary to construct an effective Schrödinger equation with a modified Hamiltonian.

If one deals with two neutrino $\nu_{e} \rightarrow \nu_{\mu}$ oscillation the modified Hamiltonian is given by the following:

$H_{M}=H_{v a c}+V_{w}\left[\begin{array}{ll}1 & 0 \\ 0 & 0\end{array}\right]+V_{z}\left[\begin{array}{ll}1 & 0 \\ 0 & 1\end{array}\right]=H_{v a c}+\frac{V_{w}}{2}\left[\begin{array}{cc}1 & 0 \\ 0 & -1\end{array}\right]+\frac{V_{w}}{2}\left[\begin{array}{cc}1 & 0 \\ 0 & 1\end{array}\right]+\frac{V_{z}}{2}\left[\begin{array}{ll}1 & 0 \\ 0 & 1\end{array}\right]$

Note that for neutrino oscillations only relative phases are important. This leads to the discarding of the last two terms in the RHS of the equations to yield:

$$
\begin{gathered}
H_{M}=\frac{\Delta m^{2}}{4 E}\left[\begin{array}{cc}
-(\cos 2 \theta-x) & \sin 2 \theta \\
\sin 2 \theta & \cos 2 \theta-x
\end{array}\right] \\
\text { with } x \equiv \frac{V_{W} / 2}{\Delta m^{2} / 4 E}=\frac{2 \sqrt{2} G_{F} N_{e} E}{\Delta m^{2}}
\end{gathered}
$$

If one then defines:

$$
\Delta m_{M}^{2} \equiv \Delta m^{2} \sqrt{\sin ^{2} 2 \theta+(\cos 2 \theta-x)^{2}}
$$

and, 


$$
\sin ^{2} 2 \theta_{M} \equiv \frac{\sin ^{2} 2 \theta}{\sin ^{2} 2 \theta+(\cos 2 \theta-x)^{2}}
$$

this equation is then of the same form as the equation governing vacuum oscillation under the transformation $\left(\Delta m^{2}, \theta\right) \rightarrow\left(\Delta m_{M}^{2}, \theta_{M}\right)$ i.e.:

$$
H_{M}=\frac{\Delta m_{M}^{2}}{4 E}\left[\begin{array}{cc}
\cos 2 \theta_{M} & \sin 2 \theta_{M} \\
\sin 2 \theta_{M} & \cos 2 \theta_{M}
\end{array}\right]
$$

Assuming that our neutrino does not travel very deep in the earth, the matter density along the propagation can be thought to be roughly constant. This implies that the Hamiltonian $\left(H_{M}\right)$ is position independent, which is also the case for the vacuum Hamiltonian. Under this assumption the probability for flavour change under the influence of matter propagation is given by equation (1.16), which again is the same form as the vacuum probability under the transformation $\left(\Delta m^{2}, \theta\right) \rightarrow\left(\Delta m_{M}^{2}, \theta_{M}\right)$

$$
P_{M}\left(\nu_{e} \rightarrow \nu_{\mu}\right)=\sin ^{2} 2 \theta_{M} \sin ^{2}\left(\triangle m_{M}^{2} \frac{L}{4 E}\right)
$$

As a consequence of the modification of the Hamiltonian due to the charged-current potential being different for $\nu$ and $\bar{\nu}$ the sign of the variable $x$ differs for neutrino and anti-neutrino. It follows that the mass squared difference and mixing angle given in Equations 1.13, 1.14 are modified with $x$ having either a positive $(\nu)$ or negative $(\bar{\nu})$ sign. It can also be noted that the $\triangle m^{2}$ in the denominator of $x$ also introduces the possibility of a sign change. These details mean that the MSW matter effect causes an asymmetry between $\nu$ and $\bar{\nu}$. This effect can be used to determine whether the mass hierarchy is normal or inverted.

\section{$1.3 \quad$ Neutrino Phenomenology}

The discovery that neutrinos have mass and mix has led to an extremely active program of experiments focusing on the determination of key physical parameters which describe the behaviour of neutrinos. The three neutrino mixing angles $\theta_{12}, \theta_{23}, \theta_{13}$ must be determined to high precision as must the two known mass-squared differences between mass eigenstates $\Delta m_{21}^{2}, \Delta m_{31}^{2}$. Other important questions are: 
- What is the absolute mass scale of neutrino masses?

- How small is $\theta_{13}$ ?

- If $\theta_{13} \neq 0$ what is the value of the CP violating phase $\delta$ ?

- Is $\theta_{23}$ maximal?

- What is the neutrino mass hierarchy? Normal or inverted?

- Are neutrinos Dirac (distinct $\nu$ and $\bar{\nu}$ ) or Majorana (the $\nu$ and $\bar{\nu}$ are the same particle) fermions?

Answers to these questions also have many cosmological consequences. The small but finite mass of neutrinos contribute to the total energy in the universe at least as much as all the stellar matter combined. Also, the neutrinos and photons produced in the Big Bang outnumber the constituents of normal matter $\left(e^{-}, p, n\right)$ by a factor of $10^{7}$. Both these details highlight that precise neutrino parameter measurements are fundamental to our understanding of the universe.

\subsection{Atmospheric Neutrino Experiments}

A large number of the neutrinos detectable in terrestrial neutrino experiments are produced in the atmosphere. Cosmic rays incident on the atmosphere produce many secondaries. These are primarily pions which subsequently decay to muons in the reaction $\pi^{ \pm} \rightarrow \mu^{ \pm}+\nu_{\mu}\left(\bar{\nu}_{\mu}\right)$ with the muon then decaying to an electron or positron $\mu^{ \pm} \rightarrow e^{ \pm}+\left(\bar{\nu}_{\mu}\right) \nu_{\mu}+\left(\nu_{e}\right) \bar{\nu}_{e}$. The energy spectrum of cosmic ray produced neutrinos in this discussion ranges from $100 \mathrm{MeV} \rightarrow$ few $\mathrm{GeV}$.

The most important detection scheme used to study these neutrinos are water Cherenkov detectors with the key experiment the Super-Kamiokande (Super-K) detector [9]. The SuperKamiokande detector is a huge 50kton cylindrical detector housed at a depth of 2500 metreswater-equivalent in Gifu Prefecture, Japan. The neutrinos are detected by observing the leptons they produce in the water via the interaction $\nu+N \rightarrow l+X$ with the events denoted as being "fully contained" or "partially contained" depending on whether all the energy of the event is deposited inside the Super-K inner detector. The leptons produced travel faster than the speed of light in the medium and therefor emit Cherenkov light which is detected by 11200 photomultiplier-tubes. This detection technique is important as it enables tagging of the flavour of 
the lepton and hence neutrino that produced it and also the direction of the neutrino. The directional information was important at verifying the direction of the neutrinos perceived to originate from the sun and this is discussed in the solar neutrino section. The directional information is important in terms of atmospheric neutrinos because the direction gives the oscillation distance. This allows one to study neutrino flux as a function of zenith angle (oscillation distance). Neutrinos incident downwards on the detector $(\cos \theta=1)$ travel approximately $15 \mathrm{~km}$ before entering the detector, whereas neutrinos incident upwards on the detector $(\cos \theta=-1)$ travel through the Earth for roughly $13000 \mathrm{~km}$.

A zenith angle analysis of $\nu_{e}$ and $\nu_{\mu}$ fluxes using Super-K data can be seen in Figure 1.5. It is observed that the flux of $\nu_{\mu}$ that have passed through the earth is roughly half of the flux determined to be coming from directly overhead. The $\nu_{e}$ flux distribution does not show a similar trend. This result is understood to come from an effective $\nu_{\mu} \rightarrow \nu_{\tau}$ oscillation and was determined to be the first conclusive proof of neutrino oscillation [10].
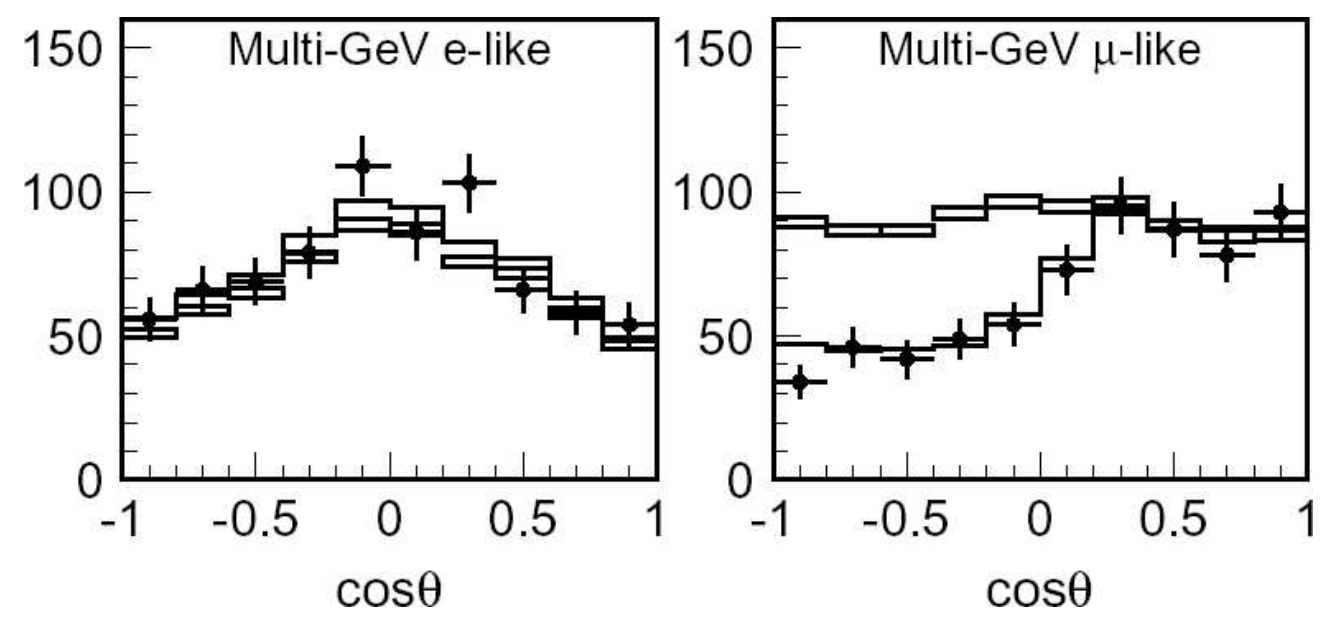

Figure 1.5: Super-Kamiokande zenith angle distributions for fully-contained 1-ring events. The points show data and the box histograms show Monte Carlo events assuming no oscillations. The solid line shows the best-fit expectation for $\nu_{\mu} \longleftrightarrow \nu_{\tau}$ oscillations with $\sin ^{2} 2 \theta=1.00$ and $\Delta m^{2}=$ $2.1 \times 10^{-3} \mathrm{eV}^{2}[10]$

The Super-K data also underwent an L/E analysis. The ratio of data over non-oscillated Monte Carlo as a function of L/E from this analysis is shown in Figure 1.7. It can be seen that the data agrees well with a best-fit 2 flavour $\nu_{\mu} \longleftrightarrow \nu_{\tau}$ oscillation. Two additional models for neutrino disapearance are plotted to check the statistical significance of this result. Analysis of these alternative models show that neutrino decay and the decoherence model are disfavoured 
by $4.8 \sigma$ and $5.3 \sigma$ respectively as they fail to reproduce the dip in the $\mathrm{L} / \mathrm{E}$ distribution. This result is the first direct evidence that the $\mathrm{L} / \mathrm{E}$ distribution of the neutrino survival probability follows the predicted oscillatory behaviour. The best-fit atmospheric neutrino parameters to fit this data are shown in Figure 1.6 and are at the 90\% C.L [11]:

$$
1.9 \times 10^{-3} e V^{2}<\Delta m_{a t m}^{2}=3.0 \times 10^{-3} \mathrm{eV}^{2} \sin ^{2} 2 \theta_{23}<0.9
$$

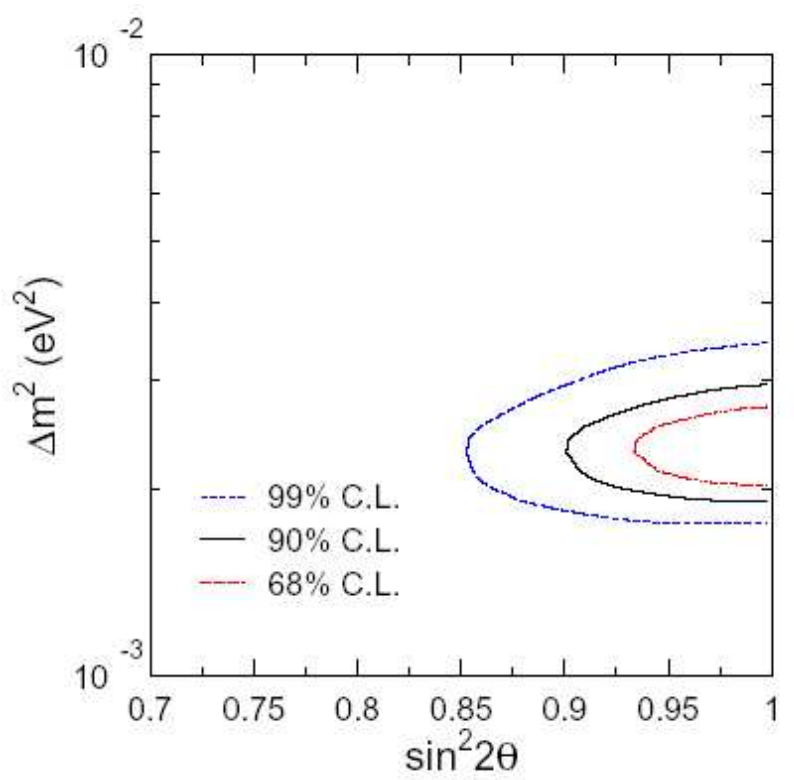

Figure 1.6: Allowed oscillation parameter space for 2-flavour $\nu_{\mu} \longleftrightarrow \nu_{\tau}$ oscillations obtained from Super-Kamiokande [10]

\subsection{Solar Neutrino Experiments}

The study of neutrinos thought to come from the sun has been of utmost interest and importance for many years. These studies provide an insight into neutrino oscillations, which will be covered in more detail, and the stellar interior for which it is, with the exception of helioseismology, the only probe. The sun produces neutrinos from internal nuclear fusion reactions. The produced solar neutrino energy spectrum spans the $\mathrm{keV} \rightarrow \mathrm{MeV}$ range as can be seen in Figure 1.9. The chemical process chain can be seen in Figure 1.8 .

There are two main experimental techniques used in solar neutrino experiments : radiochemical and liquid-Cherenkov detection. The principle of the radiochemical experiments is the 


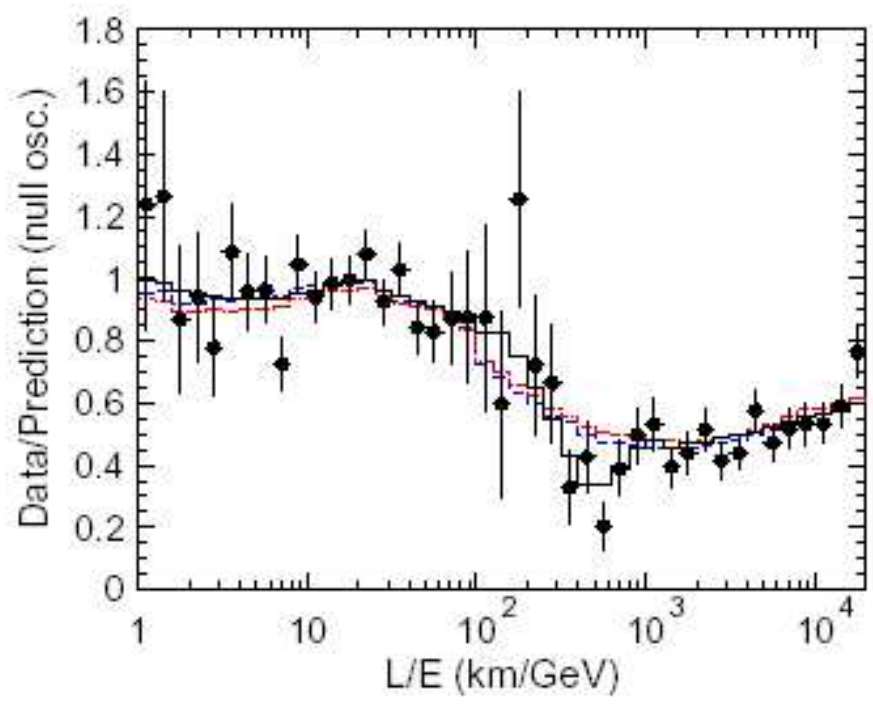

Figure 1.7: Ratio of the measured Super-Kamiokande data [11] to the Monte Carlo events without oscillation (points) as a function of reconstructed L/E together with the best-fit expectation for 2-flavour $\nu_{\mu} \longleftrightarrow \nu_{\tau}$ oscillations (solid black line). Also shown are the best-fit expectation for neutrino decay (dashed blue line) and decoherence (red dotted line). The errors are statistical.

reaction ${ }_{N}^{A} Z+\nu_{e} \rightarrow{ }_{N-1}^{A}(Z+1)+e^{-}$. The incident neutrino is captured producing an unstable daughter nucleus. The radioactive decay of this daughter nucleus is detected later. The incident neutrino flux from the sun at the surface of the earth is high (about $10^{10} \mathrm{~cm}^{-2} \mathrm{~s}^{-1}$ ), however the cross-section is very low at roughly $10^{-45} \mathrm{~cm}^{2}$ thus very large detectors of the order of many tons are required to achieve a transmutation rate of one atom/day.

The alternative detection method is real-time experiments. Generally this involves the detection of Cherenkov light as the neutrino scatters off electrons or reacts with deuterium in heavy water. These detectors have an energy threshold of around $5 \mathrm{MeV}$ making them only sensitive to the highest energy neutrinos produced by the sun. These neutrinos are produced by the ${ }^{8} B$ reaction ${ }^{1}$.

The first experimental observation of solar neutrinos was in 1968 at the Homestake Experiment[14] by Ray Davis Jr. This work led to him receiving the Nobel prize many years later. The Homestake experiment consisted of a large detection tank filled with 615tons of the common cleaning fluid $\mathrm{C}_{2} \mathrm{Cl}_{4}$ (tetrachloral ethene). The incident neutrinos underwent the

\footnotetext{
${ }^{1}$ Borexino has started data taking. This experiment has a lower threshold sensitivity to ${ }^{7} \mathrm{Be}$ neutrinos [13].
} 


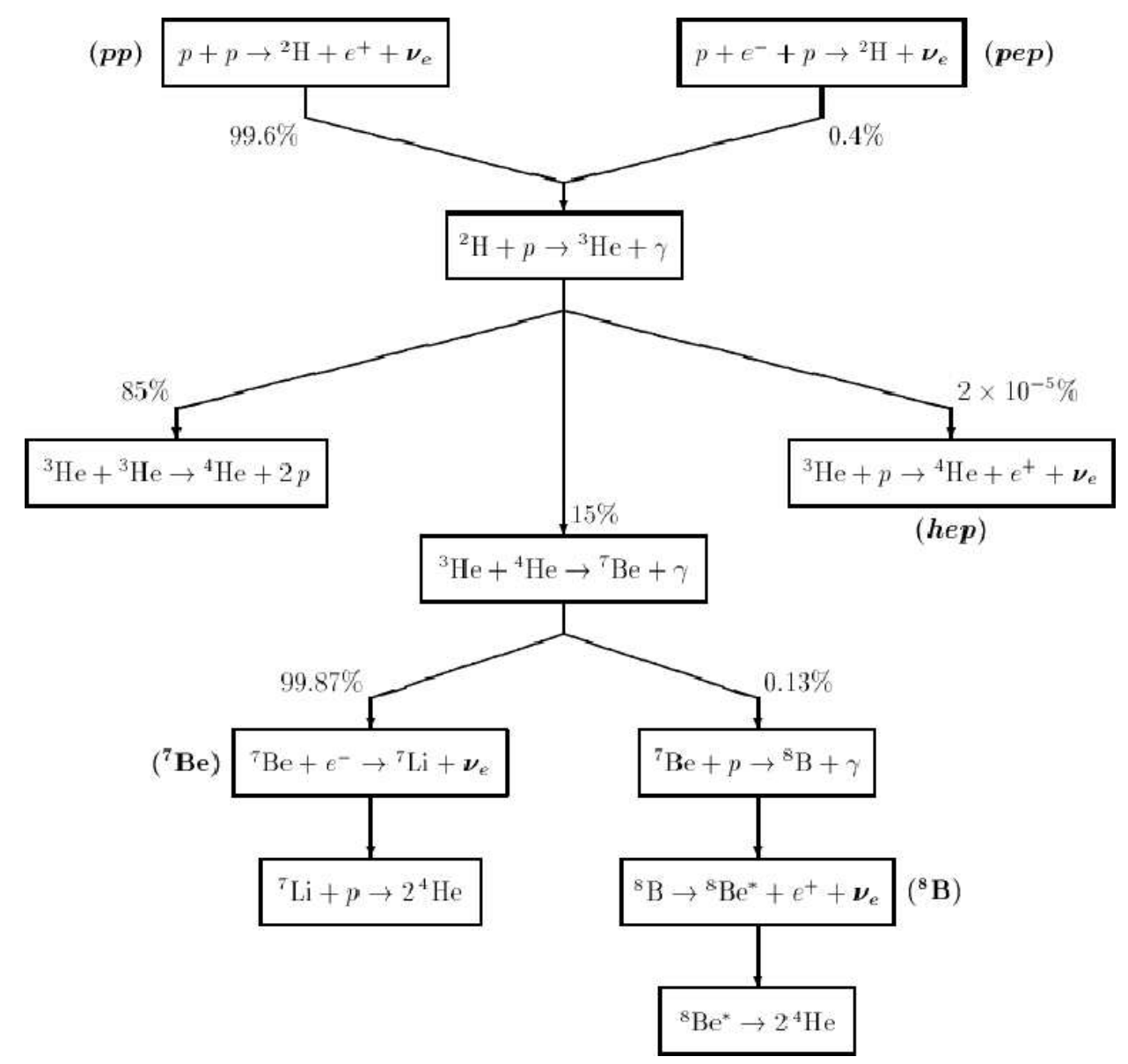

Figure 1.8: Solar PP cycle reaction chain with branching fractions

following reaction in the target:

$$
\nu_{e}+{ }^{37} \mathrm{Cl} \rightarrow e^{-}+{ }^{47} \mathrm{Ar}
$$

After each observing run of 2 months, the argon atoms were chemically extracted from the $C_{2} C l_{4}$ and the solar neutrino flux inferred from the number of argon atoms present. The experiment ran for around 30 years and results show a deviation in $\nu_{e}$ flux of approx. $1 / 3$ of the expected flux predicted by the Standard Solar Model (SSM) [15].

Two further radiochemical experiments, SAGE and GALLEX were constructed. Both these experiments used the reaction:

$$
\nu_{e}+{ }^{71} G a \rightarrow e^{-}+{ }^{71} G e
$$




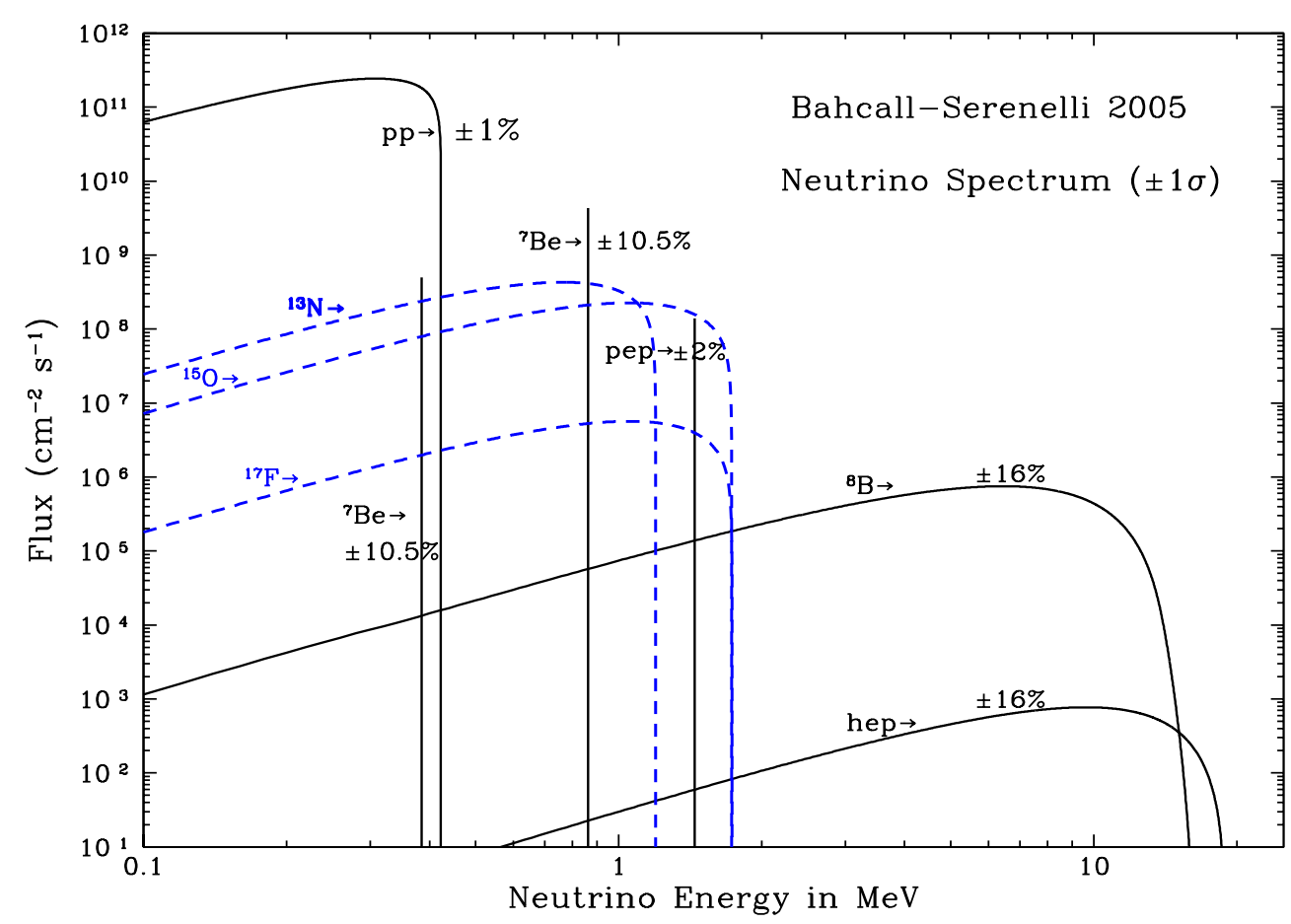

Figure 1.9: Standard Solar Model (SSM) fluxes for solar neutrinos produced from different reaction branches $[12]$

This reaction can capture the low energy pp neutrinos. This is important as the pp reactions are the key energy production mechanism in the sun, the Homestake experiment for example only measured neutrinos from a side-branch of the pp cycle. Both the results from SAGE and the results from GALLEX confirm the deficit in solar $\nu_{e}$. The SAGE [16] experiment analysed data from 92 runs during a 12 year period from January 1990 to December 2001 yielding the result $70.8_{-5.2}^{+5.3}(\text { stat })_{-3.2}^{+3.7}$ (sys) SNU where 1 SNU (Solar Neutrino Unit) $=10^{-36}$ captures/s/absorber nucleus. The SSM predicts this rate should be $128_{-7}^{+9}$ SNU. The GALLEX [17] experiment operated between 1991-97 with 65 gallium extractions yielding $320{ }^{71} \mathrm{Ge}$ decays and the result of $77.5 \pm 6.2(\text { stat })_{-4.7}^{+4.3}$ (sys) SNU. Again this should be compared to the SSM rate of $128_{-7}^{+9}$ SNU.

The detection methods adopted in the Homestake, SAGE and Gallex experiments provide no directional information for the detected neutrino. The next generation of neutrino experiments were required to provide directional information and to verify conclusively that these neutrinos were produced in the solar interior. The Kamiokande and Super-Kamiokande water Cherenkov detectors operated during the 1990s. Briefly, both detectors observed solar neutrinos via the following electron-neutrino elastic scattering: 


$$
\nu_{e}+e^{-} \rightarrow \nu_{e}+e^{-}
$$

The Cherenkov light produced by the highly relativistic scattered electron is detected. This provides the angular distribution of electrons scattered, which in turn is highly correlated to the direction of the incoming neutrino. The results confirming that these neutrinos are indeed solar neutrinos can be seen in Figure 1.10.

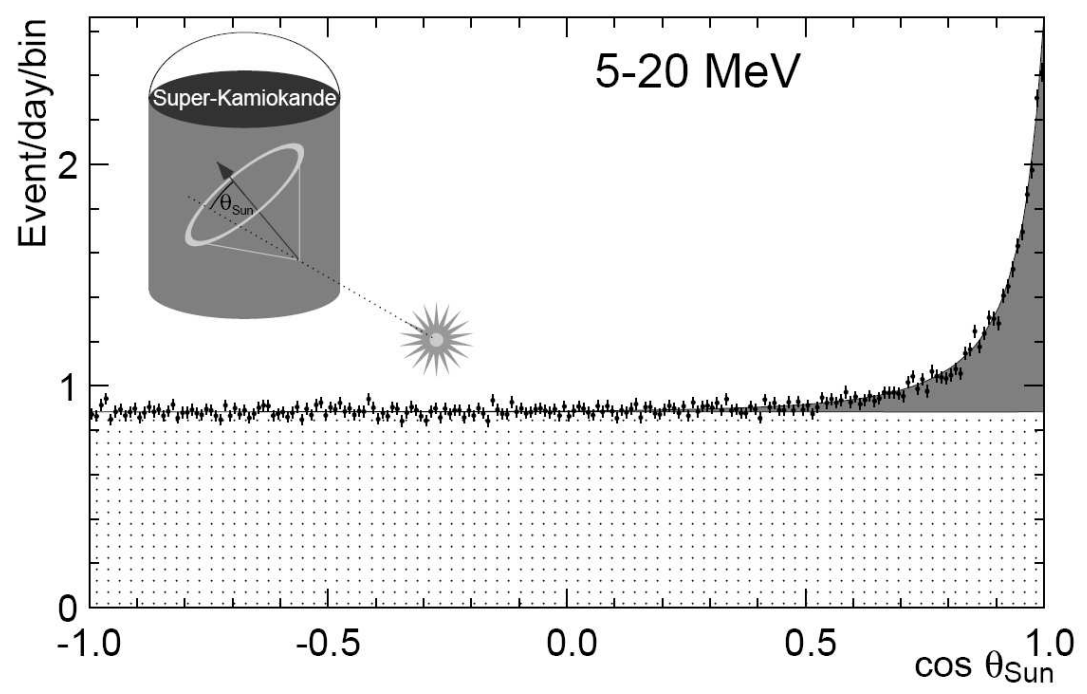

Figure 1.10: $\cos \left(\theta_{\text {sun }}\right)$ distribution of solar neutrino event candidates from the SuperKamiokande I data set (1496 days). [18]

All the solar neutrino experiments discussed have observed a statistically significant deficit of neutrinos compared with the expected theoretical SSM rates. This deficit has been proposed to be explained by neutrino flavour oscillation of the original $\nu_{e}$ produced in the sun. Other theories have been postulated such as neutrino decay and decoherence [19]. The verification that the total neutrino flux for all flavours was conserved and thus that the deficit of solar $\nu_{e}$ was attributed to flavour oscillation was provided by the Sudbury Neutrino Observatory (SNO) experiment in Canada. The results from this experiment also verified the SSM as the correct description of our sun explaining fully the so-called "solar neutrino problem". A result that caused the theorist who formulated the SSM, the late John Bahcall, to "want to dance" after nearly 30 years of the community scrutinising his calculations because of the poor agreement to the measured neutrino flux. 
The SNO detector is a large 1000 tonne heavy-water $\left(D_{2} O\right)$ Cherenkov detector placed $2 \mathrm{~km}$ underground in the Creighton mine near Sudbury, Ontario, Canada. SNO is sensitive to $\nu_{e}, \nu_{\mu}$ and $\nu_{\tau}$ via neutral currents with a detection threshold of roughly $6 \mathrm{MeV}$ hence sensitive to mainly ${ }^{8} B$ neutrinos. SNO makes use of 3 important reactions $\left(\nu_{x}\right.$ refers to any active flavour neutrino).

$\begin{array}{ll}\text { Elastic Scattering (ES) } & \nu_{e}+e^{-} \rightarrow \nu_{x}+e^{-} \\ \text {Charged Current (CC) } & \nu_{e}+d \rightarrow p+p+e^{-} \\ \text {Neutral Current (NC) } & \nu_{x}+d \rightarrow n+p+\nu_{x}\end{array}$

The neutrino-electron elastic scattering couples to all flavours of neutrino and gives good directional sensitivity through the detection of Cherenkov light produced by the scattered electron. In the CC interaction only $\nu_{e}$ 's couple significantly to the $\mathrm{Z}$ boson. This couples to one of the quarks in the deuterium neutron producing a proton. This causes dissociation of the nucleus and the $e^{-}$produced in the weak interaction is again detected via its Cherenkov cone. The NC interaction has the same sensitivity to all neutrino flavours, causing dissociation of the nucleus with the free neutron detected. This channel is able to measure the total solar neutrino flux.

The second stage of SNO began in 2001 when 2 tons of $\mathrm{NaCl}$ were added to the heavy water. This addition of salt enhanced the signal from neutron capture compared with pure $\mathrm{D}_{2} \mathrm{O}$. The calculated neutrino fluxes can be seen in Table 1.1.

\begin{tabular}{c|c} 
Interaction & Flux $\left(\times 10^{6} \mathrm{~cm}^{2} \mathrm{~s}^{-1}\right)$ \\
\hline \hline Elastic Scattering & $2.35_{-0.22}^{+0.22}(\text { stat })_{-0.15}^{+0.15}(\mathrm{sys})$ \\
Charged Current & $1.60_{-0.06}^{+0.06}$ (stat $)_{-0.09}^{+0.08}(\mathrm{sys})$ \\
Neutral Current & $4.94_{-0.21}^{+0.21}$ (stat $)_{-0.34}^{+0.38}$ (sys $)$
\end{tabular}

Table 1.1: Calculated neutrino flux from the salt phase of the SNO detector

The SNO NC result is consistent with the SSM, with the CC channel results providing around $1 / 3$ of the predicted SSM flux $\nu_{e}$. This shows that the solution to the solar neutrino problem is $\nu_{e} \rightarrow \nu_{x}$ flavour oscillation. These results are consistent with a Large-Mixing-Angle (LMA) (Figure 1.11). A combined analysis of all solar neutrino experiments yields the following values for the solar oscillation parameters $\left(\Delta m_{\text {sol }}^{2}, \tan ^{2} \theta_{\text {sol }}\right)[20]$ : 


$$
\begin{gathered}
\Delta m_{\text {sol }}^{2}=6.5_{-2.3}^{+4.4} \times 10^{-5} \mathrm{eV}^{2} \\
\tan ^{2} \theta_{\text {sol }}=0.45_{-0.08}^{+0.09}
\end{gathered}
$$
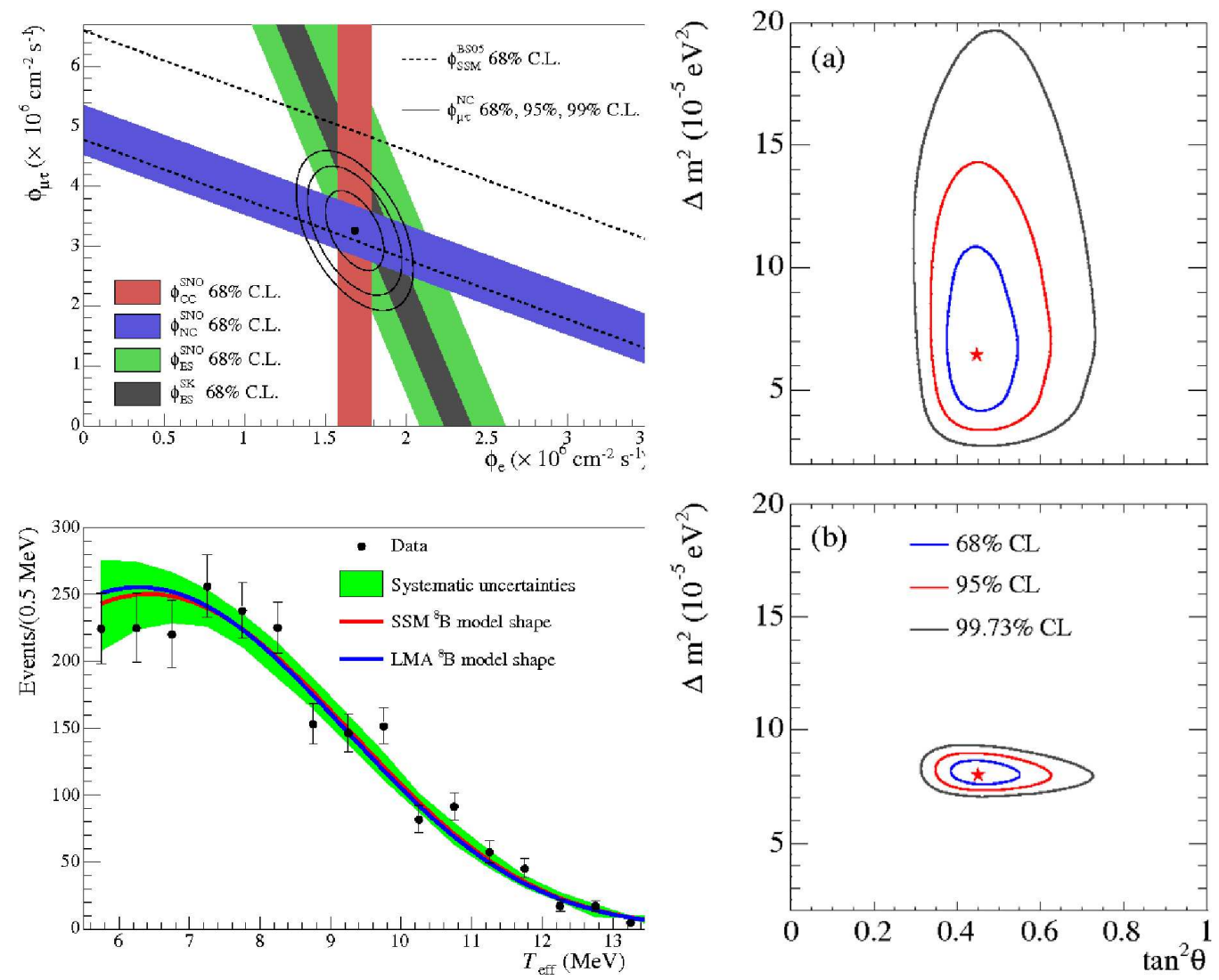

Figure 1.11: Top upper: The flux of $\mu+\tau$ neutrinos versus flux of electron neutrinos. CC, NC and ES fluxes are indicated by solid bands. The ${ }^{8} B$ SSM solar flux is indicated by the dashed lines. The narrow band parallel to the SNO ES result corresponds to the Super-Kamiokande result. Right upper and lower: Global neutrino oscillation analysis using only solar neutrino data (a) and including KamLAND 766 ton-year data (b). Left lower: Charged current $T_{\text {eff }}$ spectrum with the best fit LMA parameters. A $1 \sigma$ uncertainty on the undistorted ${ }^{8} B$ model shape determined by detector systematics is indicated by the green band. The predicted spectrum is normalised to the same number of counts as the data spectrum [20].

\subsection{Reactor Neutrino Experiments}

Reactor neutrino experiments have been important in establishing our current state of knowledge of the neutrino. As discussed previously, the discovery of the neutrino was made at the 

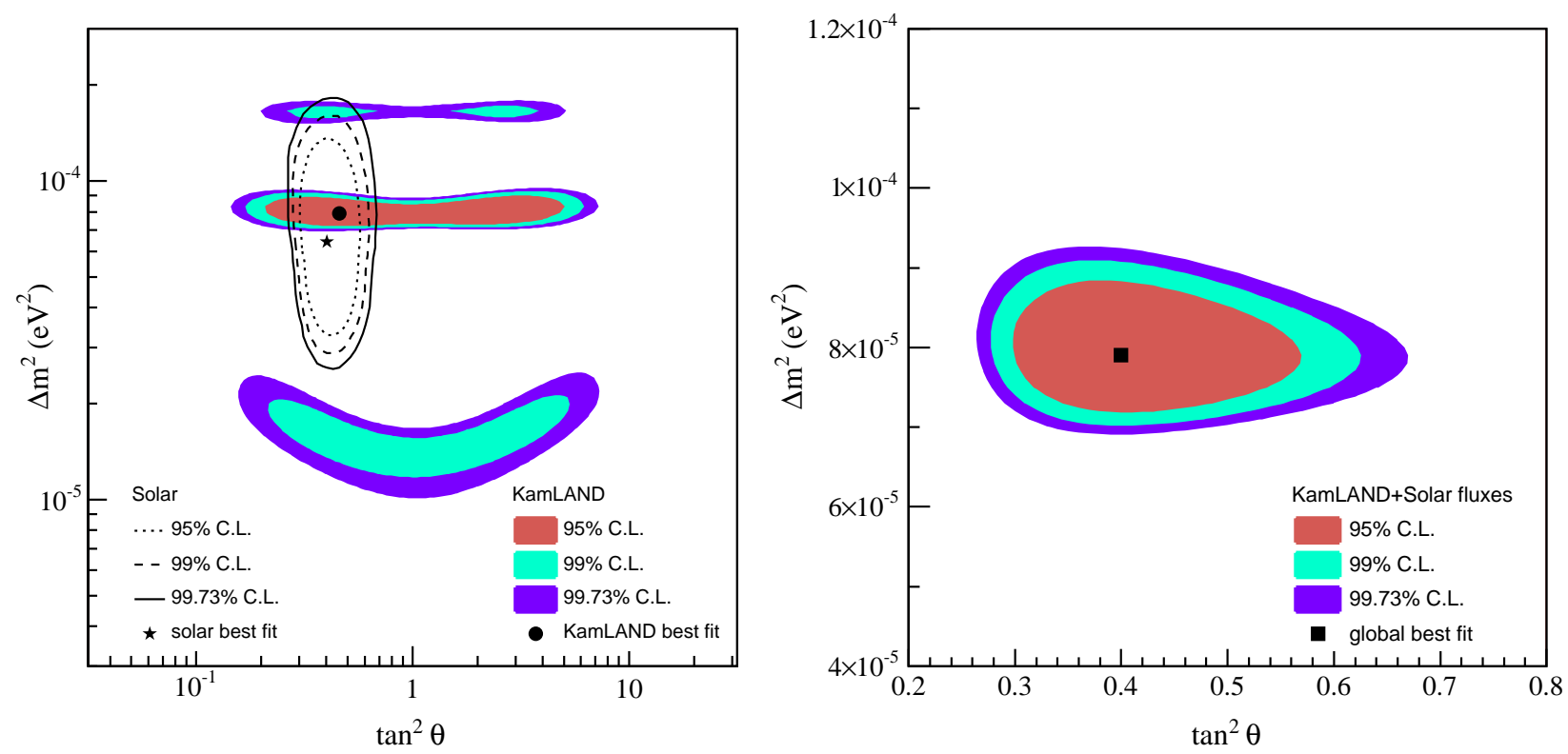

Figure 1.12: (a) Allowed region in oscillation parameter space from KamLAND anti-neutrino data (shaded regions) and solar neutrino experiments (lines). (b) Result of combined two-flavour oscillation analysis of KamLAND and the observed solar fluxes under the assumption of CPT invariance. The fit gives $\Delta m^{2}=7.9_{-0.5}^{+0.6} \times 10^{-5}$ and $\tan ^{2} \theta=0.40_{-0.07}^{+0.10}[21]$.

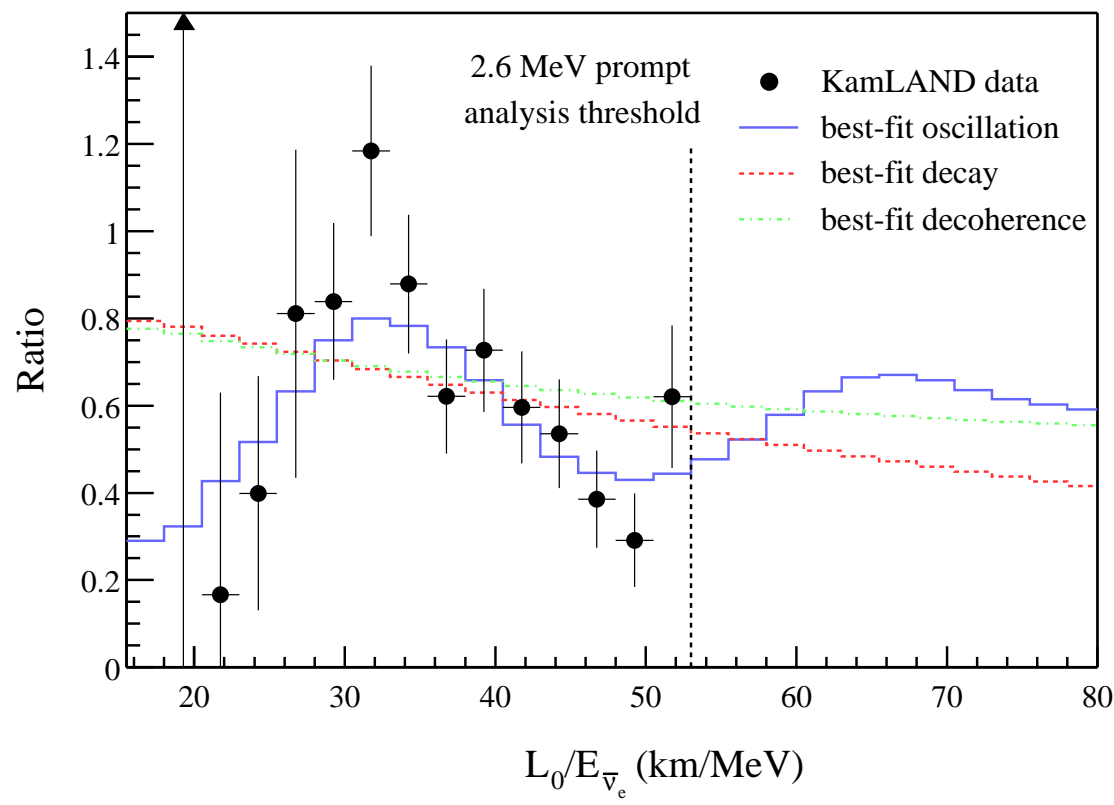

Figure 1.13: Event distribution in $L_{0} / \mathrm{E}$, where $L_{0}$ is taken to be $180 \mathrm{~km}$. The solid blue curve is the best fit oscillation spectrum. The two remaining curves show the dependence on $L_{0} / \mathrm{E}$ for neutrino decay and decoherence theories [19].

Savannah River reactor experiment in 1956. Since then, reactor experiments have continued to be influential in probing neutrino parameters. 
The anti-neutrinos produced in nuclear reactors via $\beta^{-}$decay of neutrons during nuclear fission processes can be used to study both the solar mass difference $\left(\Delta m_{12}^{2} \approx 8 \times 10^{-5} \mathrm{eV}^{2}\right)$ and atmospheric mass difference $\left(\Delta m_{13}^{2} \approx 2.5 \times 10^{-3} \mathrm{eV}^{2}\right)$. Copious numbers of neutrinos are produced in today's reactors e.g. ${ }^{235} U$ fission in a reactor producing $\approx 3 G W$ of thermal power produces a neutrino flux of the order $6 \times 10^{20} \bar{\nu}_{e} / \mathrm{sec}$. The neutrino production and interactions are very well known in reactor experiments. This results in these experiments being almost unaffected by the dominant uncertainty in most current neutrino oscillation experiments, which is the initial flux and content of the beam [22]. Reactor neutrino experiments are disappearance experiments with close to mono-chromatic beams of mean neutrino energy $\approx 3.6 \mathrm{MeV}$. To probe the different mass scales it is therefore necessary to pick different baselines. For example, at this energy the oscillation peak for $\Delta m_{12}^{2} \approx 8 \times 10^{-5} \mathrm{eV}^{2}$ requires a baseline of $\approx 1.8 \mathrm{~km}$ and $\Delta m_{13}^{2} \approx 2.5 \times 10^{-3} \mathrm{eV}^{2} \approx 60 \mathrm{~km}$.

The value of $\theta_{13}$ is small and possibly zero. If zero, this would exclude the possibility that we have $\mathrm{CP}$ violation in the lepton sector. If non-zero, the value will be central to the design of future experiments needed to resolve the mass hierarchy problem and search for CP violation. The most important experiment that set limits on the value of $\theta_{13}$ is the CHOOZ experiment. CHOOZ is located in the Ardennes region of France and is a two reactor power station with a total thermal power of $8.5 \mathrm{GW}$. The $\mathrm{CHOOZ}$ detector is a 5 ton, Gd-loaded liquid scintillator experiment displaced $1 \mathrm{~km}$ from the reactor and 300 meters-water-equivalent underground. The neutrino is detected in the inverse beta decay interaction:

$$
\bar{\nu}_{e}+p \rightarrow n+e^{+}
$$

By looking for the coincidence of the prompt positron signal with a delayed neutron capture in the Gd-loaded scintillator according to the reaction:

$$
n+{ }^{m} G d \rightarrow{ }^{m+1} G d^{*} \rightarrow^{m+1} G d+\gamma^{\prime} s(8 M e V)
$$

The results from the CHOOZ run $4 / 1997 \rightarrow 7 / 1998$ show no evidence for $\bar{\nu}_{e}$ disappearance at the $90 \%$. The current world limit is $\sin ^{2}\left(2 \theta_{13}\right)<0.15$ for $\Delta m_{31}^{2}$. This is plotted in Figure 1.14. There are many proposals for future experiments which will aim to improve on this 
value of $\sin ^{2}\left(2 \theta_{13}\right)$, which include the Angra, Double Chooz, Daya Bay, KASK and KR2DET experiments. These will not be discussed, although for a comprehensive overview with more detailed references the reader is pointed to [23].

The KamLAND experiment [24] has also played a crucial role in constraining neutrino oscillation parameters, specifically oscillations at $m_{\text {solar }}^{2}$ KamLAND, located in Japan, is a relatively long baseline experiment compared to previous reactor experiments. The detector is a $1.2 \mathrm{kton}$ ultra-pure liquid scintillator detector balloon housed 2700 metres-water-equivalent underground. The reactors supplying neutrinos to the detector are distributed around Japan and South Korea. There are 53 reactors with varying baselines, however $80 \%$ of the neutrino flux comes from reactors distributed from 130-220 km which in turn gives an effective baseline of approximately $180 \mathrm{~km}$. This is an important baseline as it is the correct distance to probe the Large-MixingAngle (LMA) solution for neutrino oscillations, which is accepted as the most favourable solution to the solar oscillation parameter.

KamLAND detects the anti-neutrino again by a coincidence between prompt positron emission in the inverse beta reaction and the delayed neutron capture by hydrogen. The results from KamLAND [25] show that the ratio of observed reactor $\bar{\nu}_{e}$ events to those expected assuming no neutrino oscillation is:

$$
\frac{N_{\text {obs }}-N_{B G}}{N_{\text {expected }}}=0.611 \pm 0.085(\text { stat }) \pm 0.041 \text { (syst) }
$$

This result is compared with results from previous reactor experiments in Figure 1.15. All solar parameter regions are excluded except the LMA region. The oscillation parameter region allowed by KamLAND is combined with the solar neutrino data to give the current world-best values for the solar neutrino parameters previously shown in Figure 1.12. It can be seen from Figure 1.13 which shows the distribution of Kamland events as a function of $L_{0} / E_{\bar{\nu}_{e}}$ that the oscillation fit is favoured over other models.

\subsection{Accelerator Neutrino Experiments}

Neutrino experiments utilising neutrinos produced from accelerator complexes will undoubtedly prove to be the future of neutrino physics. Precision determination of intrinsic neutrino 


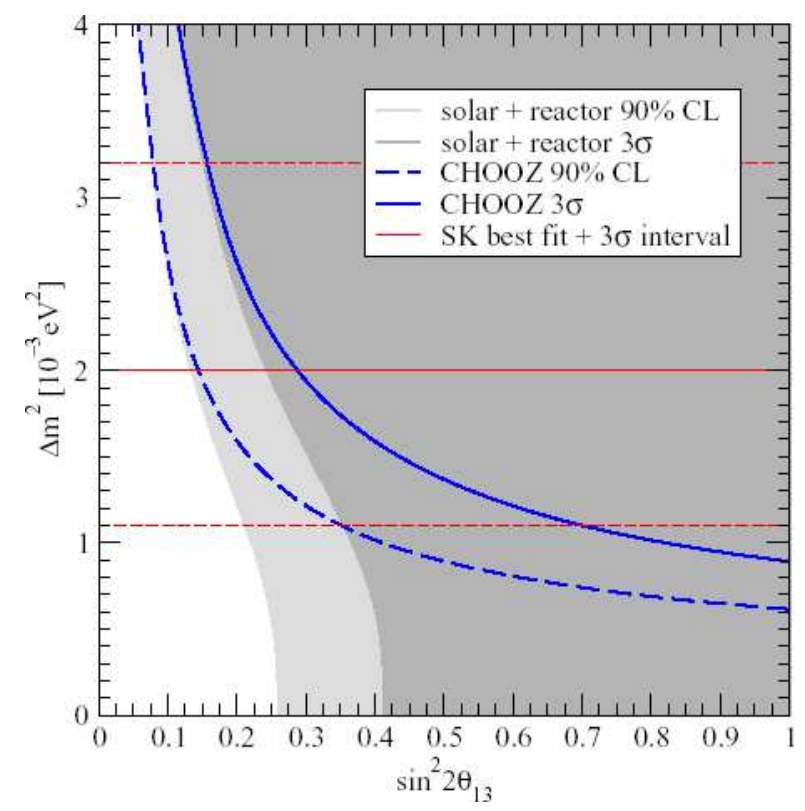

Figure 1.14: Upper bound on $\sin ^{2}\left(2 \theta_{13}\right)$ from the CHOOZ experiment at $90 \%$ (dashed) CL and $3 \sigma$ (solid) CL for 1 DOF as a function of $\Delta m_{31}^{2}$. The light (dark) shaded region is excluded from $\mathrm{CHOOZ}+$ solar+KamLAND data at $90 \%(3 \sigma)$ CL for 1 DOF. The horizontal lines indicate the current best-fit value and the $3 \sigma$ allowed regions for $\Delta m_{31}^{2}[23]$

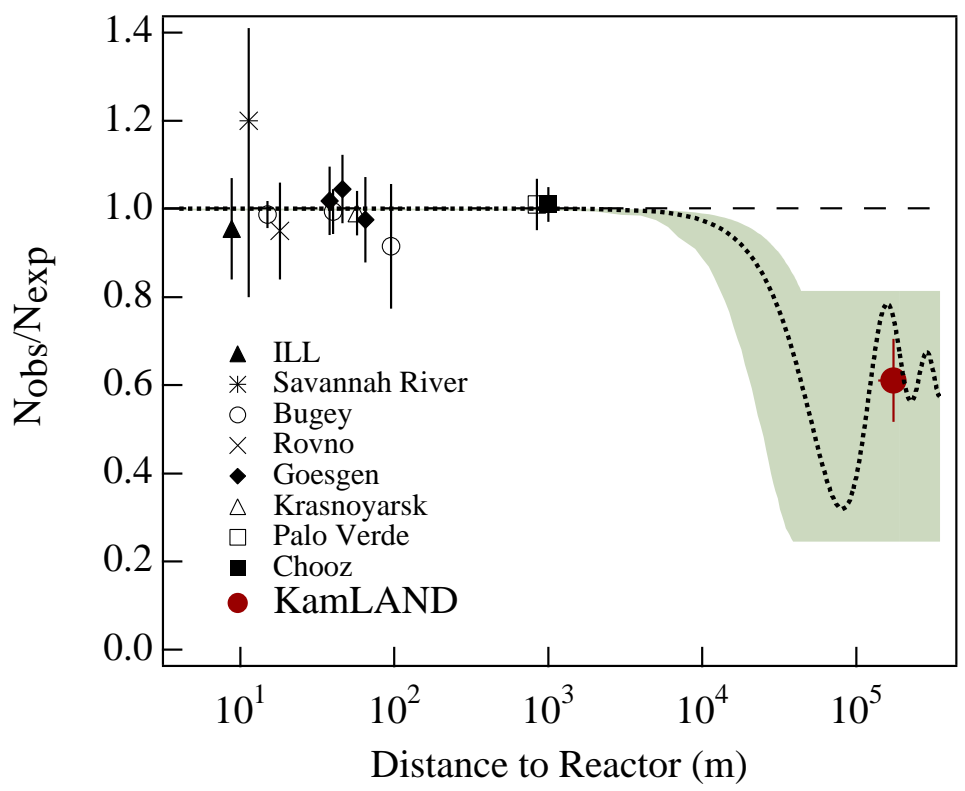

Figure 1.15: The ratio of measured to expected $\bar{\nu}_{e}$ flux from reactor experiments. The solid circle denotes the KamLAND result plotted at the flux averaged distance of approximately $180 \mathrm{~km}$. The shaded region indicates the flux prediction corresponding to the 95\% CL LMA region parameter space from a global analysis of solar neutrino data. The dotted line is representive of a best-fit LMA prediction $\left(\sin ^{2} 2 \theta=0.833\right.$ and $\Delta m^{2}=5.5 \times 10^{-5} \mathrm{eV}^{2}$ The dashed line is the expected flux ratio under the no oscillation hypothesis [25] 
parameters in the future will require a large investment in a new accelerator complex.

Previous accelerator experiments have already played an important part in recent studies on neutrino oscillations. The Liquid Scintillator Neutrino Detector (LSND)[26] conducted a data run from 1993-1998 and was situated at Los Alamos National Laboratory (LANL). LSND consisted of a $167 \mathrm{t}$ cylindrical mineral-oil detector, $8.3 \mathrm{~m}$ length by $5.7 \mathrm{~m}$ diameter which used both scintillation and Cherenkov light for detection. A $800 \mathrm{MeV}$ kinetic energy proton beam impinged on a $30 \mathrm{~cm}$ long water target located $1 \mathrm{~m}$ upstream of a copper beam stop. The majority of the $\pi^{+}$produced are stopped in the target. The subsequent decay muons also mostly decay in the target. The $\bar{\nu}_{\mu} \rightarrow \bar{\nu}_{e}$ oscillation was investigated by studying the anti-neutrinos produced from the muon decay. In addition the $\nu_{\mu} \rightarrow \nu_{e}$ oscillation was studied using neutrinos produced by the pion population(roughly $3 \%$ ) which decay in the $1 \mathrm{~m}$ drift space between target and beam stop. Analysis of the $\bar{\nu}_{\mu} \rightarrow \bar{\nu}_{e}$ channel yielded a result of $87.9 \pm 22.4 \pm 6.0$ excess events that were measured. By assuming the oscillation hypothesis this yields an oscillation probability of $\mathrm{P}\left(\bar{\nu}_{\mu} \rightarrow \bar{\nu}_{e}\right)=2.64 \pm 0.68 \pm 0.45 \times 10^{-3}$. This result suggests a $\Delta m^{2} \approx 1 \mathrm{eV}^{2}$. The full parameter region can be seen in the upper upper part of Figure 1.19. This mass-squared difference is not consistent with the solar or atmospheric neutrino data. It requires at least one new species of neutrino which has the property of not being weakly interacting. The absense of a charged lepton partner excluding interactions mediated by the $\mathrm{W}$ boson and the width of the $Z_{0}$ peak at LEP mentioned earlier excludes interactions via the Z. They are therefore termed sterile. A similar parameter region to LSND was probed with the KARMEN experiment at the RutherfordAppleton laboratory in the UK. KARMEN ran from 1990 to 2001 and used the ISIS 800MeV proton beam to produce a neutrino spectrum almost identical to the LSND beam. The detector was a 56t liquid scintillator detector placed at 18m from the target. In February 1997 a second data run commenced after installation of a background reducing atmospheric muon veto shield, this was termed KARMEN2. The $\bar{\nu}_{\mu} \rightarrow \bar{\nu}_{e}$ analysis on the data collected during the KARMEN2 phase gave 15 candidate events with an expected background of 15.8 events. This suggests no clear evidence for neutrino oscillations in this regime and excludes some, but not all of the LSND parameter space, see Figure 1.19.

An experiment called MiniBoone, designed to confirm or refute the LSND results is currently taking data. The MiniBoone experiment will probe $\nu_{\mu} \rightarrow \nu_{e}$ oscillations at a baseline of $\approx 500 \mathrm{~m}$. 
The muon-neutrinos are produced from decay of pions created by an $8 \mathrm{GeV}$ proton beam from the Fermilab Booster incident on a Be target. The first results from MiniBoone have very recently been released [27]. Initial analysis of the data (see Figure 1.16) suggests there are no effects present other than the two neutrino $\nu_{\mu} \rightarrow \nu_{e}$ oscillation. This disfavours the hypothesis that the LSND result is due to two neutrino appearance only oscillation at 98\% CL.

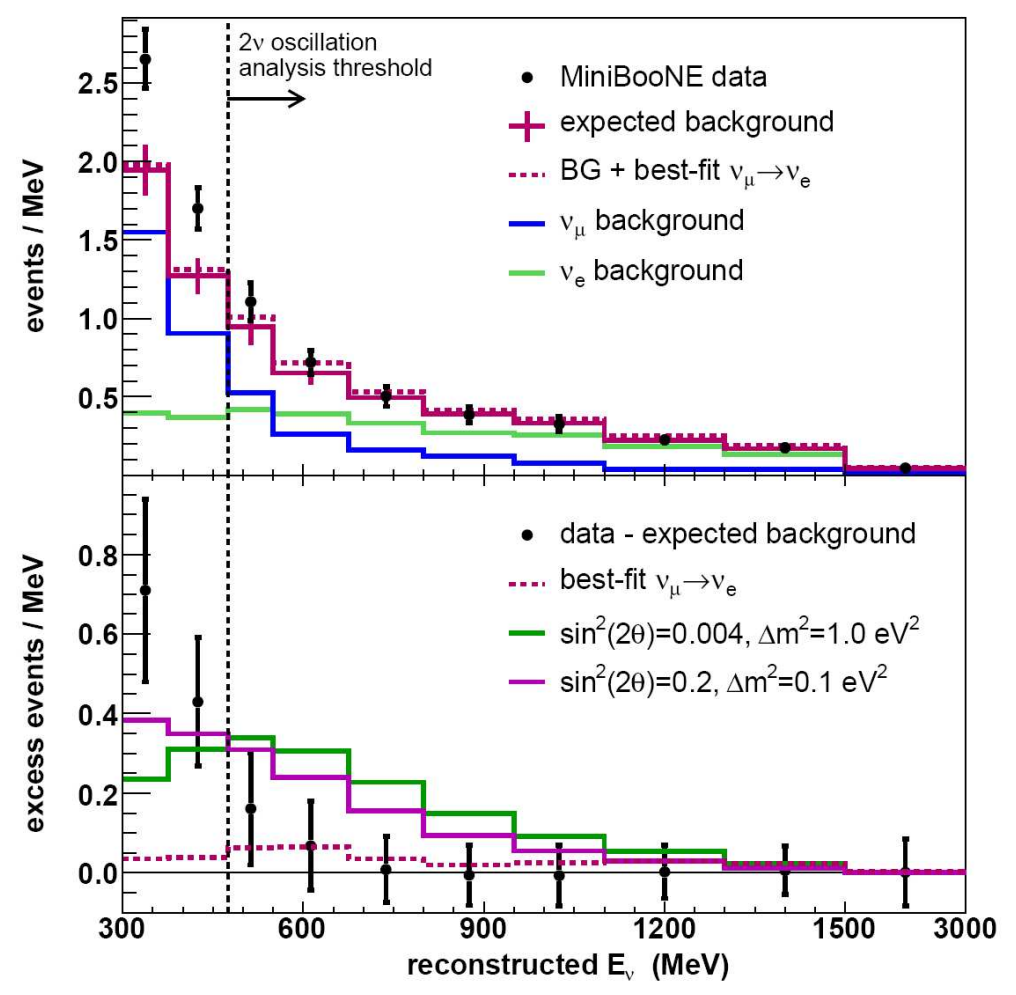

Figure 1.16: The upper plot shows the number of candidate $\nu_{e}$ events as a function of $E_{\nu}^{Q E}$. The points represent the data with statistical error, whereas the histogram shows the expected background with systematic errors from all sources. The vertical dashed line shows the threshold used in the two-neutrino oscillation analysis. Also shown are the best-fit oscillation spectrum (dashed histogram) and the background contributions from $\nu_{\mu}$ and $\nu_{e}$ events. The bottom panel shows the number of events with the predicted background subtracted as a function of $E_{\nu}^{Q E}$, where the points represent the data with total errors and the two histograms correspond to LSND solutions at high and low $\Delta m^{2}[27]$

Accelerator neutrino facilities will play a crucial role in the future precision measurement of neutrino parameters. Future facilities generally fall into three different categories:

- Conventional Super-beams

- Beta-beams

- Neutrino Factory 
The first category is an extension of existing neutrino beam technology. The conventional neutrino production via $\pi^{ \pm} \rightarrow \mu^{ \pm}$will be optimised for a very high intensity and low-energy $\nu_{\mu}$ flux. Facilities of this sort are known collectively as neutrino Superbeams. Current conventional beam facilities include the Mini-Boone experiment which has already been discussed and the $\mathrm{K} 2 \mathrm{~K}$ project. The $\mathrm{K} 2 \mathrm{~K}$ experiment is a conventional beam experiment where $|E|=1.4$ $\mathrm{GeV}$ neutrinos are produced from pion and kaon decay from interactions of $12 \mathrm{GeV}$ protons produced by the KEK proton synchrotron in Kamioka, Japan. The neutrino beam is detected using two neutrinos at different baselines. The near detector is located at the KEK site and is used to measure cross-sections and beam spectra. The far detector is the SuperKamiokande water cherenkov detector located at $\mathrm{L}=250 \mathrm{Km}$. The $\mathrm{K} 2 \mathrm{~K}$ experiment will be used to verify the atmospheric oscillation result already mentioned.

The MINOS experiment [28] consists of a near and far detector at baselines of $1 \mathrm{~km}$ and $735 \mathrm{~km}$ respectively from the graphite target at Fermilab, Chicago. Initial results accumulated in a run from May 2005 to February 2006 by the MINOS experiment (Main Injector Neutrino Oscillation Experiment) observed 215 events below $30 \mathrm{GeV}$ in the far detector at $735 \mathrm{~km}$ from the target. When this is compared to the expected $336 \pm 14.4$ events it suggests $\left.\nu_{(} \mu\right)$ oscillation with $\left|\Delta m^{2}\right|=2.74_{-0.26}^{+0.44} \times 10^{-3} \mathrm{eV}^{2} / \mathrm{c}^{4}$ and $\sin ^{2}\left(2 \theta_{23}\right)>0.87$ (68\% C.L.). The MINOS experiment will run for 5 years. This will hopefully improve the knowledge of the atmospheric neutrino parameters even further.

The CNGS experiment is a $\nu_{\tau}$ appearance experiment [29]. The neutrino beam is produced in CERN and is detected at the Gran Sasso Laboratory a distance of $732 \mathrm{~km}$ away. Protons produced by the CERN SPS with energies of $450 \mathrm{GeV}$ impinge on a graphite target. The pions produced are focussed by a magnetic horn into a $1 \mathrm{~km}$ decay pipe

The next generation of long base-line experiments will aim to increase precision on atmospheric neutrino parameters and probe lower values of $\sin ^{2} 2 \theta_{13}$. The two experiments proposed to probe these parameters in the medium term are the T2K and NOVA experiments. The $\mathrm{T} 2 \mathrm{~K}$ experiment[30] is a next generation long baseline neutrino superbeam experiment which will use the high intensity JHF (Japanese Hadron facility, Tokai, Japan) 50 GeV proton synchrotron (PS) to produce a neutrino beam which will be fired at the Super-Kamiokande (SK) water Cherenkov detector. The JHF PS will deliver $\approx 3.3 \times 10^{14}$ protons every $3.5 \mathrm{~s}(0.75 \mathrm{MW})$. 


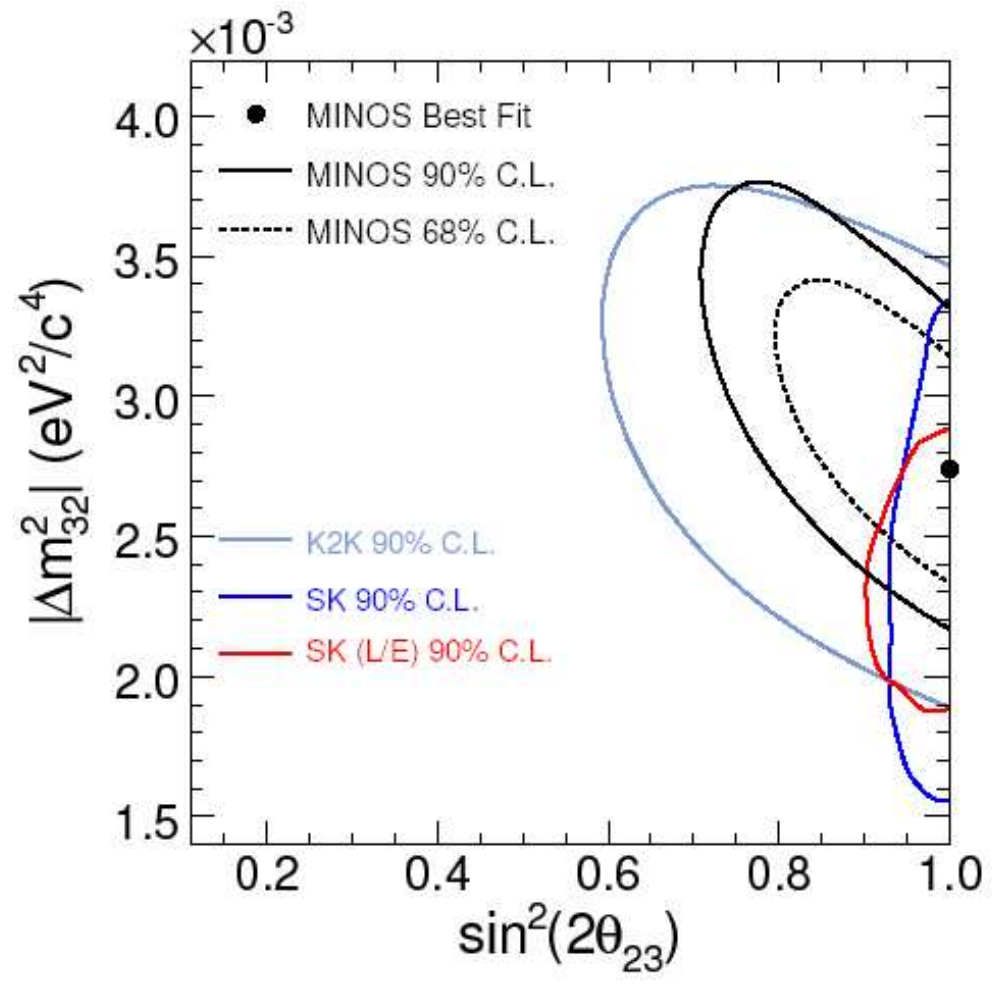

Figure 1.17: Atmospheric parameter confidence levels from the MINOS, K2K and SuperKamiokande experiments [28]

The neutrino beam produced will be utilised in an off-axis configuration with the SK detector lying $2^{\circ}$ degrees from the neutrino beam axis at a distance of $295 \mathrm{~km}$. This allows for a narrow pseudo mono-chromatic beam $(\approx 0.8 \mathrm{GeV})$ as a result of the pion decay kinematics. Assuming a run of 650 days $\mathrm{T} 2 \mathrm{~K}$ should improve the sensitivity to $\sin ^{2} 2 \theta_{13} \approx 0.006$ via the oscillation of $\nu_{\mu} \rightarrow \nu_{e}$ at $\Delta m^{2} \approx 3 \times 10^{-3} \mathrm{eV}^{2}$. T2K will also improve the precision on the atmospheric neutrino parameters to $\delta\left(\Delta m_{23}^{2}\right)=10^{-4}$ and $\delta\left(\sin ^{2}\left(2 \theta_{23}\right)\right)=0.01$.

A second stage of the experiment has been considered (T2HK) in which key upgrades will be made to improve performance. Specifically the proton driver would be upgraded to $4 \mathrm{MW}$. This would also be a key step toward a future Neutrino Factory complex. In addition, a new 1 mega-ton detector would be constructed in the Kamioka mine "Hyper-Kamiokande". This detector would have a rich physics program and will improve sensitivity to proton decay by extending the measurable lifetime to $10^{35}$ years. In terms of neutrino oscillations, if the first stage does discover the value for $\sin ^{2}\left(\theta_{13}\right)$ then T2HK will provide a further increase in $\nu_{\mu} \rightarrow \nu_{e}$ sensitivity and will be able to probe $\mathrm{CP}$ violation in the lepton sector. 
The future of the Fermilab neutrino program post-MINOS will be the experiment NOVA. The NuMI beamline will be utilised again to study $\nu_{\mu} \rightarrow \nu_{e}$ oscillations using a 30kT off-axis detector. NOVA will measure $\nu_{\mu} \rightarrow \nu_{e}$ oscillations to a precision of 10x MINOS. NOVA will also study the $\theta_{13}$ mixing angle and possibly the mass-hierarchy.

A relatively new concept which may yet prove to be a contender for a future precision neutrino measurements is the Beta Beam [31]. First proposed in 2002 [32] this facility is based on the acceleration to high energy of beta unstable ions. These ions would then consequently be injected into a decay ring where they would decay to very pure beams of $\nu_{e}$ or $\bar{\nu}_{e}$. The advantage of storing nuclides compared with the muon storage incorporated by a future neutrino factory facility is three-fold. Firstly, the energy distribution of $\beta^{ \pm}$is very well known providing excellent precision in the initial neutrino beam flux. Secondly, the energy of the beta decay is much lower than the corresponding muon decay and the produced neutrino beam has a low divergence. Finally, the nuclides in question have a relatively long lifetime of the order 1s. This eliminates the requirement for extremely fast acceleration required to accelerate the short-lived muons in a neutrino factory. The over-arching advantage of a beta-beam, however is that because it does not require this fast acceleration one could use conventional or existing accelerators.

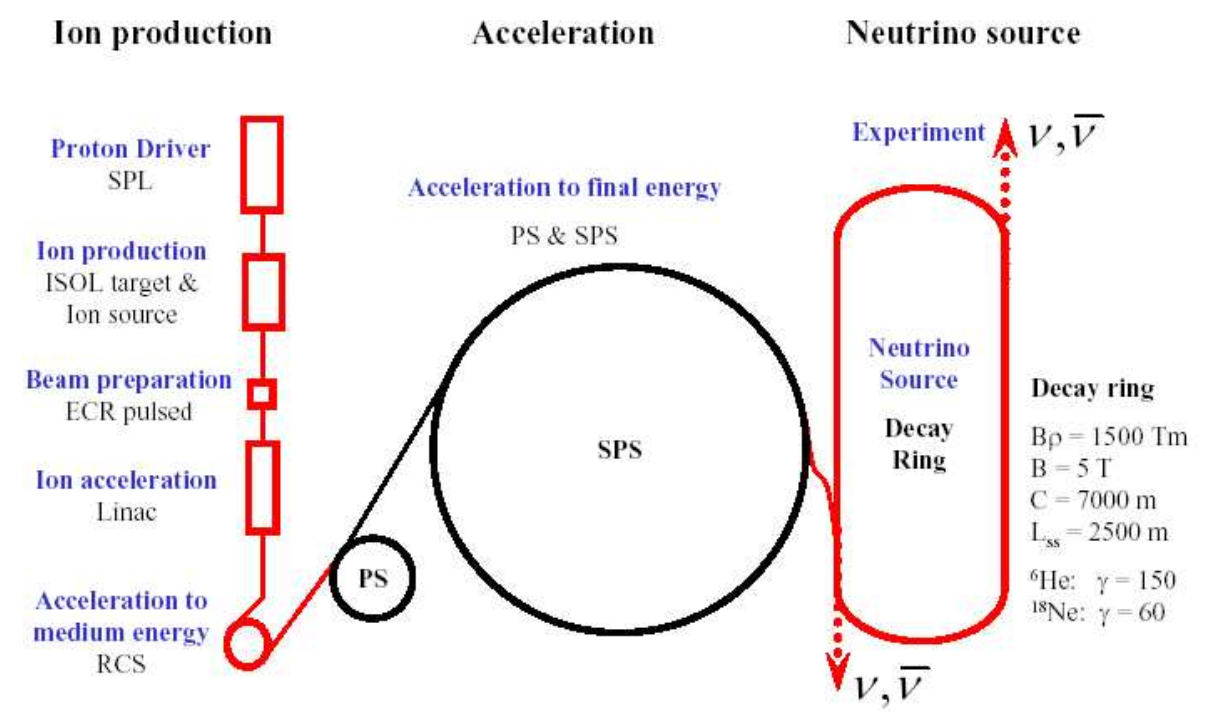

Figure 1.18: Schematic representation of the baseline beta-beam facility at the CERN SPS [33]

The baseline Beta-Beam study is incorporated under the umbrella of the larger context EURISOL study at CERN [34]. The EURISOL concept is to build a general next-generation facility for production of radioactive isotopes which in the low energy range includes precisely 
those required for a beta-beam facility. The choice of ions centres around the need to identify nuclides with not too short or long a half-life such that we require the correct intensity. Also important is the need to be able to produce significant quantities. A good compromise to satisfy these criteria is if one imagines the storage ring being populated by ${ }^{6} \mathrm{He}$ and ${ }^{18} \mathrm{Ne}$. These nuclides decay to $\overline{\nu_{e}}$ and $\nu_{e}$ respectively according to the following decays:

$$
\begin{gathered}
{ }_{2}^{6} \mathrm{He} \rightarrow{ }_{3}^{6} \mathrm{Li} e^{-\overline{\nu_{e}}} \quad \text { Average } E_{c m}=1.937 \mathrm{MeV} / \mathrm{c} \\
{ }_{10}^{18} \mathrm{Ne} \rightarrow{ }_{9}^{18} \mathrm{Fe} e^{+} \nu_{e} \quad \text { Average } E_{c m}=1.86 \mathrm{MeV} / \mathrm{c}
\end{gathered}
$$

A schematic representation of the CERN beta-beam layout can be seen in Figure 1.18. The proton driver produces a $2.2 \mathrm{GeV} / \mathrm{c}$ proton beam from the CERN Super Proton Linac (SPL). This is then incident on the ISOL target and ion source which produces both ${ }^{6} \mathrm{He}$ and ${ }^{18} \mathrm{Ne}$. The core of the cylindrical target would comprise a high Z metal, mercury for example, for neutron production. This core is surrounded by an envelope of $\mathrm{BeO}$ which produces ${ }^{6} \mathrm{He}{ }^{9} \mathrm{Be}(n, \alpha)$. The production mechanism for ${ }^{18} \mathrm{Ne}$ is direct proton spallation from a $\mathrm{MgO}$ target. The ion acceleration will initially happen in a linac, which will accelerate to 20-100 MeV/u. Multi-turn injection to a Rapid Cycling Synchrotron would then accelerate to $300 \mathrm{MeV} / \mathrm{u}$. Injection to the CERN PS would follow with 16 bunches, each containing $2.5 \times 10^{12}$ ions. This is reduced to a bunches during acceleration to $\gamma=9$. In the final acceleration stage these bunches are fed to the SPS and accelerated to $\gamma=150$. The decay ring envisaged is a racecourse shaped ring with a $2500 \mathrm{~m}$ straight section aimed toward the detector. This will allow approximately $35 \%$ of the decays to occur in the straight section at this energy.

\subsection{Conclusion}

The discussion in this chapter should present the current state of knowledge of neutrino oscillation physics. A summary of the world best limits on the neutrino mixing parameters can be seen in Figure 1.19. Neutrino oscillations are the first evidence of physics beyond the Standard Model of particle physics and post-LHC will be arguably the focus of the high energy 
fundamental physics community. In the future the emphasis will be on precision measurement of the mixing parameters, specifically the angle $\theta_{13}$ and possible $\mathrm{CP}$ violating phase $\delta$. This could eventually lead to an explanation of the matter-antimatter asymmetry observed in nature. Discussion has been made of the key experiments in building the current knowledge base and we have briefly discussed some possible future facilities, namely Super beams and Beta beam, proposed to measure more accurately the parameters $\theta_{13}$ and $\delta$. The best facility to accurately measure these neutrino mixing parameters ${ }^{2}$, The Neutrino Factory will be detailed in the following chapter.

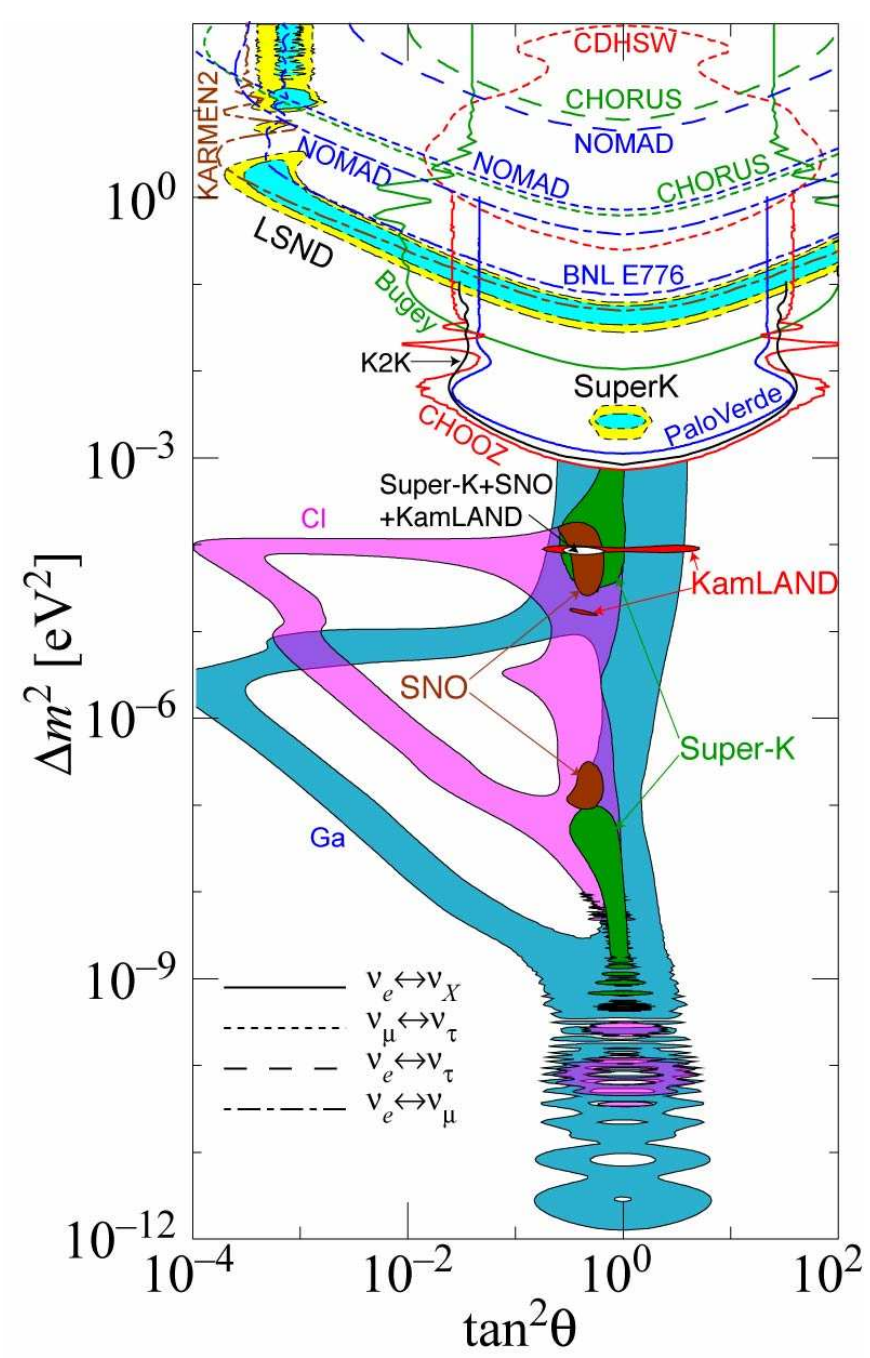

http://hitoshi.berkeley.edu/neutrino

Figure 1.19: Summary of current experimental limits on neutrino oscillation parameters [35]

\footnotetext{
${ }^{2}$ Over the majority of the solution space
} 


\section{Chapter 2}

\section{The Neutrino Factory}

\subsection{Introduction}

The basic concept for what is now termed a Neutrino Factory was first proposed more than 30 years ago [36][37][38]. The first proposal for conceptually the Neutrino Factory that one still discusses was first proposed in 1997 by Geer [39]. This idea was based on the creation of muons from an intense, low energy pion source. To increase the intensity of the muon beam the muon phase-space is compressed and the beam is then accelerated to the required energy. These muons are then injected into a storage ring with straight decay section pointing to neutrino detectors.

\subsection{Neutrino Factory Design}

\subsubsection{Proton Driver}

The main requirement of the proton driver is the production of the baseline $\mu^{ \pm}$decays per year for 10 years for each charge conjugate. The efficiency of the muon front-end and acceleration also plays a very important part in providing this decay flux and will be discussed later. The design of such a proton driver requires a large $R \& D$ investment as the beam power required to achieve this rate of muon production is around $4 \mathrm{MW}$. To put this in perspective, in the short term, new accelerators are planned that achieve 0.75MW (JPARC, Japan) and 1.4 MW (SNS, US) so the increase in intensity will be a great challenge. 


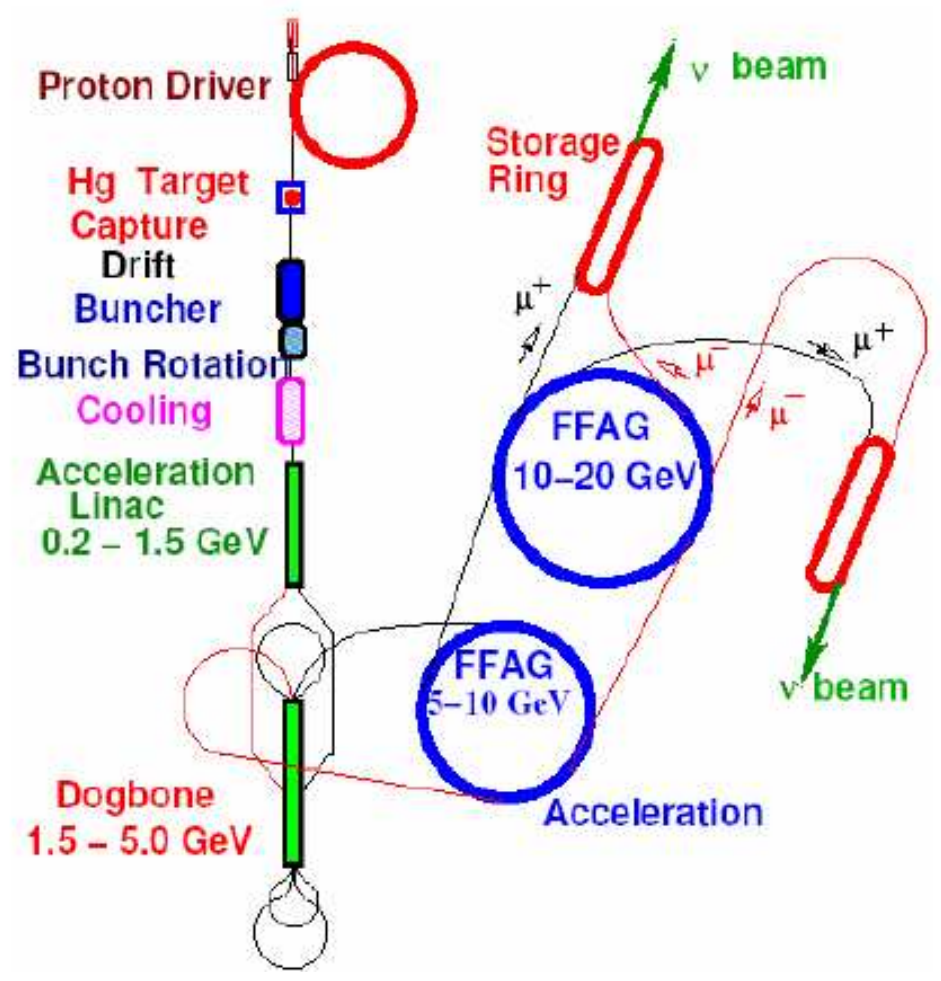

Figure 2.1: Baseline Neutrino Factory design from The International Scoping Study.

\subsubsection{Target and Pion Capture}

The pion production and capture in a Neutrino Factory will be a great technical challenge. The main problem is the huge energy density created in the target when the proton beam is incident. Critical specifications for the target make it difficult to reduce this energy density: the target dimensions cannot be made larger transversely as this would lead to significant decrease in pion production due to re-interactions within the target; also the large power deposition must be dumped very quickly in short pulses to facilitate easy reduction in muon energy spread via phase rotation. The thermal shock produced by this energy deposition can pose severe problems for solid targets. There are currently two proposed schemes for a Neutrino Factory target. In the UK, solid rotating metal targets are being studied. In Europe and the US, R\&D into liquid mercury jet targets is currently being pursued, notably the MERIT experiment [40]. The details of these schemes will be detailed more thoroughly in Chapter 3 especially the solid UK target where a study of pion production as a function of proton driver energy for different hadronic production engines and comparison of these studies with the only current experimental data in that energy regime. Pion collection from the target has been proposed to be achieved through 
one of two concepts. The use of high-field solenoid or a magnetic horn design. The advantage of the use of a 15-20 T solenoid is that both muon charges can be captured. This is obviously only an advantage if the subsequent accelerator facility is designed to transport both $\mu^{ \pm}$

\subsubsection{Muon Phase Rotation and Cooling}

The muon front-end transports and modifies the beam from the beginning of the pion decay channel until injection into the acceleration phase. Pion-Muon decay kinematics result in the bunch length from the decayed muons being short but having a large momentum spread of between $100 \rightarrow 300 \mathrm{MeV} / \mathrm{c}$. This momentum spread is a problem for the muon accelerators and must be reduced. The first method used will be phase rotation. This technique involves using RF to speed up the slower muons and slow down the quicker ones. This results in a long bunch length but one with a very dense core in energy narrowly centred around $100 \mathrm{MeV} / \mathrm{c}^{2}$. The current favoured design adopted by two major studies [41][42] involves the use of two induction linacs surrounding a drift space.

The muons produced from the pion decay after the target occupy a phase space volume too large to inject into a conventional accelerator. The solution to this problem is to reduce the transverse emittance of the beam. Familiar methods of cooling such as stochastic, electron and laser cooling are not feasible when it comes to the design of a Neutrino Factory. This is because for effective cooling these methods take of the order a few seconds to minutes, this is too slow to cool muons which decay in $2.2 \mu s$ on average.

To achieve this essential transverse cooling for a Neutrino Factory we must take a novel approach, Muon Ionisation Cooling. As the name suggests muon ionisation cooling is cooling due to ionisation losses in material. As the muon traverses the matter it loses energy, its emittance is reduced in both the transverse and longitudinal components. Ionisation in the material decreases muon momentum while, to first order, keeping the beam size constant. The reduction in energy of the muons is obviously not desirable and this energy must be replaced. This is done by the RF cavities, which accelerate the muons and replace the longitudinal component of momentum. This will be discussed in more depth later when discussing the MICE experiment. 


\subsubsection{Muon Acceleration}

The muons exiting the front-end of a Neutrino Factory must then be accelerated before injection into decay rings. This acceleration must be very prompt as the muons have a very short lifetime of $2.2 \mu \mathrm{s}$. The acceleration technique chosen must be able to supply intense beams of $\approx 10^{21}$ muons in the range $20-50 \mathrm{GeV}$ per year. There are different schemes being considered at the moment based primarily on Recirculating Linear Accelerators (RLA) and Fixed-Field Alternating Gradient (FFAG) accelerators or combinations thereof. The muon lifetime restriction eliminates even fast-cycling synchrotron accelerators due to the time required to cycle the magnets.

The muons are then accelerated using a linac and two Re-Circulating Linacs (RCL) in the scheme mentioned. This design involves the muons being passed through the accelerating sections multiple times. There are different designs for the acceleration section of a Neutrino Factory. Alternative solutions include the use of high-gradient conventional Linacs, RCLs and FFAG rings.

\subsubsection{Storage}

The accelerated muons are captured in a storage ring. Again there are many different designs for this storage ring including the triangular shape, 'racetrack' and 'dogbone' configurations (a double 'racetrack' design is shown in Figure 2.1). The overriding requirements are that the arcs should be minimised and the straight sections should point to the neutrino detectors chosen for that specific Neutrino Factory site.

\subsection{Physics at a Neutrino Factory}

A brief overview of the current status of neutrino physics is that we have a framework in which neutrino oscillations are explained minimally by three mixing angles: one which is large but not maximal; one which is possibly maximal; one which is small but not necessarily zero. Also, again minimally, by two mass splittings one of which is around 30 times larger than the other. One also has the possibility that if the small mixing angle is non-zero there exists a phase in the mixing matrix which induces a CP violating asymmetry. The most promising future facility for studying the intrinsic neutrino parameters is the Neutrino Factory (NUFACT). 
The limitation in the knowledge of these parameters leads quite naturally to require the following aims for a NUFACT:

- Accurate measurement of $\sin ^{2}\left(\theta_{13}\right)$ for relatively large values and large scope for measuring small values of $\sin ^{2}\left(\theta_{13}\right)$.

- Determining the hierarchy for neutrino mass eigenstates.

- Determination of the value of the $\mathrm{CP}$ violating phase $\delta_{C P}$.

The NUFACT has many advantages in the pursuit of these goals. These include extremely low backgrounds e.g. studies have shown that the background level for a wrong signed muon detection would be $<10^{-4}$, accurate measurement of neutrino flux, intense neutrino flux and the ability to produce beams of both $\nu_{\mu}\left(\bar{\nu}_{\mu}\right)$ and $\nu_{e}\left(\bar{\nu}_{e}\right)$ allowing many oscillation channels to be studied simultaneously. There are twelve key channels which will be essential to probe, six of these are shown in Table 2.1 with the other channels corresponding to their charge conjugates.

The key difference between a Neutrino Factory and a Beta-beam or Super-beam is the inclusion of a muon storage ring. The storage ring can be used to store both $\mu^{+}$and $\mu^{-}$. The "Golden Channel" $\left(\nu_{e} \rightarrow \nu_{\mu}\right)$ at a Neutrino Factory will be shown later to be very useful at studying neutrino parameters via the detection of "wrong sign muons" i.e. the detection of muons of the opposite charge to those contained in the storage ring at that time. This is an example of an appearance channel. Other channels of importance are dissapearance channels and $\nu_{\tau}$ channels.

\begin{tabular}{c|c} 
Channel & \\
\hline \hline$\nu_{\mu} \rightarrow \nu_{\mu}$ & disappearance \\
$\nu_{\mu} \rightarrow \nu_{e}$ & appearance "Platinum Channel" \\
$\nu_{\mu} \rightarrow \nu_{\tau}$ & appearance \\
$\bar{\nu}_{e} \rightarrow \bar{\nu}_{e}$ & disappearance \\
$\bar{\nu}_{e} \rightarrow \bar{\nu}_{\mu}$ & appearance "Golden Channel" \\
$\bar{\nu}_{e} \rightarrow \bar{\nu}_{\tau}$ & appearance "Silver Channel"
\end{tabular}

Table 2.1: Oscillation channels for a storage ring populated by negative muons. The charge conjugate oscillations are present when positive muons are also stored in the ring

The $\bar{\nu}_{e} \rightarrow \bar{\nu}_{\mu}$ golden channel probability can be expressed as in equation 2.1. The functions $\mathrm{X}, \mathrm{Y}$ and $\mathrm{Z}$ are all functions of the known parameters. It can be seen from this equation that 
there is a strong correlation between $\theta_{13}$ and $\delta$. To aid subsequent discussion it should be noted that for baselines where $\mathrm{AL} / 2=0(7300-7600 \mathrm{~km})$ all terms reduce to zero except the first term (X). The baseline at which this happens is normally referred to as the "magic baseline" in the literature. This enables very proficient reduction of degenerate solutions.

$$
\begin{array}{r}
\left.P_{(} \nu_{e} \rightarrow \nu_{\mu}\right)_{ \pm}=\mathrm{X} \sin ^{2} \theta_{13}+\left(\mathrm{Y}_{ \pm}^{c} \cos \delta \mp \mathrm{Y}_{ \pm}^{s} \sin \delta\right) \sin 2 \theta_{13}+\mathrm{Z} \\
\mathrm{X}_{ \pm}=\sin ^{2} \theta_{23}\left(\frac{\Delta_{23}}{\bar{B}_{\mp}}\right) \sin ^{2}\left(\frac{\bar{B}_{\mp} L}{2}\right) \\
Y_{ \pm}^{c}=\sin 2 \theta_{23} \sin 2 \theta_{12} \frac{\Delta_{12}}{A} \frac{\Delta_{23}}{\bar{B}_{\mp}} \sin \left(\frac{A L}{2}\right) \sin \left(\frac{\bar{B}_{\mp} L}{2}\right) \cos \left(\frac{\Delta_{23} L}{2}\right) \\
Y_{ \pm}^{s}=\sin 2 \theta_{23} \sin 2 \theta_{12} \frac{\Delta_{12}}{A} \frac{\Delta_{23}}{\bar{B}_{\mp}} \sin \left(\frac{A L}{2}\right) \sin \left(\frac{\bar{B}_{\mp} L}{2}\right) \sin \left(\frac{\Delta_{23} L}{2}\right) \\
Z=\cos ^{2} \theta_{23} \sin ^{2} 2 \theta_{12}\left(\frac{\Delta_{12}}{A}\right)^{2} \sin ^{2}\left(\frac{A L}{2}\right)
\end{array}
$$

where $\Delta_{i j}=\Delta m_{i j}^{2} / 2 E, B_{\mp}=\left|A \pm \Delta_{23}\right|$ and $\mathrm{A}=\sqrt{2} G_{F} N_{e}$ is the matter parameter, with $N_{e}=$ electron number density in matter.

\subsubsection{Degeneracy}

The determination of the intrinsic neutrino parameters by measuring transition probabilities suffers from the problem of degenerate solutions. It is not possible to determine neutrino oscillation parameters with a neutrino facility with a fixed baseline and fixed energy. The first degeneracy which exists is the intrinsic $\left(\theta_{13}, \delta_{C P}\right)$ degeneracy with $\left(\theta_{13}, \delta_{C P}\right) \rightarrow\left(\bar{\theta}_{13}, \bar{\delta}_{C P}\right)$. There exists a continuous number of solutions that satisfy equation (2.6).

$$
P_{\alpha \beta}\left(\bar{\theta}_{13}, \bar{\delta}\right)=P_{\alpha \beta}\left(\theta_{13}, \delta\right)
$$

The same is true for anti-neutrinos. A complex which could study both neutrino and antineutrino oscillations could eliminate almost all of the continuous degeneracy however Figure 2.2 shows that some ambiguity still remains. The true value and one other solution known as the intrinsic clone. 

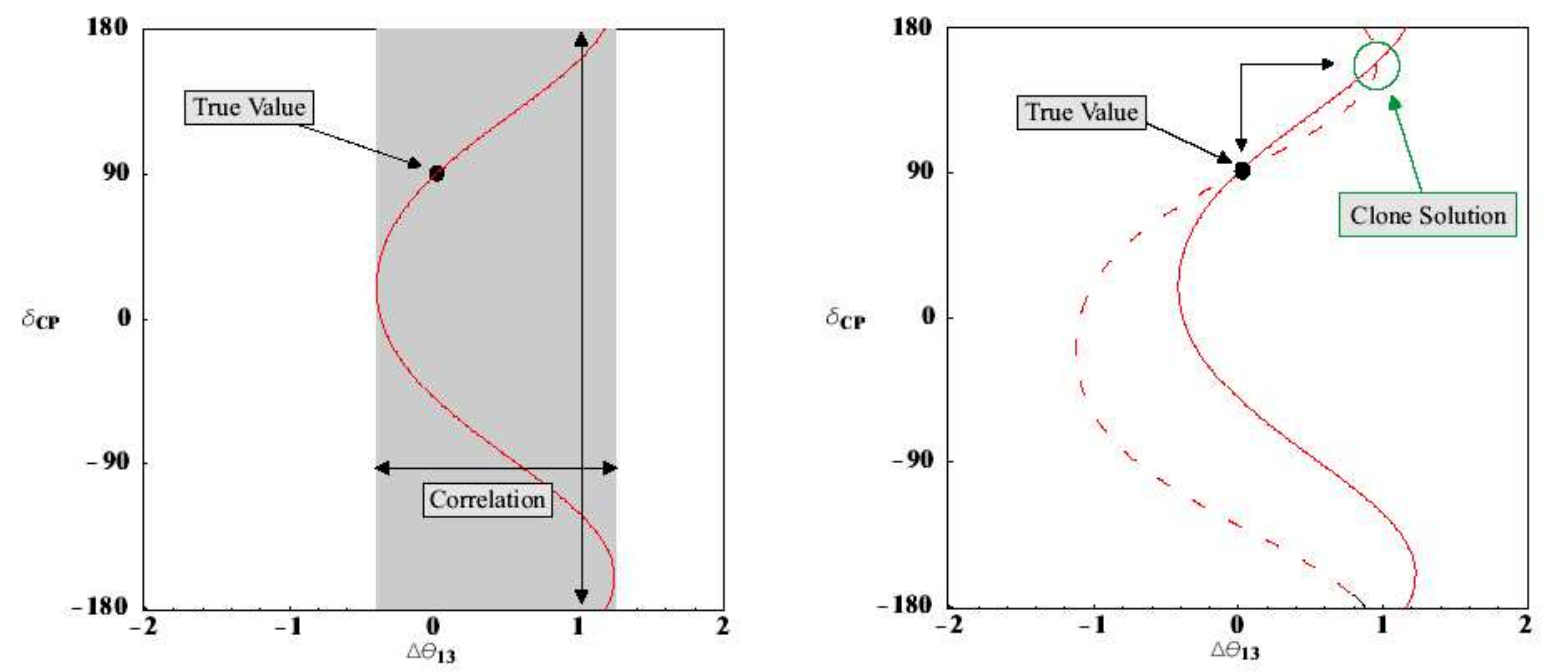

Figure 2.2: Degenerate solutions of $\theta_{13}$ and $\delta$ for one neutrino charge conjugate (left). Degenerate solutions of $\theta_{13}$ and $\delta$ for neutrinos (solid line) and anti-neutrinos(dashed line) showing the remaining intrinsic clone degenerate solution(left)[43].

Generally one can solve this problem in two ways. The first solution is to obtain the probabilities of oscillation for the same channel (i.e. Golden channel) determined at two values of L/E as shown in Figure 2.3. Such a solution could be achieved by considering a Neutrino Factory with measurements at two different baselines. The other scenario which would solve the intrinsic clone problem would be to fix L/E and observe two different oscillation channels (i.e. silver and golden) again this is shown in Figure 2.3.

This, unfortunately, does not completely unfold the degenerate solution space. Two remaining sources of ambiguity exist. The lack of knowledge of the mass hierarchy $\Delta m_{31}^{2} \rightarrow-\Delta m_{31}^{2}$ i.e. the sign of the atmospheric mass squared difference $\operatorname{sign}\left(\Delta m_{23}^{2}\right)=\operatorname{sign}$ atm and the ignorance of the octant value for $\theta_{23}=\operatorname{sign}_{\text {oct }}$ with $\theta_{23} \rightarrow \pi / 2-\theta_{23}$. The sources of these ambiguities are that the leading terms in the atmospheric neutrino oscillation probability $\left(\nu_{\mu} \rightarrow \nu_{\tau}\right)$ depend quadratically on $\Delta m^{2}$ and the probabilities for $\nu_{\mu}, \nu_{e}$ disappearance and $\nu_{\mu} \rightarrow \nu_{\tau}$ appearance depends on $\sin ^{2} 2 \theta_{23}$ and as a result there is still uncertainty as to whether $\theta_{23}$ is greater or smaller than $45 \%$. This leads to an eightfold-degeneracy problem and can be expressed as an extension to equation (2.6) given by equation (2.7). It is essential that a Neutrino Factory complex is able to resolve this degeneracy and as such requires more than one baseline and the ability to study a number of different oscillation channels. The optimisation of baseline will be discussed briefly later. 


$$
P_{\alpha \beta}^{ \pm}\left(\bar{\theta}_{13}, \bar{\delta}, \overline{\operatorname{sign}}_{a t m}, \overline{\operatorname{sign}}_{\text {oct }}\right)=P_{\alpha \beta}^{ \pm}\left(\theta_{13}, \delta, \text { sign }_{\text {atm }}, \text { sign }_{\text {oct }}\right)
$$
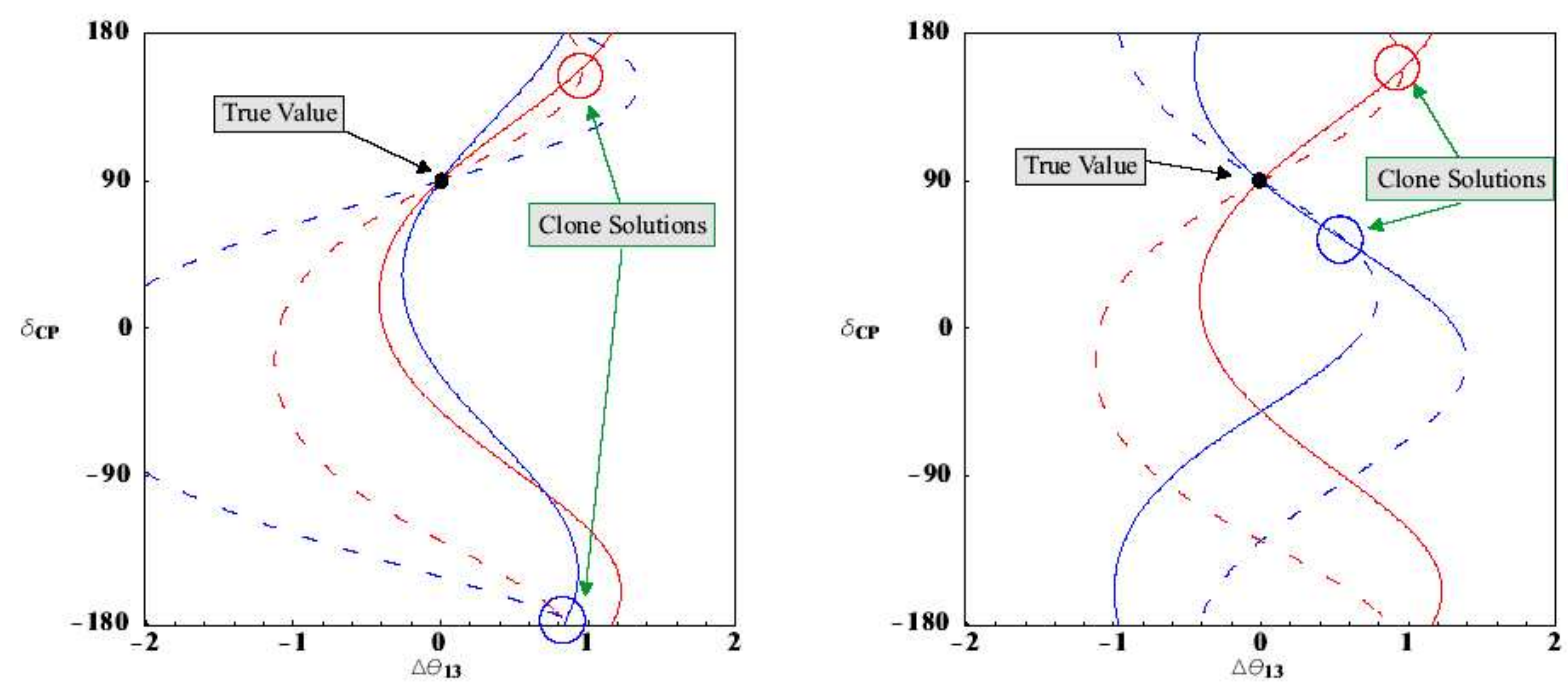

Figure 2.3: Highlighting unique solution by observing same oscillation channel measures at two L/E values (left). Highlighting unique solution by observing silver and golden oscillation channels measured at one value of L/E (right)[43]

\subsubsection{Near Detector}

It is crucial that the neutrino beam is well understood before oscillation effects have time to develop. A neutrino factory near detector will see an extremely large neutrino luminosity of pure neutrino beams. The detector should be placed close to the end of the straight section of the muon storage ring(s). The incident luminosity will be of the order $10^{4}-10^{5}$ times higher luminosities than current accelerators at a detector $50 \mathrm{~m}$ from the storage ring. This is ideal to study some standard neutrino physics like $\sin ^{2} \theta_{W}$, structure functions and neutrino crosssection measurements [44]. In terms of establishing oscillation measurements one requires an accurate flux measurement before the oscillation develops as well as after, the oscillation sets in, at a large oscillation baseline after the oscillation sets into to observe the ratio. One also must understand the backgrounds at a near detector, especially charm production which is one of the most pronounced oscillation backgrounds at the far detector. 


\subsubsection{Optimal performance at a Neutrino Factory}

Detailed studies into the optimal configuration for a Neutrino Factory are currently being undertaken. It is essential to optimise energies, baselines and detector technology to extract the maximum sensitivity to the unknown neutrino parameters. Results from a recent study [45] assume specific detector configuration as detailed below:

- "Golden channel" detection of wrong-sign muons from $\nu_{e} \rightarrow \nu_{\mu}$ oscillations by a magnetised iron detector of 50kT fiducial mass with an energy resolution of $15 \%$ of the incident neutrino energy and MINOS-like performance. It is assumed that the neutrino factory facility can deliver $10^{21}$ muon decays/year for initially 5 years.

- "Silver channel" detection by an Emulsion Cloud Chamber detector of 5kT fiducial mass with an energy resolution of $20 \%$ of the incident neutrino energy

- "Platinum channel" detection of $\nu_{e}$ appearance by a liquid Argon TPC detector of 15kT fiducial mass with an energy resolution of $15 \%$ of the incident neutrino energy

The first performance indicator for a neutrino factory is its $\theta_{13}$ discovery potential. The simulated $\sin ^{2} 2 \theta_{13}$ sensitivity at a neutrino factory as a function of $\mathrm{L}$ and $\mathrm{E}$ by successively folding in statistics, systematics, correlations and degeneracies can be seen in Figure 2.4. When correlation and degeneracy effects are included the importance of the "magic baseline" discussed earlier becomes apparent. This happens at a baseline of approximately $7600 \mathrm{~km}$ and at this distance the intrinsic degeneracy is solved naturally.

The second important performance indicator for a neutrino factory is the CP-discovery potential. As previously discussed the shorter baseline is the one which will give sensitivity to this parameter as the magic baseline has no sensitivity by design. Figure 2.5 shows, for two different choices of $\delta_{C P}$, the sensitivity in determining maximal $\mathrm{CP}$ violation expressed as the reach in $\sin ^{2} 2 \theta_{13}$. It can be seen that for $\delta_{C P}=\pi / 2$ the best performance is associated with baselines of $3000-5000 \mathrm{~km}$ with a very loose dependence on muon energy. It can be seen that for $\delta_{C P}=3 \pi / 2$ the neutrino factory is very restricted in the values of $\sin ^{2} 2 \theta_{13}$ for which CP violation can be determined

The final performance indicator is the mass-hierarchy sign determination sensitivity. Figure 2.6 expresses the mass hierarchy determination sensitivity again in terms of $\sin ^{2} 2 \theta_{13}$ scope. It 

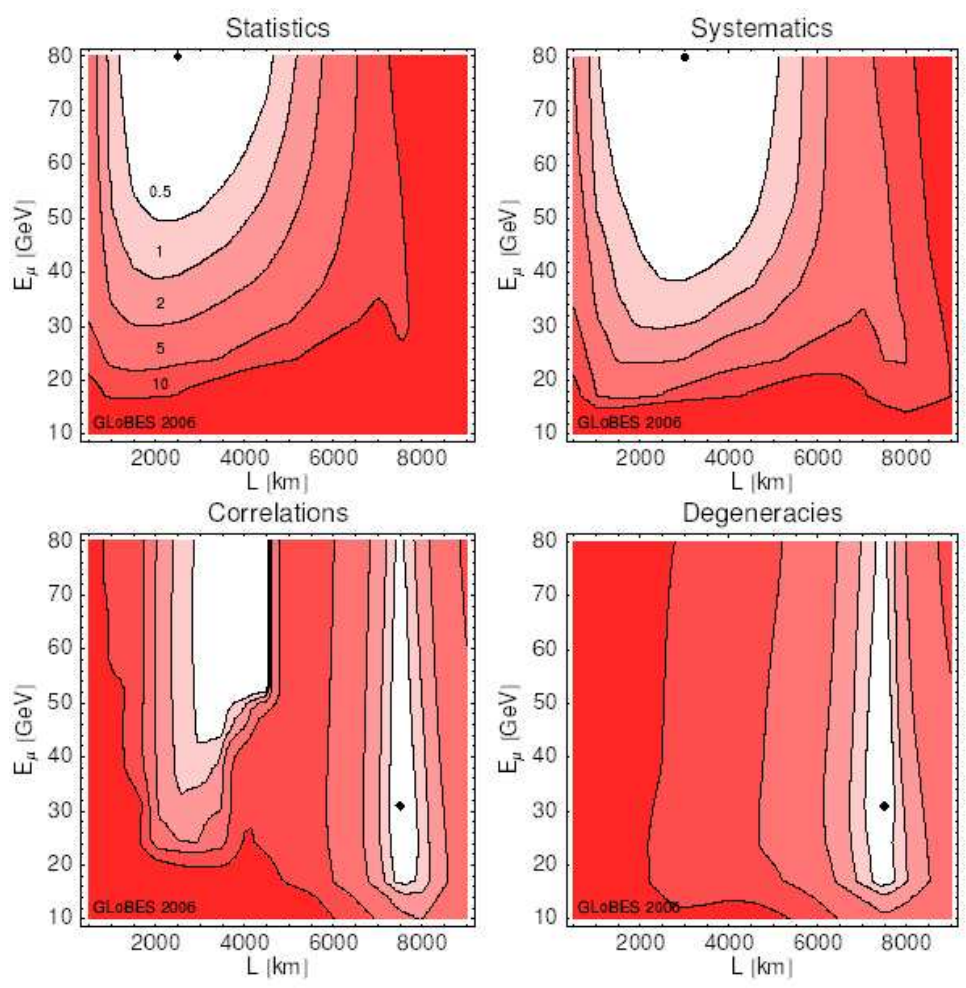

Figure 2.4: The relative sensitivity to $\sin ^{2} 2 \theta_{13}$ compared to the optimal (white). The contours represent regions with a factor of $0.5,1,2,5,10$ above the optimal sensitivity. Each plot is produced by taking into account statistics, systematics, correlations and degeneracies. The optimal sensitivities are $\sin ^{2} 2 \theta_{13}<1.4 \times 10^{-5}$ (statistics), $2.8 \times 10^{-5}$ (systematics), $2.4 \times$ $10^{-4}$ (correlations), $5.0 \times 10^{-4}$ (degeneracies) obtained at the energy and baselines displayed [45].
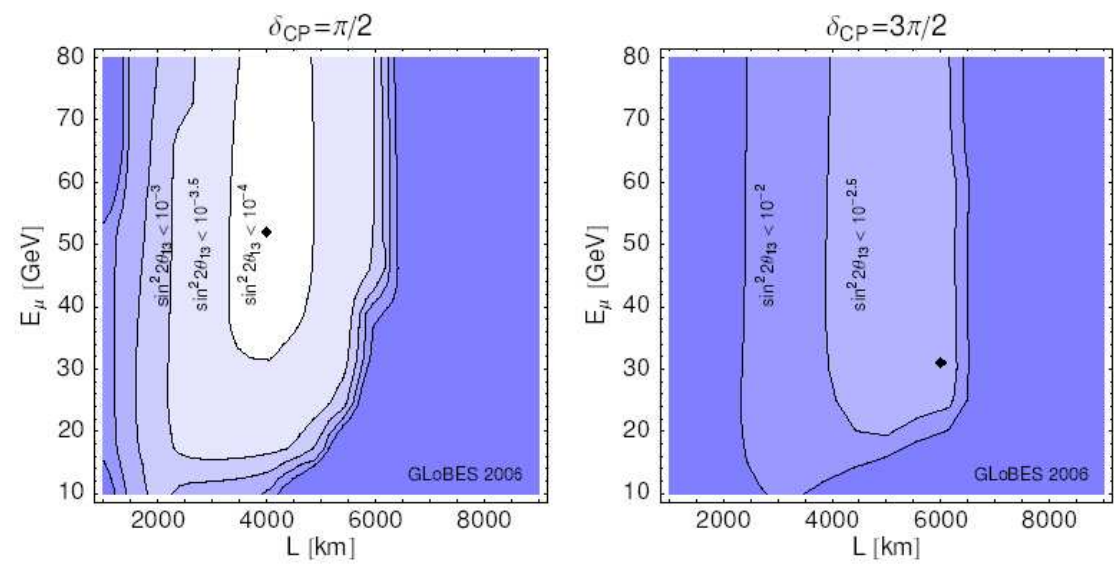

Figure 2.5: Maximal CP-violation sensitivity expressed as the reach in $\sin ^{2} 2 \theta_{13}$ at $\delta_{C P}=$ $\pi / 2$ and $3 \pi / 2$ for different $\mathrm{L}$ and E. $\sin ^{2} 2 \theta_{13}$ reach is given at the $3 \sigma$ CL including correlations and degeneracies. The diamonds mark the minima which are chosen to be $\sin ^{2} 2 \theta_{13}=8.8 \times 10^{-5}$ for left panel and $\sin ^{2} 2 \theta_{13}=1.3 \times 10^{-3}$ for right panel [45]. 
can be seen that muon energy is not of much importance as long as it is greater that $20 \mathrm{GeV}$ and that if $\sin ^{2} 2 \theta_{13}$ is small a long baseline will be essential. The magic baseline looks to be broadly acceptable and seems to be useful once more.
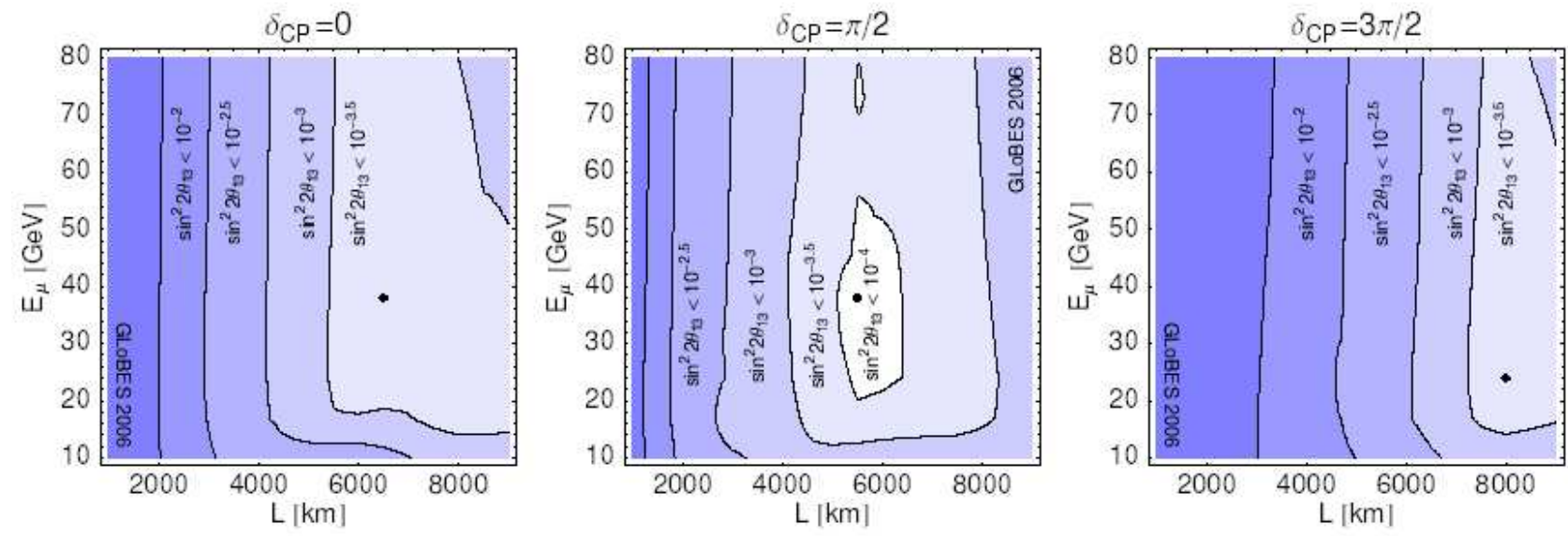

Figure 2.6: Plots show the sensitivity to a normal mass hierarchy for different values of the $\mathrm{CPV}$ phase $\delta_{C P}$ as a function of $\mathrm{L}$ and $\mathrm{E}$. The absolute reach in $\sin ^{2} 2 \theta_{13}$ at $3 \sigma$ including correlations and degeneracies is used as a figure of merit. The minima are at from left to right $\sin ^{2} 2 \theta_{13}=1.8 \times 10^{-4}, \sin ^{2} 2 \theta_{13}=6.7 \times 10^{-5}$ and $\sin ^{2} 2 \theta_{13}=1.6 \times 10^{-4}$ respectively [45].

A problem one has with golden channel measurements at a neutrino factory is that there will be very few wrong-sign muon events seen in the first energy bin (below $10 \mathrm{GeV}$ ) as can be seen in Figure 2.7. Assuming the decay of $50 \mathrm{GeV}$ muons, this energy regime is precisely the location of the first oscillation peak. It is well known that detailed knowledge of the oscillation behaviour on either side of this peak is a very good degeneracy solver and this is exactly the reason why Neutrino Factory results are significantly hindered by degenerate solutions compared with beta-beams and superbeams. The two strategies most commonly discussed to improve are combining different baselines for golden channel oscillations and combining oscillation channels.

A summary of the optimisation discussed [45] is shown in Figure ??. It can be seen that for $\sin ^{2} 2 \theta_{13} \leq 10^{-2}$ a neutrino factory has very impressive sensitivity to both the CP-violation and mass-hierarchy. The figure shows the results obtained from the golden channel and the improvements by sequentially "turning on" in addition a golden channel measurement at the magic baseline with and without an improved detector. The improved detector is discussed more fully in [45] but generally involves a lowering of the detection threshold. The effect of folding in a platinum channel measurement is also shown. It can be seen that uncertainties in the density 

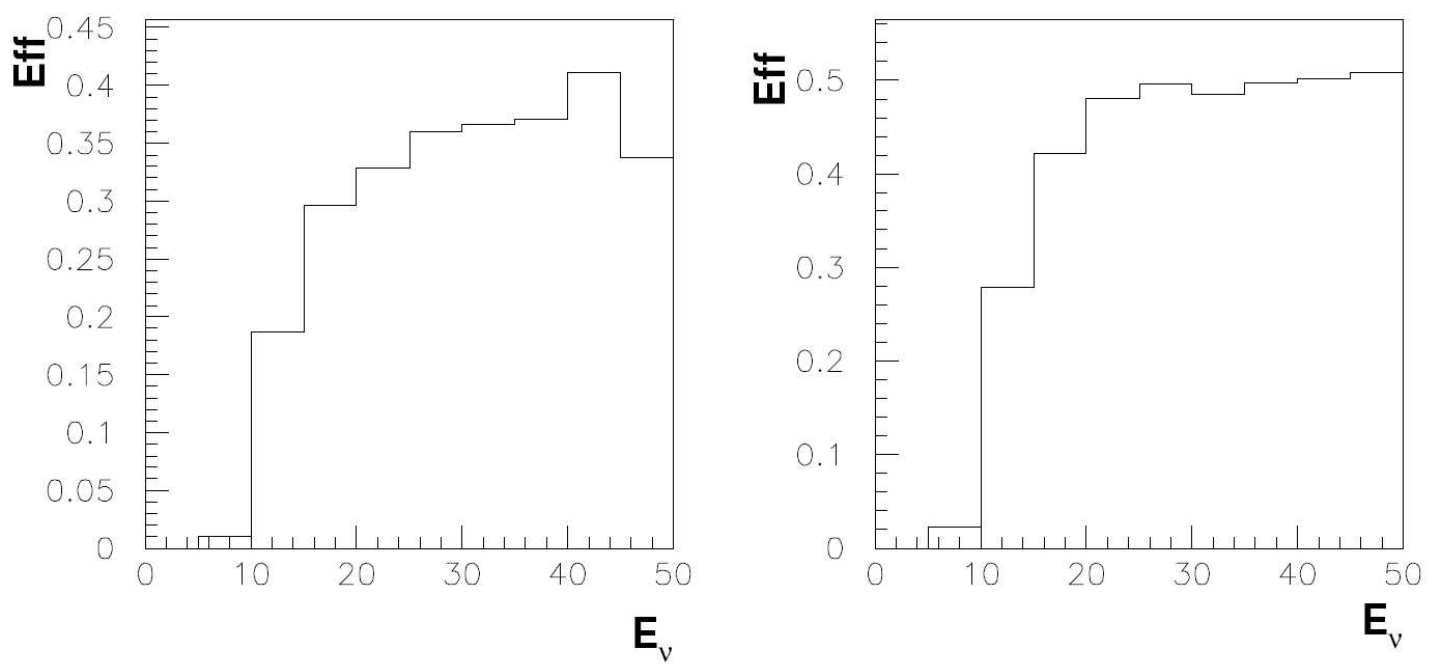

Figure 2.7: Signal efficiencies for $\mu^{+}$(left) and $\mu^{-}$[46] for a golden channel measurement using a magnetised calorimeter as described

of the neutrino propagation medium become important for large values of $\sin ^{2} 2 \theta_{13}$. Generally, it is evident that a neutrino factory with two baselines, one at $\approx 4000 \mathrm{~km}$ and the other at $\approx 7500$ $\mathrm{km}$ will be a very powerful probe for $\sin ^{2} 2 \theta_{13}$, mass-hierarchy and $\delta_{C P}$ determination and that improvements to the far detectors could be very important upgrades.
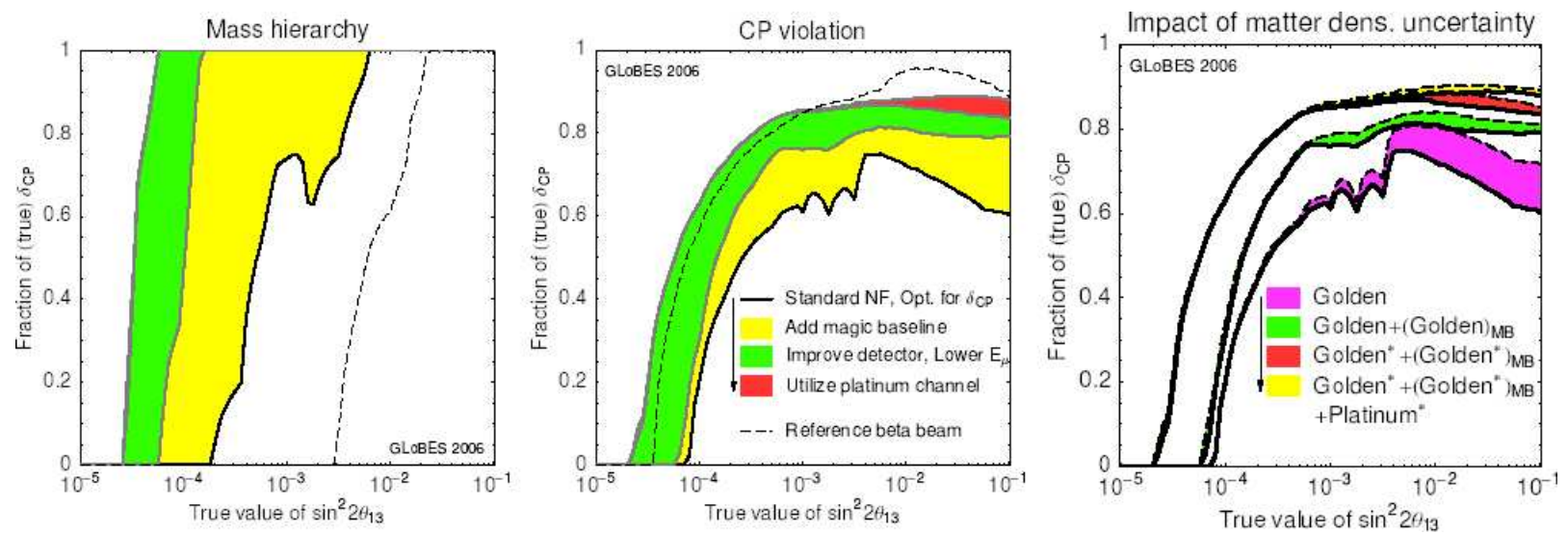

Figure 2.8: Summary of the optimised performance of a neutrino for mass hierarchy (left panel), $\mathrm{CP}$ violation (middle panel) and $\mathrm{CP}$ violation with matter uncertainties included (right panel) at $3 \sigma \mathrm{CL}$. The matter uncertainty is $2 \%$ and the coloured regions are as detailed on the plot. The dashed line is a reference beta-beam[45]. 


\subsection{Conclusion}

This chapter has described in some detail a proposed Neutrino Factory facility. The accelerator facility has been discussed as have studies into the optimal configuration of facility and detector baselines to extract the optimal physics reach. The Neutrino Factory has also been shown to be the optimal oscillation parameter probe over the majority of the $\theta_{13}, \delta$ solution space. 


\section{Chapter 3}

\section{Target Studies at a Neutrino Factory}

\subsection{Introduction}

A viable Neutrino Factory target must be able to produce high intensity pion beams consisting of both charge conjugates. Any target scheme envisaged must be able to withstand extreme physical conditions of temperature and pressure due to the increased proton driver power. Previous experience and $R \& D$ in Neutron Spallation Sources have shown that target solutions for sources with a few MW of beam power are not simple. Schemes to increase the power of current proton driver energies are being developed by most major laboratories with a summary shown in Table 3.1. There are additional complications when trying to apply these constraints to a target suitable for a NUFACT. In a Neutron Spallation source such as the ISIS accelerator, at Rutherford Appleton Laboratory [47] the objective is the production of neutrons. Being neutral they can pass through thick quantities of target material. In a NUFACT we want to maximise pion production and want to minimise reinteractions in the target. This leads to physically smaller target volumes and hence higher power densities which are accompanied by explosive temperatures and pressures.

There are currently two main target schemes being studied. A solid, rotating toroidal heavy metal target which will be studied in this chapter and a liquid mercury jet target. The liquid mercury scheme will be investigated at the nTOFII experiment which is also known as MERIT [40]. The purpose of MERIT is to test the performance of a liquid mercury target in a $15 \mathrm{~T}$ solenoid using a proton beam with pulses comparable to those expected in a $4 \mathrm{MW}$ proton 


\begin{tabular}{|c|c|c|c|c|c|c|c|c|}
\hline Driver & $\begin{array}{l}\text { Power } \\
(\mathrm{MW})\end{array}$ & $\begin{array}{l}\text { Accel. } \\
\text { Scheme }\end{array}$ & $\begin{array}{l}\text { Energy } \\
(\mathrm{GeV} / \mathrm{c})\end{array}$ & $\begin{array}{l}\text { Frequency } \\
(\mathrm{Hz})\end{array}$ & $\begin{array}{l}\text { Protons } \\
\text { per pulse } \\
\left(\mathrm{x} 10^{13}\right)\end{array}$ & $\tau_{p}(\mu s)$ & $\mathrm{N}$ & $\tau_{b}(\mathrm{~ns})$ \\
\hline \multirow[t]{3}{*}{ BNL-AGS } & 1 & Synch & 28 & 2.5 & 9 & 720 & 24 & 3 \\
\hline & 4 & Synch & 28 & 5 & 18 & 720 & 24 & 3 \\
\hline & 4 & Synch & 40 & 5 & 12.5 & 720 & 24 & 3 \\
\hline \multirow[t]{2}{*}{ FNAL } & 2 & Synch & 8 & 15 & 10 & 1.6 & 84 & 1 \\
\hline & 2 & Linac & 8 & 10 & 15 & & & \\
\hline FNAL MI & 2 & Synch & 120 & 0.67 & 15 & 10 & 530 & 2 \\
\hline \multirow{2}{*}{$\begin{array}{l}\text { CERN- } \\
\text { SPL }\end{array}$} & 4 & LAR & 2.2 & 50 & 23 & 3.2 & 140 & 1 \\
\hline & 4 & LAR & 3.5 & 50 & 14 & 1.7 & 68 & 1 \\
\hline J-PARC & 0.75 & Synch & 50 & 0.3 & 31 & 4.6 & 8 & 6 \\
\hline \multirow[t]{4}{*}{ RAL } & 4 & Synch & 5 & 50 & 10 & 1.4 & 4 & 1 \\
\hline & 4 & Synch & $6-8$ & 50 & 8.3 & 1.6 & 6 & 1 \\
\hline & 4 & FFAG & 10 & 50 & 5 & 2.3 & 5 & 1 \\
\hline & 4 & Synch & 15 & 25 & 6.7 & 3.2 & 6 & 1 \\
\hline \multirow{3}{*}{$\begin{array}{l}\text { RAL } \\
/ \text { CERN } \\
\text { KEK } \\
\text { /Kyoto }\end{array}$} & 4 & Synch & 30 & 8.33 & 10 & 3.2 & 8 & 1 \\
\hline & 1 & FFAG & 1 & $10^{4}$ & 0.06 & 0.4 & 10 & 10 \\
\hline & 1 & FFAG & 3 & $3 \times 10^{3}$ & 0.06 & 0.5 & 10 & 10 \\
\hline
\end{tabular}

Table 3.1: Details on envisaged and current proton drivers [48]

driver. The expected number of protons per spill is $2 \times 10^{13}$. In MERIT, a mercury jet of around $1 \mathrm{~cm}$ diameter flowing at $10-20 \mathrm{~m} / \mathrm{sec}$ will interact with an intense $24 \mathrm{GeV}$ proton beam at the CERN neutron Time-of-Flight beamline. The continually flowing mercury jet will cross the proton beam at a small angle of about $40 \mathrm{mrad}$ such that the jet corresponds to roughly two interaction lengths $(30 \mathrm{~cm})$. This target will be placed in a $15 \mathrm{~T}$ solenoid. The studies that will be carried out at MERIT include measurements of how dispersed the jet becomes when "shocked" by the beam during a bunch crossing. Studies of how the magnetic field suppresses this dispersal, investigations on behavior of jets, measurement of various flow rates and beam/jet orientations will be carried out.

The other main scheme for a neutrino factory target is that of a solid continuous or segmented rotating toroidal target. A target of this sort would be robust and provide a relatively simple solution for a neutrino factory. A schematic representation of the target can be seen in Figure 3.1. The toroid in question has dimensions of $1 \mathrm{~cm}$ (radius of circular cross-section) and a rotational radius of around $6 \mathrm{~m}$. The segmented option would use cylinders of target material 


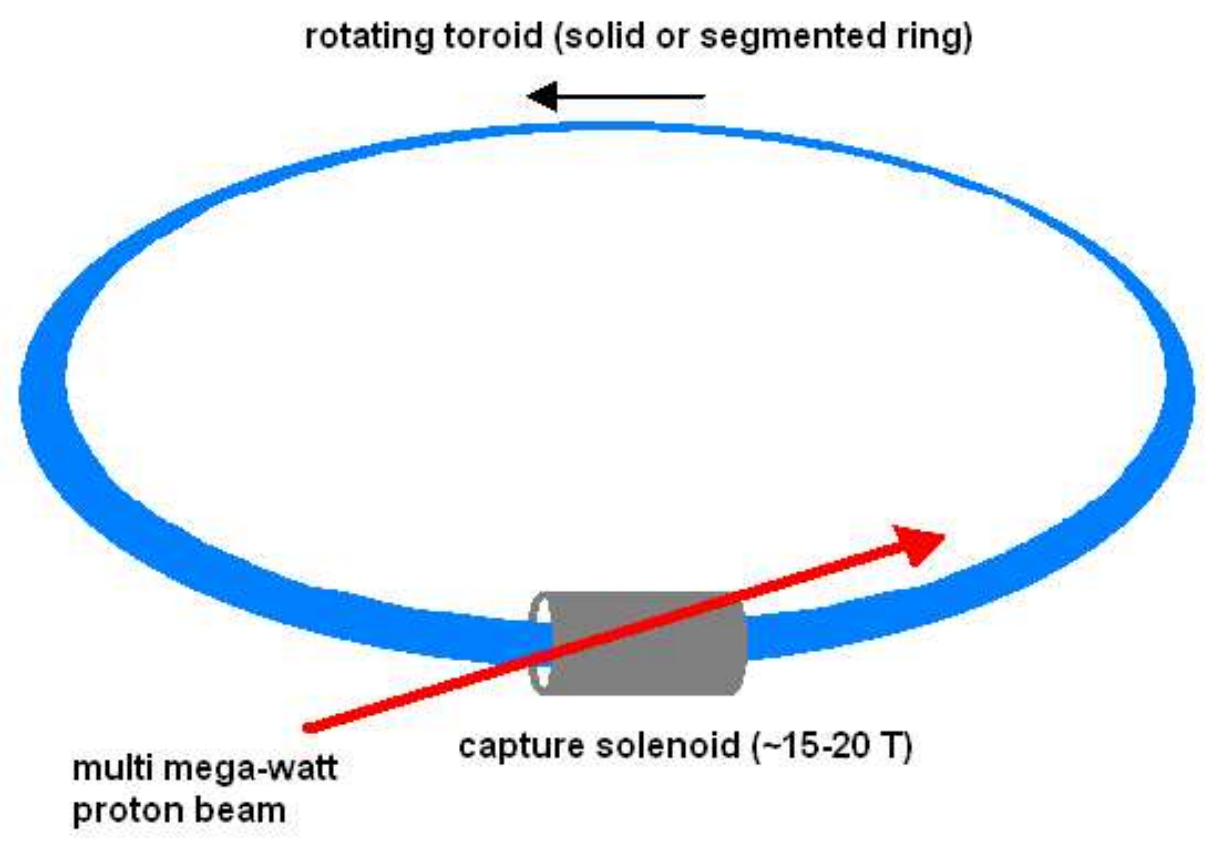

Figure 3.1: Schematic layout of the proposed UK NUFACT target scheme. The target toroid (blue) has been shown as solid but could be segmented in $20 \mathrm{~cm}$ lengths. The dimensions of the toroid are such that it has a $1 \mathrm{~cm}$ radius with the distance between the rotational axis (middle of donut) to torus around $6 \mathrm{~m}$

with dimensions of $1 \mathrm{~cm}$ radius and a length of roughly $20 \mathrm{~cm}$. The region of the toroid which intercepts the proton beam is surrounded by a $20 \mathrm{~T}$ solenoid (or magnetic horn). The proton beam is assumed to have a pulse structure which is $1 \mu \mathrm{s}$ long with one or more 1ns long bunches. It is envisaged that the target will rotate through a $4 \mathrm{MW}$ proton beam at a velocity which is no less that $10 \mathrm{~ms}^{-1}$. The torus will be levitated and rotated by suitable coil geometry. This is preferred to having a rotating spoked wheel supporting a target as this would require the solenoid to be cut to allow passage of the spokes.

The UK design baseline target material is Tantalum. Tantalum is well suited to the task as it is refractory with a high melting point of $3272 \mathrm{~K}$, it has shown to be robust under irradiation by $800 \mathrm{MeV}$ protons from the ISIS accelerator [49] and lastly because it is fairly easy to manipulate. The target will dissipate a mean power of $1 \mathrm{MW}$ assuming an incident $4 \mathrm{MW}$ proton beam. As 
this is not a stopping target this scheme will require a 3MW dump downstream. This region will become very radioactive and must be shielded. This makes the use of a magnetic horn difficult as it is not desirable to dump the beam into the horn itself. The target will rotate in vacuum with the power dumped into it being dissipated to water-cooled vacuum walls. Calculations by Skoro [50] show that at a repetition rate of $50 \mathrm{~Hz}$ the temperature rise per macro-pulse for this target will be $100 \mathrm{~K}$ at an initial target temperature of $2000 \mathrm{~K}$. The major problem with this type of target is that it could suffer significantly from thermal shock [50]. If this is a major problem a slightly modified design can be considered, substituting a solid volume of tantalum with one constructed from an assembly of small (2mm diameter), solid, tantalum spheres cooled by flowing gas or liquid. This reduces the thermal shock experienced. The most important test of these targets are inevitably a suitable in-beam test to determine whether the target has a suitable operational survival lifetime which should be at least one operational year $\left(10^{9}\right.$ pulses $)$

The studies that follow will examine the pion production yields from such a segmented target. Raw pion yield will be investigated as will yields in specific volumes of phase space. The target will be simulated with many different hadronic production engines using GEANT4 [51] and this will be compared to identical simulations using MARS15 [52]. Simulated transmission through two designed neutrino factory front ends will also be detailed as will a study to benchmark both GEANT4 and MARS15 by comparing production cross-sections with those experimentally measured using the HARP experiment [53].

\subsection{GEANT4 simulation and comparison with MARS}

The simulation was carried out using a program called G4Beamline. G4Beamline is a particle tracking program based on the GEANT4 toolkit and FermiLab Beamtools [54]. The most recent version of G4Beamline was used at the time of analysis, which was compiled using Geant4 6.2p02. GEANT4 distributes its physical process models in specific groups called physics usecases. The use-case that was chosen for this simulation was QGSP although it will be shown in this document that its validity for initial proton kinetic energies of $<10 \mathrm{GeV}$ is questionable and other alternatives are investigated. However QGSP is the most accurate physics use-case above 
this energy. The analysis tool used is called Histoscope [55] and is again a Fermilab produced package.

The geometry chosen for this simulation was identical to that chosen in a MARS simulation [56]. In this simulation, a parallel, parabolic proton beam with no longitudinal or momentum spread is incident perpendicular to the upstream edge of a solid tantalum cylinder of dimensions $20 \mathrm{~cm}$ along the beam axis and $1 \mathrm{~cm}$ radius. The tantalum rod was surrounded by a detector volume of vacuum protruding to a distance of $1 \mathrm{~mm}$ around every surface of the rod. The beam was started at exactly the upstream end of the rod. All particles crossing the idealised detector volume were recorded. The constructed geometry can be seen in Figure 3.2.

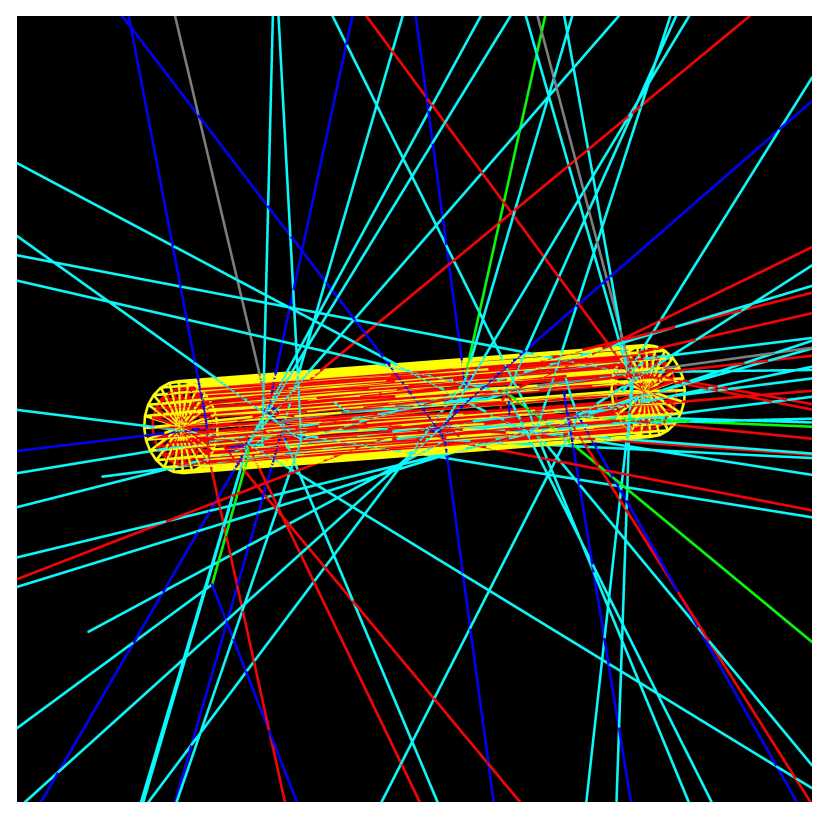

Figure 3.2: Open GL visualisation of the target geometry. The target is coloured red with the virtual detector volume coloured yellow. Protons are red, $\pi^{+}$green, $\pi^{-}$grey, $\mu^{+}$blue and all neutral particles are coloured cyan (almost all are neutrons).

G4beamline accepts four different types of beam: Gaussian, rectangular, ascii and histo. The ascii beam input was chosen for this simulation. Production of the parabolically distributed proton beam was implemented by a $\mathrm{C}++$ program created by the author. This program outputs a beam in the relevant ascii format for G4Beamline to read. The program is fairly flexible and will provide a parabolic beam scaled to any size, with any number of events of any particle of any uniform momentum. This program is available from the author. The parabolic beam has the distribution $f(r) \propto 1-\mathrm{r}^{2}$ with $\mathrm{r}$ the scaled radius of the desired beam. Each Monte Carlo 
run consisted of $100 \mathrm{k}$ protons on target with each job submitted to the Scotgrid tier 2 computer farm to process. Proton beams with kinetic energies in the range $2.2 \mathrm{GeV} \longrightarrow 120 \mathrm{GeV}$ will be studied. This range covers proposed future proton drivers envisaged for a Neutrino Factory (Table 3.2).

\begin{tabular}{|l|l|l|}
\hline $\begin{array}{l}\text { Proton Energy } \\
(\mathrm{GeV})\end{array}$ & $\begin{array}{l}\text { Proton Momen- } \\
\text { tum }(\mathrm{GeV} / \mathrm{c})\end{array}$ & Proton Driver \\
\hline \hline 2.2 & 3.0 & CERN SPL \\
3 & 3.8 & RAL ISIS upgrade low energy ring \\
4 & 4.8 & Possible highest energy for SPL \\
5 & 5.9 & RAL Study for green field site \\
6 & 6.9 & RAL ISIS upgrade high energy ring (6-8GeV) \\
8 & 8.9 & Fermilab driver study 2 \\
10 & 10.9 & RAL study for machine in the CERN ISR tunnel, \\
15 & 15.9 & Fermilab driver study 1 (16GeV) \\
& 20.9 & Brookhaven AGS upgrade (24GeV) \\
20 & 30.9 & JPARC high energy ring initial energy, RAL \\
30 & & study for a CERN PS replacement in the ISR \\
& tunnel \\
40 & 40.9 & JPARC high energy ring ultimate energy \\
50 & 50.9 & \\
75 & 75.9 & 100.9 \\
100 & 120.9 & Fermilab main injector, currently in use for \\
120 & & NuMI beam \\
\hline
\end{tabular}

Table 3.2: Incident proton energies used in simulations [56]

\subsection{Pion yields as a function of incident proton en- ergy}

The first quantity studied is the total number of pions produced by the tantalum target as a function of proton energy. This makes no provision for investigating the pion phase space volume that can be captured by the downstream optics. This will be partially dealt with in later sections. The absolute numbers of $\pi^{+}$and $\pi^{-}$produced are not particularly useful quantities to study when looking for the optimal proton energy for a Neutrino Factory. A more appropriate quantity is pions per proton normalised to the proton beam energy, as this is proportional to number of 
pions produced per Watt of beam power.

The hadronic physics model that is used is QGSP. This is based on a Quark-Gluon String model [51] and is the recommended GEANT4 physics use-case for the majority of the energy range detailed in this document. For energies $<25 \mathrm{GeV}$ the GHEISHA model [51] is used and for $E>25 G e V$ the Quark-Gluon String model is adopted. The quark gluon string model is used for initial projectile-nucleus collisions with cross-sections for string excitations calculated. The behaviour of the nucleus after the initial violent collision is handled by a pre-equilibrium decay model [51].

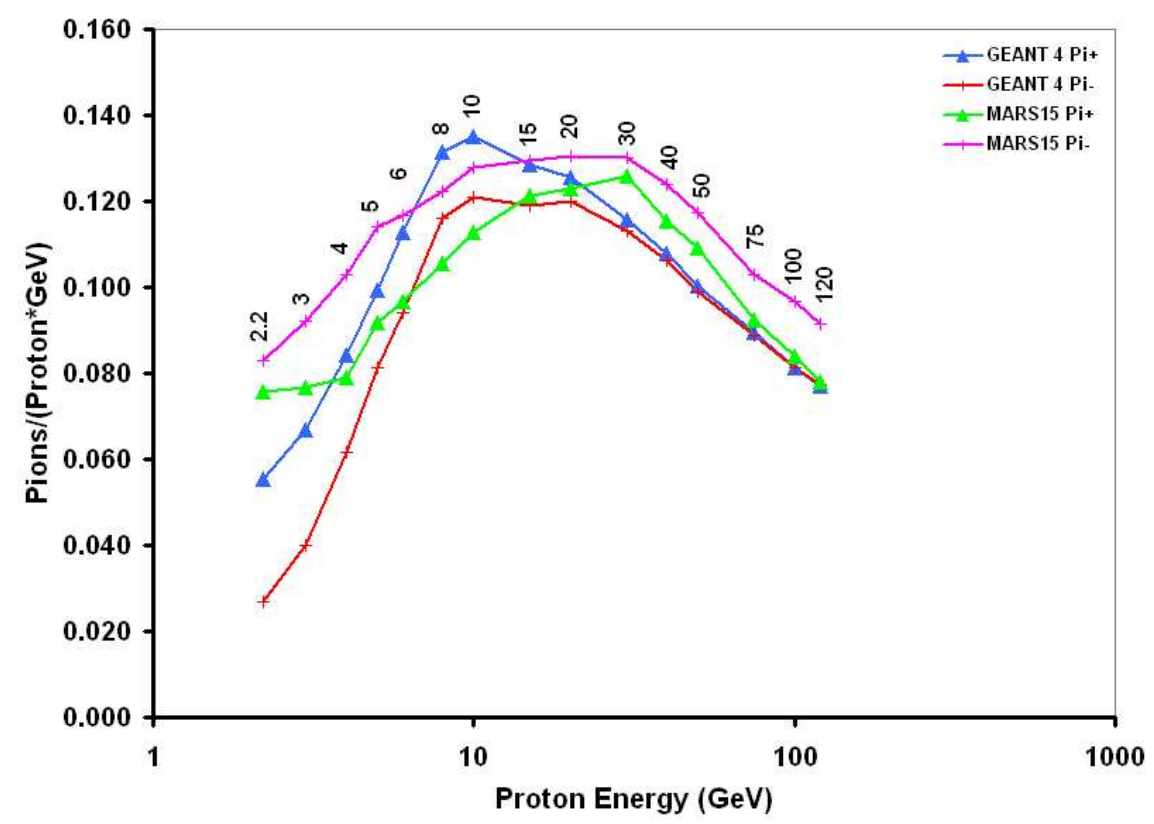

Figure 3.3: Total $\pi^{ \pm}$yields

QGSP was used to produce the results shown in Figure 3.3. Also shown in this plot are the results of a MARS simulation with an identical beam and target geometry $[56]^{1}$. The GEANT4 results clearly show that over the whole range, the number of $\pi^{+}$produced per proton on target is always greater than the number of $\pi^{-}$produced. The MARS data shows the opposite. The variation in discrepancy between the two models can be seen in Figure 3.4. The highest excess of $\pi^{+}$in GEANT4 over MARS is at $8 \mathrm{GeV}$ where there is a $19.6 \%$ difference. The highest excess in $\pi^{+}$in MARS over GEANT4 is at $2.2 \mathrm{GeV}$ where the difference is $36.8 \%$. The difference between models is in the $\pm 8 \%$ range for proton energies of $\geq 15 \mathrm{GeV}$. The disagreement between

\footnotetext{
${ }^{1}$ Note that throughout this document when referring to MARS data its source is [56]
} 
both codes is greater when looking at $\pi^{-}$. For energies of $\leq 6 \mathrm{GeV}$ it is particularly bad with differences of $24 \% \longrightarrow 200 \%$ the later value associated with a proton energy of $2.2 \mathrm{GeV}$. For energies $>6 \mathrm{GeV}$ model agreement is better although still at the $8 \% \longrightarrow 18 \%$ level and the GEANT4 values are consistently below those of MARS.

The results suggest that the optimal proton energy for a neutrino factory is in the range 8-15GeV. It should also be noted that the discrepancy between GEANT4 and MARS decreases as one moves closer to the upper limit of this range.

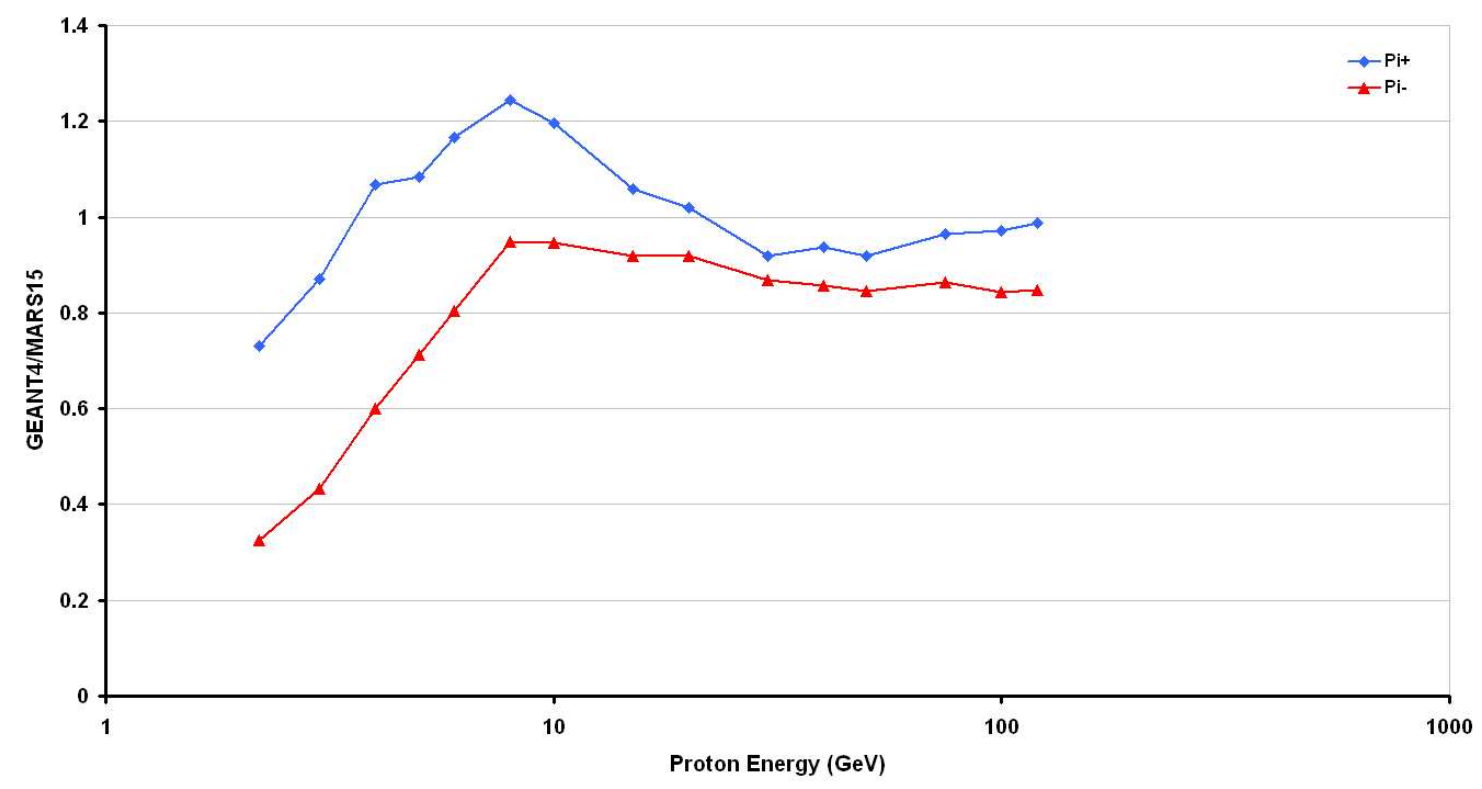

Figure 3.4: Pion per proton*Energy $(\mathrm{GeV})$ variations between GEANT4 and MARS.

\subsection{Comparison between GEANT4 hadronic models}

The region between 1-10 GeV is a grey region in GEANT4 hadronic physics. This energy regime has been probed by the HARP experiment [53] specifically to better understand pion production for a Neutrino Factory. Results from HARP are now starting to be released and will help remedy our poor understanding. It is in this kinematic region that the models describing lower energy hadronic physics e.g. Binary Cascade and Bertini Cascade are at the upper limit of their validity range $(10 \mathrm{MeV} \longrightarrow 8 \mathrm{GeV})$. On the other hand, the quark-gluon string model which deals with higher energy hadronic physics valid in the range $5 \mathrm{GeV} \longrightarrow \mathrm{TeV}$, is at the lower edge of its 
validity. By comparison, the MARS15 code uses two hadron production models. For $\mathrm{E}<5 \mathrm{GeV}$ MARS uses the "Cascade-Exciton Model" CEM2003 algorithms and for energies > 3GeV an "inclusive hadron production" model is used.

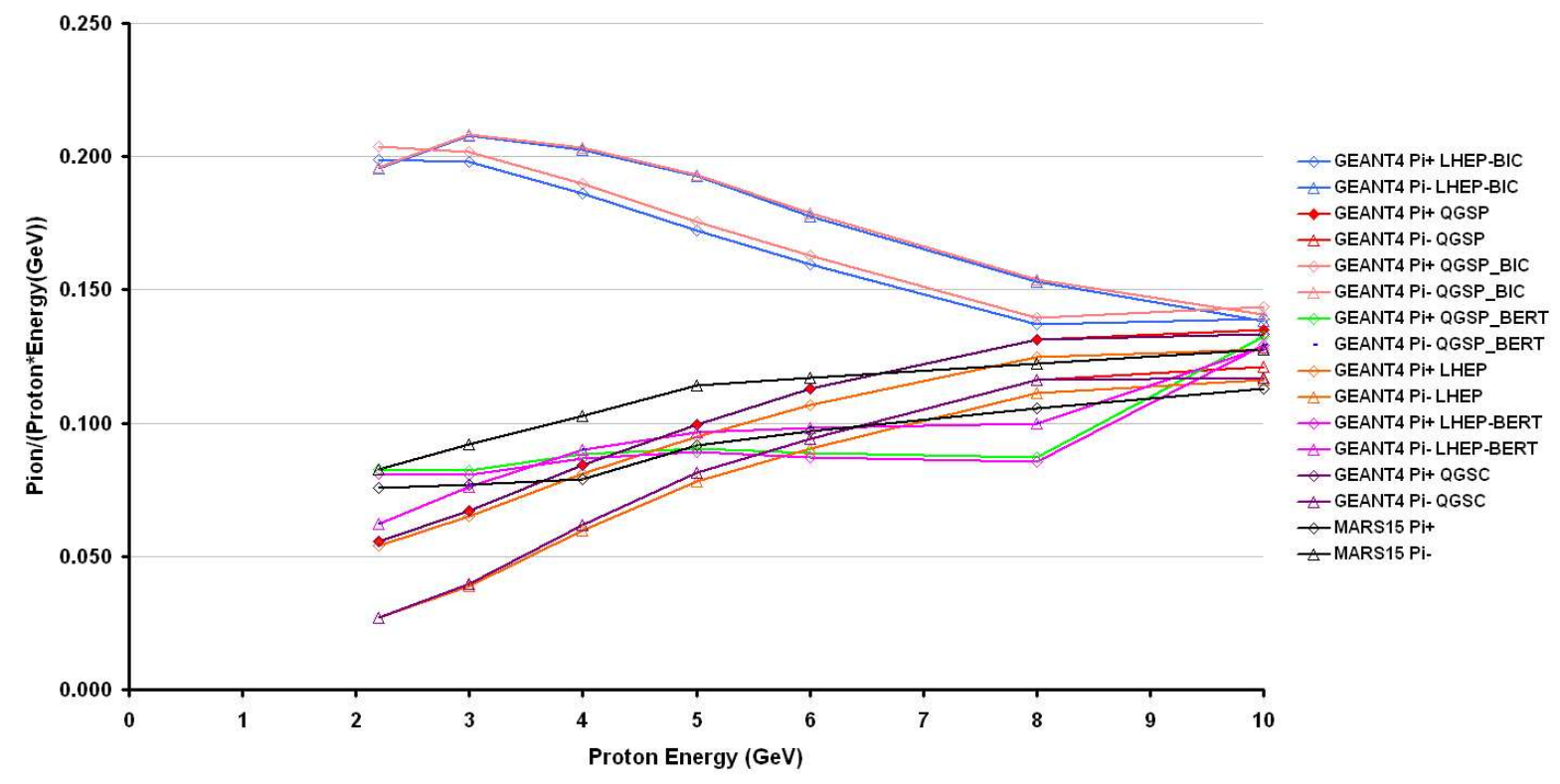

Figure 3.5: Comparison between GEANT4 physics use cases in the range $2.2-10 \mathrm{GeV}$

This uncertainty prompted a study of each GEANT4 physics use-case that has at least part of its range of validity between $1 \longrightarrow 10 \mathrm{GeV}$. The disagreement in $\pi^{ \pm} / \mathrm{GeV}$ yields is considerable and evident in the results shown in the previous section. Further simulations were computed, altering the physics used to calculate the hadron interactions in the target. A summary of the different physics use cases studied and a brief mention of specific physics models used can be seen in Table 3.3.

The results of this comparison are shown in Figure 3.5. It should be noted that certain points are indistinguishable as they lie on top of each other. These include the points for QGSP and QGSC which differ only at 10GeV and LHEP-BERT and QGSP-BERT. The first point to comment on is the distinct variation between models, particularly at low proton energies. The variation between the binary cascade models and others is of the order $100 \%$ at low energies. The ratio of $\pi^{+}$to $\pi^{-}$also varies between models: QGSP, QGSC and LHEP all produce more $\pi^{+}$ than $\pi^{-}$whereas both Binary and Bertini Cascade models produce more $\pi^{-}$than $\pi^{+}$for most of the range. The Bertini models show a large rise between $8-10 \mathrm{GeV}$ where the $\pi^{+} / \pi^{-}$becomes 
$>1$. The agreement between all models including MARS15 is significantly better around the $10 \mathrm{GeV}$ mark.

\begin{tabular}{|l|l|}
\hline GEANT 4 Use Case & Model implemented \\
\hline LHEP & GHEISHA inherited from G3 \\
LHEP-BERT & E $<3 \mathrm{GeV}$ Bertini cascade, E $>3 \mathrm{GeV}$ GHEISHA \\
LHEP-BIC & LHEP-BIC E $<3 \mathrm{GeV}$ Binary cascade, E $>3 \mathrm{GeV}$ GHEISHA \\
QGSP & E $<25 \mathrm{GeV}$ GHEISHA, E $>25 \mathrm{GeV}$ quark-gluon string model \\
QGSP-BERT & QGSP-BERT E $<3 \mathrm{GeV} \mathrm{Bertini} \mathrm{cascade,} 3<\mathrm{E}<25 \mathrm{GeV}$ \\
& GHEISHA, E $>25 \mathrm{GeV}$ quark gluon string model \\
QGSP-BIC & E $<3 \mathrm{GeV}$ Binary cascade, $3<\mathrm{E}<25 \mathrm{GeV} \mathrm{GHEISHA,} \mathrm{E}>25$ \\
& quark gluon string model \\
QGSC & E $<25 \mathrm{GeV}$ GHEISHA, E $>25 \mathrm{GeV}$ quark-gluon string model, \\
& chiral invariance \\
\hline
\end{tabular}

Table 3.3: GEANT 4 Hadronic Physics Summary

\section{Cosine (Off axis angle) (taigetDet)}

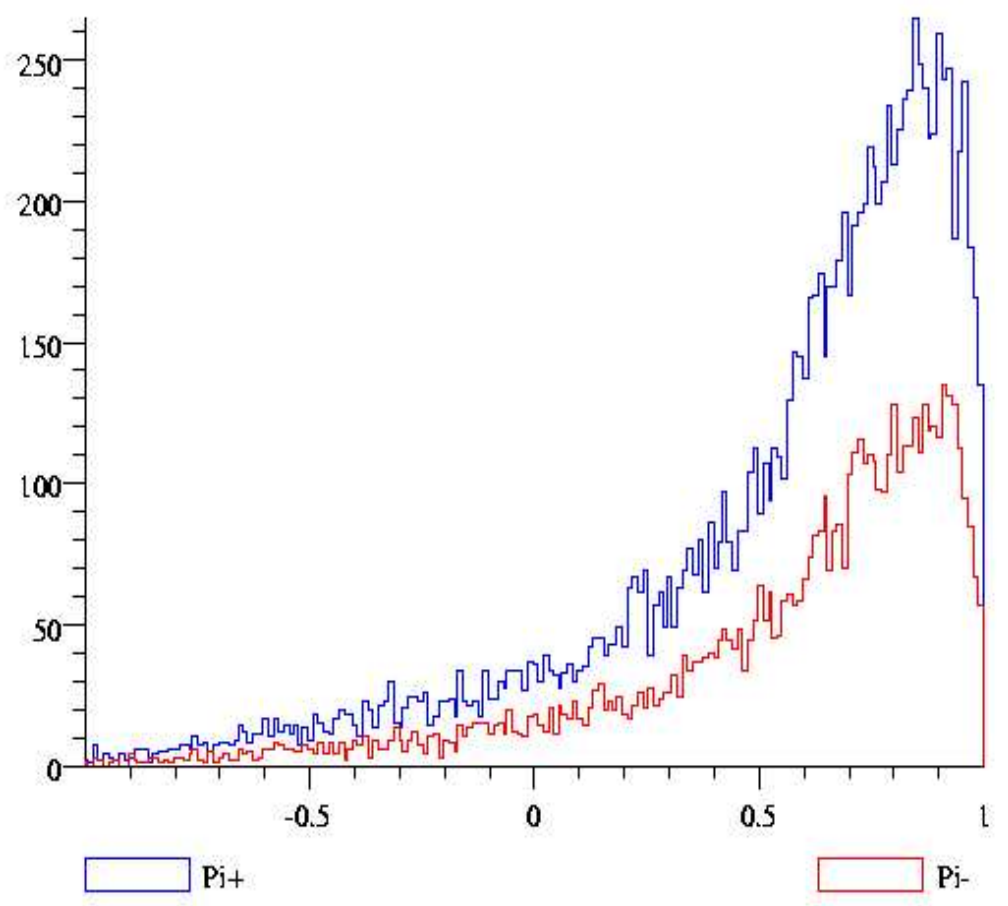

Figure 3.6: Pion angular distribution with incident proton energy of $2.2 \mathrm{GeV}$. X-axis is cosine of the opening angle made between the outgoing pion and the z-axis of the target 


\subsection{Pion angular distribution}

In producing a Neutrino Factory target one is obviously interested in the $\pi^{ \pm}$angular distributions. In this section, the off-axis angle that the outgoing pion makes with the $\mathrm{z}$ axis of the target will be studied. The pion distributions calculated using QGSP created by 2.2 and 15 $\mathrm{GeV}$ incident proton beams can be seen in Figures 3.6 and 3.7 respectively. Both figures have 0.01745 radian bins. It can be seen from close inspection of both plots that outgoing $\pi^{+}$are forward focused to a similar degree when compared with outgoing $\pi^{-}$. It is also evident that as the energy increases the focusing of both $\pi^{ \pm}$increases. This is to be expected. Note that obviously those pions sitting in bins less than $\cos \theta=0$ will be backward travelling and as such are uncapturable as are those with high exit angle (see later).

\section{Cosine (Off axis angle) (targetDet)}

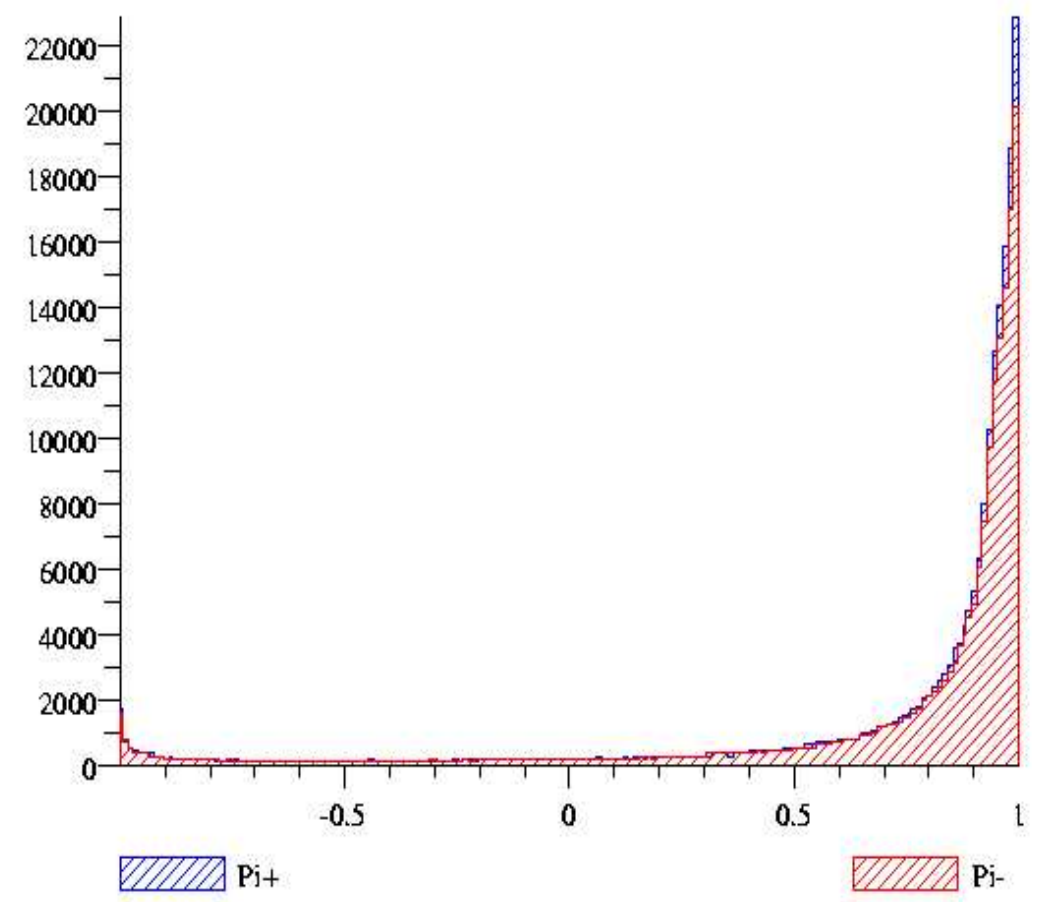

Figure 3.7: Pion angular distribution with incident proton energy of $15 \mathrm{GeV}$. X-axis is the cosine of the opening angle made between the outgoing pion and the $\mathrm{z}$-axis of the target

In Figure 3.8 the pion angular distributions are displayed where the angles that contained $75 \%, 50 \%$ and $25 \%$ of the pions are plotted for both $\pi^{ \pm}$. Also shown are the same values obtained by MARS. Firstly commenting on purely the GEANT4 results it can be seen that generally both $\pi^{ \pm}$are equally focused with differences of $<10 \%$. Also there is a peak in the 
distribution from 20-30 GeV. This is likely to be partially if not wholly artificial as there is a QGSP model transition region at $25 \mathrm{GeV}$. The effect of this is felt more strongly for those pions at highly transverse exit velocities with the effect on the more focused $\pi^{ \pm}$much less. This is understandable as the reaction cross section for pion production is less well known at high transverse angles than those forward focused angles, hence both models will struggle to cope with these particles at the transition region.

Comparing the GEANT4 results with the MARS data it can be seen that in general the $\pi^{ \pm}$are significantly more forward focused in GEANT4. The MARS $\pi^{ \pm}$approach the GEANT4 results for high initial proton energies, in particular in the more forward focused direction. The $\pi^{+}$produced by MARS are closer to the GEANT4 results compared with the MARS $\pi^{-}$.

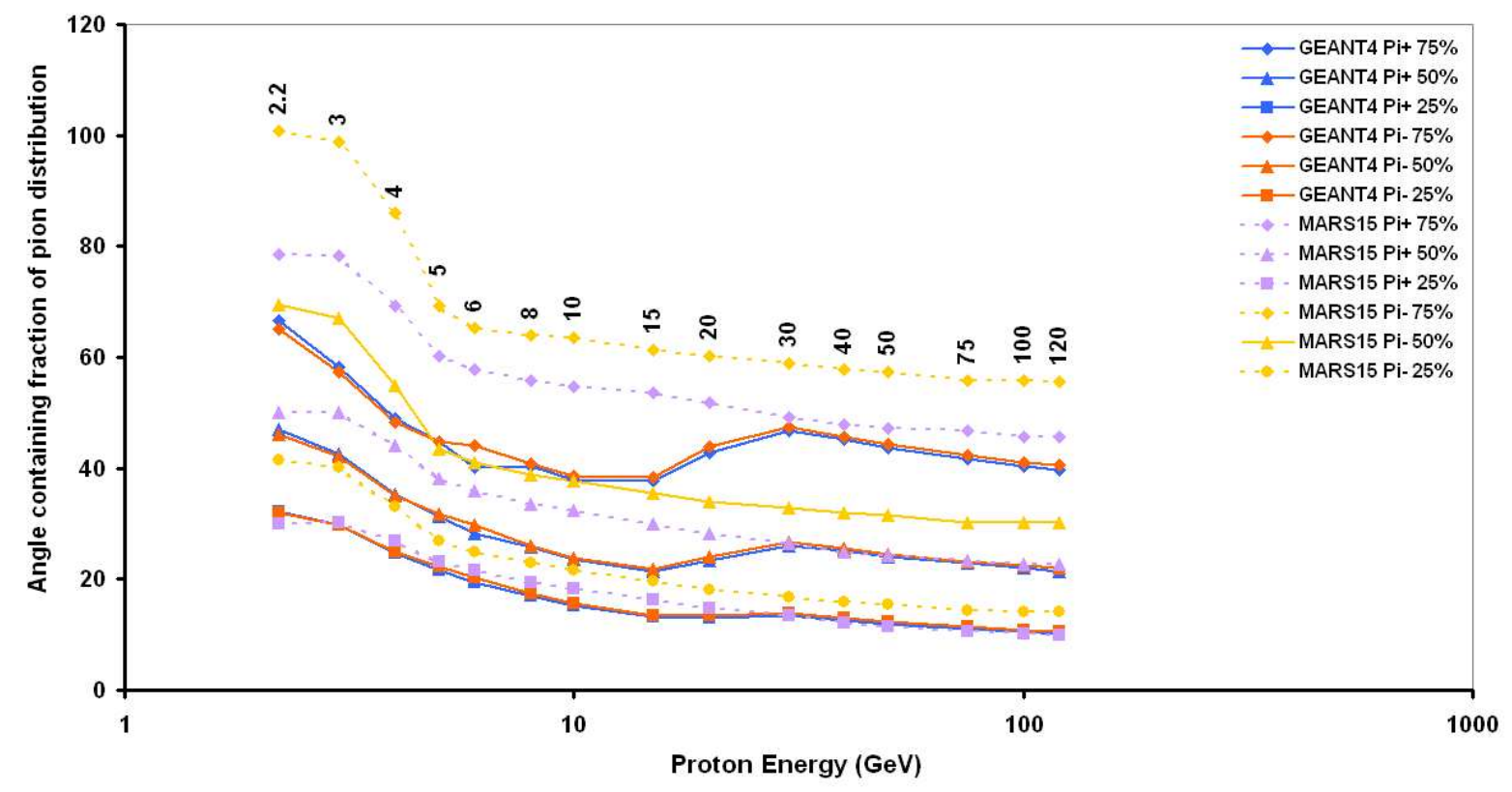

Figure 3.8: Plot of angles (in degrees) containing 25\%, $50 \%$ and $75 \%$ of pion distribution

The final notable feature is that the large dip in the MARS data between $2.2 \mathrm{GeV}$ and $6 \mathrm{GeV}$ does look from these results to be non-physical and a remnant of the MARS model transition in this region. By comparison the GEANT4 results show a more gradual and arguably more believable result. 


\subsection{Pion yields inside $20^{\circ}$ transverse acceptance}

In constructing a target applicable to a Neutrino Factory, obviously the most important aspect of pion production to study is the number of "capturable" pions produced per unit beam power. In an approximate attempt to investigate this, a cut was imposed to the data shown previously. This cut eliminates pions whose exit momentum vector lies within a $20^{\circ}$ cone pointing downstream and centered along the beam axis. Pions lying outside this volume of phase space will be unlikely to be captured by downstream optics.

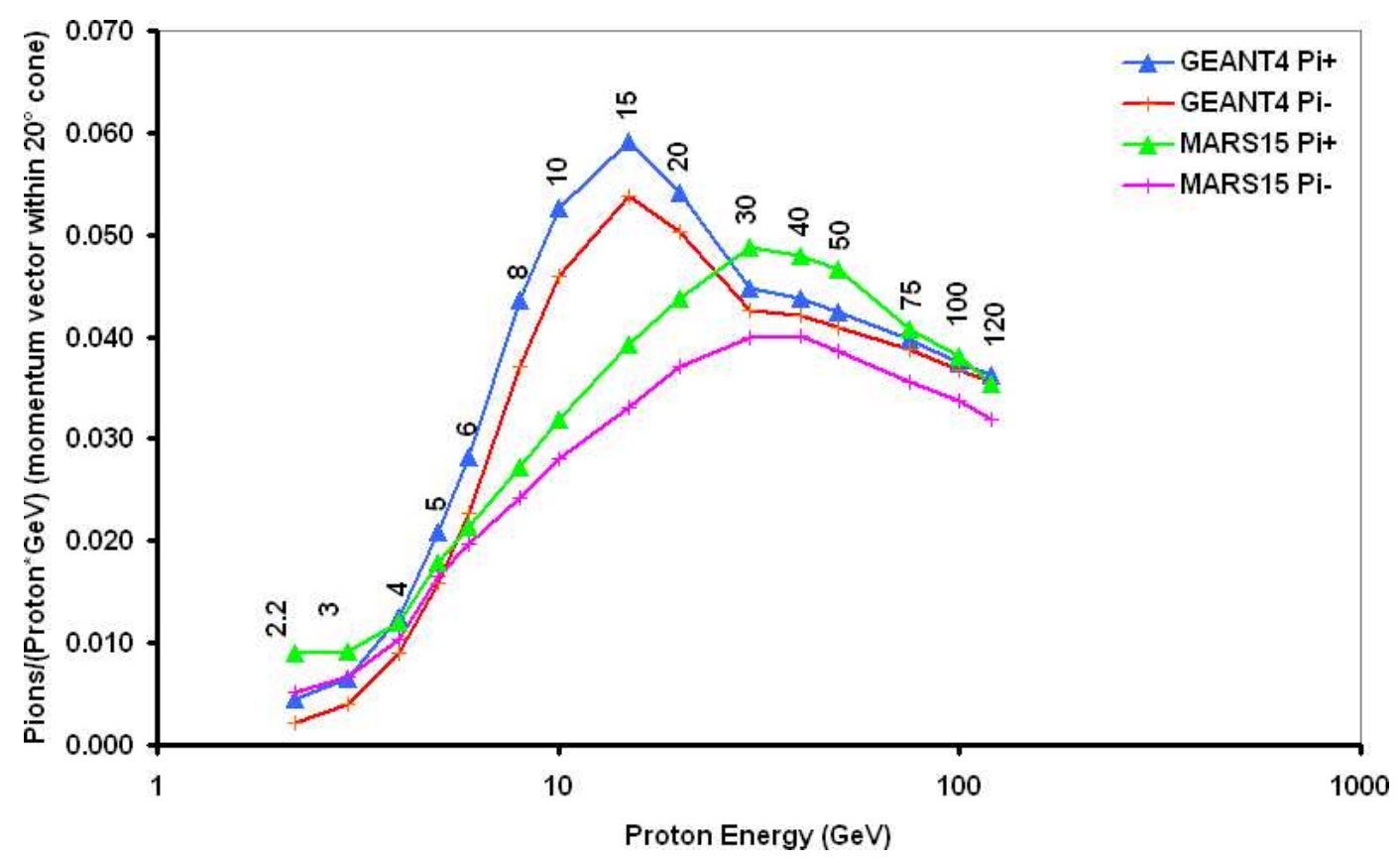

Figure 3.9: Pion per (Proton*Energy $(\mathrm{GeV})$ ) within $20^{\circ}$ cone

The results can be seen in Figure 3.9. The most obvious feature is the peak in the GEANT4 data at $15 \mathrm{GeV}$ for both $\pi^{ \pm}$. The QGSP model transition region at $25 \mathrm{GeV}$ can be clearly seen in the sharp change of behavior from $30 \mathrm{GeV}$ onwards. This peak may not be physical and a further comparison of different physics use-cases should be carried out in the future. The MARS data differs significantly in the peak region with better agreement at lower energies (especially $3-5 \mathrm{GeV}$ ) and higher energies. Pion yields within the $20^{\circ}$ cone are greater in GEANT4 than MARS15 over the range 4-30 GeV although this does include the GEANT4 peak region. It is evident that in this range QGSP calculates pions produced to be more forwardly directed than MARS. Purely based on the GEANT4 results (with a caveat on the model accuracy) a proton 
energy of around $15 \mathrm{GeV}$ looks optimal for a Neutrino Factory.

\subsection{Pion yields within $100 \mathrm{MeV}$ transverse momen- tum acceptance}

In the previous section, an angular cut was imposed to eliminate large numbers of pions that do not have the correct kinematics to be captured by the pion decay channel optics i.e. those pions that are backward traveling or that have highly transverse exit angles. A more effective cut on transverse momentum $(\mathrm{Pt})$ can be made as a population of high momentum pions with high exit angles within the $20^{\circ}$ cone will be moving with a momentum that is too high to be captured. Previous studies [57] have shown that a cut with transverse momentum $(\mathrm{Pt})<100 \mathrm{MeV}$ would be acceptable to study the transmission into the downstream optics. The results of this cut can be seen in Figure 3.10 for the GEANT4(QGSP) and MARS models. The peak mentioned in the previous section is again prevalent as is the model transition region at $25 \mathrm{GeV}$. The peak is more distinct in this plot than in the previous one, the decrease in relative pion yield for high energies accounting for this. The Pt cut has eliminated the fast, highly transverse phase space pions. These pions are uncapturable and are more numerous with increasing incident proton energy. It is interesting to note that the relative pion yields in the range $2.2-20 \mathrm{GeV}$ have remained the same after the additional Pt cut. This implies that these pions are indeed transversely focused. The MARS data shows a similar decrease in yields at higher energies. The final point to note is the almost uniform relative shift in pion yields between GEANT4 and MARS for energies $>30 \mathrm{GeV}$ suggesting that the QGSP algorithms focus pions more in the forward direction than the MARS algorithms. This data (again with the caveat on model accuracy) again suggests the optimal proton energy for a Neutrino Factory is in the range $10-15 \mathrm{GeV}$. 


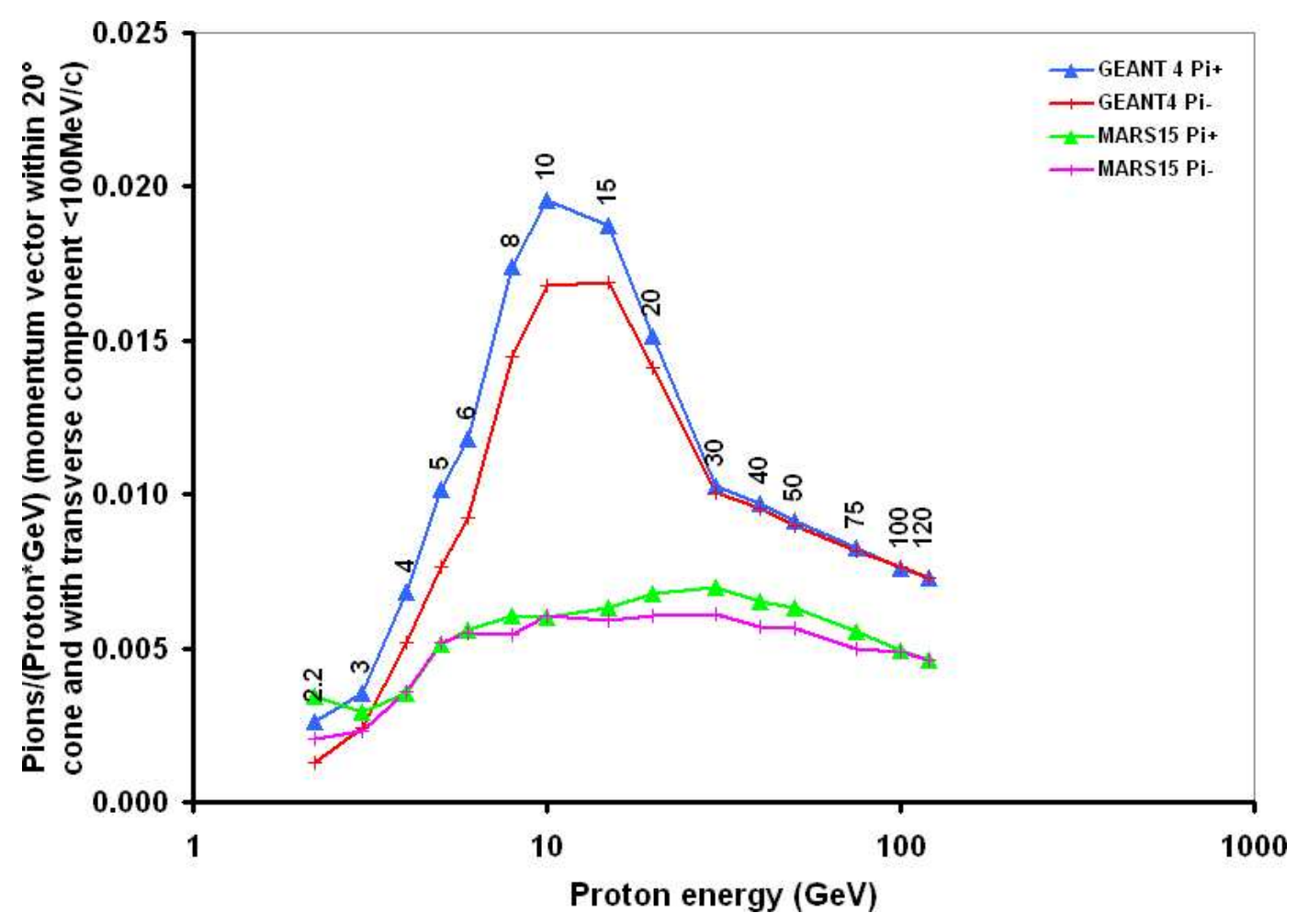

Figure 3.10: Pion per (Proton*Energy $(\mathrm{GeV})$ ) within $20^{\circ}$ cone and $\mathrm{Pt}<100 \mathrm{MeV}$

\subsection{Pion yields in a neutrino factory front-end simu- lation}

The $\pi^{ \pm} \rightarrow \mu^{ \pm}$decay in a neutrino factory happens in the muon front-end of the channel. This section will investigate the pion yields after transmission through two different designs of neutrino factory front-ends, namely the Chicane/Linac and Phase Rotation schemes.

Particles were tracked through both these front ends using a code called Muon1 [57] that in addition to tracking deals with particle decays. The fate of the particles (i.e. whether they made it through the channel and if not where along the channel they were lost) can be seen for the Chicane/Linac(Figure 3.11) and Phase Rotation scheme (Figure 3.13). Both channels were tuned for $2.2 \mathrm{GeV}$. The distributions were sampled for both schemes after the Linac and RF-Cavities for Chicane/Linac and Phase Rotator schemes respectively. The distributions were binned into two dimensional $P_{\text {long }} P_{\text {transv }}$ bins of $30 \mathrm{MeV} / \mathrm{c}$ and weighted into a probability map.

A program was constructed called ProbabilityMap which has the functionality that it could bind with the Histoscope output and calculate weighted transmissions for any particle of any 
momentum in variable bin sizes for any probability map constructed for any channel. This program was then run on the GEANT4 datasets previously produced using the QGSP physics use-case in (see section 3.3) for each of the relevant proton driver energies (Table 3.2). This was repeated for both Chicane/Linac and Phase Rotator schemes and the results can be seen in Figures 3.12, 3.14. This method is very quick and allows easy estimation of transmission along different channnels. Currently only the $2.2 \mathrm{GeV}$ channel has been optimised however it will be very easy to run these datasets using other optimisations when they become available.

\subsubsection{Chicane/Linac pion decay and transport channel}

In the Chicane/Linac scheme the target is surrounded by a $20 \mathrm{~T}$ solenoid followed by a solenoidal decay channel consisting of 32 transversely focusing solenoids. The lack of longitudinal focusing leads to longitudinal bunch length stretching and ultimately a debunched muon beam. Longitudinal compression is then achieved by a reverse-phase-slip achromatic lattice which is referred to here as a bending chicane. The chicane consists of four identical sets of dipole triplets with fields designed such that a pencil poly-chromatic beam will emerge from a triplet as a dispersed beam rotated by $101^{\circ}$. This dispersed beam then enters a second triplet and focused to a pencil beam. This is repeated in the remaining triplets with the bend in the opposite direction. Path-length differences cause recompression longitudinally. The bunched muon beam is then passed through an $88 \mathrm{MHz}$ muon linac which accelerates to $400 \pm 100 \mathrm{MeV}$

The results for the number of pions per unit beam power for the Chicane Linac channel (Figure 3.12) show that more $\pi^{+}$than $\pi^{-}$per proton beam power are transported until $30 \mathrm{GeV}$ where they are transported in equal measures. The peak appears to be around $8 \mathrm{GeV}$ although the dip which appears for both $\pi^{ \pm}$at $6 \mathrm{GeV}$ seems strange and probably non-physical. Negative pions are preferentially transported along the channel at the very highest energies.

\subsubsection{Phase rotation pion decay and transport channel}

The Phase-Rotation scheme involves continuing the solenoid channel before using $31.4 \mathrm{MHz}$ RF-cavities to reduce the energy spread with a goal of $180 \pm 23 \mathrm{MeV}$. This muon beam would then be injected into a cooling ring for reduction of normalised emittance.

The results for the number of pions per unit beam power for the Chicane Linac channel 


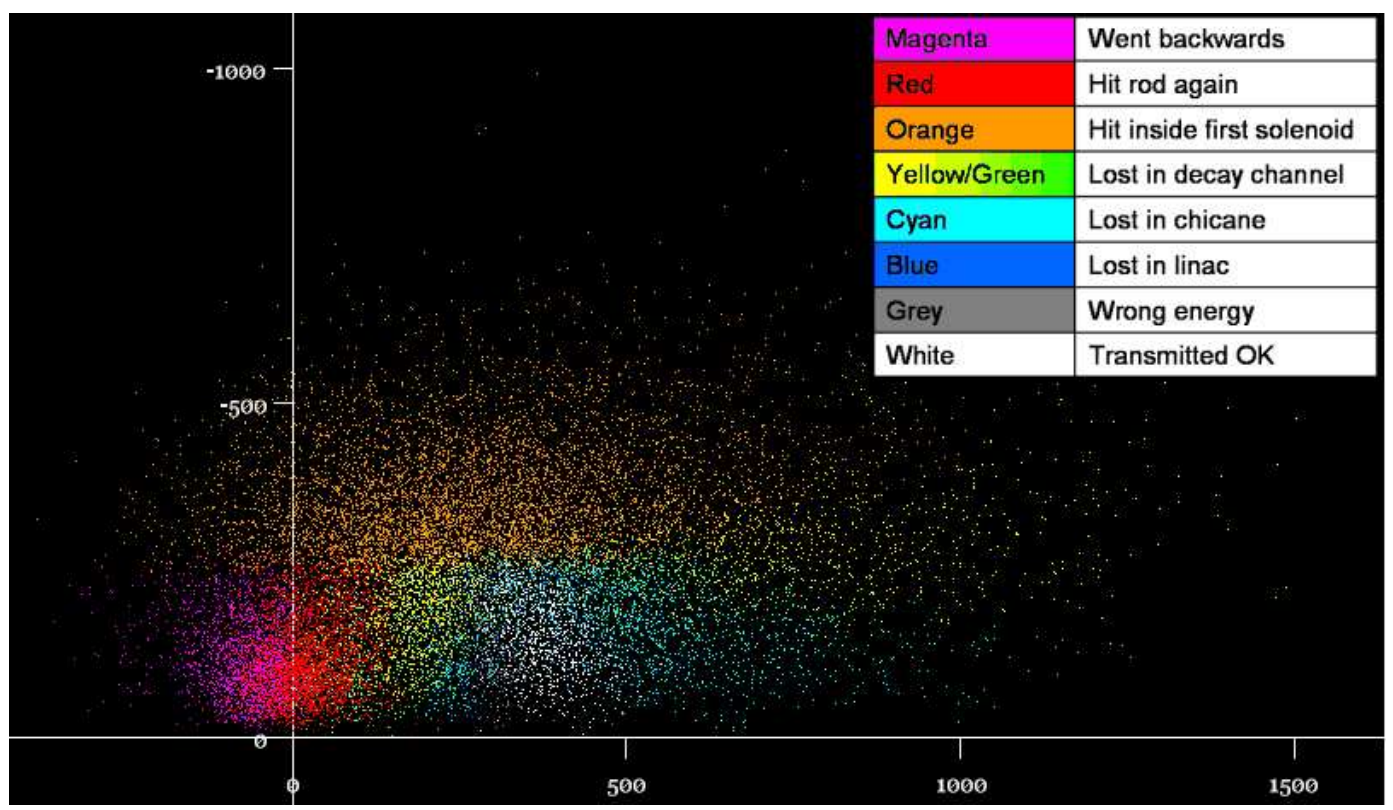

Figure 3.11: $\pi^{+} / \pi^{-}$fate along $2.2 \mathrm{GeV}$ optimised Chicane/Linac Channel, $P_{\text {long }}$ vs $P_{\text {transv }}$ [58]

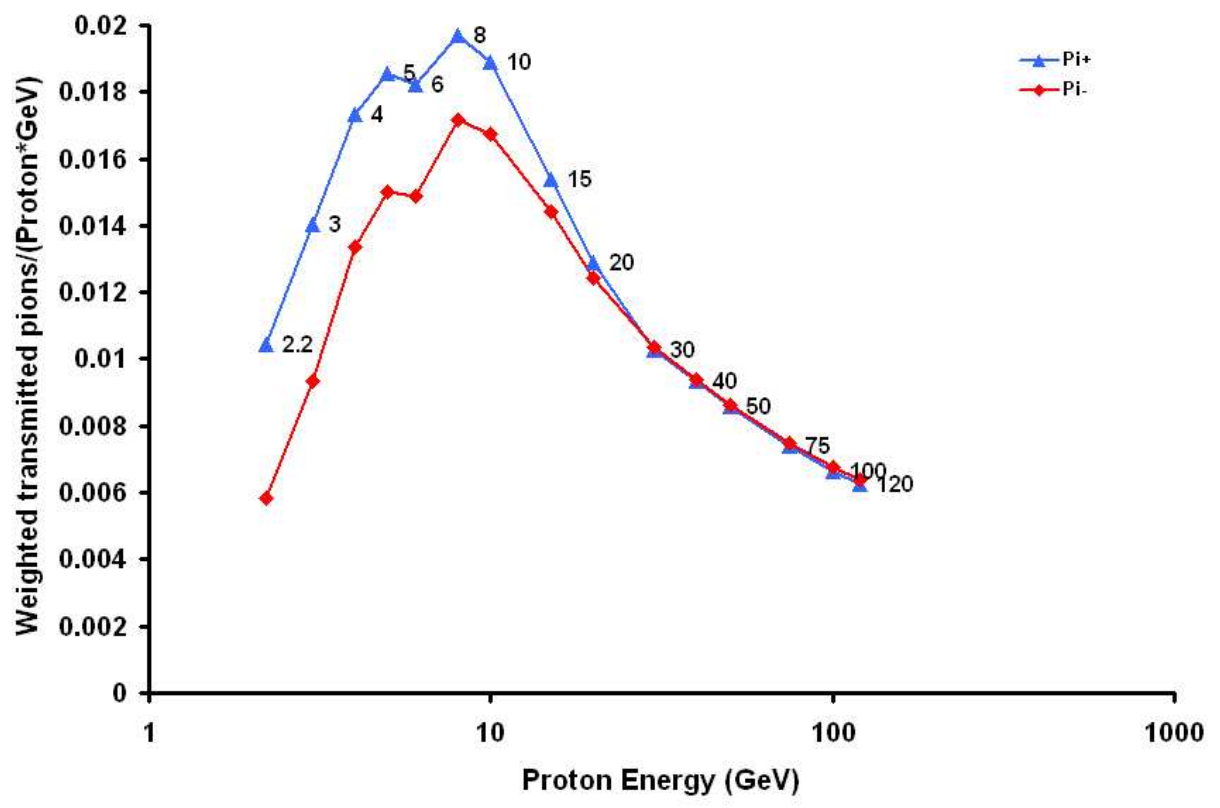

Figure 3.12: $\pi^{+} / \pi^{-}$transmission along $2.2 \mathrm{GeV}$ optimised Chicane/Linac Channel using a GEANT4 QGSP model.

(Figure 3.12) show that more $\pi^{+}$than $\pi^{-}$per proton beam power are transported until $30 \mathrm{GeV}$ where they are transported in equal measures. The peak appears to be around $8 \mathrm{GeV}$ although the dip which appears for both $\pi^{ \pm}$at $6 \mathrm{GeV}$ seems strange and probably non-physical. Negative pions are preferentially transported along the channel at the very highest energies. 


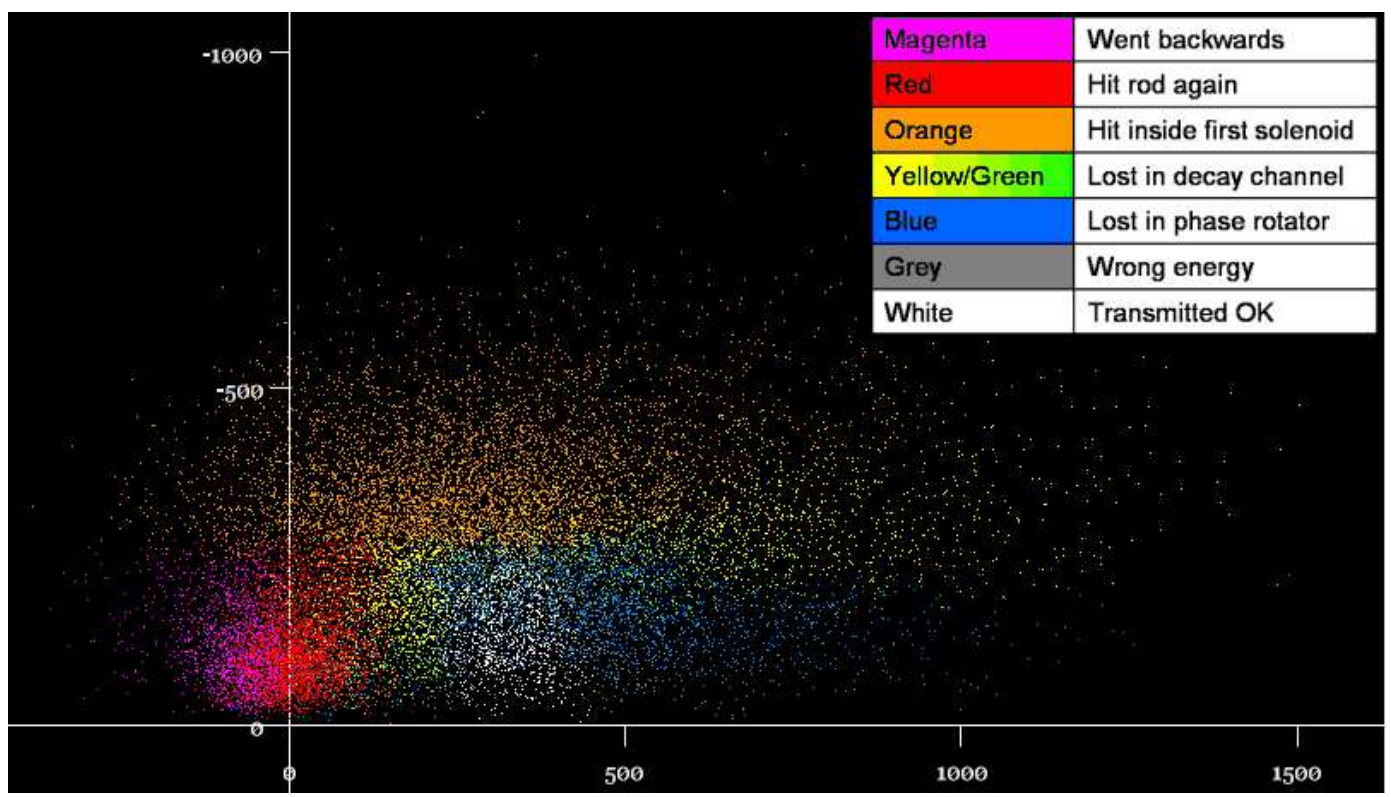

Figure 3.13: $\pi^{+} / \pi^{-}$fate along $2.2 \mathrm{GeV}$ optimised Phase Rotator Channel, $P_{\text {long }}$ vs $P_{\text {transv }}$ [58]

The results for the Phase Rotator channel are shown in Figure 3.14. It can be seen that many of the features of the Chicane/Linac plot are evident here also. Namely, the peak at 8 $\mathrm{GeV}$, the dip at $6 \mathrm{GeV}$, the excess of $\pi^{+}$transmitted compared to $\pi^{-}$until $30 \mathrm{GeV}$ and the behavior at higher energies. It can be seen that the number of both pion charge conjugates transmitted differ by only a few percent between schemes at $2.2 \mathrm{GeV}$. This is not surprising as it is at the momentum for which both schemes were optimised. On the other hand at the $8 \mathrm{GeV}$ peak the Phase Rotation scheme only transmits around $90 \%$ of the pion transmission achieved by the Chicane Linac scheme.

\subsection{Hadronic model comparison of transmission}

The program ProbabilityMap was also run on the data-sets produced using the different GEANT4 use-cases detailed in section 3.4. Both the Chicane/Linac and Phase Rotator maps were used to calculate the transmission for the respective channels.

The transmission results for the Chicane/Linac scheme can be seen in Figure 3.15. It is noticeable that the binary cascade models have a higher transmission of pions although this is to be expected as these models produced the largest raw pion yields (see section 3.4). They also predict a higher transmission at lower energies which is also the energy domain with the 


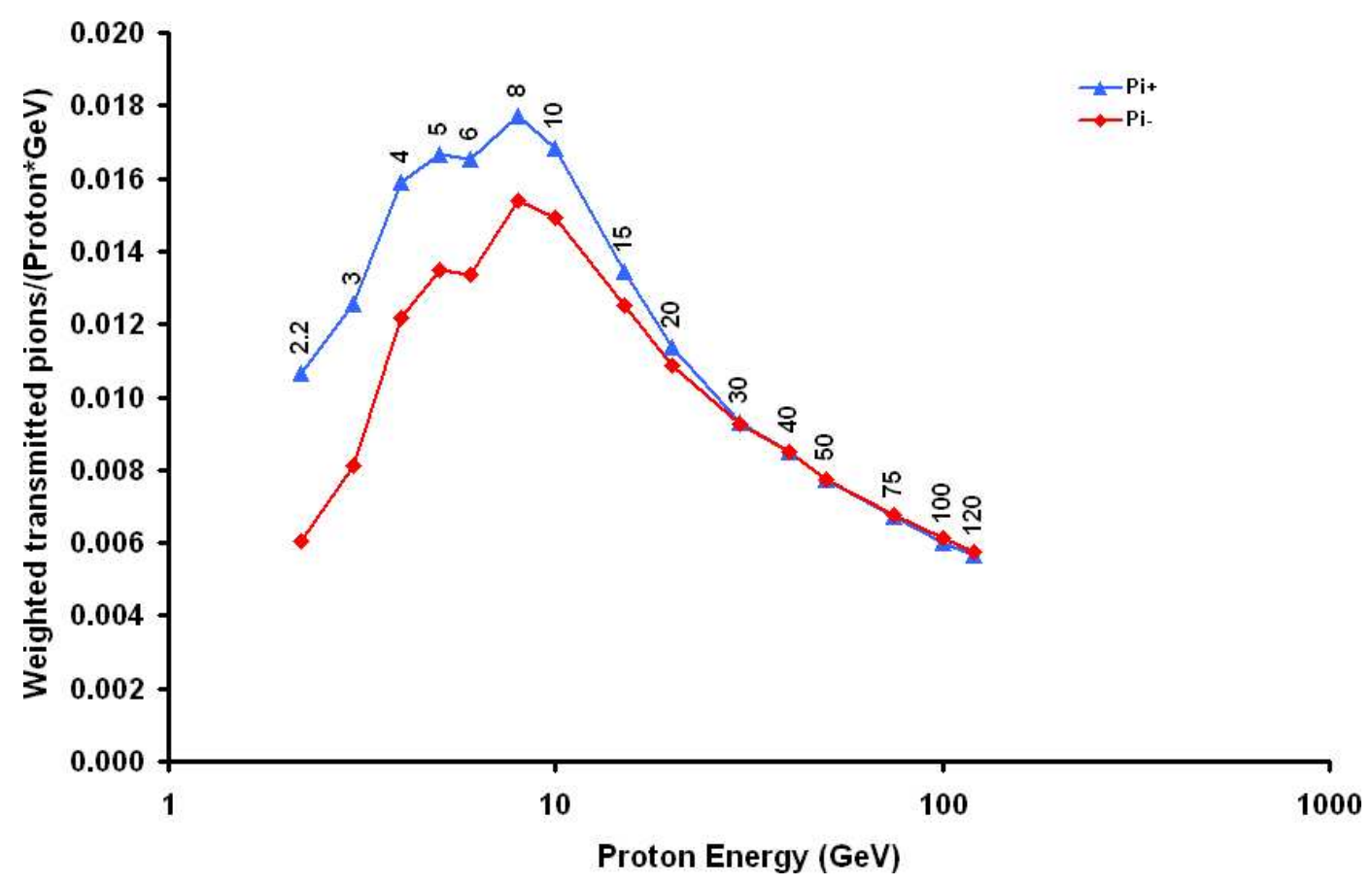

Figure 3.14: $\pi^{+} / \pi^{-}$transmission along $2.2 \mathrm{GeV}$ optimised Phase Rotation Channel using a GEANT4 QGSP model

largest raw yield. The LHEP and QGSP models also perform as the raw pions yields in section 3.4 would suggest with a peak around $8 \mathrm{GeV}$. It is interesting to note that the transmission of the data sets produced by the Bertini cascade models has little or no energy dependence in the range $2.2 \mathrm{GeV} \rightarrow 8 \mathrm{GeV}$. The sharp rise between $8 \mathrm{GeV}$ and $10 \mathrm{GeV}$ is likely due to a model transition region.

The transmission results for the phase rotator channel can be seen in Figure 3.16. The trends in the data in this plot are similar to the chicane/linac channel. One key difference is that across almost the entire energy scale studied the phase rotator transmission is lower that the chicane/linac equivalent for all non-Bertini cascade models. Figure 3.17 shows the percentage change in transmission between the both optical channels (per proton* $\mathrm{GeV}$ ) normalised to the chicane/linac channel for the hadronic models discussed. Positive values are in favour of the non-cooling chicane/linac channel, negative values in favour of the phase-rotator scheme. It can be seen that at $2.2 \mathrm{GeV}$ the QGSP and LHEP $\pi^{ \pm}$transmission for the phase rotation channnel is between $2-3 \%$ greater than that of the chicane/linac channel. For energies greater than this the difference is between $8-13 \%$ in favour of the chicane/linac channel. The transmission of pions created by binary cascade models is consistently greater for the chicane/linac scheme, 
differences being in the range $8 \% \rightarrow 10 \%$. The transmission of pions created by the Bertini cascade models for both schemes are shown to agree to roughly $5 \%$ across the entire energy range studied. The phase rotator channel performs better across the majority of proton energies with the only exception being at $10 \mathrm{GeV}$ where the difference is approximately $10 \%$ in favour of the chicane/linac scheme.

These results suggest that the chicane/linac channel is better at transmitting off momentum $\pi^{+}$and $\pi^{-}$than the phase rotator channel. This is the case for all non-Bertini Cascade hadron production models. These results are of course preliminary. A more rigorous comparison between transmission at different proton driver energies and between both channels at those energies can only be conducted when those channels are optimised to the relevant momentum magnetic lattices.

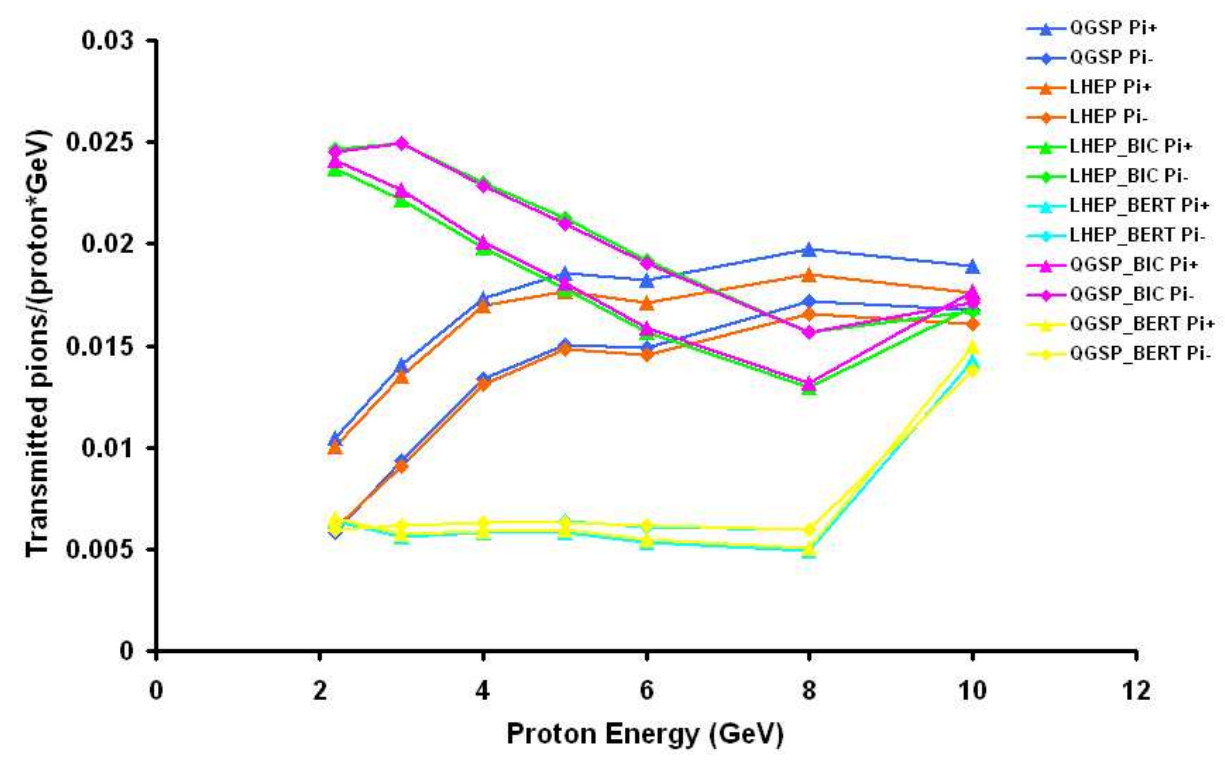

Figure 3.15: Comparison between GEANT4 hadronic model use-cases for $\pi^{+} / \pi^{-}$transmission along 2.2GeV optimised Chicane/Linac Channel

\subsection{Conclusion}

The results from this study suggest that the optimal proton energy for a Neutrino Factory derived using GEANT4 and adopting the QGSP hadronic algorithms is $10-15 \mathrm{GeV}$. It can be 


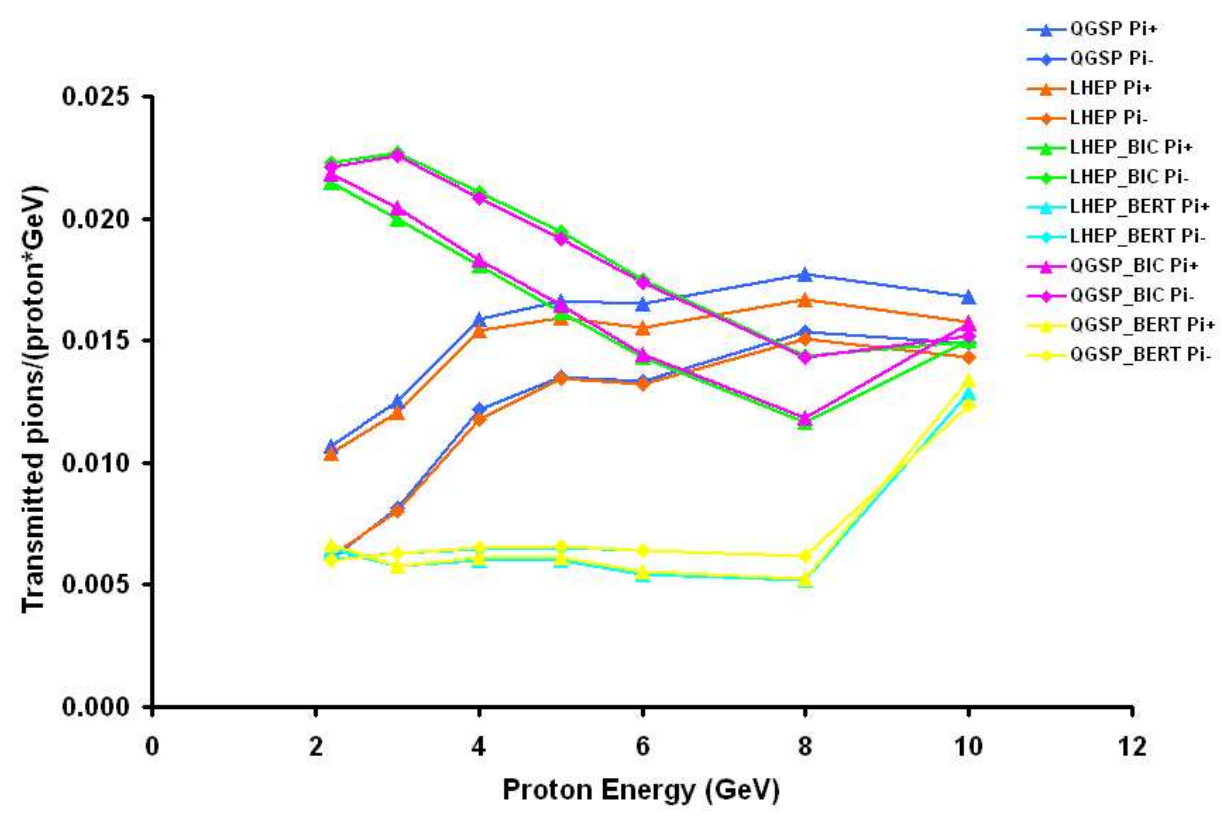

Figure 3.16: Comparison between GEANT4 hadronic model use-cases for $\pi^{+} / \pi^{-}$transmission along $2.2 \mathrm{GeV}$ optimised Phase Rotation Channel

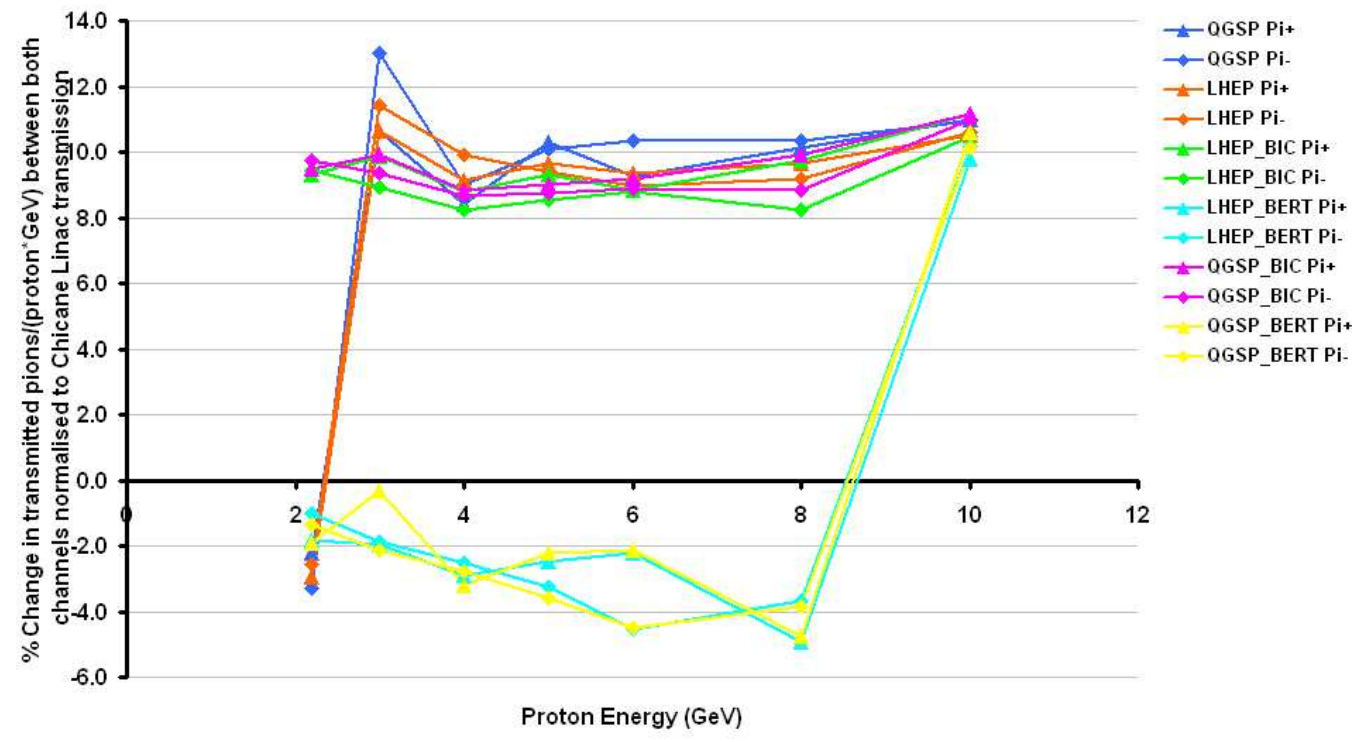

Figure 3.17: Percentage difference in "beam-power-normalised" transmission between Chicane/Linac and Phase Rotator channels. Percentage normalised to Chicane/Linac transmission

seen that within the GEANT4 physics use cases there is a large amount of variation in pion yields at low energies from different hadronic models. In terms of comparison between GEANT4 
and MARS codes, there are differences in pion yields at all energies with the differences being greatest at the lower end of the range studied. MARS data, as shown in [56] would suggest an optimal energy of around $30 \mathrm{GeV}$ for raw yields without probability grids. The largest difference between the two codes is in the calculated angular distributions for outgoing pions. GEANT4 appears to forward focus these pions to a much greater extent than MARS, which in turn leads to much greater yields of capturable pions ${ }^{2}$. To conclude, these results suggest the optimal proton driver energy for a neutrino factory is in the range 10-15 GeV, while MARS data suggests the optimal energy to be $30 \mathrm{GeV}$. Data from the HARP and other experiments is needed to resolve these discrepancies.

The results obtained by estimating the transmission through both the Chicane/Linac and Phase Rotator front ends show a peak in transmission around 8-10 GeV. It must be noted that the estimation assumes a $2.2 \mathrm{GeV}$ optimised channel. Both channels show major differences in transmission for different GEANT4 hadronic physics choices as would be expected from the raw yield studies. It appears that on the whole the Chicane/Linac channel is more efficient at transmitting off-momentum pions. Finally, it appears that for low proton driver energies in both front-ends, the transmission of Bertini Cascade model calculated pions is roughly independent of energy.

\footnotetext{
${ }^{2}$ Again with caveat on model validity
} 


\section{Chapter 4}

\section{Benchmarking of GEANT4 and MARS to HARP data}

\subsection{Purpose of HARP: Introduction}

A key consideration in the design of a Neutrino Factory proton driver is the hadron production yields. The lack of knowledge of proton-nucleon production at proton energies in the range 1.5 to $15 \mathrm{GeV}$ motivated the construction of the HAdRon Production experiment (HARP).

HARP is a fixed target experiment located at CERN, Geneva. In HARP (PS 216) protons are transported to the experiment from the CERN Proton Synchrotron (PS) via the T9 beamline [53]. The main physics goals of HARP are to investigate pion production cross-sections for incident proton energies of between 1.5-15 GeV for a number of different target nuclei, to determine the optimal proton energy at a neutrino factory, to determine particle yields for the $\mathrm{K} 2 \mathrm{~K}$ and MiniBoone experiments and to reduce the systematic error in the atmospheric neutrino fluxes.

\subsection{HARP Experimental Setup}

The HARP detector can be seen in Figure 4.1 and consists of the following elements [59]:

- Time projection chamber (TPC) within a solenoid to provide tracking and particle identification (PID) for highly transverse secondaries. The momentum measurement is derived 
from the measurement of the radius of curvature of the track and the PID is provided by energy loss in the gas $(\mathrm{dE} / \mathrm{dx})$.

- Resistive plate chambers for PID of 150 - $250 \mathrm{MeV} / \mathrm{c}$ electrons and pions [60]. At this momentum the $\mathrm{dE} / \mathrm{dx}$ for electrons and pions in the TPC is the same and does not allow particle tagging.

- Spectrometer constructed around a dipole magnet of $\int B_{y} d L=0.66 \mathrm{Tm}$

- Large drift chambers from the NOMAD experiment (NDC) for low angle secondaries [61].

- A time-of-flight wall (TOFW), a threshold Cherenkov detector (CHE) and an electromagnetic calorimeter (ECAL) which are all used for particle identification. TOFW measures target-wall flight-time, CHE discriminates between high momentum pions and protons and the ECAL discriminates between electrons and pions above the Cherenkov threshold. The ECAL can also identify beam muons which could be mis-identified as pions and as such detrimentally affect the precision of the calculated pion cross-section. The beam muons at high momentum are identified by demanding no showering in the muon identifier and at low momentum (generally lower due to being produced from pion decay) by the fact that they are bent more by the dipole field.

- Different targets (located inside the TPC), that could be changed for different runs.

- Three beam timing detectors (BTOF). The BTOF detectors were time of flight hodoscopes along the beamline that allowed separation of pions, kaons and protons up to $5 \mathrm{GeV} / \mathrm{c}$

- Two threshold Cherenkov detectors (BCA and BCB) which were used to identify electrons and pions at high energy

A detailed description of the HARP detector can be found in [?] and a description of the particle identification algorithms can be found in [62].

\subsection{Simulation}

The first results from HARP involved the illumination of an aluminium target by a proton beam of $12.9 \mathrm{GeV} / \mathrm{c}$ [63]. This data can be used to validate both GEANT4 and MARS particle tracking 


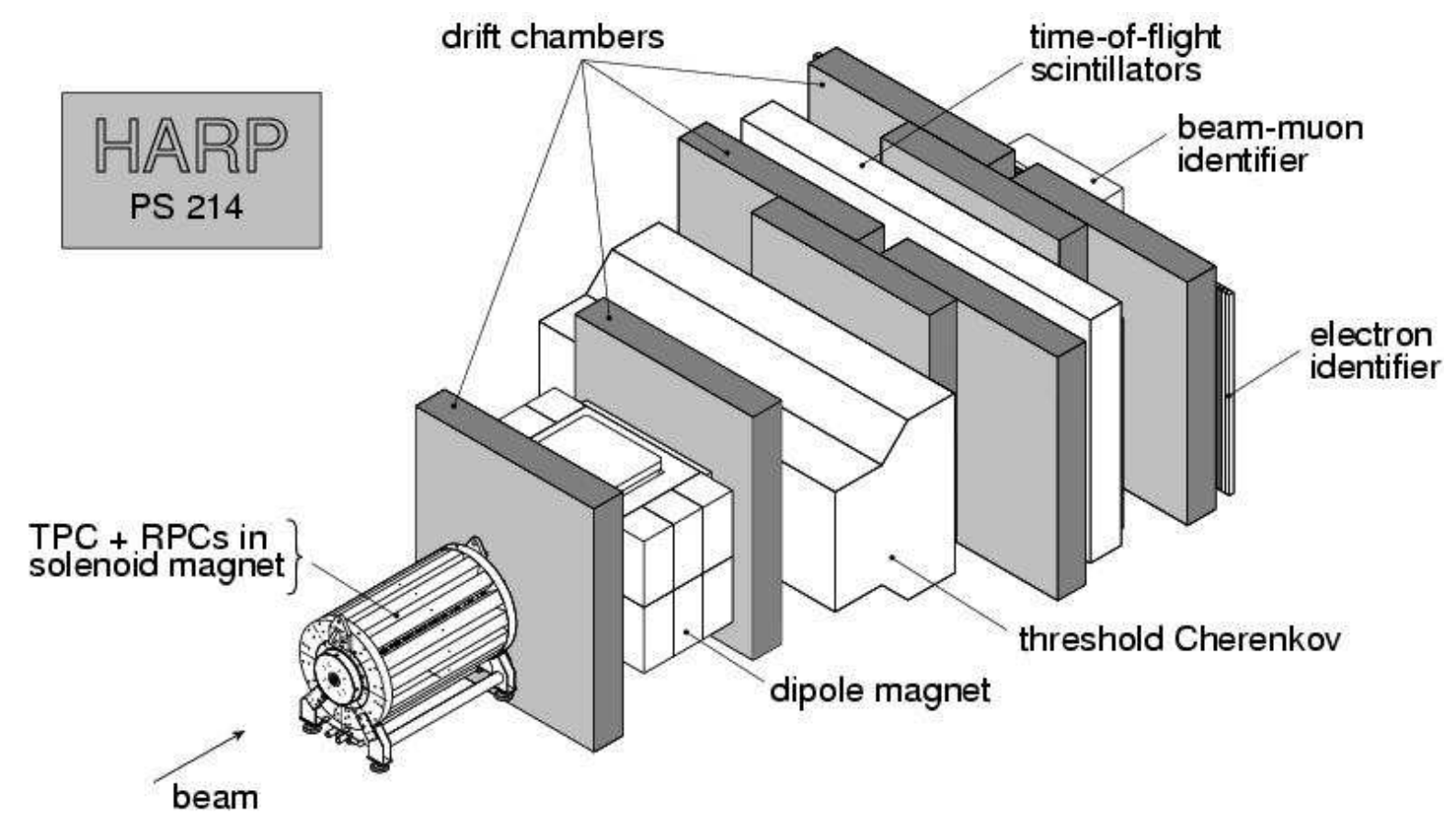

Figure 4.1: The layout of the HARP experiment

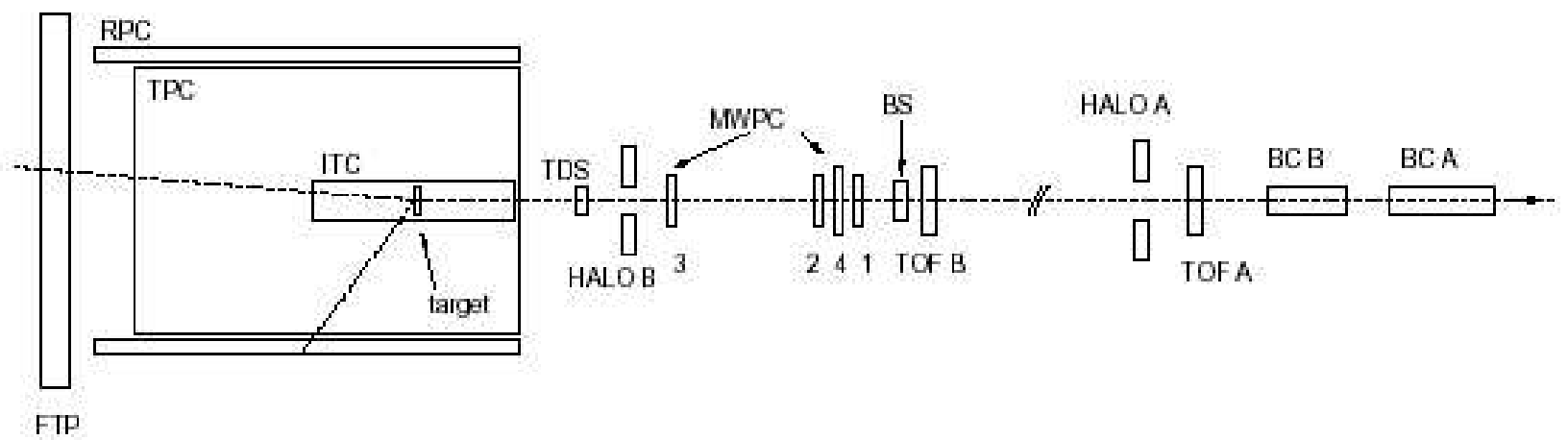

Figure 4.2: The HARP beam-trigger layout

codes in this regime and in the case of GEANT4 discriminate between hadronic engines at this energy. The results presented in [63] are of the double-differential cross-section $\left(d^{2} \sigma^{\pi^{+}} / d p d \Omega\right)$ of $\pi^{+}$production by interaction of protons incident on an aluminium target of $5 \%$ nuclear interaction length. The results reported are for pion secondaries in the momentum range 0.75 - $6.5 \mathrm{GeV} / \mathrm{c}$ and within the angular range 30 - $210 \mathrm{mrad}$. This study will act to benchmark the target results shown previously. Preferably the comparison would use data produced for proton interactions with a tantalum target but currently the only data available by HARP is 
the proton-aluminium data ${ }^{1}$.

The trigger and event selection criteria used by HARP are detailed in [63]. A summary of the reconstructed events can be seen in Table 4.1.

\begin{tabular}{|l|l|l|}
\hline Requirement of data & $\mathrm{Al} 5 \% \lambda_{I}$ at $12.9 \mathrm{GeV} / \mathrm{c}$ & $12.9 \mathrm{GeV} / \mathrm{c}$ empty target \\
\hline \hline Protons on target & 17954688 & 4769408 \\
Total events processed & 4710609 & 771330 \\
Events with accepted beam pro- & 3404372 & 547838 \\
ton & & \\
Prescaled triggers with accepted & 280542 & 74522 \\
beam proton & & \\
FTP triggers & 2087732 & 225639 \\
FTP trigger rate (triggers/p.o.t) & 0.116 & 0.047 \\
Total good tracks & 209929 & 11704 \\
\hline
\end{tabular}

Table 4.1: Number of events for different HARP data sets with $5 \% \lambda_{I}$ aluminium target and without. For details of trigger selection criteria see [63]

\subsection{Simulations using GEANT4}

To compare the performance of GEANT4 with the available HARP data a simulation of the HARP target was carried out using the tools that produced the results in [58]. The simulation was implemented in G4Beamline. A program which converted G4Beamline output to a Root file was then created and the analysis carried out using the CERN Root package [66]. In the simulation a solid cylindrical aluminium target volume was constructed. The dimensions of the target were $15 \mathrm{~mm}$ in radius and $18.76 \mathrm{~mm}$ in length. This geometry was chosen to directly mimic the HARP target. The target length was chosen as it is the midpoint between the maximum variation in the thickness as measured for the HARP target, the range being 19.73 to $19.85 \mathrm{~mm}$ [63]. The target was then illuminated by a Gaussian beam of 4 million protons with momentum distribution of $12.9 \mathrm{GeV} \pm 129 \mathrm{MeV} / \mathrm{c}$. Again this is to try to reproduce the proton beam incident on HARP in which the momentum is known to $1 \%$ [63].

\footnotetext{
${ }^{1}$ As this thesis was being published, HARP data of positive pion from $8.9 \mathrm{GeV} / \mathrm{c}$ protons on beryllium [64] and charged pions by protons on a tantalum target [65] have been submitted for publication
} 


\begin{tabular}{|l|l|}
\hline \multicolumn{2}{|c|}{ Physical properties of G4beamline simulation } \\
\hline \hline $\begin{array}{l}\text { Gaussian proton } \\
\text { beam }\end{array}$ & $\langle P\rangle=12.9 \mathrm{GeV}, \sigma_{P}=129 \mathrm{MeV}, \sigma_{x}=\sigma_{y}=\sigma_{x^{\prime}}=\sigma_{y^{\prime}}=0$ \\
$\begin{array}{l}\text { Target material } \\
\text { Target dimensions }\end{array}$ & $\begin{array}{l}\text { Aluminium, } \mathrm{z}=13, \mathrm{~A}=26.9815, \text { density }=2.699 \mathrm{~g} / \mathrm{cm}^{3} \\
\text { Radius }=15 \mathrm{~mm}, \text { length }=19.76 \\
\text { tor plane }\end{array}$ \\
\hline
\end{tabular}

Table 4.2: Geant4 simulation parameters

\subsection{Calculation of differential cross-section}

In the HARP analysis the expression for the double-differential cross-section for the production of a particle of type $\alpha$ is as follows:

$$
\frac{d^{2} \sigma_{\alpha}}{d p_{i} d \theta_{j}}=\frac{1}{N_{p o t}} \frac{A}{N_{A} \rho t} M_{i j \alpha i^{\prime} j^{\prime} \alpha^{\prime}}^{-1} \cdot N_{i^{\prime} j^{\prime}}^{\alpha^{\prime}}
$$

where $N^{\alpha^{\prime}{ }^{\prime} j^{\prime}}$ is the number of particles tagged of type $\alpha^{\prime}$ in reconstructed 2D momentum $\left(p_{i^{\prime}}\right) \times \operatorname{angular}\left(\theta_{j^{\prime}}\right)$ bins. $M_{i j \alpha i^{\prime} j^{\prime} \alpha^{\prime}}^{-1}$ is a correction matrix which corrects for detector efficiency and resolution. This construction takes care of unfolding the true variables ij $\alpha$ from the reconstructed variables $\left(i^{\prime} j^{\prime} \alpha\right)$. This is motivated by corrections due to acceptance, absorption, pion decay, reconstruction, tertiary production, particle identification efficiency and particle misidentification rate. $\frac{A}{N_{A} \rho t}$ is the inverse of the target nuclei per unit area (A is atomic mass, $N_{A}$ is the Avogadro number, $\rho$ the target density and the thickness). $N_{\text {pot }}$ is the number of protons on target which for the aluminium run was 17.95 Million.

For the GEANT4 and MARS simulations carried out in this paper as we only deal with Monte Carlo truth this expression simplifies to:

$$
\frac{d^{2} \sigma_{\alpha}}{d p_{i} d \theta_{j}}=\frac{1}{N_{p o t}} \frac{A}{N_{A} \rho t} \cdot N_{i j}^{\alpha}
$$

where $N_{i j}^{\alpha}$ is the raw yield of particle type $\alpha$ in $2 \mathrm{D}$ bin $p_{i} \times \theta_{j}$ and all other variables are as above.

Initially both the GEANT4 and MARS simulations were carried out with a $5 \%$ interaction length. This was to mimic directly the conditions of the HARP experiment. It was then decided that in order to eliminate the effect of secondary reabsorption in the target we should exprapolate 
this result to zero interaction length. To do this an identical simulation was carried out with a target of $1 \%$ interaction length. A linear extrapolation to zero interaction length was then carried out. All results shown in this document have been extrpolated to zero interaction length. In the HARP analysis reabsorption effects were folded into the correction matrix. 


\subsection{Harp positive pion double-differential cross- section results summary}

The HARP analysis was carried out on 210000 secondary tracks that were reconstructed in the forward spectrometer. The results of this analysis can be seen in Table 4.3.

\begin{tabular}{|c|c|c|c|c|}
\hline$\theta_{\min }(\operatorname{mrad})$ & $\theta_{\max }(\operatorname{mrad})$ & $p_{\min }(G e V / c)$ & $p_{\max }(G e V / c)$ & $d^{2} \sigma^{\pi_{H A R P}^{+}} / d p d \Omega(\mathrm{mb} /(\mathrm{GeV} / \mathrm{c} \mathrm{sr}))$ \\
\hline \multirow[t]{8}{*}{30} & \multirow[t]{8}{*}{60} & 0.75 & 1.25 & $410 \pm 56$ \\
\hline & & 1.25 & 1.75 & $473 \pm 49$ \\
\hline & & 1.75 & 2.25 & $465 \pm 41$ \\
\hline & & 2.25 & 2.75 & $441 \pm 33$ \\
\hline & & 2.75 & 3.25 & $464 \pm 29$ \\
\hline & & 3.25 & 4.00 & $346 \pm 18$ \\
\hline & & 4.00 & 5.00 & $284 \pm 18$ \\
\hline & & 5.00 & 6.50 & $129.7 \pm 8.1$ \\
\hline \multirow[t]{8}{*}{60} & \multirow[t]{8}{*}{90} & 0.75 & 1.25 & $412 \pm 42$ \\
\hline & & 1.25 & 1.75 & $456 \pm 42$ \\
\hline & & 1.75 & 2.25 & $456 \pm 36$ \\
\hline & & 2.25 & 2.75 & $407 \pm 24$ \\
\hline & & 2.75 & 3.25 & $381 \pm 19$ \\
\hline & & 3.25 & 4.00 & $249 \pm 13$ \\
\hline & & 4.00 & 5.00 & $176 \pm 13$ \\
\hline & & 5.00 & 6.50 & $68.9 \pm 6.3$ \\
\hline \multirow[t]{8}{*}{90} & \multirow[t]{8}{*}{120} & 0.75 & 1.25 & $429 \pm 45$ \\
\hline & & 1.25 & 1.75 & $442 \pm 36$ \\
\hline & & 1.75 & 2.25 & $384 \pm 26$ \\
\hline & & 2.25 & 2.75 & $330 \pm 20$ \\
\hline & & 2.75 & 3.25 & $287 \pm 15$ \\
\hline & & 3.25 & 4.00 & $164.7 \pm 9.8$ \\
\hline & & 4.00 & 5.00 & $105.4 \pm 8.1$ \\
\hline & & 5.00 & 6.50 & $41.4 \pm 4.3$ \\
\hline
\end{tabular}




\begin{tabular}{|c|c|c|c|c|}
\hline \multirow[t]{8}{*}{120} & 150 & 0.75 & 1.25 & $434 \pm 44$ \\
\hline & & 1.25 & 1.75 & $404 \pm 31$ \\
\hline & & 1.75 & 2.25 & $329 \pm 23$ \\
\hline & & 2.25 & 2.75 & $258 \pm 18$ \\
\hline & & 2.75 & 3.25 & $213 \pm 13$ \\
\hline & & 3.25 & 4.00 & $119.1 \pm 7.9$ \\
\hline & & 4.00 & 5.00 & $62.8 \pm 5.2$ \\
\hline & & 5.00 & 6.50 & $24.2 \pm 3.4$ \\
\hline \multirow[t]{8}{*}{150} & 180 & 0.75 & 1.25 & $441 \pm 47$ \\
\hline & & 1.25 & 1.75 & $371 \pm 31$ \\
\hline & & 1.75 & 2.25 & $275 \pm 21$ \\
\hline & & 2.25 & 2.75 & $203 \pm 17$ \\
\hline & & 2.75 & 3.25 & $153 \pm 10$ \\
\hline & & 3.25 & 4.00 & $77.5 \pm 7.1$ \\
\hline & & 4.00 & 5.00 & $35.5 \pm 4.5$ \\
\hline & & 5.00 & 6.50 & $13.3 \pm 1.7$ \\
\hline \multirow[t]{8}{*}{180} & 210 & 0.75 & 1.25 & $332 \pm 35$ \\
\hline & & 1.25 & 1.75 & $270 \pm 26$ \\
\hline & & 1.75 & 2.25 & $189 \pm 19$ \\
\hline & & 2.25 & 2.75 & $130 \pm 14$ \\
\hline & & 2.75 & 3.25 & $87.8 \pm 7.1$ \\
\hline & & 3.25 & 4.00 & $38.3 \pm 3.4$ \\
\hline & & 4.00 & 5.00 & $16.6 \pm 1.7$ \\
\hline & & 5.00 & 6.50 & $10.4 \pm 3.2$ \\
\hline
\end{tabular}

Table 4.3: Results from the HARP experiment for the double-differential cross-section in the laboratory system $\left(d^{2} \sigma^{\pi_{H A R P}^{+}} / d p d \Omega\right)$. The values shown were calculated using the official HARP analysis discussed in [63] 


\subsection{GEANT4 double-differential cross-section $d^{2} \sigma^{\pi_{H A R P}^{+}} / d p d \Omega$ results}

The results of the GEANT4 simulation of the double-differential $\pi^{+}$cross-section can be seen in Figures 4.3,4.4,4.5,4.6,4.7,4.8. Also plotted are the data measured by HARP.

The first general comment that should be made upon inspection of these results are that the cross-sections calculated using both the quark-gluon-string model and the LHEP models both with binary cascade and Bertini cascade flavours generally agree to the level of a few percent. The quark-gluon-string model with chiral invariance does deviate from the other GEANT4 hadronic models and could possibly be argued to be the most accurate over our study range. This is certainly the case at low momentum and low angles. At the lowest angular bin in the study (3060mrad) QGSC performs significantly better that the other GEANT4 models. The disagreement between all GEANT4 models and the HARP data is more pronounced at the lower momenta with QGSC performing much better. This is also the general trend for secondaries in the 60-90 mrad range. For 60-120 mrad 4-6.5 GeV/c secondaries QGSC performs slightly poorer. In the 120-150 mrad angular range the measured double-differential cross-section for the lowest pion momenta created using each GEANT4 hadronic engines differs significantly from the HARP experimental data with QGSC out-performing the rest. All models perform approximately equivalently at high momenta. At relatively high angles 150-210 mrad we see further agreement between HARP and GEANT4 with QGSC dealing better with mid-momentum secondaries at the highest angles and though all hadronic models show significant underestimation of positive pion cross-sections at high angles. 


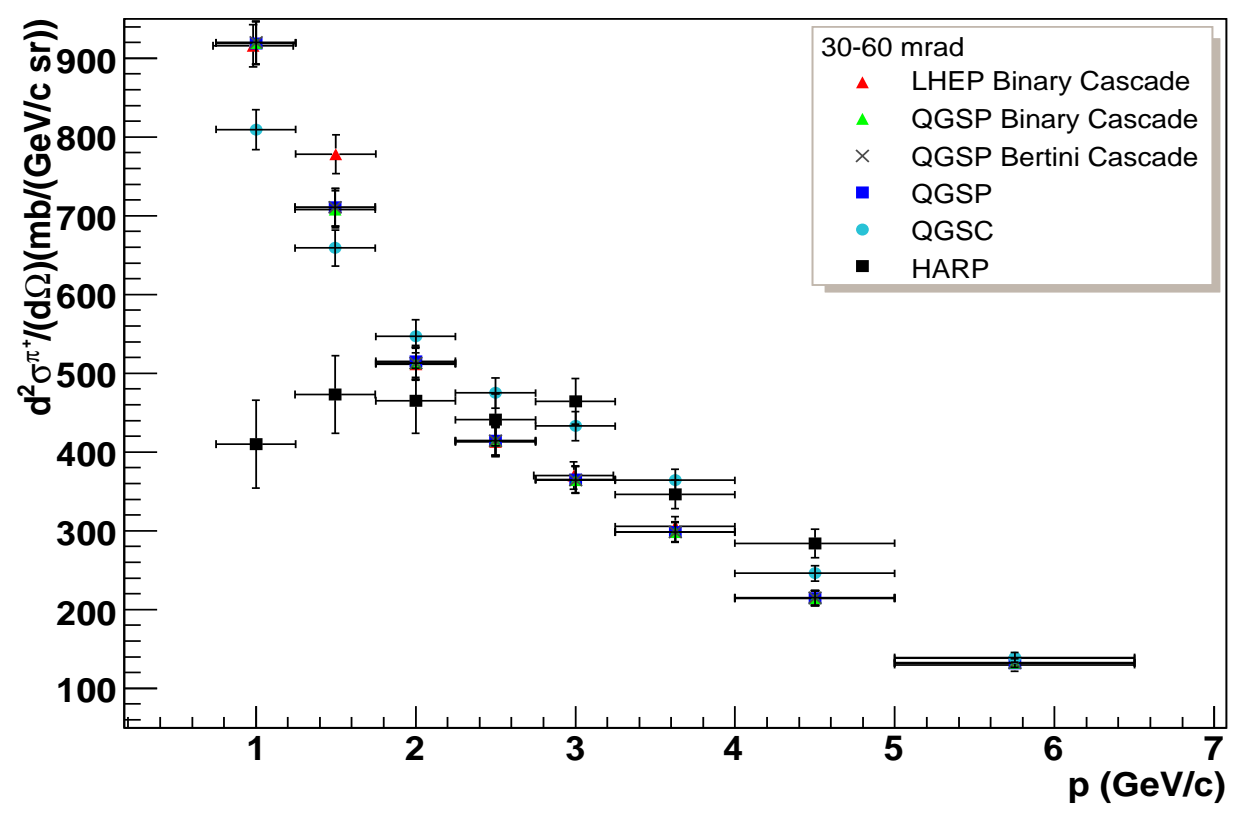

Figure 4.3: Measured double-differential $\pi^{+}$production cross-section $d^{2} \sigma /(d p d \Omega)$ for incoming protons of $12.9 \mathrm{GeV} / \mathrm{c}$ on an aluminium target as a function of pion momentum $\mathrm{p}$ for pion polar angle $30 \leq \theta<60 \mathrm{mrad}$. The results of GEANT4 simulations with different hadronic models are compared to the HARP experimental data

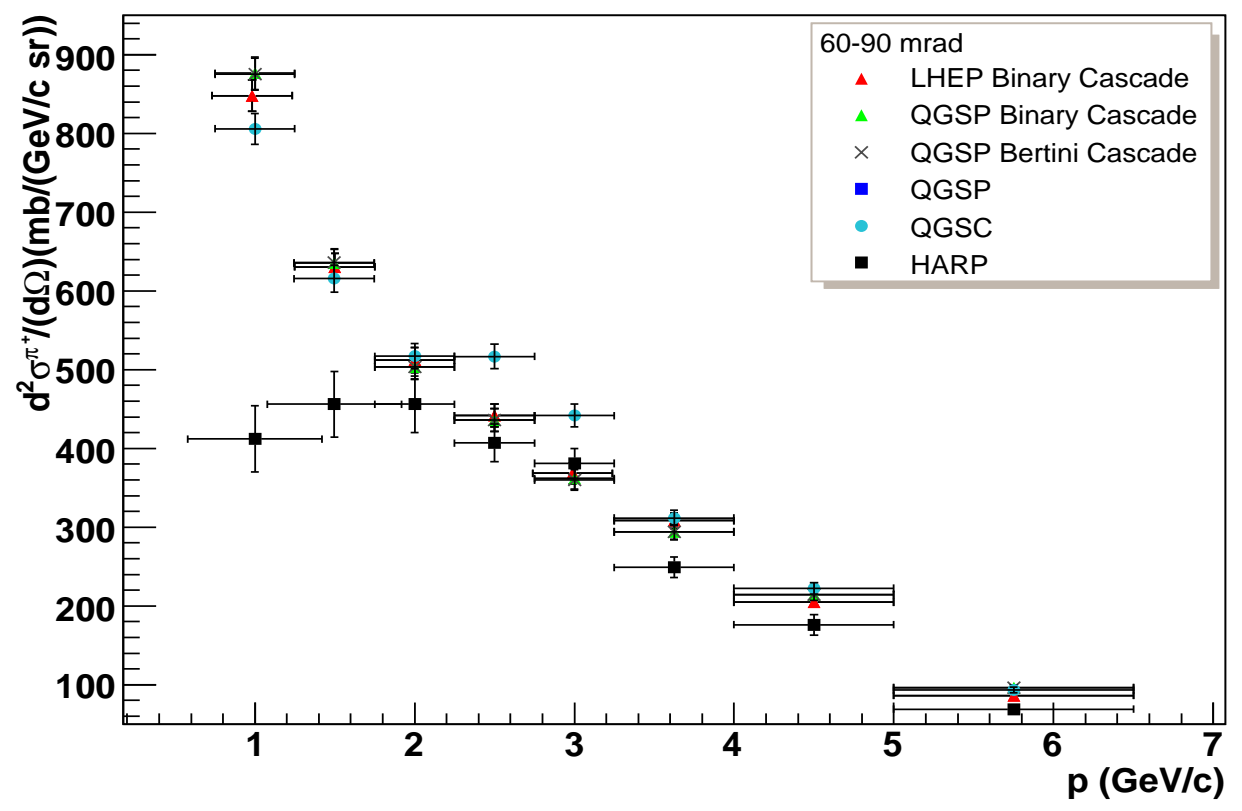

Figure 4.4: Measured double-differential $\pi^{+}$production cross-section $d^{2} \sigma /(d p d \Omega)$ for incoming protons of $12.9 \mathrm{GeV} / \mathrm{c}$ on an aluminium target as a function of pion momentum $\mathrm{p}$ for pion polar angle $60 \leq \theta<90 \mathrm{mrad}$. The results of GEANT4 simulations with different hadronic models are compared to the HARP experimental data 


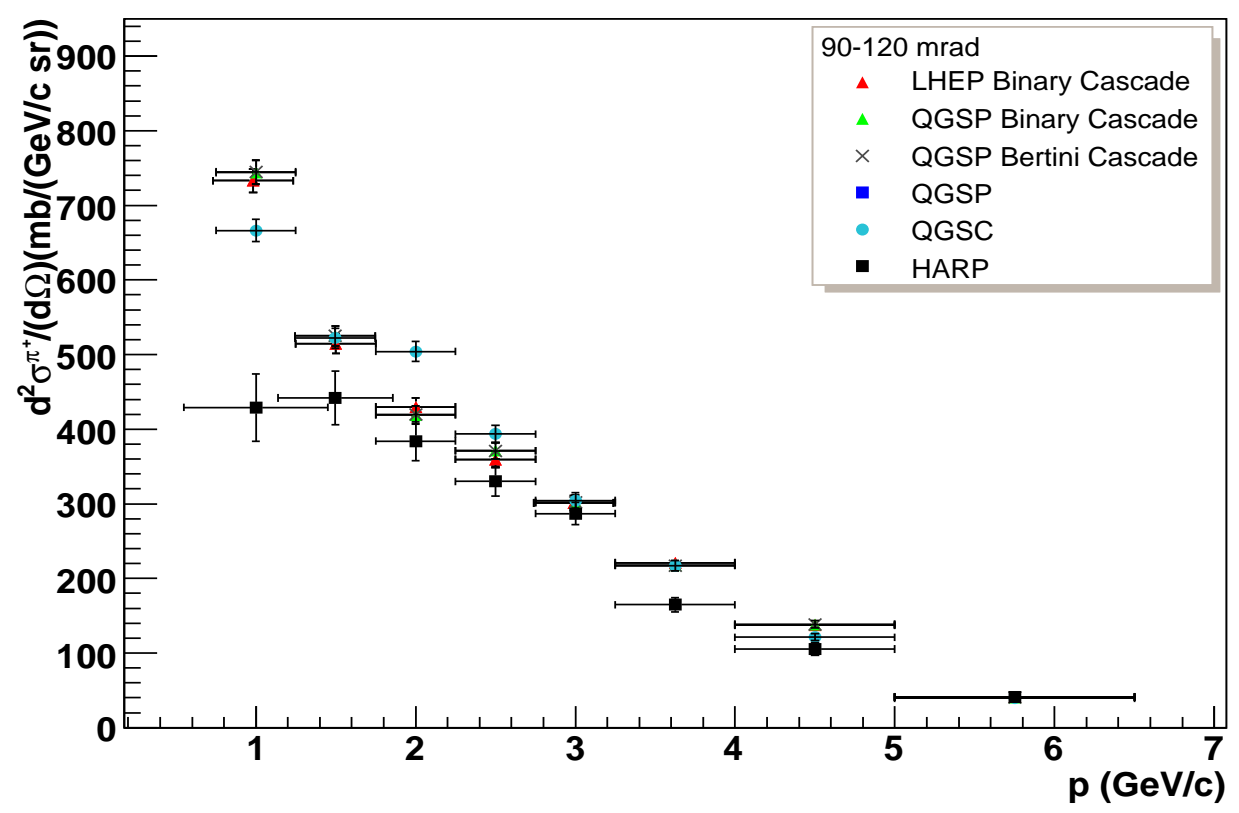

Figure 4.5: Measured double-differential $\pi^{+}$production cross-section $d^{2} \sigma /(d p d \Omega)$ for incoming protons of $12.9 \mathrm{GeV} / \mathrm{c}$ on an aluminium target as a function of pion momentum $\mathrm{p}$ for pion polar angle $90 \leq \theta<120 \mathrm{mrad}$. The results of GEANT4 simulations with different hadronic models are compared to the HARP experimental data

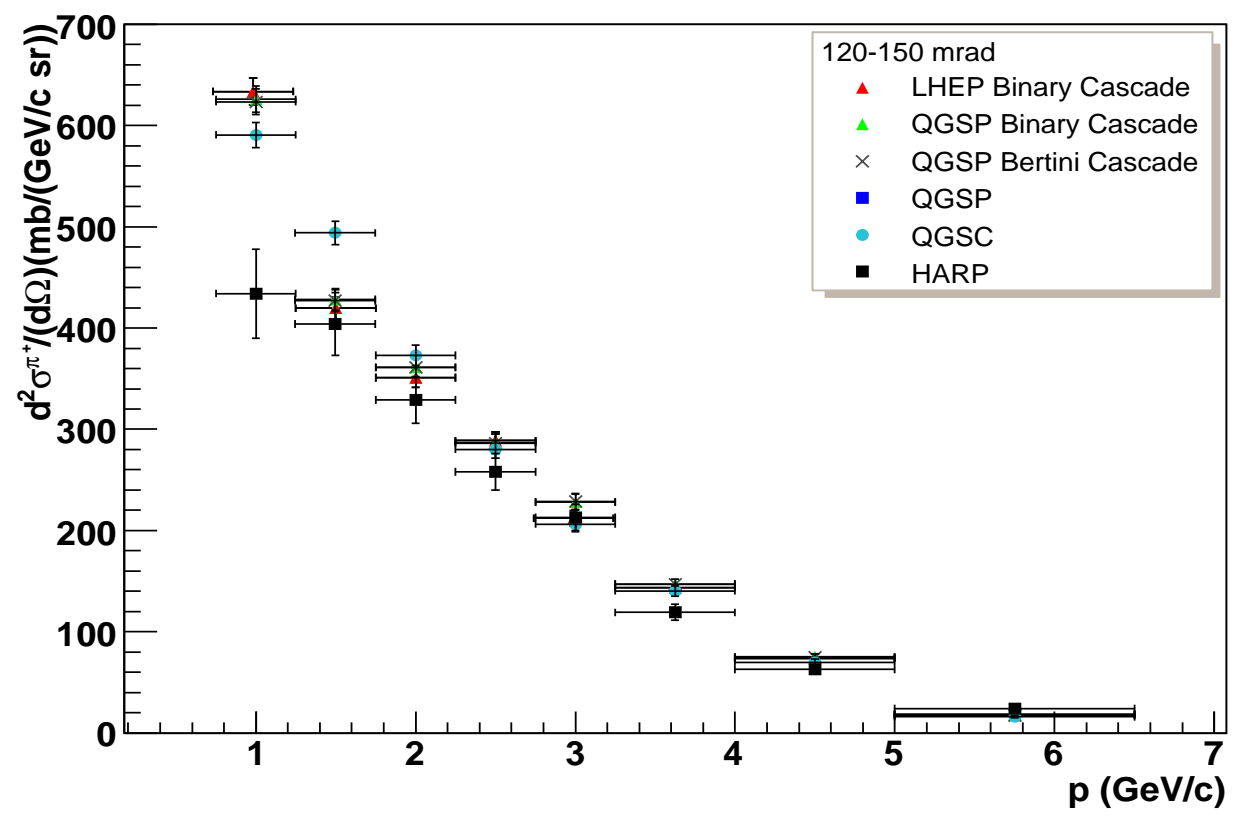

Figure 4.6: Measured double-differential $\pi^{+}$production cross-section $d^{2} \sigma /(d p d \Omega)$ for incoming protons of $12.9 \mathrm{GeV} / \mathrm{c}$ on an aluminium target as a function of pion momentum $\mathrm{p}$ for pion polar angle $120 \leq \theta<150 \mathrm{mrad}$. The results of GEANT4 simulations with different hadronic models are compared to the HARP experimental data 


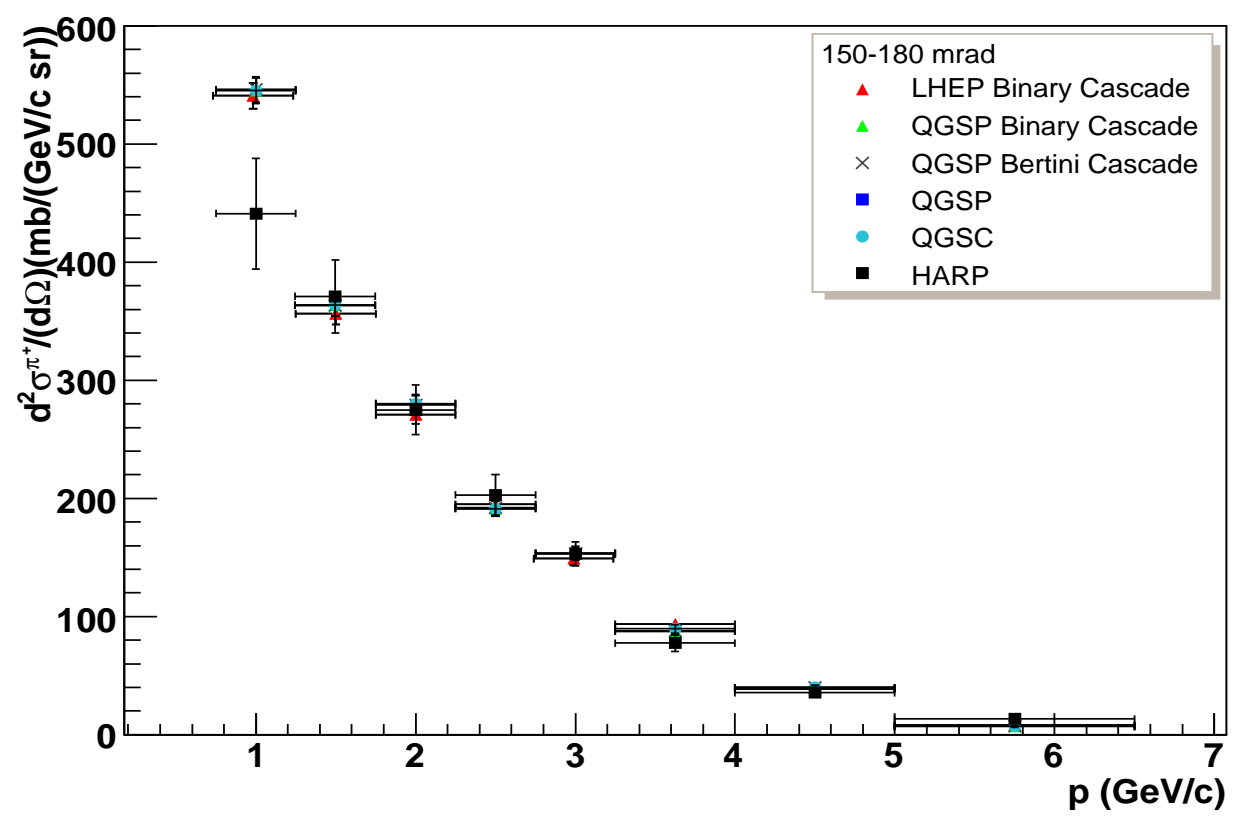

Figure 4.7: Measured double-differential $\pi^{+}$production cross-section $d^{2} \sigma /(d p d \Omega)$ for incoming protons of $12.9 \mathrm{GeV} / \mathrm{c}$ on an aluminium target as a function of pion momentum $\mathrm{p}$ for pion polar angle $150 \leq \theta<180 \mathrm{mrad}$. The results of GEANT4 simulations with different hadronic models are compared to the HARP experimental data

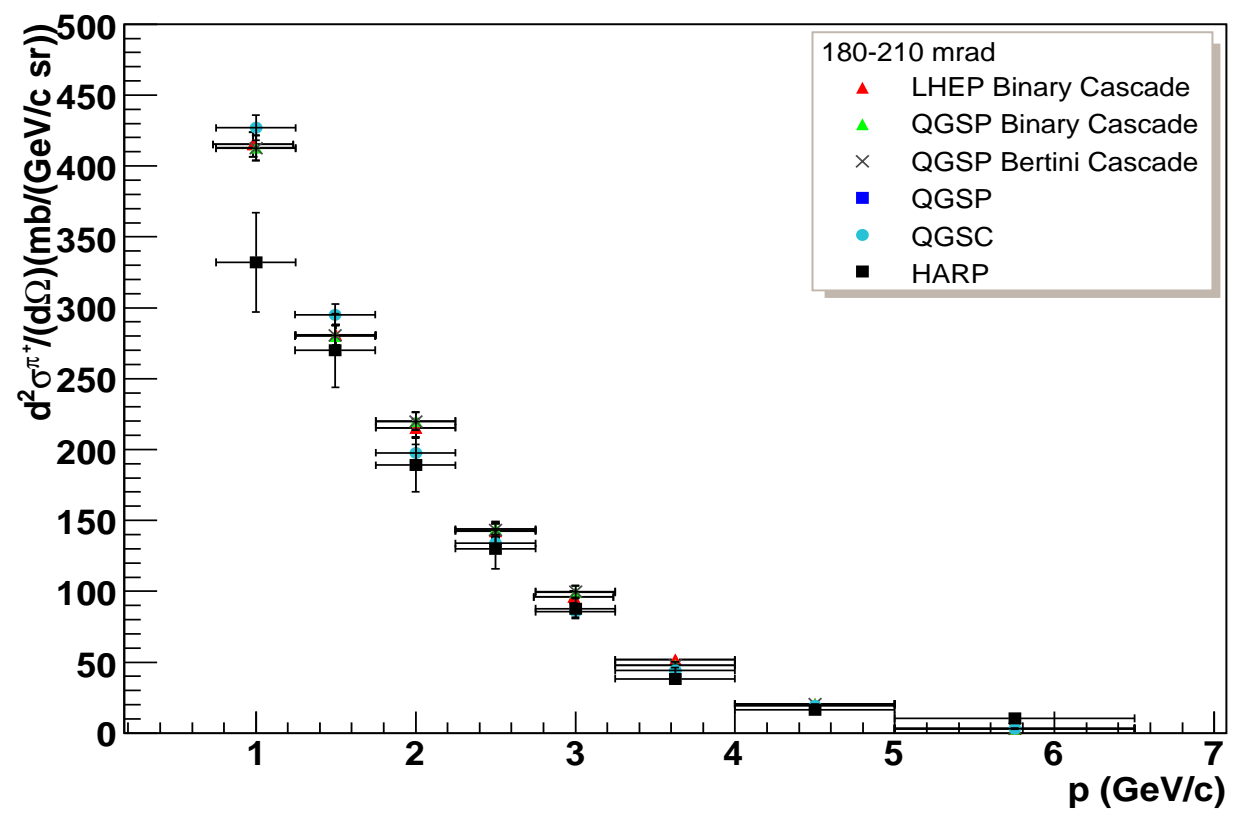

Figure 4.8: Measured double-differential $\pi^{+}$production cross-section $d^{2} \sigma /(d p d \Omega)$ for incoming protons of $12.9 \mathrm{GeV} / \mathrm{c}$ on an aluminium target as a function of pion momentum $\mathrm{p}$ for pion polar angle $180 \leq \theta<120 \mathrm{mrad}$. The results of GEANT4 simulations with different hadronic models are compared to the HARP experimental data 


\subsection{Results of comparison with MARS production}

\section{cross-sections}

The results of an identical Mars simulation can be seen in Figure 4.9. The simulation was carried out using the MARS15 code mentioned in the previous chapter. These results were calculated in the same manner as the GEANT4 results shown previously with simulations of a $1 \%$ and $5 \%$ interaction length target and then a linear extrapolation to zero interaction length. The extrapolated values of the double-differential $\pi^{+}$production cross-sections $d^{2} \sigma /(d p d \Omega)$ are those shown.

Inspection of the data shows that the general shape of the cross-section data produced using MARS are fairly consistent with the HARP data for all angles in our range (30-210 mrad) and for all momenta in our range $(0.75-6.5 \mathrm{GeV} / \mathrm{c})$. The trends of the production cross-sections seem to agree better than the GEANT4 equivalent trends. This is more pronounced when observing the flattening of the HARP data between $30-90$ mrad for $\leq 2.75 \mathrm{GeV} / \mathrm{c}$ secondaries. The MARS hadronic engine does appear to overestimate the $\pi^{+}$production in each bin analysed. Similarly to the GEANT4 data this increase is greater for the lowest momenta bins $(0.75-1.75 \mathrm{GeV} / \mathrm{c})$ and at angles closer to the beam axis. In this region the disagreement is at roughly the $50 \%$ level.

A direct comparison between the GEANT4-QGSC model and MARS15 can be seen in Figure 4.10. The percentage difference in each model normalised to the HARP data is shown. In the smallest angular bin (30-60 mrad) it is evident that both GEANT4 and MARS overestimate the cross-section to a large degree with QGSC and MARS at roughly the $+100 \%$ and $+50 \%$ level. For higher momenta pions the agreement is better at the $\pm 20 \%$ level with QGSC arguably performing better in this higher momentum regime. At 60-90 mrad the results show MARS to be performing better with agreement of a similar range to that in the lowest angular bin for $\pi^{+}>1.25 \mathrm{GeV} /$ c. QGSC performs well in this region with the percentage error at $+15-35 \%$. For the lowest momentum pions the disagreement is as poor as that found in the lowest bin. In the 90-120 mrad bin improvement is seen for the lowest momenta $\pi^{+}$with QGSC and MARS at the $+55 \%$ and $+7 \%$ respectively. MARS data in the mid-momentum region of the study is generally better that produced with the GEANT4 model. In the highest bin, GEANT4 data is 
substantially better. At 120-150 mrad there is further improvements in the QGSC production at low momentum with a further decrease to $+35 \%$. MARS is underestimating the pion production in this range at the $7 \%$ level. In the mid-momentum bins both QSGC/MARS agree with HARP in the range $\pm 20 \%$. The agreement between the HARP measured production of $5-6.5 \mathrm{GeV} / \mathrm{c}$ pions and that from both QGSC and MARS is becoming poor at 90-120 mrad with both codes underestimating production by $35 \%$. This underestimation is manifest in the 120-150 region also with the GEANT4 model performing worse at $-54 \%$. In the $120-150 \mathrm{mrad}, 0.75-1.25 \mathrm{GeV} / \mathrm{c}$ bin QGSC/HARP consistency improves further to show agreement at the $+22 \%$ level. For the midmomentum bins the QGSC/HARP agreement is good at $\pm 5 \%$. Finally for the largest angles (180-210 mrad) the results show QGSC to agree better than MARS in the mid-momentum range with both models showing poor agreement with HARP at the largest angles. Both models underestimating pion production with MARS and GEANT4-QGSC at the $50 \%$ and $70 \%$ level respectively. 

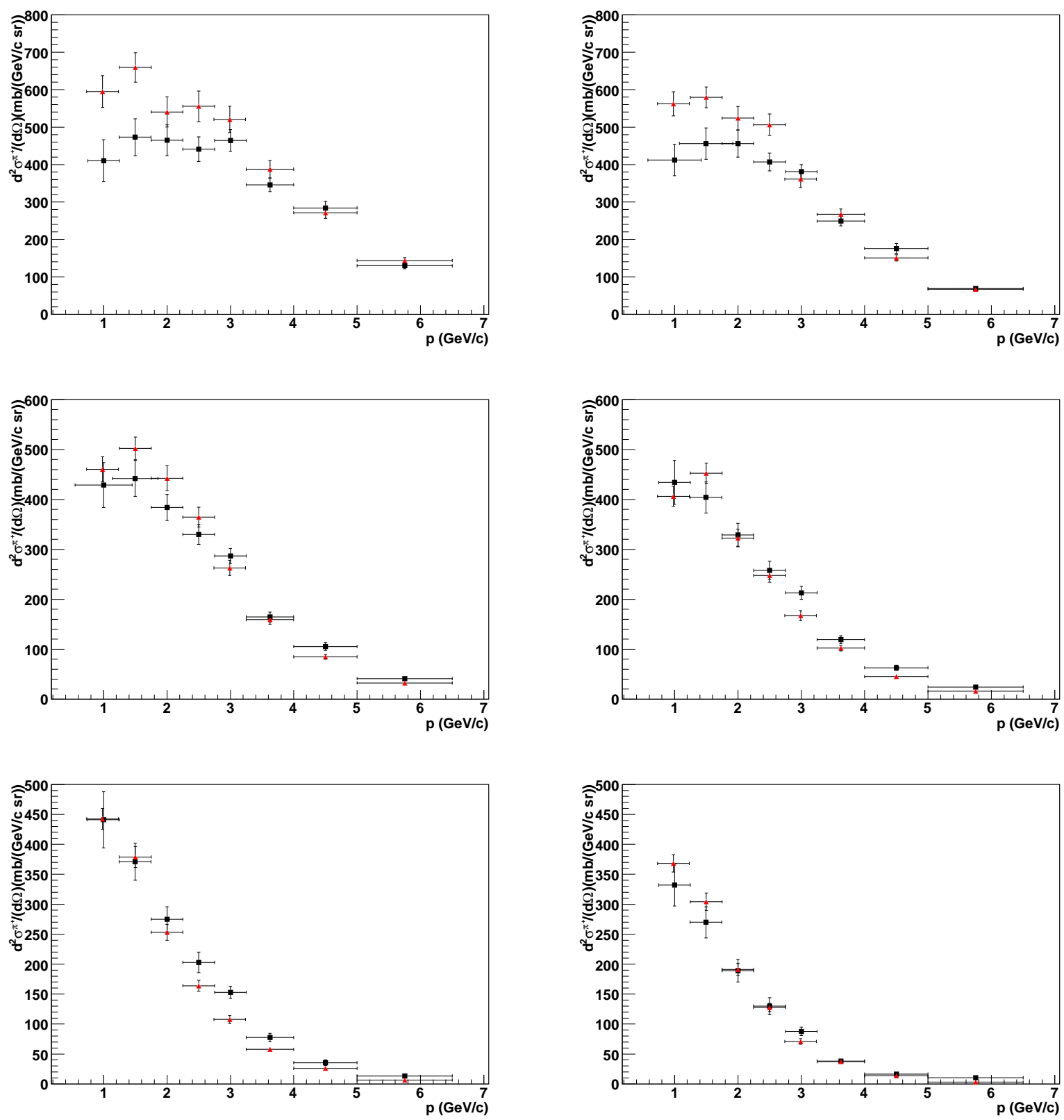

Figure 4.9: Measured double-differential $\pi^{+}$production cross-section $d^{2} \sigma /(d p d \Omega)$ for incoming protons of $12.9 \mathrm{GeV} / \mathrm{c}$ on an aluminium target as a function of pion momentum $\mathrm{p}$ for pion polar angle $30 \leq \theta<210 \mathrm{mrad}$. The results of MARS simulations are compared to the HARP experimental data. The $\mathrm{X}$ and $\mathrm{Y}$ axes are $\mathrm{GeV} / \mathrm{c}$ and $(\mathrm{mb} /(\mathrm{GeV} / \mathrm{c}$ sr)) respectively 

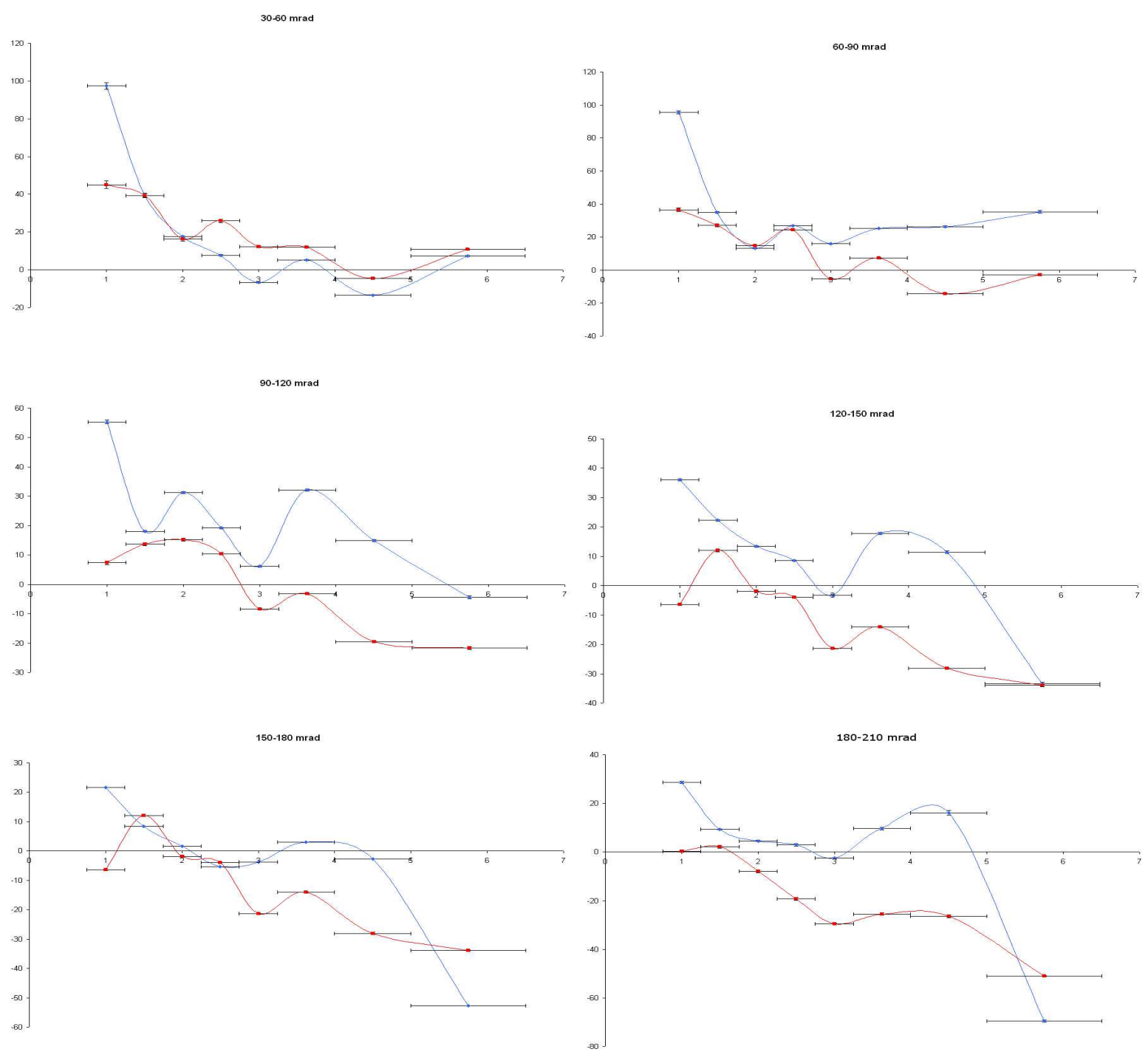

Figure 4.10: Percentage difference between the simulated results the HARP measured doubledifferential $\pi^{+}$production cross-section $d^{2} \sigma /(d p d \Omega)$ for incoming protons of $12.9 \mathrm{GeV} / \mathrm{c}$ on an aluminium target. The GEANT4-QGSC (blue) and and MARS15( red) simulations are compared. Y-axis shows ((Simulated Result-HARP Result)/(HARP result))*100 and X-axis shows total momentum of pion secondaries. Plots cover the whole 30-210 mrad angular range 


\subsection{Total Cross-sections}

The total cross sections were calculated as a function of total pion momentum and polar angle $\theta$. These results were calculated for the HARP data, all GEANT4 simulations and MARS simulations and are shown in Figures 4.11,4.12,4.13,4.14.

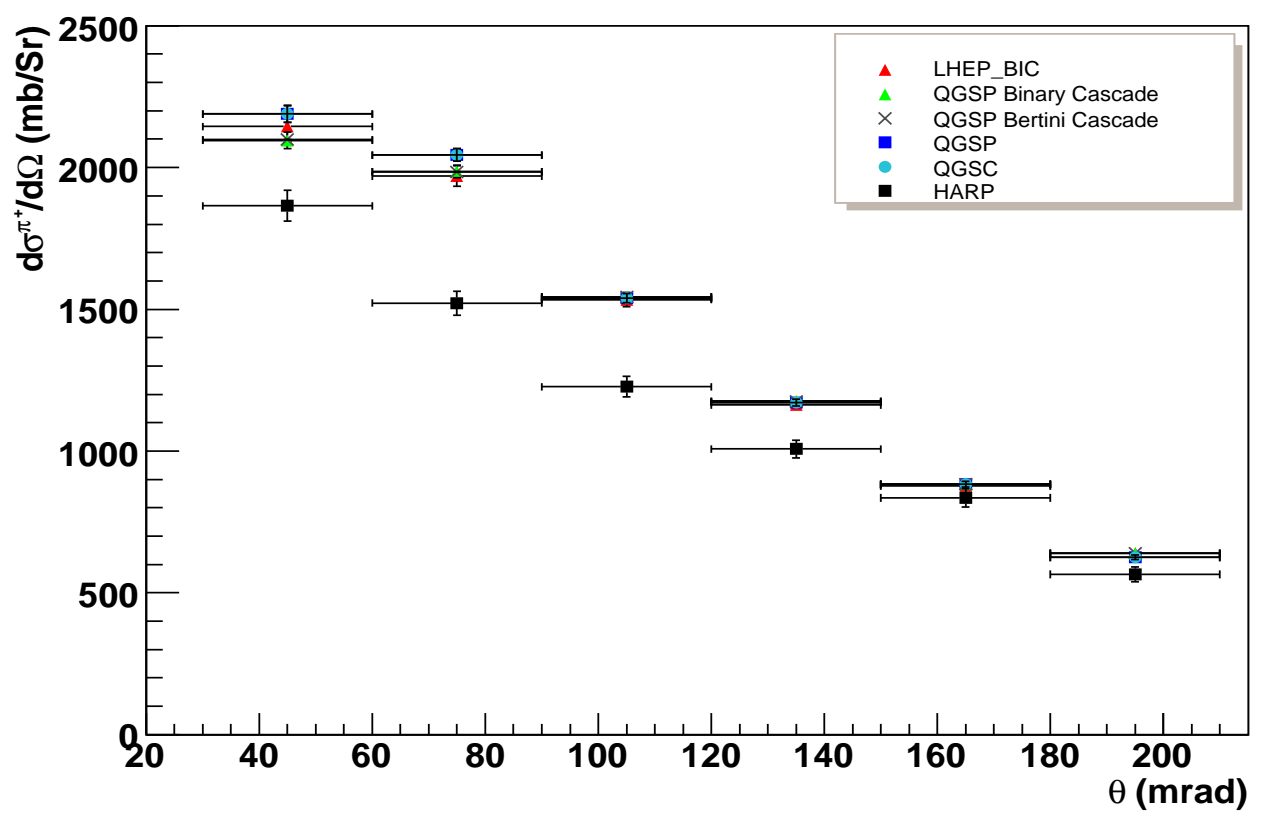

Figure 4.11: Measured differential $\pi^{+}$production cross-section $d \sigma / d p$ for incoming protons of $12.9 \mathrm{GeV} / \mathrm{c}$ on an aluminium target. The results of of GEANT4 simulations with different hadronic models are compared to the HARP experimental data

\subsection{Conclusions}

Firstly comparing GEANT4 hadronic engines. The results presented show that the GEANT4 hadronic models studied agree with each other at the level of a few percent with the exception of the Quark-Gluon-String models with chiral invariance(QGSC). QGSC performs much better in calculating $\pi^{+}$production cross-sections for pions with momenta less than $1.25 \mathrm{GeV} / \mathrm{c}$ across the whole angular range of the study (30-210 mrad). For pions with relatively high production angles (120-210) the models significantly underestimate pion production at a level of approximately 30$55 \%$.

It is evident that when comparing both codes that QGSC and MARS double-differential 


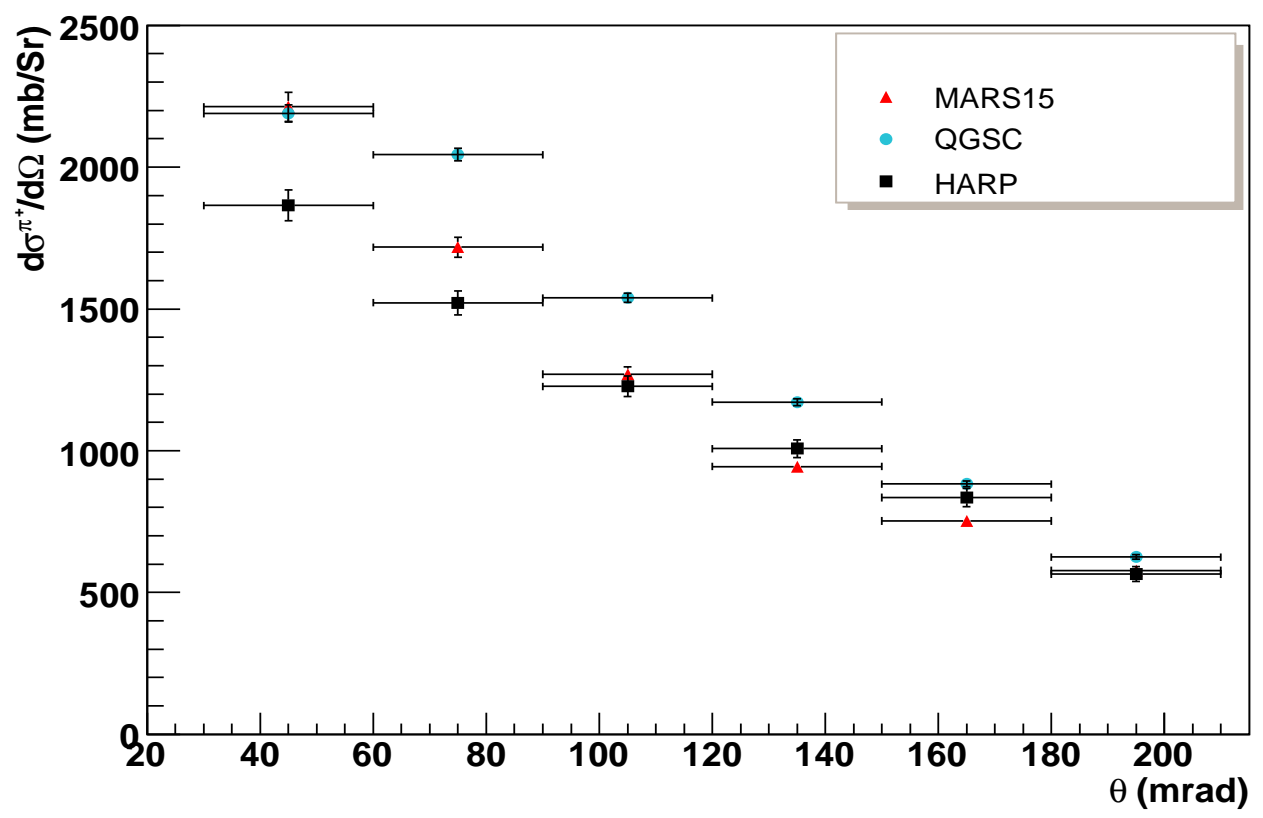

Figure 4.12: Measured differential $\pi^{+}$production cross-section $d \sigma / d p$ for incoming protons of $12.9 \mathrm{GeV} / \mathrm{c}$ on an aluminium target. The results of MARS simulations are compared to the HARP experimental data and GEANT4 QGSC model results

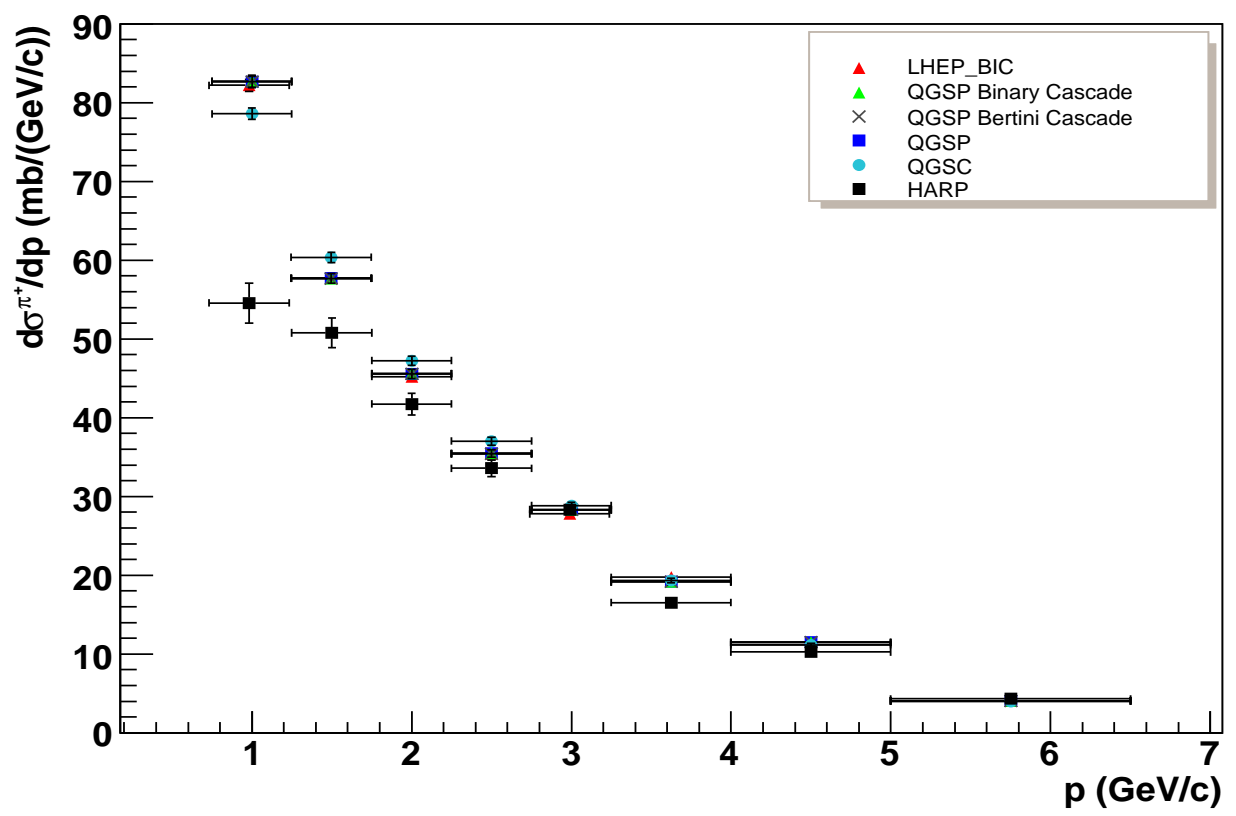

Figure 4.13: Measured differential $\pi^{+}$production cross-section $d \sigma / d \theta$ for incoming protons of $12.9 \mathrm{GeV} / \mathrm{c}$ on an aluminium target. The results of GEANT4 simulations with different hadronic models are compared to the HARP experimental data 


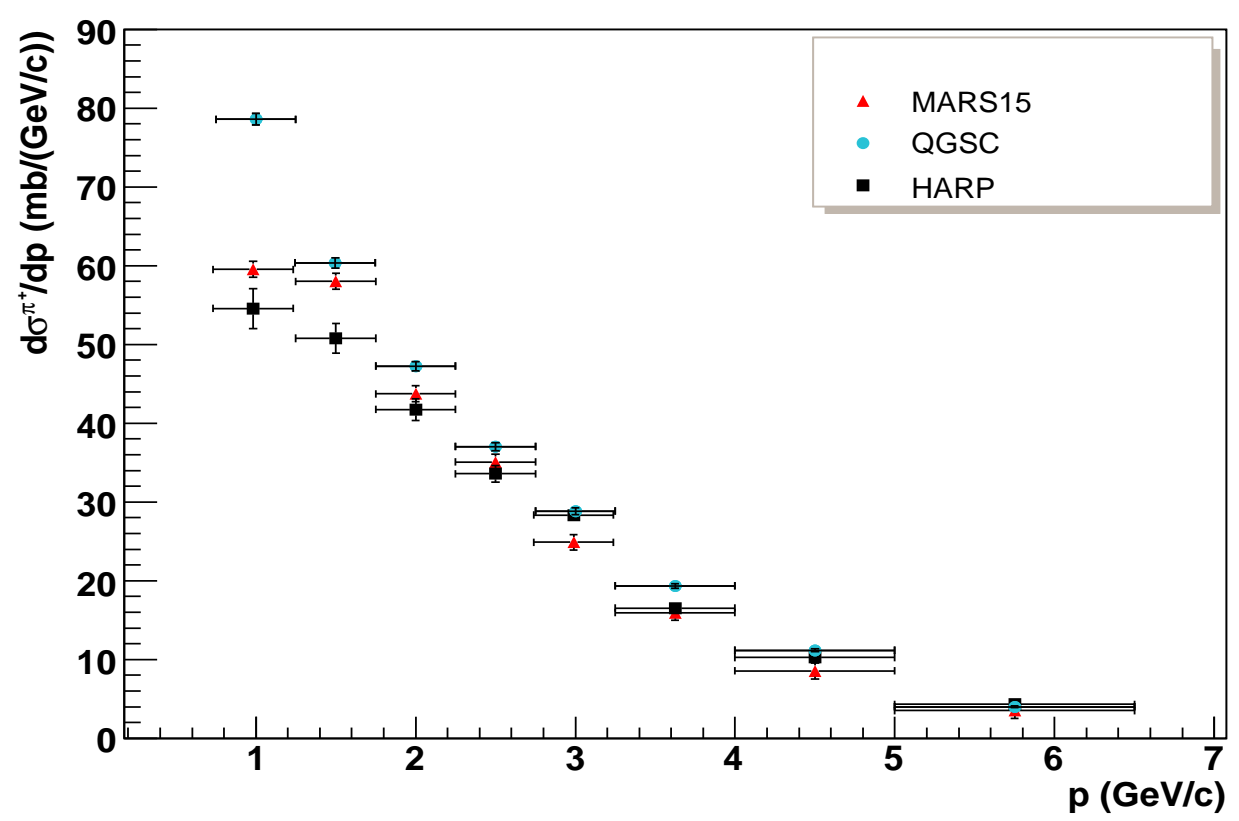

Figure 4.14: Measured differential $\pi^{+}$production cross-section $d \sigma / d \theta$ for incoming protons of $12.9 \mathrm{GeV} / \mathrm{c}$ on an aluminium target. The results of MARS simulations are compared to the HARP experimental data and GEANT4 QGSC model results

cross-sections deviate significantly from the HARP measured results at the extremities of our study range. Both codes overestimate pion production at low angles and low momenta where as at high angle, high momenta both codes heavily underestimate pion production. MARS performs better in both these extremes. In the mid momentum range both codes generally agree with the HARP measured cross-sections with an accuracy of between $+30 \%$ to $-30 \%$. Arguably the GEANT4 model performs slightly better than MARS in this region.

In terms of using these results as a bench-marking study to provide a weighting to the previous studies of pion yields in a Neutrino Factory it can be concluded that the MARS data give the more reliable results for $12.9 \mathrm{GeV} / \mathrm{c}$ protons on aluminium. For tracking through both the Chicane/Linac and Phase rotator channels detailed earlier, the region of momentum space that is successfully transported to the end of the channel is $50-300 \mathrm{MeV} / \mathrm{c}$ longitudinally and between $250-500 \mathrm{MeV} / \mathrm{c}$ transversely. These values fall below the lower limit of our study range although the trend seen in the low momentum results suggests that both codes will be significantly overestimating pion production in the transported momentum space. 


\section{Chapter 5}

\section{The Muon Ionisation Cooling Experiment (MICE)}

\subsection{Introduction}

A proof of principle experiment is being designed to investigate if ionisation cooling is a feasible technique to reduce the width and divergence of a muon beam. This is an essential requirement of a Neutrino Factory complex, as previously discussed, with the cooling channel providing up to an order of magnitude increase in intensity and accounting for up to $20 \%$ of the total cost of the facility. This experiment is called MICE (Muon Ionisaton Cooling Experiment).

On paper the principle of ionisation cooling looks theoretically sound. In reality, however, it is difficult to design an experiment to show this. An effective experiment involves development of many novel techniques and a fusion of accelerator development with particle physics detectors rarely seen. The requirements that MICE must show is that the experiment can reduce the beam transverse emittance by $>10 \%$ for muon momenta in the range $140 \rightarrow 240 \mathrm{MeV} / \mathrm{c}$. The measure of this emittance should be accurate to an absolute precision of $\pm 0.1 \%$.

The purpose of MICE is not purely to test muon ionisation cooling but to design, engineer and build a section of a Neutrino Factory Cooling channel. The experiment will be housed at the Rutherford Appleton Laboratory (RAL), Chilton, Oxfordshire in building R5.2. The proton source for MICE is the ISIS accelerator. ISIS is the world's most powerful pulsed neutron and muon source. The muons are produced from a secondary pion beam created by $800 \mathrm{MeV}$ 
protons interacting in a newly constructed titanium target. The beam is transported by a newly constructed beamline from the synchrotron vault to the MICE experiment. The beamline is a conventional pion-muon decay channel with optics manipulation for tunable beam preparation. The MICE beamline will be thoroughly described in Chapter 7 .

The muon beam will have its emittance evaluated before entering and after exiting the cooling channel (which will be described later in the chapter). The measurement precision required by MICE cannot be achieved by measuring beam envelopes hence the decision was made that MICE should be a single-particle experiment to allow for accurate and well defined kinematic measurements on a muon by muon basis. The emittance measurements will be made using two scintillating fibre $(\mathrm{SciFi})$ trackers. The trackers will determine the full 6-dimensional emittance by measuring the phase-space coordinates $\left[\mathrm{x}, P_{x}, \mathrm{y}, P_{y}, \mathrm{E}, \mathrm{t}\right]$. Correlations between these parameters are precisely the reason for carrying out single particle measurements. Providing that the statistics are high enough, these single particle measurements can be used to construct any input beam by appropriate weighting of particles. The effects of correlations are eliminated and it makes it easy to understand the time of the particle relative to the accelerating radio frequency $(\mathrm{RF})$ phase. Triggering of the experiment and coupling to the RF phase in addition to background rejection will be the responsibility of the particle identification (PID) detectors which, under the current baseline are 3 time-of-flight walls and a double aerogel Cherenkov device and a calorimeter for muon-electron separation.

As a result of funding constraints, the full MICE experiment will not be able to be completed until mid-2009. Construction of MICE will therefore be in stages with more adventurous physics goals becoming achievable as the stages progress. These stages can been seen in Figure 5.2. The first beam is scheduled to be available from the MICE beamline in April 2008. In addition the PID detectors, including a non-magnetised tracker, should be built and available at this time also. This is Stage I and it allows testing of detector data-acquisition, alignment of the tracker and determination of beam characteristics. The first measurement of emittance is achievable at Stage II with the inclusion of the first tracker solenoid. The addition of the second tracker and tracker solenoid constitutes Stage III. This allows comparison between both trackers and will be essential to measure systematic effects in each tracker. Stage IV is defined by the addition of the first absorber-focus-coil module and a measurement of muon ionisation can be made at this 


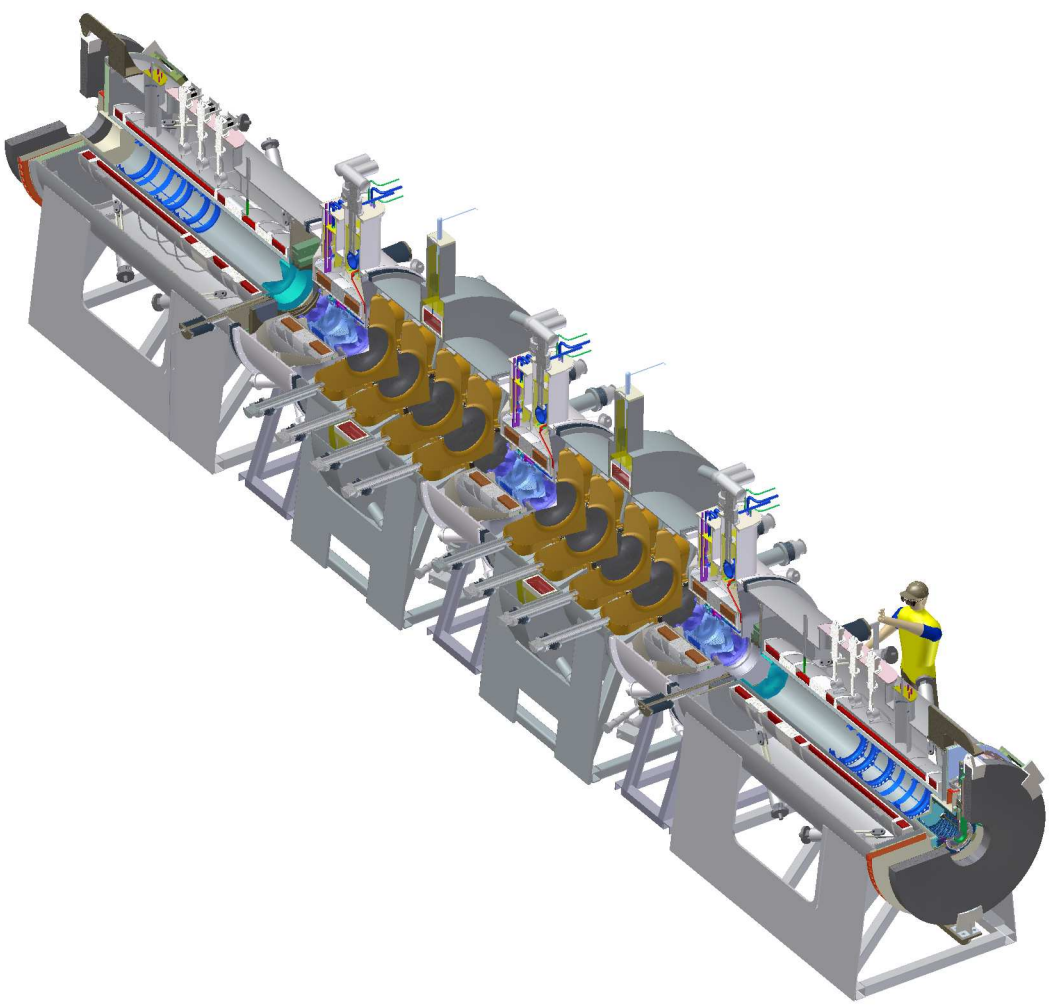

Figure 5.1: 3D layout of the full MICE Stage-VI experiment [67]

stage. The penultimate stage includes the addition of $4 \mathrm{RF}$ cavities and corresponding coupling coils. This will be useful to measure background from the RF cavities and to measure cooling with restoration of longitudinal momentum for the first time. Lastly, Stage VI is the full baseline MICE experiment with inclusion of the additional $4 \mathrm{RF}$ cavities, coupling coils and two further hydrogen absorbers with associated magnet elements (discussed in Section 5.8).

\subsection{Theory and definition of emittance}

The emittance of a particle beam is a measure of the parallelism of a beam. It gives a measure of the beam size and the ease of transporting the beam. By adopting the correct definition of emittance it can be used as a good way to predict beam propagation. This is because the beam 


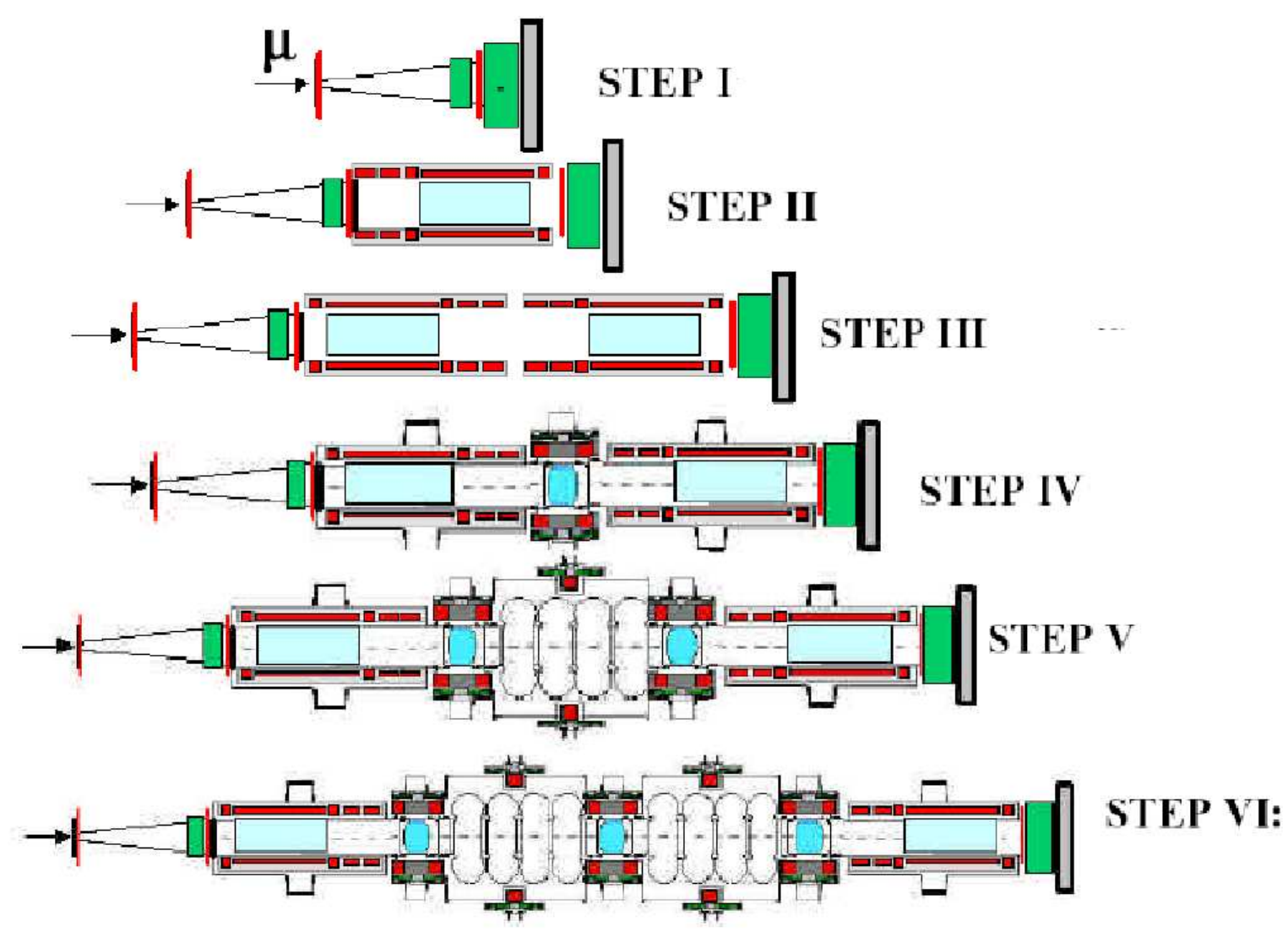

Figure 5.2: Planned staged evolution of the MICE experiment

emittance is a constant of motion if using the correct canonical coordinates of the Hamiltonian formalism.

In solenoidal optics one can deal with particle motion using a transfer map formalism [68]. In this scheme the particle evolution along the beam direction $\mathrm{z}$ is mediated via operators acting on the Hamiltonian. This method is extended to treatment of a particle bunch by dealing in moments of the phase space density function.

The phase-space of a beam of particles can be expressed in terms of six variables $\left[x, P_{x}, y, P_{y}, E, t\right]$. It is often more meaningful to work not in phase space but alternatively in trace-space. Trace-space is mapped from phase-space by using the relationship $P_{i} \rightarrow i^{\prime} . P_{z}$ where $\mathrm{i}=\mathrm{x}, \mathrm{y}$. Hence the trace-space vector is $\left[x, x^{\prime}, y, y^{\prime}, E, t\right]$ where $\mathrm{x}, \mathrm{y}$ are the transverse space coordinates, $x^{\prime}, y^{\prime}$ are the transverse divergences of the beam, $\mathrm{E}$ is energy and $\mathrm{t}$ is time.

Classically the beam emittance is zero as each particle occupies a zero volume in phase space. 
If one includes quantum fluctuations a beam will occupy a very small phase space volume. These two emittances are of no real practical interest. If one assumes that the beam measurement resolution is large compared to the average inter-particle spacing of the beam it is possible to derive a more meaningful definition of emittance. The beam can be modelled as a distribution function which has a non-negligible emittance. The volume occupied by this distribution in its 6-dimensional form is a constant and this is a consequence of the Liouville theorem which states that volumes of phase-space are invariant. If $\mathrm{G}(\mathrm{t})$ represents the state of a beam at time $\mathrm{t}$ with phase-space volume $\mathrm{V}$ and $\mathrm{G}\left(t^{\prime}\right)$ represents the state of the beam at time $t^{\prime}$ with volume $\mathrm{V}^{\prime}$ the Liouville theorem states that $\mathrm{V}=V^{\prime}[69]$. The hyperspace surface bounding the volume is not generally invariant. The form can be altered but the volume and hence the 6 -dimensional phase-space density are conserved.

The discussion of emittance conservation up to this point is not valid under acceleration. It is useful to introduce the concept of normalised emittance which is constant under acceleration.

$$
\epsilon_{n}=\beta_{k} \gamma_{k} \epsilon
$$

where $\beta_{k}$ and $\gamma_{k}$ are the standard kinematic Lorentz factors i.e. $\beta_{k}=v / c$ and $\gamma_{k}=\sqrt{1-\beta_{k}^{2}}$ One can define a matrix of covariances $(\mathrm{U})$ which is given in $2 \mathrm{D}$ by:

$$
U_{2 D}=\left(\begin{array}{cc}
\sigma_{11} & \sigma_{12} \\
\sigma_{21} & \sigma_{22}
\end{array}\right)=\left(\begin{array}{cc}
\sigma_{x x} & \sigma_{x x^{\prime}} \\
\sigma_{x x^{\prime}} & \sigma_{x^{\prime} x^{\prime}}
\end{array}\right)
$$

This matrix contains information on the moments of the beam parameters and it can be related to the lattice as follows:

$$
U_{2 D}=\epsilon_{x}\left(\begin{array}{cc}
\beta & -\alpha \\
-\alpha & \gamma
\end{array}\right)
$$

where $\alpha, \beta, \gamma$ are the known as the Twiss parameters of the lattice, and $\epsilon_{x}$ is the emittance in the $\mathrm{x}$-direction.

The Twiss parameters are a result of a general parameterisation of a transfer matrix which effectively propagates the Hill's equations expressing the linearised betatron motion [70]. These parameters, expressed in a natural form, are given by (5.2), (5.3), (5.4) and give respectively 
the variance of the beam in configuration space, variance of momentum (or equivalently divergence) of the beam in momentum (trace) space and the covariance between the variances in configuration and trace spaces.

$$
\begin{gathered}
\left\langle x^{2}\right\rangle=\beta_{x} \epsilon_{x} \\
\left\langle P_{x}^{2}\right\rangle=\gamma_{x} \epsilon_{x} \\
\left\langle x P_{x}\right\rangle=-\alpha_{x} \epsilon_{x}
\end{gathered}
$$

Emittances can be defined in a number of dimensions and hence the corresponding hyperellipsoids. One can talk about emittances in $2 \mathrm{D}\left[\left(x, x^{\prime}\right),\left(y, y^{\prime}\right),(\mathrm{E}, \mathrm{t})\right], 4 \mathrm{D}\left[\left(x, x^{\prime}, y, y^{\prime}\right),\left(x, x^{\prime}, \mathrm{E}, \mathrm{t}\right)\right.$, $\left.\left(y, y^{\prime}, \mathrm{E}, \mathrm{t}\right)\right]$ or $6 \mathrm{D}\left[x, x^{\prime}, y, y^{\prime}, E, t\right]$.

The 4D normalised transverse emittance can be defined in phase space as:

$$
\epsilon_{n, r m s_{\perp}}=\frac{1}{m_{\mu} c} \sqrt[4]{\left|U_{4 D}\right|}
$$

With the normalised, longitudinal emittance given by (5.6):

$$
\epsilon_{n, r m s_{(z, P z)}}=\frac{1}{m_{\mu} c} \sqrt{\left|U_{2 D}\right|}
$$

Similarly in trace-space the transverse, normalised emittance is given by (5.7):

$$
\epsilon_{n, r m s}=\frac{\bar{p}_{z}}{m_{\mu} c} \sqrt[4]{\left|U_{4 D}\right|}
$$

Commonly in accelerator physics the emittance of a distribution is expressed as an ellipsoid in phase/trace space. An example of a trace space ellipse in $2 \mathrm{D}$ can be seen in Figure 5.3. The area of this ellipse is Liouville invariant and geometrically is given by (5.8)

$$
\text { Area of an ellipse }=\pi a b=\pi(\epsilon \beta)^{1 / 2} \cdot(\epsilon / \beta)^{1 / 2}=\pi \epsilon
$$

And following the discussion stated previously the area of this ellipse can be expressed as: 


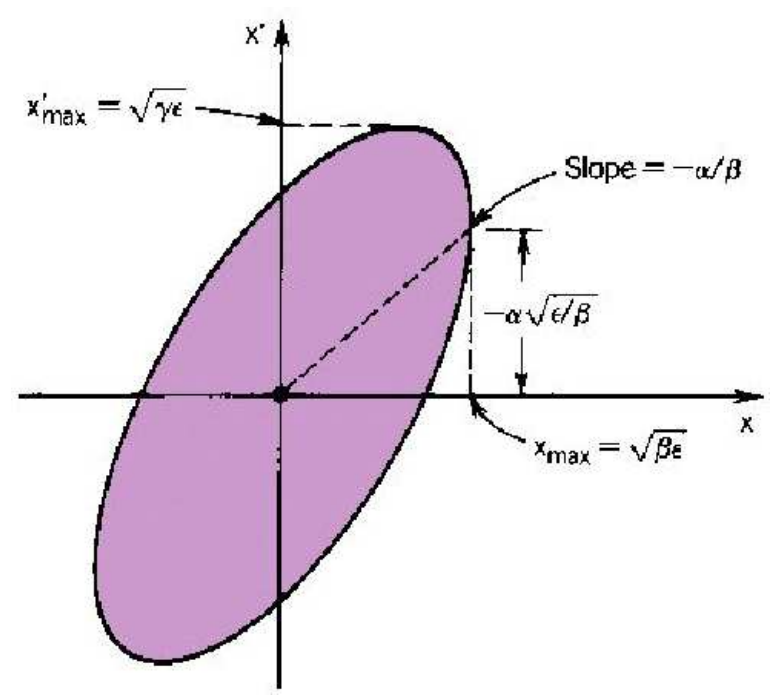

Figure 5.3: An example of a typical trace-space ellipse [69]

$$
A=\pi \sqrt{|U|}
$$

\subsection{Muon Cooling}

As previously discussed, the muons produced in a Neutrino Factory from pion decay after the target occupy a phase space volume too large to inject into a conventional accelerator. The solution to this problem is to reduce the transverse emittance of the beam. Familiar methods of cooling such as stochastic, electron and laser cooling are not feasible when it comes to the design of a Neutrino Factory. This is because for effective cooling these methods take of the order a few seconds to minutes, this is too slow to cool muons which decay in $2.2 \mu s$ on average in their rest frame. In the longer term ionisation cooling is also essential for a Muon Collider complex. The muon collider will probe electroweak symmetry breaking at the TeV energy scale [71]. The luminosity determines the scope of the physics potential of a collider experiment. To increase the luminosity to the level required to fulfil the physics goals, the emittances of the beams will have to be reduced considerably.

To achieve this essential transverse cooling for a Neutrino Factory and a Muon Collider we must take a novel approach, muon ionisation cooling. As the name suggests muon ionisation 
cooling is cooling due to ionisation losses in material. As the muon traverses matter it loses energy, its momentum is reduced in both the transverse and longitudinal components. Ionisation in the material decreases muon momentum while, to first order, keeping the beam size constant. The reduction in energy of the muons is obviously not desirable and this energy must be replaced. This is done using RF cavities, which accelerate the muons and replace the longitudinal component of momentum [72]. It is not all good news however, the cooling effect is tempered by multiple scattering in the ionising material.

To derive an expression for the reduction of normalised emittance [73] one notes that from equation (5.1) the definition of normalised emittance is given by $\epsilon_{n}=\beta_{k} \gamma_{k} \epsilon$. Differentiating this expression gives (5.10):

$$
\frac{d \epsilon_{n}}{d z}=\epsilon \frac{d\left(\beta_{k} \gamma_{k}\right)}{d z}+\frac{d \epsilon}{d z}\left(\beta_{k} \gamma_{k}\right)
$$

By differentiation the expression of the determinant of the moment matrix in two dimensional trace-space one finds (5.11)

$$
2 \epsilon \frac{d \epsilon}{d z}=\frac{d \epsilon^{2}}{d z}=\sigma_{x x} \frac{d \sigma_{x^{\prime} x^{\prime}}}{d z}+\sigma_{x^{\prime} x^{\prime}} \frac{d \sigma_{x x}}{d z}-2 \sigma_{x x^{\prime}} \frac{d \sigma_{x x^{\prime}}}{d z}
$$

The differential change in beam moments are given by (5.12) [74]:

$$
\frac{d \sigma_{x x}}{d z}=2 \sigma_{x x^{\prime}} \quad \frac{d \sigma_{x x^{\prime}}}{d z}=\sigma_{x^{\prime} x^{\prime}}-a \sigma_{x x} \quad \frac{d \sigma_{x^{\prime} x^{\prime}}}{d z}=\sigma_{x^{\prime} x^{\prime}}-2 a \sigma_{x x^{\prime}}
$$

Substitution of (5.13), (5.12) in (5.11) gives (5.14):

$$
\begin{gathered}
\frac{d \beta_{k} \gamma_{k}}{d z}=\frac{1}{\beta_{k} m c^{2}} \frac{d E}{d z} \\
\beta_{k} \gamma_{k} \frac{d \epsilon}{d z}=\frac{\beta_{k} \gamma_{k}}{2 \epsilon}\left(\sigma_{x x}\left(\sigma_{x^{\prime} x^{\prime}}-2 a \sigma_{x x^{\prime}}\right)+\sigma_{x^{\prime} x^{\prime}}\left(2 \sigma_{x x^{\prime}}\right)-2 \sigma_{x x^{\prime}}\left(\sigma_{x^{\prime} x^{\prime}}-a \sigma_{x x}\right)\right)
\end{gathered}
$$

where $\mathrm{a}$ is a constant. 
After appropriate cancellation and noting from the expression of the covariance matrix in terms of Twiss parameters that $\sigma_{x x}=\epsilon \beta$ leaves one remaining term such that:

$$
\beta_{k} \gamma_{k} \frac{d \epsilon}{d z}=\frac{1}{2} \beta_{k} \gamma_{k}\left(\beta \sigma_{\theta}^{2}\right)
$$

where multiple scattering is given by [75]

$$
\sigma_{\theta}^{2}=\left(\frac{13.6[M e V]}{p \beta_{k} c}\right)^{2} \frac{z}{X_{0}}
$$

This finally gives the expression for cooling relevant to the MICE experiment [73]

$$
\frac{d \varepsilon_{n}}{d s}=-\frac{1}{\beta_{k}^{2}}\left|\frac{d E_{\mu}}{d s}\right| \frac{\varepsilon_{n}}{E_{\mu}}+\frac{1}{\beta_{k}^{3}} \frac{\beta_{\perp} 0.014^{2}}{2 E_{\mu} m_{\mu} X_{0}}
$$

where $\epsilon_{n}$ is the normalised emittance, $\beta_{k}, \gamma_{k}$ are the usual relativistic factors, $X_{0}$ is the

radiation length of the absorbing medium, $\left|\frac{d E_{\mu}}{d s}\right|$ is the average rate of change of energy and $\beta_{\perp}$ is the transverse beta function (twiss parameter).

The first cooling term is due to ionization and the second heating term is due to multiple Coulomb scattering. From the equation above it can be seen that for fast cooling of the beam to low emittance we require:

1. Low $\beta_{\perp}$ (i.e. strong focusing) $\rightarrow$ Superconducting Solenoids

2. Absorber with long radiation length (i.e. a low $\mathrm{Z}) \rightarrow$ liquid $H_{2}$

3. High gradient conducting RF cavities

\subsection{Simulation}

Simulations of the MICE experiment are essential for design and preparatory work preconstruction for the experiment. Physics goals must also be studied including estimations of background and detailed design of cooling measurements. Essential calculations of electromagnetic fields along the MICE channel must be studied as must their effects on sensitive components of the experiment such as PID detectors. This is carried out by the G4MICE software package. 
G4MICE [76] is a software package written in $\mathrm{C}++$ which carries out the following duties: Monte Carlo simulation of the passage of particles through matter and fields; Simulation of the response of the detectors to these particles including detector geometries, resolutions, thresholds, electronic responses; Reconstruction of space-points and then tracks from the raw detector data; Analysis of reconstructed particle data to calculate emittances etc.

The G4MICE package incorporates many well established external packages to add functionality. The Monte Carlo tracking and the majority of the physical processes are inherited from the GEANT4 toolkit [51] which is the standard HEP physics simulation package. Descriptions of magnetic elements, cavities and absorbers are derived from the Fermilab BeamTools package. Mathematical functionality is acquired via the GSL (GNU scientific library) and CLHEP (HEP utilities). The detailed calculation of optimal track parameters uses the Kalman filter package citekalman and finally data analysis libraries are inherited from the ROOT HEP analysis toolkit [66]. A key feature of G4MICE which currently does not exist but will be required to achieve the full physics goals of the experiment is the creation of virtual bunches in the software. This will enable the benchmarking of cooling performance for a large number of Neutrino Factory front end beams.

\subsection{Particle Detection and Kinematic Measurement}

The particle detectors alluded to in the previous section are primarily designed to accurately measure the volume occupied by the muon in $6 \mathrm{D}$ phase space as well as count the number of particles. The decision was made to make measurements particle-by-particle rather than use a multi-particle approach. This decision was taken as it would have been very difficult to fully determine the $6 \mathrm{D}$ covariance matrix using a multi-particle approach as it would involve developing specific diagnostics unlike those commonly used in particle physics. Restricting the experiment to having only one particle traveling through per system time window is challenging in itself and involves careful design along the entire channel. It does however bring advantages such as easy investigation of correlations between particle $6 \mathrm{D}$ parameters and makes it easy to derive different input beam conditions from a single data set. A more detailed account of the detector systems that will enable these measurements can be found in the MICE Technical Reference Document (TRD) [77]. The individual MICE sub-systems will be briefly described in 
the remainder of this chapter with the reader pointed to [77] for additional detail.

\subsubsection{Time-of-flight Detectors}

The MICE experiment will have three fast Time-of-Flight (TOF0, 1, 2) scintillators [78]. These scintillators will be fast with a time resolution of roughly $70 \mathrm{ps}$. The first two of these known as TOF0 and TOF1 are placed upstream of the cooling channel, the actual separation of the TOF stations has not been finalised as they are a key component of beamline optics tunes. However, the key constraint is that they should be placed approximately $10 \mathrm{~m}$ from each other.

Each TOF station has a square active area of $48 \times 48 \mathrm{~cm}^{2}$ transverse to the beam and consists of two planes each measuring 1" thick. In TOF1\&2 the planes are constructed from 8 slabs of scintillator with dimensions $48 \times 6 \times 2.5 \mathrm{~cm}^{2}$ arranged perpendicular to each other. TOF0 is constructed similarly but with 12 slabs of $48 \times 4 \times 2.5 \mathrm{~cm}^{2}$. The slabs are comprised of Bicron BC404 scintillator which has intrinsic properties of $1.6 \mathrm{~m}$ bulk light attenuation length and a decay constant of $1.8 \mathrm{~ns}$. Light is collected from each end of every scintillator bar and transported to photomultiplier tubes (PMTs) via lightguides. TOF0\&1 are the furthest upstream and both need to operate in high rate environments. The TOF0 rate is particularly high. Additionally TOF1\&2 are located in high magnetic field regions. This has prompted the decision to use Hamamatsu R4998 photomultipliers for TOF0 and Hamamatsu R4998 photomultipliers for TOFs 1 and 2. These are fast photomultipliers which are also insensitive to magnetic fields. The specifications of both these tubes can be seen in Table 5.5.1.

\begin{tabular}{c|c|c} 
Characteristic & R4998 (H6553 assembly) & R7761 (H8490 assembly) \\
\hline \hline Transit-time jitter & $160 \mathrm{ps}$ & $360 \mathrm{ps}$ \\
Anode pulse rise-time & $0.7 \mathrm{~ns}$ & $2.1 \mathrm{~ns}$ \\
Gain $(\mathrm{B}=0 \mathrm{~T})$ & $5.7 \times 10^{6}$ & $1.0 \times 10^{7}$ \\
Gain $(\mathrm{B}=0.5 \mathrm{~T})$ & $\mathrm{N} / \mathrm{A}$ & $3.0 \times 10^{6}$ \\
Useful diameter & $20 \mathrm{~mm}$ & $27 \mathrm{~mm}$ \\
Maximum output current & $16 \mu \mathrm{A}$ & $10 \mu \mathrm{A}$ \\
Max allowed rate & $267 \mathrm{KHz}$ & $167 \mathrm{KHz}$
\end{tabular}

The combination of TOF stations will provide muon identification. A coincidence with TOF2 will identify the muons that travel the complete length of the cooling channel and therefore the ones that should be studied to show cooling. In addition, a coincidence in the two most upstream 
TOF walls will act as a DAQ trigger for the rest of the experiment.

\subsubsection{Tracking Detectors}

The purpose of the tracking detectors are to measure all muon parameters upstream before cooling and again downstream post-cooling. Both spectrometers are identical, with each providing a high resolution measurement of the muon helix in a $4 \mathrm{~T}$ solenoidal magnetic field at a high precision time measurement. The superconducting solenoid surrounding the tracker has a $40 \mathrm{~cm}$ diameter inner bore and produces a $4 \mathrm{~T}$ field which is constant to $1 \%$ over a $1 \mathrm{~m}$ long region.

The baseline option for the tracking detector are two scintillating fibre trackers. Each Sci-Fi consists of five planes of three doublet layers at $120^{\circ}$ to each other. To reduce multiple scattering in the tracker itself the material content of the beam was kept to minimum, hence the fibres chosen were $350 \mu \mathrm{m}$ Kuraray doped polystyrene fibres. The doping chemical used is $3 \mathrm{HF}$ dye (3-Hydroxy Flavone) which has a typical emission wavelength of $\approx 520 \mathrm{~nm}$ and is the typical wavelength where the optical and electronics (VLPC) set-up has the highest sensitivity. The light from each fibre will be transferred to photon detectors via clear fibres. Due to the relatively low light yield from the fibres the photon detection scheme chosen was the VLPC (Visible Light Photon Counters) hardware developed for the D0 experiment at Fermilab [79]. The VLPC system is ideally suited for this application having a high quantum efficiency of $\approx 80 \%$ and a gain of the order of 50,000. The system is robust having been operated successfully at D0 for 4 years. Each VLPC readout cassette consists of 1024 channels held cryogenically at $6 \mathrm{~K}$ using a liquid helium flow. Readout from the VLPC cassettes is carried out by analogue front-end (AFE) boards, again developed for D0. These boards amplify and digitise the signal from the VLPCs and also provide data reduction by zero suppression. Data aquisition is performed by interface to a VME crate which is then subsequently interfaced to a PC.

This tracker configuration gives effective space-point reconstruction since hits in two out of the three doublets will be adequate. A property of this design is that it will reduce the effect of background photons since a photon is unlikely to produce a hit in all three doublets in a fibre plane. The Sci-Fi has the advantages that it can operate at very high background rates which is an environment that it may find itself being situated so close to the RF cavities, it is immune to electromagnetic interference and it is totally passive. Simulations of the performance of the 
tracker have been studied using G4MICE. Figure 5.4 shows the $P_{T}$ and $P_{Z}$ resolution obtained from those simulations.
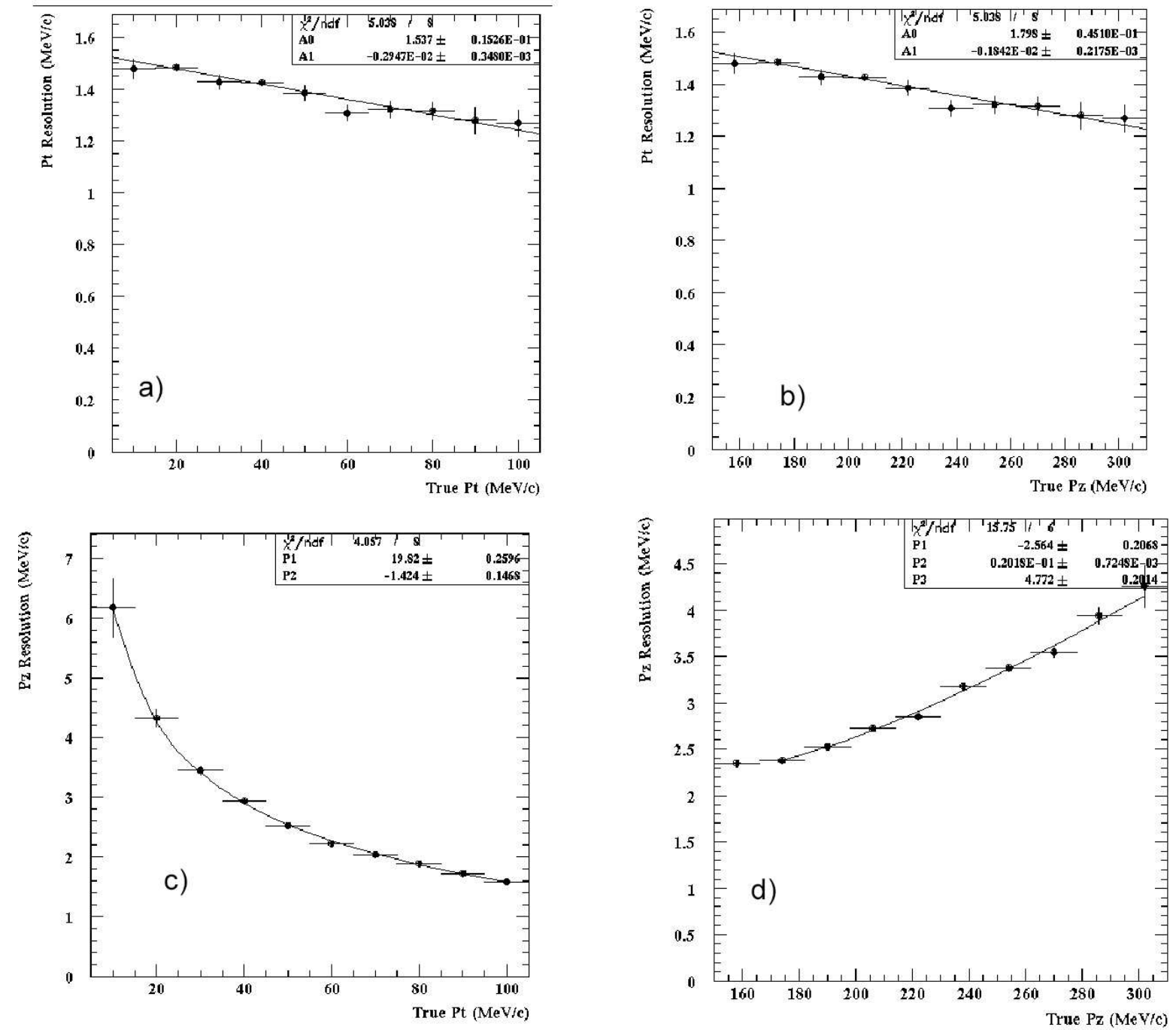

Figure 5.4: a) Resolution of $P_{T}$ as a function of $P_{T}$ b) Resolution of $P_{T}$ as a function of $P_{z}$ c) Resolution of $P_{z}$ as a function of $P_{T}$ d) Resolution of $P_{z}$ as a function of $P_{z}$ [80]

\subsubsection{Downstream Electron-Muon Separation}

With the muon lifetime being relatively short $(2.2 \mu \mathrm{s})$ there is the possibility that a muon could decay as it travels along the cooling channel or indeed in one of the spectrometers. This will affect $1 \%$ of events. The decay electrons will modify the emittance measurement considerably and therefore it is essential that they be identified and then rejected.

Kinematic constraints can be used to cut around $80 \%$ of these electrons, however, the pres- 
ence of any electrons are so detrimental to emittance measurement that even the remaining $20 \%$ will have significant effect. An electromagnetic calorimeter will be constructed and placed in the experiment to eliminate the remaining decay electrons. The electromagnetic calorimeter is based on the calorimeter previously designed for KLOE [81]. The design involves glueing 1mm diameter scintillating fibres between $0.3 \mathrm{~mm}$ shaped lead layers. This lead layer also adds strength to the structure. The fibre choice was Pol.Hi.Tech. type 044 which has an attenuation length of $\approx$ $3.5 \mathrm{~m}$ and decay constant of $2.5 \mathrm{~ns}$. The dimensions of the EMCAL is $120 \times 120 \mathrm{~cm}^{2}$ transverse to the beam and $16 \mathrm{~cm}$ thick (corresponding to 180 fibre-lead sandwiches). Lightguides couple to 1-1/8" R1355 Hamamatsu phototubes. The light collected by the phototubes will be digitised by the front-end electronics. A VME based system will be utilised with CAEN 792 ADCs.

As previously stated, the purpose of the downstream particle ID detectors is e- $\mu$ separation. In the calorimeter the signal deposited by a minimum ionising particle (mip) in each calorimeter cell is equivalent to that of a $27 \mathrm{MeV}$ electron or photon. In MICE the momentum of the muons produced will cause it to penetrate through all the calorimeter layers. On the other hand, electrons produced will leave most of their energy in the first two layers of the calorimeter. It is this property that will enable electrons and muons to be distinguished. Measurement of deposited energy and longitudinal energy deposition profile in the calorimeter should provide electron rejection of $10^{-3}$.

\subsubsection{Cherenkov Detectors}

The majority of pions transported along the MICE beamline decay to muons. However there still exists a background of pions and decay electrons. The inclusion in the beamline of an upstream Cherenkov detector aims to reduce this background significantly and provide a pure muon beam to the MICE experiment. The original detector design consisted of four $200 \mathrm{~mm}$ photomultipier tubes each surrounding a $22 \mathrm{~mm}$ thick, $20 \mathrm{~mm} C_{6} F_{14}$ liquid fluorocarbon radiator section. The threshold levels for this device are $140 \mathrm{MeV} / \mathrm{c}$ for muons and $190 \mathrm{MeV} / \mathrm{c}$ for pions and together this provides sufficient rejection.

A recent modification to the baseline Cherenkov detector has led to a redesign of the detector and involves the use of two Cherenkov radiators placed one after the other. The radiators for the detectors have refractive indices of 1.07 (unit 1) and 1.12 (unit 2) respectively. This configuration 
allows clean determination of particle type through the following logic. For incident muons with momenta in the range $207<\mathrm{P}<276 \mathrm{MeV} / \mathrm{c}$ only unit 1 will be above threshold and hence produce a signal. This has been calculated theoretically and can been seen in Figure 5.5. For muons in the momentum range $276<\mathrm{P}<365 \mathrm{MeV} / \mathrm{c}$ both units 1 and 2 will show coincident signals for muons only. For higher momenta muons both units will fire however this is not foreseen as a problem as it is known that incident pion momenta will not exceed $350 \mathrm{MeV} / \mathrm{c}$.

Each square detector unit measures $786 \mathrm{~mm}$ transverse to the beam and the structure is constructed from standard $15 \mathrm{~mm}$ steel plates. An optical glass window is positioned before the radiator block itself. The baseline radiator choices are Matsushita aerogel radiators with refractive indices of 1.07 and 1.12 as previously discussed. The light produced in each radiator is transported to 4 identical photomultiplier tubes fixed at the centre of each side of the box transversely to the beam via reflection from conical mirrors. These mirrors have a relatively complex shape to transport light efficiently. They are made from 3-mm thick polycarbonate sheets covered with a reflective layer of Aluminium, Silicon Oxyde $\left(\mathrm{SiO}_{2}\right)$ and Hafnium Oxyde. The photomultiplier tubes chosen are EM19356KA. These tubes have an effective diameter of $200 \mathrm{~mm}$ and have the advantage of being low noise tubes.

The two unit Cherenkov detector in MICE is placed between both TOF0 and TOF1. The key aim of this detector is to provide separation of muons, pions and electron of the same momentum by the differences in their Cherenkov light yield as a function of momentum. This Cherenkov detector will act to suppress any background from pions and electrons that are missed by the TOF detectors.

\subsection{Absorbers}

The reduction of normalised emittance in the MICE experiment is achieved in three liquid hydrogen absorbers. Liquid hydrogen was chosen as the absorber material due to its large ionisation energy loss rate and small probability of multiple scattering. Two other possibilities which will achieve slightly less cooling but are more practical than liquid hydrogen are solid lithium hydride $(\mathrm{LiH})$ or beryllium $(\mathrm{Be})$. The key criteria that the construction of these absorbers must satisfy are that they must 1) minimise multiple scattering, 2) achieve sufficient heat transfer rates such that the temperature and density of the absorbers remain uniform, and, 3) safe 


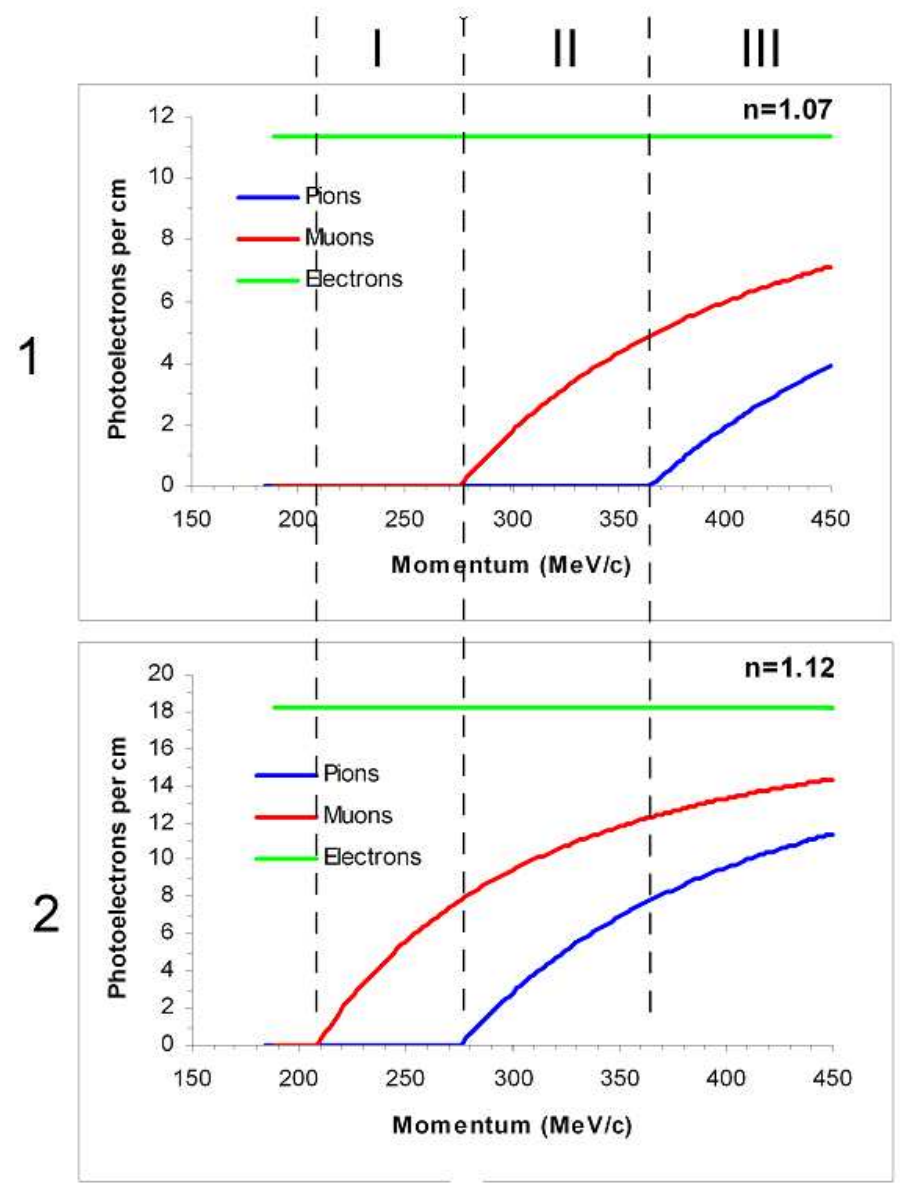

Figure 5.5: Scintillation light thresholds for both aerogel Cherenkov devices. The numbered regions indicate changes in triggering behaviour. Region I one unit triggers, region II both units trigger for muons only, region III both units trigger.

operation. The hydrogen absorber and focusing solenoid are designed to be mounted as a single module known as the Absorber Focusing Coil Module (AFC). The absorber vessel itself is made from aluminium and is $350 \mathrm{~mm}$ long at its centre with the beam windows being $300 \mathrm{~mm}$ diameter and also made from aluminium. This container will hold 21 litres of hydrogen which has an effective cooling mass of $18.3 \mathrm{~kg}$. The hydrogen will be kept in an artificial environment with the temperature and pressure tolerances being $15-21 \mathrm{~K}$ and $0.14-1.6$ bar respectively. This will be maintained by natural convection from a Cryo-cooler. 


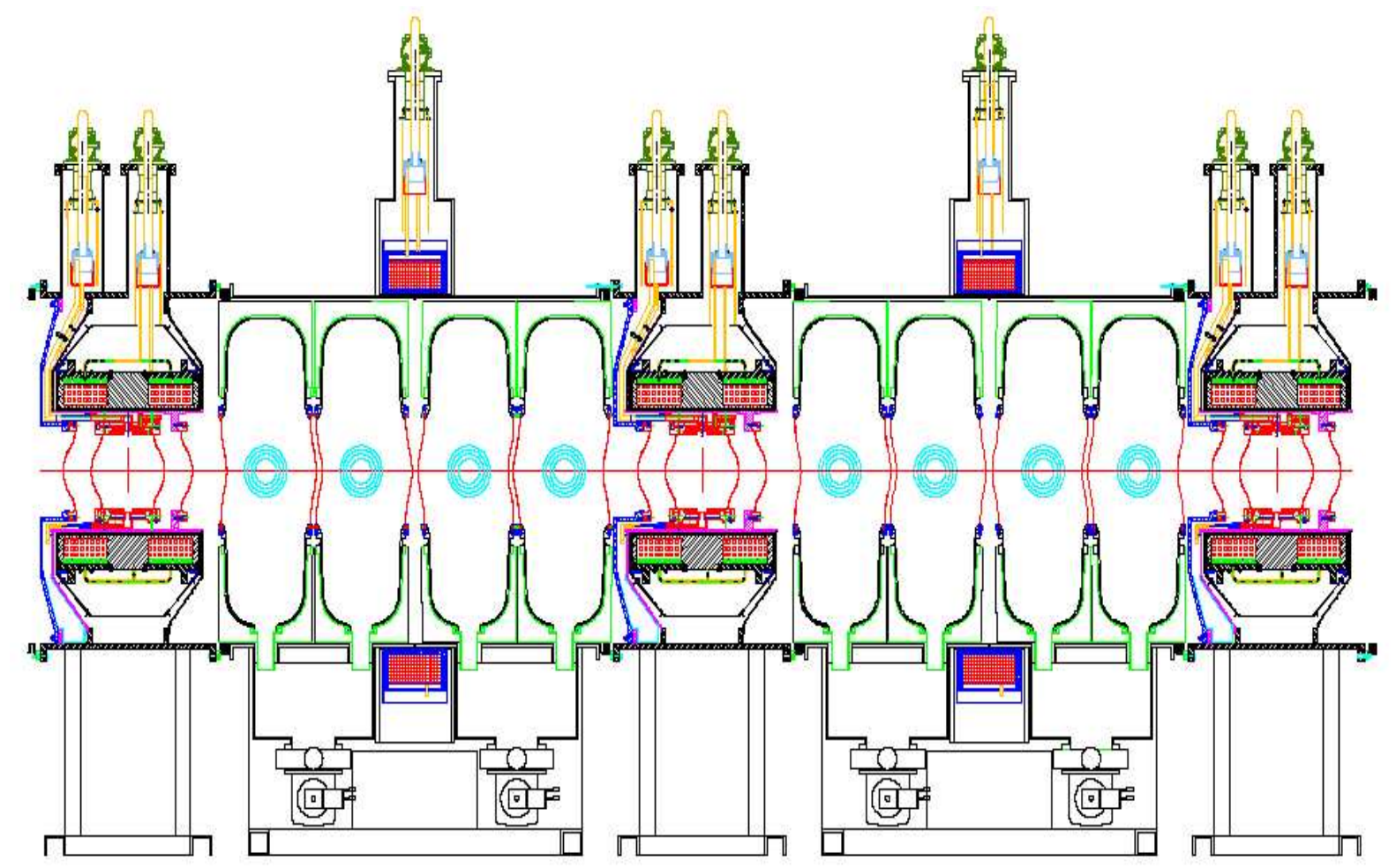

Figure 5.6: Engineering drawing of the RF cavity and absorber layout for the full MICE Stage VI. The eight RF cavities can be seen with curved terminating beryllium windows and coupling coils. The three absorber-focus-coil modules can clearly be seen. All superconducting magnetic elements are shown in red.

\subsection{RF Cavities}

The purpose of the RF cavities in the MICE experiment are to reinstate the longitudinal component of momentum that is lost in the absorbers. The cavity system is composed of eight 201.25 MHz RF cavities in two 4-cavity groups. The cavities are based on those of the Neutrino Factory MuCool Study II cooling channel [41]. The MICE cavities will have a maximum operating gradient of $\approx 8 \mathrm{MV} / \mathrm{m}$. This gradient is approximately half that studied in [41] and is a consequence of financial limitations on the power available to drive the cavities. The cavities will need to operate in a high B-field region and hence are required to be normally conducting. The $\mu^{ \pm}$beam will pass through large apertures electromagnetically sealed using curved beryllium windows. This cavity scheme should allow the MICE experiment to achieve a maximum accelerating voltage of $\approx 20 \mathrm{MV}$.

Some relevant issues for the downstream particle detectors concerning the cavities is the background they produce. The dark current (low energy electrons) produced on the inner 
surface of the cavities by field emissions are accelerated in the cavity and produce bremsstrahlung photons when they hit material. These effects are currently being simulated.

\subsection{MICE Magnetic Channel}

The MICE channel contains 18 superconducting solenoids. These magnets are used to transport and contain the muon beam as it passes through MICE. It is very important that the optics of this channel be well understood as it can have a major effect on the physics goals of the experiment. The magnet assemblies come in three main categories:

- Absorber focus coils

- RF coupling coils

- Tracker solenoids

Each hydrogen absorber will be surrounded by 2 coils of dimension $210 \mathrm{~mm}$ long, $263 \mathrm{~mm}$ inner bore radius. These are known as the absorber focus coils. They are superconducting and will be kept cold by a cryostat. These coils will reduce the beta function in the absorber and will aid cooling. They also provide magnetic capture of the beam and facilitate transport through the absorber windows. All three focusing sets will be powered from a single 300A, 10V supply.

The coupling coils will be placed at the centre of each set of 4 RF cavities. A single superconducting coil with an inner bore radius of $725 \mathrm{~mm}$ and length $250 \mathrm{~mm}$ will enable capture of the beam in each of the two RF sets. Each coil pair will be powered using a 300A, 10V supply.

Each tracker solenoid module is composed of 5 superconducting coils housed in a single cryostat. The uniform field region previously discussed is the centre coil. This magnet measures $1260 \mathrm{~mm}$ long and has a $255 \mathrm{~mm}$ bore radius. The two end coils and two matching coils are $120 \mathrm{~mm}$ and $202 \mathrm{~mm}$ long respectively and share the same bore dimensions as the centre coil.

The actual fields for all the magnets in this section are dependent on the operating conditions of MICE. They depend on the momentum and emittance of the beam being studied and as such will not be detailed here. Full discussion of the optics for the final 3 stages of MICE can be seen in $[82]$. 


\subsection{Conclusion}

The MICE experiment aims to show for the first time the concept of ionisation cooling. Using a number of muon beams differing in momentum and emittance one wishes to show a $10 \%$ cooling effect to a precision of $1 \%$ of this value. Furthermore, MICE should be a fully engineered cell of a Neutrino Factory cooling channel such that the required cooling can be achieved through subsequent cell placements. The supply of muons to the experiment is provided by a pion-muon decay beamline which transports a secondary beam created by a titanium target being inserted into the ISIS $800 \mathrm{MeV}$ proton synchrotron. Particle identification in MICE is achieved by a system including time-of-flight, cherenkov, calorimeter and tracking detectors with emittance measurement before entering and after exiting the experiment carried out by the latter. The success of MICE will be a major step towards realising a Neutrino Factory complex. 


\section{Chapter 6}

\section{Studies of particle production from the prototype MICE target}

\subsection{Introduction}

On the 1st/2nd November 2006 an experiment was carried out to study particle flux from the newly installed prototype MICE target. The experiment included two separate detectors, each consisting of a pair of scintillating hodoscope detectors, with one pair shielded by $5 \mathrm{~cm}$ of polyethylene and the other left unshielded. Both detectors were placed in the ISIS synchrotron vault at a production angle of $25^{\circ}$ to the newly installed target i.e. along the line-of-sight from the target to the hole in the ISIS vault which will transport particles into the MICE hall. The double hodoscope design enables coincidences to be established between tubes to eliminate backgrounds. The motivation for this test is to better establish the interaction between the MICE target and the ISIS beam and the implications this has for particle production rates into the MICE beamline, which is positioned at $25^{\circ}$ to ISIS. Additionally the test was the first demonstration of a MICE target operating in situ. This has far reaching effects for the MICE beamline design and the design and running of the MICE experiment. 


\section{$6.2 \quad$ ISIS}

The ISIS accelerator facility is housed at the Rutherford Appleton Laboratory in Oxfordshire, UK. It is a synchrotron primarily concerned with condensed matter research, which produces intense proton, neutron and muon beams. Initially $\mathrm{H}^{-}$ions are accelerated by a Radio Frequency Quadrupole (RFQ) accelerator operating at 202.5 MHz. The ions are then passed to the linac. This uses alternating current RF at a frequency of $202.5 \mathrm{MHz}$ to increase the energy of of the $H^{-}$to $70 \mathrm{MeV}$. The time between bunches at this point is $4.94 \mathrm{~ns}$. The ions are then stripped of electrons by passing a beam through a $0.3 \mu \mathrm{m}$ aluminium oxide stripping foil and injected into the synchrotron itself. Protons are injected into the ring at $70 \mathrm{MeV}$ kinetic energy. Injection lasts for 130 turns accumulating at maximum intensity of $2.5 \times 10^{13}$ protons per pulse. The machine cycle is $50 \mathrm{~Hz}$ accelerating to $800 \mathrm{MeV}$ over the $10 \mathrm{~ms}$ pulse duration. The synchrotron is comprised of ten dipole magnets to keep the beam travelling in a circular radius of $26 \mathrm{~m}$. Acceleration is carried out by six RF cavities which provide a peak accelerating voltage of $140 \mathrm{kV}$ per revolution.

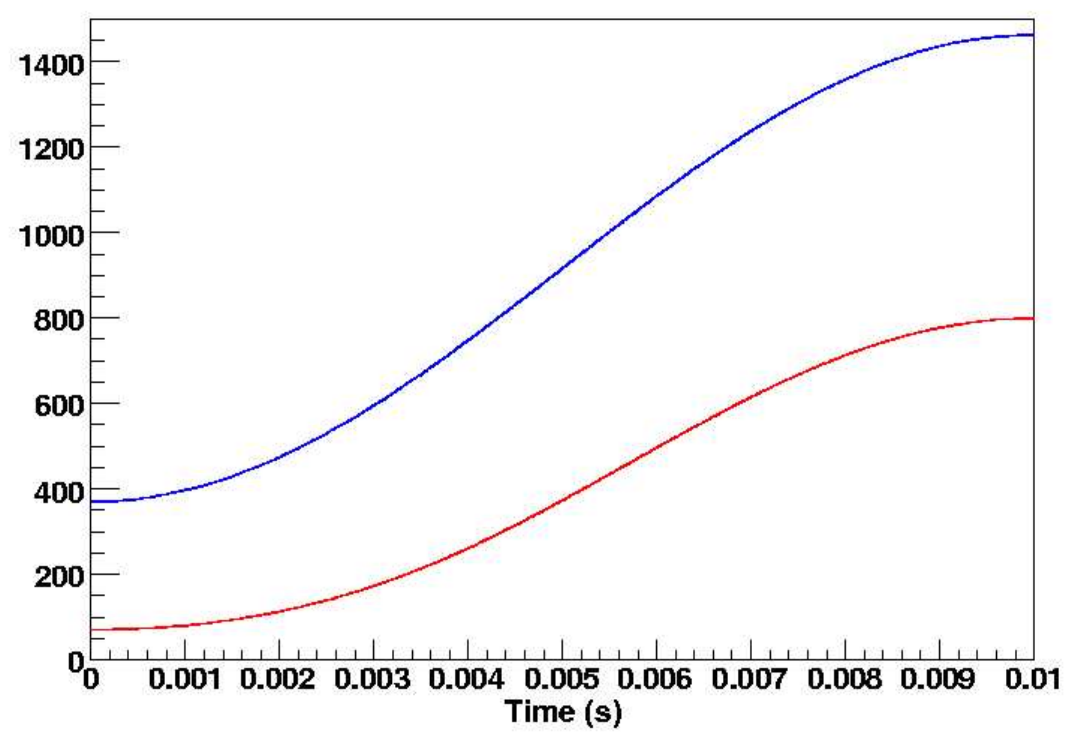

Figure 6.1: Evolution of energy (blue line, units are MeV) and momentum (red line, units are $\mathrm{MeV} / \mathrm{c}$ ) from injection to extraction

The evolution of the beam momentum and energy over the period from injection to extraction can be calculated from the ramping magnetic fields in the synchrotron over the cycle phase. The 
following equations describe the ramping of ISIS with the behaviour plotted for clarity in Figure 6.1:

$$
B=0.43687-0.26041 \times \sin (100 \pi(t+0.005))
$$

where B is the ramping magnetic field of the synchrotron dipoles in Tesla,

$$
\beta=\sqrt{\frac{\left(B \rho e / m_{0} c\right)^{2}}{1+\left(B \rho e / m_{0} c\right)^{2}}}
$$

is the proton velocity relative to the speed of light (c), $\rho=7 \mathrm{~m}$ is the dipole bending radius, $m_{0}$ is the proton rest mass and e is the electric charge,

$$
K . E=E_{0}(\gamma-1)
$$

is the kinetic energy of the protons, with $\gamma=\sqrt{\left(1-\beta^{2}\right)}$, the Lorentz relativistic factor,

$$
p c=E_{0} \sqrt{\gamma^{2}-1}
$$

is the momentum of the proton, and $E_{0}=m_{0} c^{2}$ is measured in $\mathrm{MeV}$.

For the target test, ISIS provided the following signals:

- Gated machine start (GMS);

- Total beam-loss;

- Beam-loss from SP7;

- ISIS current.

The GMS signal provides a trigger for target insertion. The ISIS current signal is extracted from the RMIm5 beam intensity toroid. Located around the inside circumference of ISIS are 39, 3m long coaxial ionisation chambers. These detectors are essential for beam diagnostics which in turn limit the ISIS intensity of $240 \mathrm{~kW}$. The beam-loss monitors detect stray protons via isotropically emitted evaporative neutrons produced when the escaped proton hits a machine component. The SP7 signal is extracted from the beam-loss monitor in ISIS Superperiod 7 (see Figure 6.2). This is the closest downstream beam-loss monitor downstream of the MICE target. 


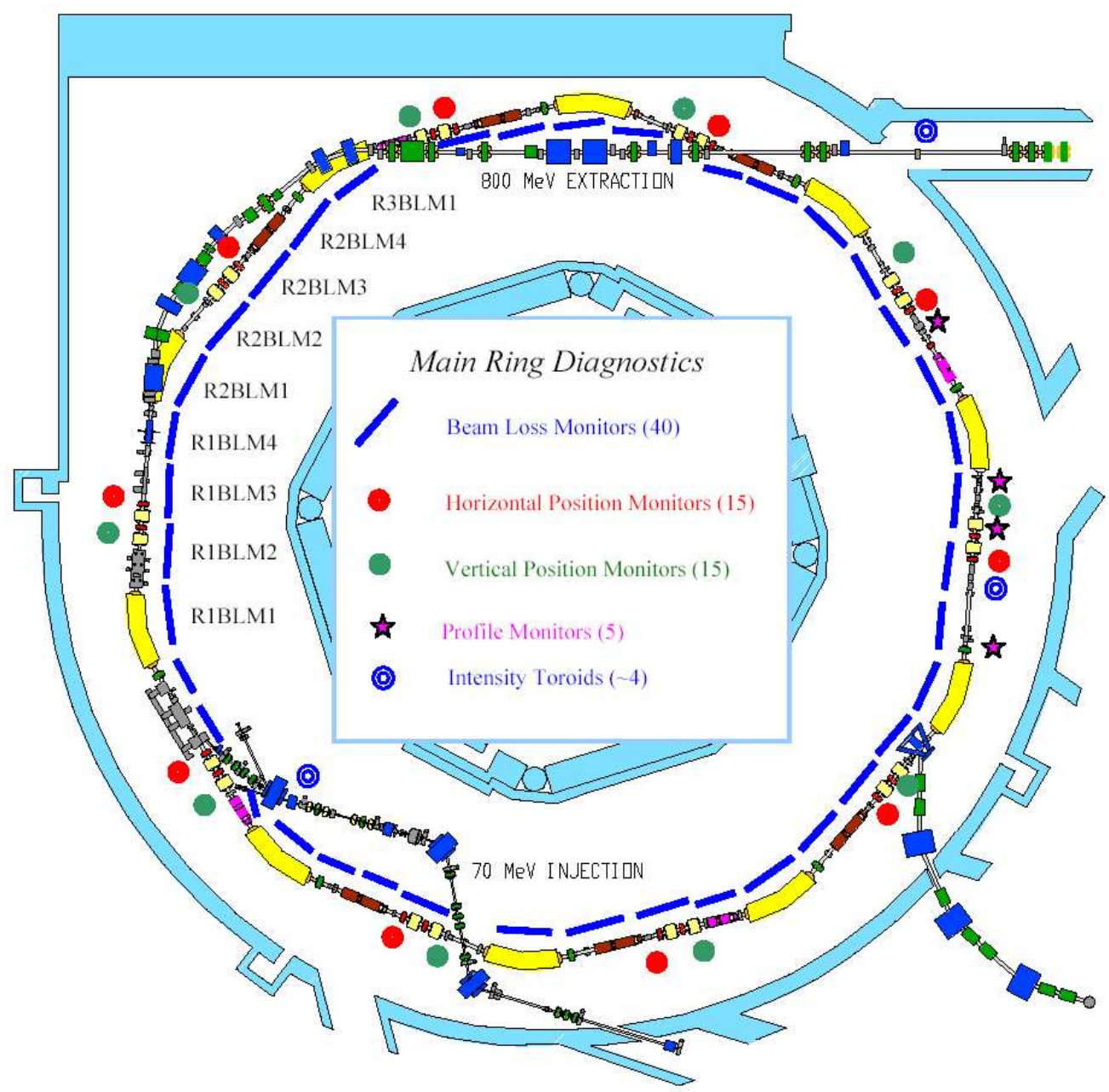

Figure 6.2: The ISIS neutron spallation source. Accelerator diagnostics are indicated. One should note the position of the old HEP beamline in the lower right of the diagram which is essentially the position of the MICE beamline. Superperiod 7 is the straight section immediately downstream of this [83].

\subsection{MICE target}

In preparation for the MICE experiment a new target system is being developed for installation onto the ISIS ring by teams from Sheffield University and RAL. The target is a $\left(10 \times 10 \times 1 \mathrm{~mm}^{3}\right)$ titanium fin which will be pulsed into the edge of the ISIS beam. Target insertion will pulse into the ISIS beam between 8-10 ms after the start of the machine cycle (i.e. the last $2 \mathrm{~ms}$ before extraction) and must be out of the beam during all other times. The final target design should be capable of pulsing, on demand, at rates of $1 \rightarrow 50 \mathrm{~Hz}$ with good reliability as it will have to operate for up to $10^{7}$ repetitions/year with very limited ability for access.

The target is a $\left(10 \times 10 \times 1 \mathrm{~mm}^{3}\right)$ titanium fin which will be pulsed into the halo of the MICE 
beam under the control of linear motion with radial magnets [84]. The baseline linear drive comprises of 24 coils driven by a commutator which supplies the correct currents. The shaft is kept on-axis via self-lubricating leaded bronze bearings. A schematic of the MICE target is shown in Figure 6.3. Position feedback is provided via an optical laser readout where a graduated scale is attached to the moving shaft giving positional precision of better than $0.2 \mathrm{~mm}$.

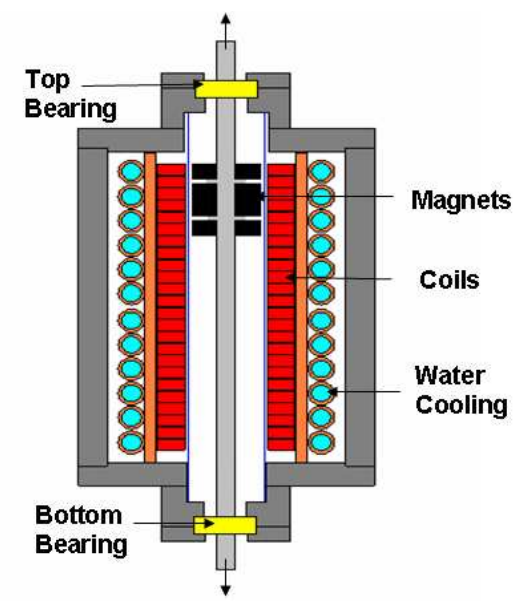

Figure 6.3: Schematic representation of the MICE target drive assembly

The MICE experiment will run parasitically to the normal operation of ISIS. The number of protons that the experiment will be able to intercept will be determined by the depth of insertion of the target into the beam and will be limited by the beam-loss monitors to limit the radiation dose in the vicinity of the ISIS synchrotron. This provides a hard constraint for target operation and consequently the MICE run scheme.

\subsection{Target test setup}

The target test set-up is centred around two regions, the ISIS vault and the MICE hall. A schematic representation is shown in Figure 6.4. The detectors themselves are positioned on the outside of the ISIS ring inside the vault. The shielded detectors were supported on a unistruct frame at a height of $115.5 \mathrm{~cm}$ with the unshielded detectors placed directly beneath. The ISIS beam-pipe is raised to a height of $140 \mathrm{~cm}$ such that the line of sight distance to the shielded and unshielded detectors are $990 \mathrm{~cm}$ and $980.3 \mathrm{~cm}$ respectively.

The power supplies and data-acquisition system (DAQ) for both pairs of detectors are con- 
tained in the MICE hall (hall 5.2). Power and signals are transfered via 50m BNC cables. The target test was controlled from hall 5.2 with much of the target control electronics also present.

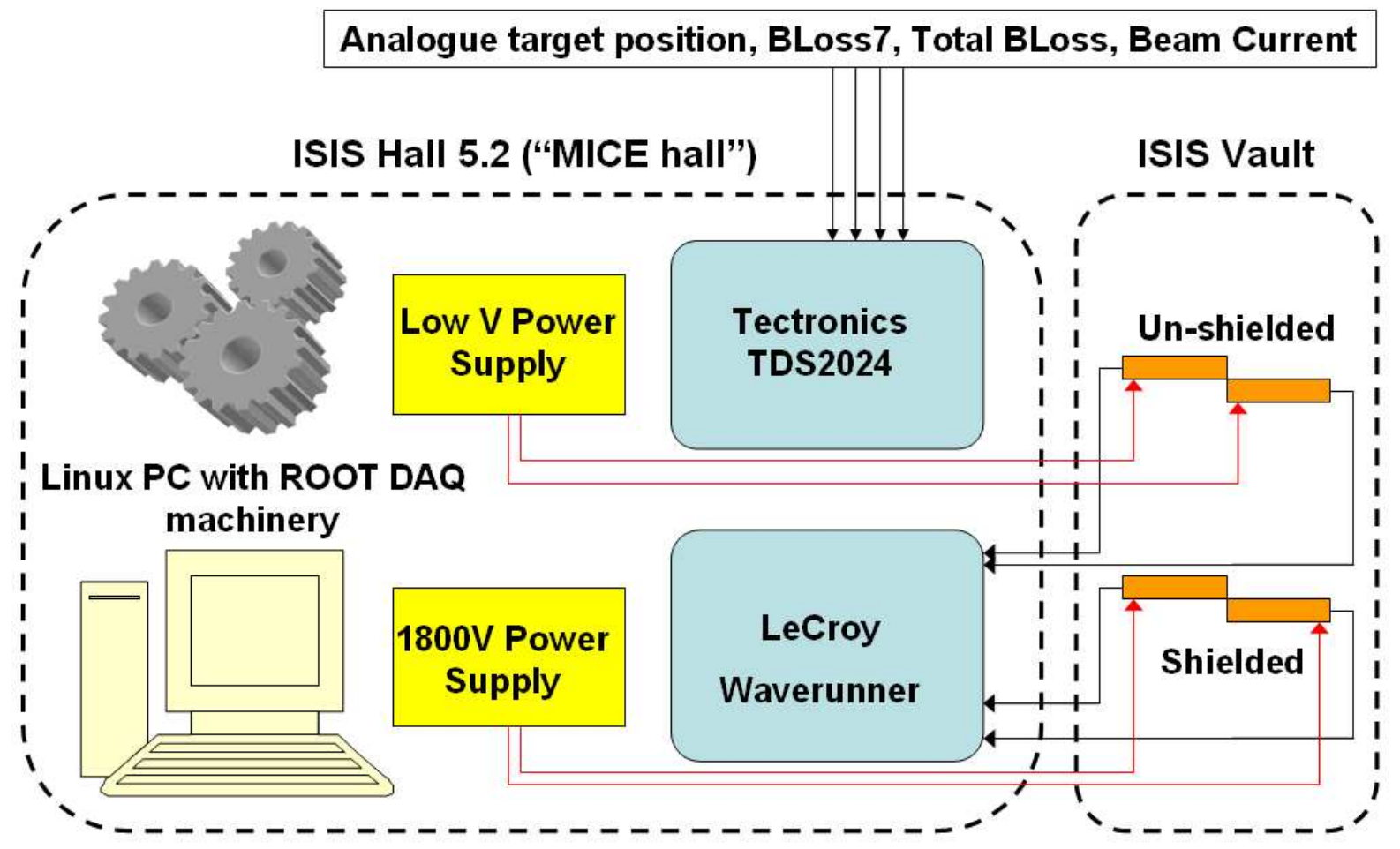

Figure 6.4: Target test setup

During this run the prototype target did not achieve (and was not expected to achieve) the final accelerations for the eventual MICE target for which the baseline is $\approx 1000 \mathrm{~ms}^{-2}(\approx 100 \mathrm{~g})$. The electronics used to drive the target were current-limited to $10 \mathrm{~A}$ which corresponded to a maximum acceleration of $157 \mathrm{~ms}^{-2}$. This restriction determined the ISIS cycle rate that was chosen i.e. base rate of $50 \mathrm{~Hz} / 64$. This corresponds to one pulse every $1.28 \mathrm{~s}$.

The run plan involved two separate run conditions. Initially, the target was configured such that it was lowered and held in the beam-pipe at different depths. The second run stage involved pulsing the target into the beam-pipe. Different levels of delay could be applied to the target, altering when the target dips into the beam and hence the amount of beam intercepted. 


\subsection{Unshielded counters}

The purpose of the unshielded detectors is to measure the absolute particle rate at a production angle which can be used to estimate the total flux of particles into the MICE acceptance. The detector is comprised of a dual phototube configuration with each tube observing light produced from one of two small scintillator blocks measuring $1 \mathrm{~cm}^{3}$ each.
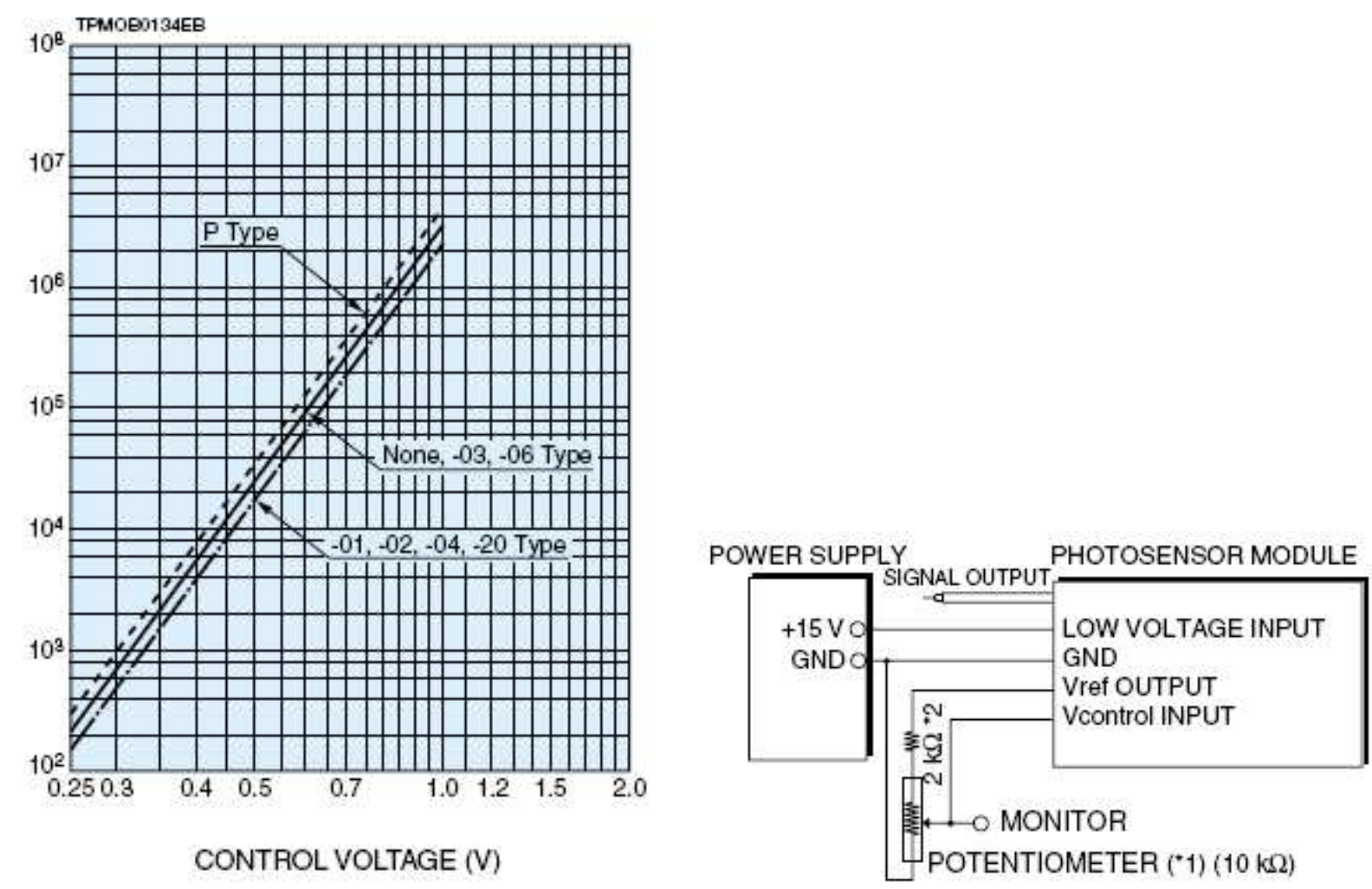

Figure 6.5: High gain dependency of the unshielded detector phototubes as a function of the control voltage applied (left panel). The electrical layout of the phototubes (right panel)

To reduce possible pile up effects due to the high rate, the photomultiplier tubes chosen were Hamamatsu H5783(P). These tubes have small physical dimensions and are extremely fast. The rise-time for the tubes is $0.78 \mathrm{~ns}$ with a short transit time. The gain of the H5783(P) tubes can be altered across a large range of $7.5 \times 10^{5}-1 \times 10^{6}$ via a potentiometer (see Figure 6.5 ). The tubes operate with an encapsulated base driven by a low voltage of $V_{\max }=15 \mathrm{~V}$. The surfaces are polished and wrapped in aluminium foil to improve light yield. Optical coupling to the PMT is mediated by optical grease and the entire PMT-scintillator coupling region is encased in black tape to provide a complete light-tight seal. To further improve the light seal and to provide 


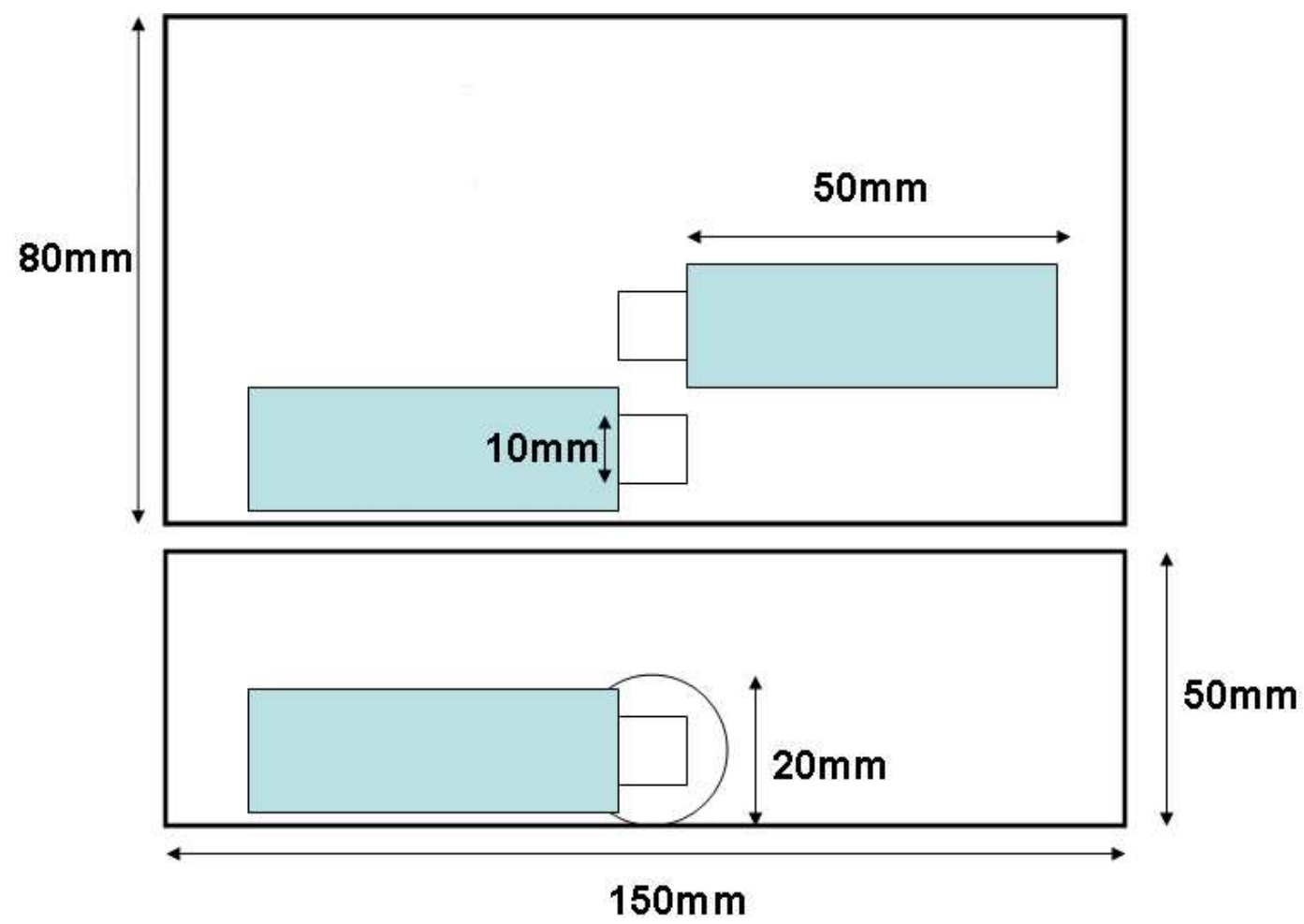

Figure 6.6: Representation of the unshielded detector showing PMT layout and housing.

rigidity to the detector both hodoscopes were mounted in a black plastic box with two LEMO connector signal outputs and two LEMO connector inputs for power. A schematic diagram of this set-up is shown in Figure 6.6. Power is supplied to each PMT from a low-voltage digital supply located in the MICE hall via a 50m BNC cable and BNC-lemo connector. The signal is transferred from the ISIS vault to the MICE hall via a LEMO-BNC connector, then 50m BNC cables to the LeCroy oscilloscope.

\subsection{Shielded counters}

The shielded detectors are recycled from the MUSCAT experiment [85]. Again this is comprised of two hodoscope detectors. The PMT's in this case are larger H6533 1" diameter phototubes. These tubes have a rise time of $0.7 \mathrm{~ns}$ and transit time of $10 \mathrm{~ns}$. The phototubes are shielded with $5 \mathrm{~cm}$ of polyethylene through almost $360^{\circ}$. Unshielded regions are located at the base end of each phototube to allow cable access. The shielded region along the beam line-of-sight is $5 \mathrm{~cm}$. The light to each tube is provided by a $3 \mathrm{~mm} \times 3 \mathrm{~mm} \times 30 \mathrm{~mm}$ pencil scintillator. High voltage 
power was supplied to both these tubes via 50m BNC cables from two $1800 \mathrm{~V}$ power supplies housed in Hall 5.2. As was the case with the unshielded detectors the signals from these tubes were transfered to the LeCroy oscilloscope.

\subsection{Data acquisition}

The data-acquisition for the test is completely carried out by digital oscilloscopes. This choice was made because of the availability of the scopes and ease of implementation. The scope buffers were interfaced to a Linux PC via GPIB. The acquisition software was written using ROOT. For each run a ROOT histogram is produced.

The data acquisition requirements are different for the detectors housed within the ISIS vault and the target and ISIS signals. The "slow" signals i.e. the non detector signals did not require very fine grained time resolution. What was more important was to sample over a longer time period such that one could observe the whole ISIS pulse. These signals were read-out by a TektronixTDS2024 digital scope [86]. The scope memory depth is 2.5 samples per channel with a recording period from -0.005 to $0.02 \mathrm{~s}$. This acquisition setup allows for a timing resolution of $10 \mu s$ for the target analogue signal, total beamloss, beamloss in straight 7 and the ISIS current. The signals from all four phototubes require much finer resolution than this to resolve individual pulses. Hence the detector signals are read out by a LeCroy Waverunner 6100 [87]. This scope has a $1 \mathrm{Mpt}$ long buffer per channel. The recording period for this acquisition is $10 \mathrm{~ms}$ from 0 to $0.01 \mathrm{~s}$. Under these conditions the timing resolution is $10 \mathrm{~ns}$. The buffers are read-out to a desktop PC running Linux via a GPIB interface. The data is saved in the form or Root histograms which were then copied to machines at Sheffield and Glasgow Universities for further analysis.

\subsection{Simulations}

The simulations of the shielded and unshielded detectors were modelled using a combination of GEANT4 (using the LHEP Binary Cascade hadronic engine) and MARS. Both sets of detectors were placed at a $25^{\circ}$ angle from the ISIS accelerator at the correct line of sight distance from the target (shown in Table 6.2). All relevant materials were taken into consideration. Using a 


\begin{tabular}{|c|c|c|}
\hline Parameter & ISIS/target signals & Detector signals \\
\hline \hline Scope & Tektronix TDS2024 & LeCroy Waverunner 6100 \\
Number of channels & 4 & 4 \\
Buffer depth $(\mathrm{kS})$ & 2.5 & 1000 \\
Sample rate $(\mathrm{GS} / \mathrm{s})$ & 2 & 5 \\
Bandwidth & $200 \mathrm{MHz}$ & $1 \mathrm{GHz}$ \\
Sampling range & $-5 \rightarrow 0.02 \mathrm{~s}$ & $0 \rightarrow 0.01 \mathrm{~s}$ \\
Time resolution & $10 \mu \mathrm{s}$ & $10 \mathrm{~ns}$ \\
\hline
\end{tabular}

Table 6.1: Data acquisition summary

simulation of the MICE target undertaken previously all particles which fell into the angular acceptance of the MICE channel were sampled i.e. $x_{\max }^{\prime}=40 \operatorname{mrad}, y_{\max }^{\prime}=20 \mathrm{mrad}$. The target simulations were carried out using GEANT4 and MARS codes in which 10 million, 1400 $\mathrm{MeV} / \mathrm{c}$ protons were incident on a titanium target of the appropriate MICE dimensions. Thus, this simulation corresponds to a proton energy about 9ms after ISIS injection.

\begin{tabular}{c|c|c} 
Parameter & Unshielded detectors & Shielded detectors \\
\hline \hline Area of target $\left(\mathrm{mm}^{2}\right)$ & 100 & 100 \\
Distance to target $(\mathrm{cm})$ & 990 & 980.3 \\
Target sample simulation & MARS/GEANT4 & MARS/GEANT4 \\
Shielding & None & $5 \mathrm{~cm}$ of polyethylene
\end{tabular}

Table 6.2: Simulation parameters

The particles from the target simulation are then tracked using a GEANT4 simulation within the MICE acceptance, which forms an annulus, with central axis (z axis) placed at a $25^{\circ}$ angle. This sampled beam, serving as an input, allows tracking of the simulated target beam through the air region to the detectors and in the case of the shielded detector also through $5 \mathrm{~cm}$ of shielding. The detectors were initially modelled using the exact transverse dimensions of the scintillators. The singles statistics recorded on the upstream face of the scintillators were too low and the target data-set was of a fixed size. To improve this the transverse detector area was increased to $40 \times 40 \mathrm{~cm}^{2}$. The transverse particle density was checked to be flat as a function of area over this range and hence this approximation was valid (Figure ??). The results for yields of individual particle species incident on the upstream face of both detectors tracked using the MARS and GEANT4 distributions can be seen in Tables 6.3 and 6.4 respectively. The corresponding momentum distributions for this data at the detector locations can be seen in 
Figures 6.8, 6.9, 6.10, 6.11.

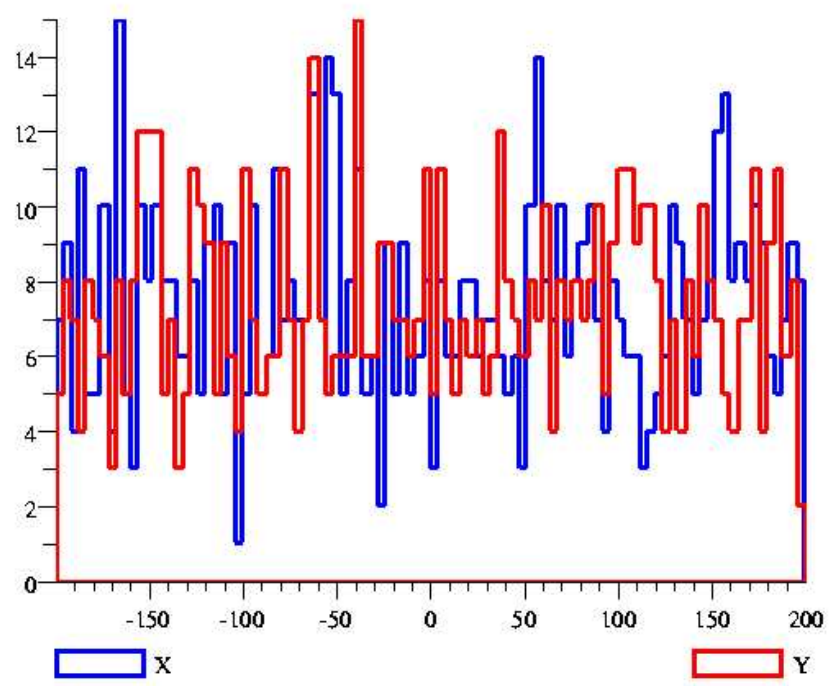

Figure 6.7: Transverse particle density detected in the $40 \times 40 \mathrm{~cm}^{2}$ detector using the MARS target distribution, showing that it is essentially flat in the $40 \times 40 \mathrm{~cm}^{2}$.

\begin{tabular}{c|c|c|c} 
Particle species & Unshielded detector & Shielded detector & $\%$ difference \\
\hline \hline Protons & 261 & 226 & -13.4 \\
Neutrons & 446 & 405 & -9.2 \\
$\pi^{+}$ & 6 & 7 & +16.7 \\
$\pi^{-}$ & 2 & 2 & 0 \\
$\mu^{+}$ & 4 & 4 & 0 \\
$\mu^{-}$ & 1 & 1 & 0 \\
$e^{-}$ & 9 & 10 & 10 \\
Total singles & 729 & 655 & -10.2
\end{tabular}

Table 6.3: Un-normalised rates for both shielded and unshielded detectors $\left(40 \times 40 \mathrm{~cm}^{2}\right)$ using MARS derived target yields. Also shown is the difference between results as a percentage of the unshielded rate for $1 \times 10^{7}$ protons incident on target. Statistics are very low for meson and leptons and hence the $\%$ difference yields no real result, they are included for completeness

\subsubsection{Triggering efficiency}

The probability that the detectors will record a hit from passage of a charged particle is given by the coincidence of the two detectors. We obtain the triggering efficiency from the simulation of both detectors. A simple definition of the efficiency for obtaining a coincident signal in both is by looking at the number of particles which hit the front face of the first scintillator block 


\begin{tabular}{c|c|c|c} 
Particle species & Unshielded detector & Shielded detector & $\%$ difference \\
\hline \hline Protons & 303 & 217 & -28.4 \\
Neutrons & 397 & 391 & -1.5 \\
$\pi^{+}$ & 20 & 20 & 0 \\
$\pi^{-}$ & 13 & 13 & 0 \\
$\mu^{+}$ & 17 & 16 & -5.9 \\
$\mu^{-}$ & 9 & 9 & 0 \\
$e^{-}$ & 2 & 1 & -50 \\
$e^{+}$ & 21 & 7 & -66 \\
Total singles & 861 & 674 & -21.7
\end{tabular}

Table 6.4: Un-normalised rates for both shielded and unshielded detectors $\left(40 \times 40 \mathrm{~cm}^{2}\right)$ using GEANT4 derived target yields for $1 \times 10^{7}$ protons incident on target. Also shown is the difference between results as a percentage of the unshielded rate. Statistics are very low for meson and leptons and hence the $\%$ difference yields no real result, they are included for completeness

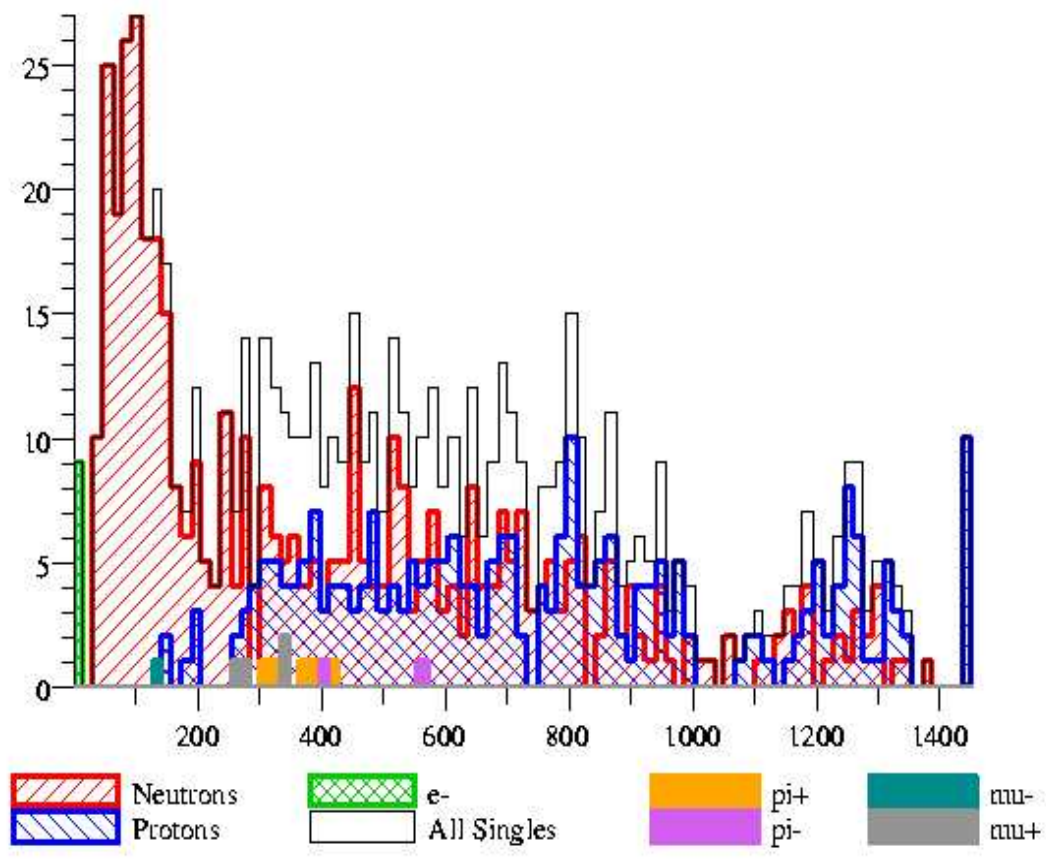

Figure 6.8: Simulated momentum truth distribution recorded by the unshielded detectors $(40 \times$ $40 \mathrm{~cm}^{2}$ ) for all incident particles tracked from MARS target yields, for $1 \times 10^{7}$ protons incident on target

compared with those incident on the second scintillator block (see Table 6.5). This is equivalent to ensuring that a particle that traverses the first detector will also record a hit in the second detector. This technique can be used to add a normalisation factor when comparing simulation to data. 


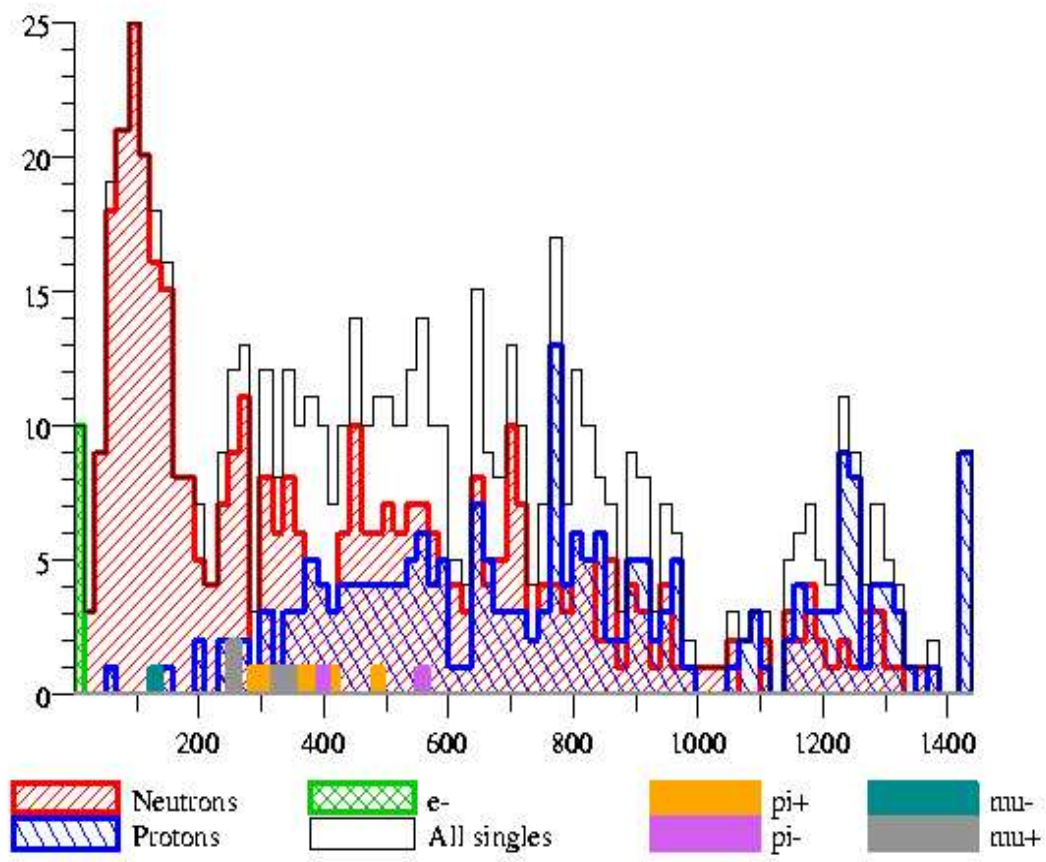

Figure 6.9: Simulated momentum truth distribution recorded by the shielded detectors $(40 \times$ $40 \mathrm{~cm}^{2}$ ) for all incident particles tracked from MARS target yields, for $1 \times 10^{7}$ protons incident on target

ISIS is a neutron spallation source and as such one has a large yield of neutrons. The neutrons produced are fast and therefore difficult to detect in a thin scintillator. Neutron elastic scattering cross-sections for non-thermal neutrons, as produced by the target, are orders of magnitudes lower than thermal cross-sections [88]. For a neutron to be detected in our experiment it must produce a recoil proton which deposits enough energy to give a signal in the first scintillator block and the second. To provide an upper limit on our detection efficiency for neutrons we use data and parameterisations given in [88]. If $\mathrm{N}$ is the number density of the target nuclei, $\sigma_{s}$ is the scattering cross-section for these nuclei and $d$ is the path length through the detector for incident neutrons then the counting efficiency is given by (6.5):

$$
\epsilon=\frac{N_{H} \sigma_{H}}{N_{H} \sigma_{H}+N_{C} \sigma_{C}}\left[1-\exp \left[-\left(N_{H} \sigma_{H}+N_{C} \sigma_{C}\right) d\right]\right]
$$

where the $\mathrm{H}$ and $\mathrm{C}$ denote the values for hydrogen and carbon and the cross-sections are given by (6.6): 


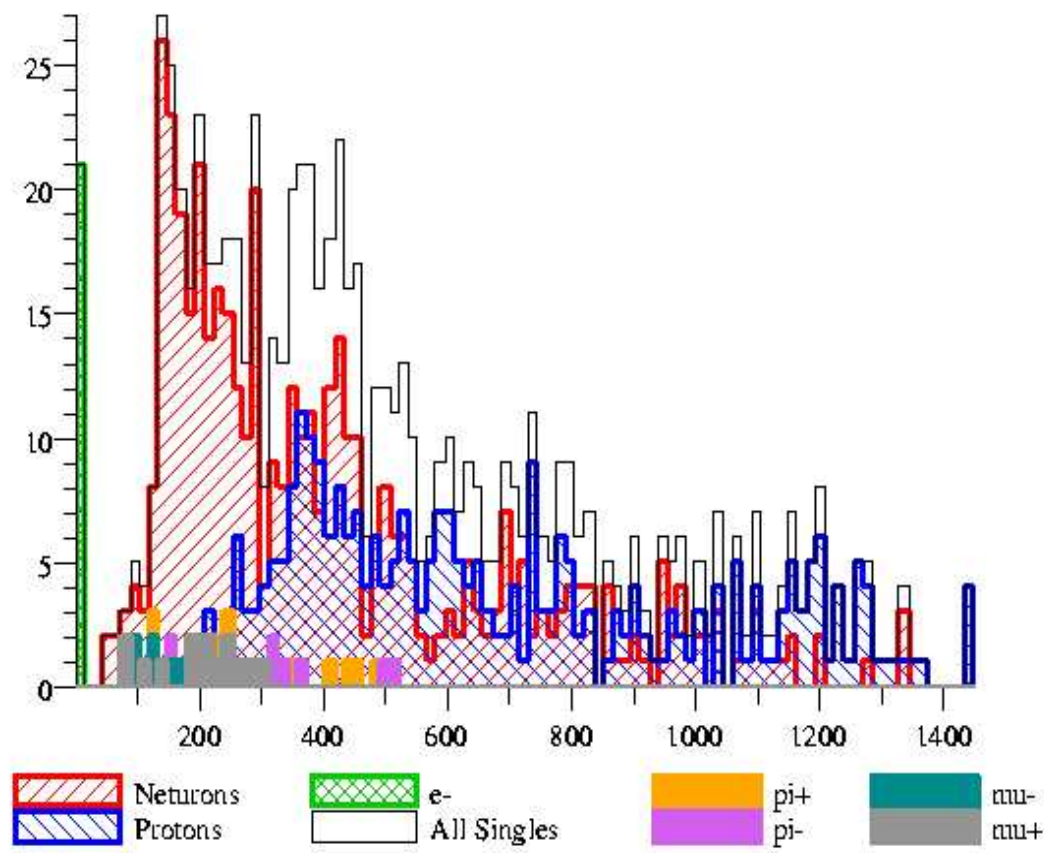

Figure 6.10: Simulated momentum truth distribution recorded by the unshielded detectors $\left(40 \times 40 \mathrm{~cm}^{2}\right)$ for all incident particles tracked from GEANT4 target yields, for $1 \times 10^{7}$ protons incident on target

$$
\sigma_{H}\left(E_{n}\right)=\frac{4.83}{\sqrt{E_{n}}}-0.578 \text { barns }, \sigma_{C}\left(E_{n}\right)=\frac{2.71}{\sqrt{E_{n}}}-0.210 \text { barns }
$$

The hydrogen parametrisation is given in [88] and the carbon parametrisation is extracted from data also shown in [88]. In addition to this efficiency we estimate the efficiency of the recoil proton giving a signal in the second scintillator as the efficiency as calculated previously for the protons. The efficiency curve from the parametrised equations were used to calculated the efficiency of detection in $1 \mathrm{MeV} / \mathrm{c}$ bins across the relevant range (see Figure 6.12). Inspection of the neutron production kinetic energy spectra from both GEANT4 and MARS simulations binned in $1 \mathrm{MeV}$ increments allow for an estimation of the total number of neutrons from the simulations that are expected to be detected and the average efficiency of detection. For the shielded and unshielded detectors using the MARS target distributions one expects to detect 14.1 and 16.0 neutrons respectively. For the GEANT4 target distribution one expects for the shielded and unshielded detectors 4.8 and 4.5. The source of the difference being accounted for 


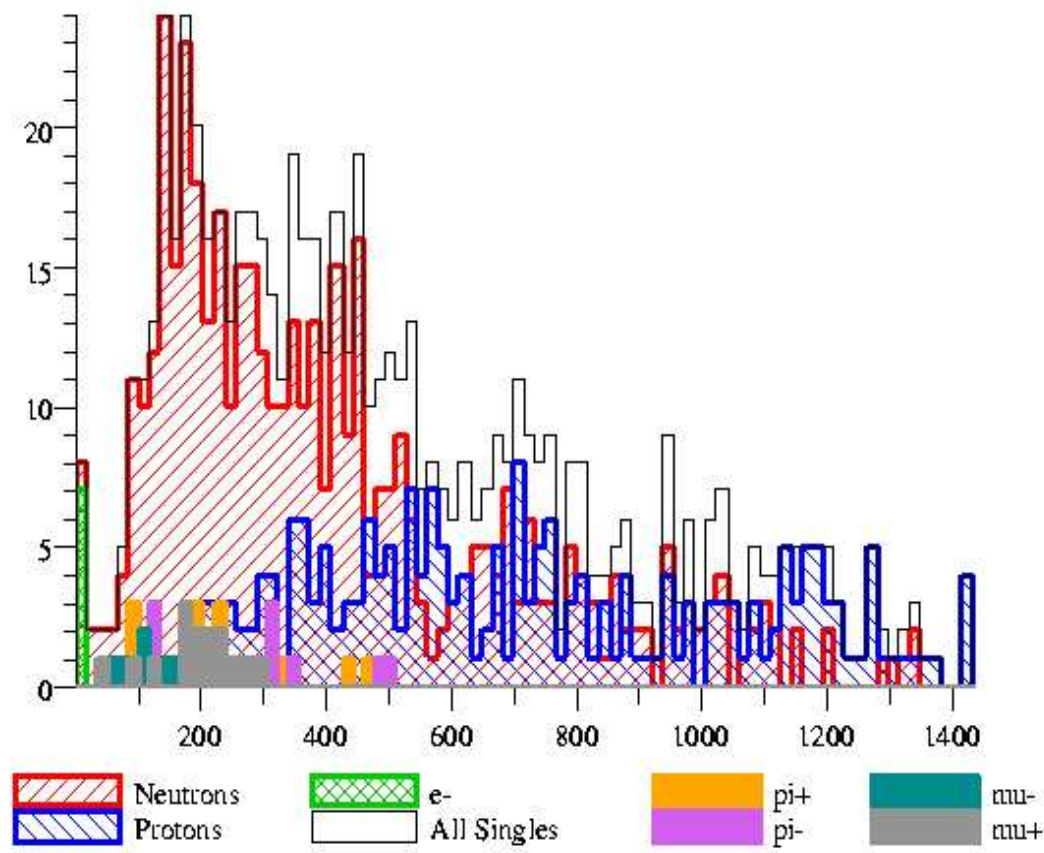

Figure 6.11: Simulated momentum truth distribution recorded by shielded detectors $(40 \times$ $40 \mathrm{~cm}^{2}$ ) for all incident particles tracked from GEANT4 target yields, for $1 \times 10^{7}$ protons incident on target

by MARS producing neutrons with slightly lower kinetic energies.

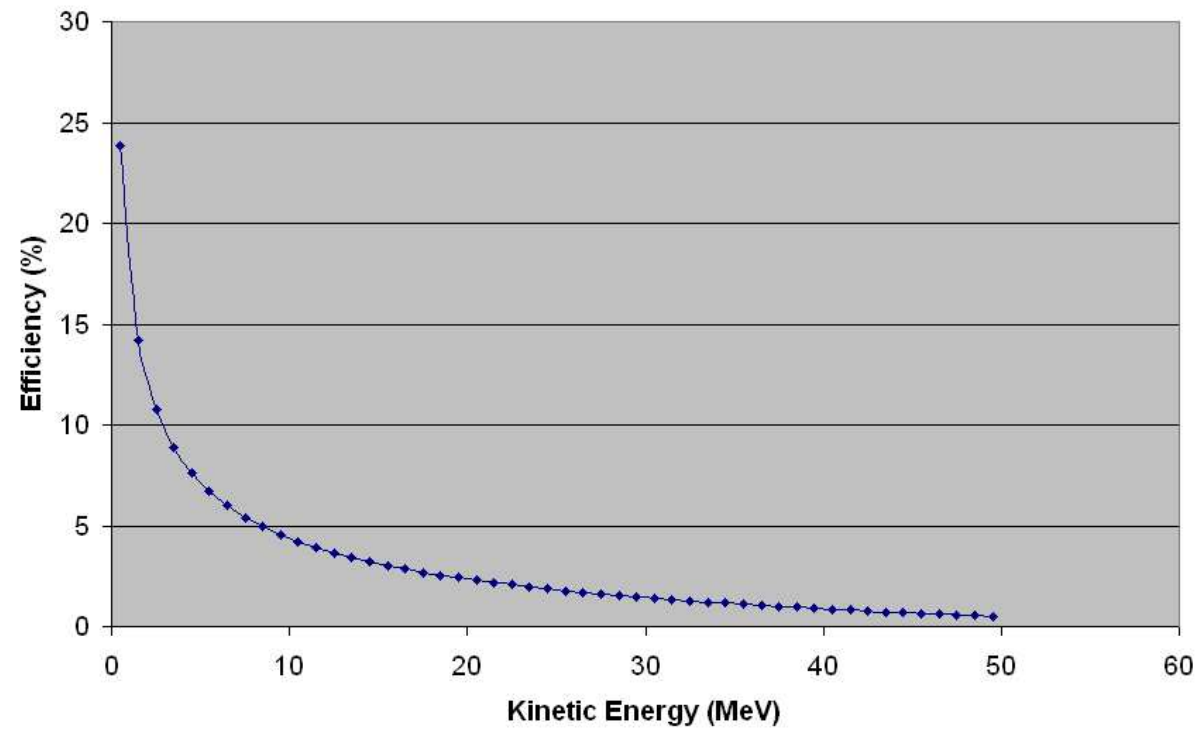

Figure 6.12: Parametrised neutron efficiency curve as a function of incident neutron energy 


\begin{tabular}{|c|c|c|}
\hline Particle species & Efficiency (un-shielded)(\%) & Efficiency (shielded) $(\%)$ \\
\hline \hline Protons & 96.2 & 97.5 \\
Neutrons & 5.9 & 5.7 \\
$\pi^{+}$ & 100 & 85.7 \\
$\pi^{-}$ & 100 & 100 \\
$\mu^{+}$ & 100 & 100 \\
$\mu^{-}$ & 100 & 100 \\
$e^{-}$ & 100 & 80 \\
\hline Total singles & 97.7 & 96.8 \\
\hline
\end{tabular}

Table 6.5: Un-normalised rates at upstream face of both scintillators for shielded and unshielded detectors from the MARS target yield. This gives an estimate of the efficiency of detecting a coincidence in both phototubes

\begin{tabular}{|c|c|c|}
\hline Particle species & Efficiency (un-shielded)(\%) & Efficiency (shielded) $(\%)$ \\
\hline \hline Protons & 85.1 & 91.2 \\
Neutrons & 2.2 & 2.5 \\
$\pi^{+}$ & 95.0 & 90 \\
$\pi^{-}$ & 92.3 & 92 \\
$\mu^{+}$ & 94.1 & 93.8 \\
$\mu^{-}$ & 100 & 100 \\
$e^{-}$ & 100 & 100 \\
\hline Total singles & 92.5 & 94.5 \\
\hline
\end{tabular}

Table 6.6: Un-normalised rates at upstream face of both scintillators for shielded and unshielded detectors from the GEANT4 target yield. This gives an estimate of the efficiency of detecting a coincidence in both phototubes

\subsection{Detector Analysis}

\subsubsection{Event Selection}

The data collected by both shielded and unshielded detectors can be seen in Table 6.7. The runs labelled "Static" were runs where the target was inserted at a fixed position into the beam. The runs with a time label in seconds denote the delay between the start of the target of the target acceleration and the start of the ISIS spill. A longer delay (i.e. 0.0145s) signifies a greater insertion into the beam.

The data from each target pulse is recorded as a ROOT histograms. To perform analysis it was essential to convert these histograms to ntuples. This process was complicated by the different timing resolutions for both scopes. The decision was made to use the timing resolution 
of the LeCroy scope as the stepping size in the newly created ntuples. To do this the data from the coarser grained Tektronics scope was modified such that these ntuples could be created.

\begin{tabular}{|l|l|l|}
\hline Target motion & Number of files & File ID \\
\hline \hline Static $0 \mathrm{~mm}$ & 4 & $180544,200914,200954,201146$ \\
Static $12 \mathrm{~mm}$ & 3 & $200327,200423,200545$ \\
Static $13.5 \mathrm{~mm}$ & 3 & $195409,195629,195817$ \\
Static $15 \mathrm{~mm}$ & 3 & $192821,193209,193306$ \\
Static $16.5 \mathrm{~mm}$ & 3 & $193640,194049,194420$ \\
Static $18 \mathrm{~mm}$ & 1 & 194732 \\
$0.0045 \mathrm{~s}$ & 3 & $202239,202421,202501$ \\
$0.0082 \mathrm{~s}$ & 3 & $202853,202930,203011$ \\
$0.0125 \mathrm{~s}$ & 5 & $223951,224117,224212,224300,224339$ \\
$0.0130 \mathrm{~s}$ & 3 & $203400,203547,203641$ \\
$0.0135 \mathrm{~s}$ & 16 & $214335,214430,214610,214838,215028,215122$, \\
& & $222653,222920,222958,223106,223253,223332$, \\
$0.0140 \mathrm{~s}$ & 4 & $223416,223505,223552,223638$ \\
$0.0145 \mathrm{~s}$ & 5 & $221030,221135,221347,224900,225112$ \\
\hline
\end{tabular}

Table 6.7: Summary of data runs and target operating conditions

The next stage in the analysis was the identification, clustering and evaluation of pulses in the data. Before this could be achieved the voltage signals from each PMT were discriminated. The level of discrimination is unique to each of the four photomultiplier tubes and the level chosen was determined from the bin height distribution recorded from each tube. The run used to create these spectra was the final run for which the target delay was 0.0145s. This was chosen as it has relatively high statistics. A value of $3 \sigma$ from the noise level for each of the four distributions was chosen as the discrimination level. The bins below threshold were set to zero. The choice of discrimination level was checked by calculating the coincidence frequency at both $2 \sigma$ and $5 \sigma$, at $5 \sigma$ no reduction was seen compared to the $3 \sigma$ level with the equivalent comparison at $2 \sigma$ showing a reduction.

An algorithm based on the signal shape was used to identify a pulse. The algorithm identified a pulse by looking for bins that were above threshold with the previous bin at the zero level. All consecutive bins above threshold were counted as part of the same pulse until another bin was encountered which was below threshold. This constituted a pulse. In addition, the pulse length and the sum of the voltage of the pulse were calculated. These quantities were measured 
to check if it would be possible to distinguish between protons and minimum ionising particles (MIPS) such as pion and muons. Unfortunately the spectra produced did not show any features which could be used to identify these different populations.

The next stage was to identify coincidences between both PMTs in the unshielded detector and similarly with the shielded detectors. After clusterisation had taken place, the clusters identified in the data from both PMTs were compared and those clusters whose leading edges coincided with a tolerance of $\pm 10 \mathrm{~ns}$ were selected as true coincidences. An example of a coincidence observed in the raw data collected in both unshielded detectors can be seen in Figure 6.13. The sum of coincident hits in both detectors summed over all runs can be seen in Figures 6.15 and 6.16. For the runs in which the target was dipped into the beam (red histograms), the number of hits increases at the end of the $10 \mathrm{~ms}$ spill because, as the target inserts further into the beam, this causes production of a larger number of secondary particles from the high energy protons interacting in the target. As the beam accelerates it shrinks, so the target position needs to keep up with the edge of the beam to produce particles. Figure 6.14 shows the motion of the target during the $10 \mathrm{~ms}$ spill for the different target conditions. The peak between 5 and $6 \mathrm{~ms}$ corresponds to the situation in which the target accelerated faster than the beam could shrink, so there was a greater intersection of the target with beam. For the runs in which the target was at a fixed position (blue histogram) the only time in which the beam intersects the target is at the beginning of the spill, when the beam is largest. 


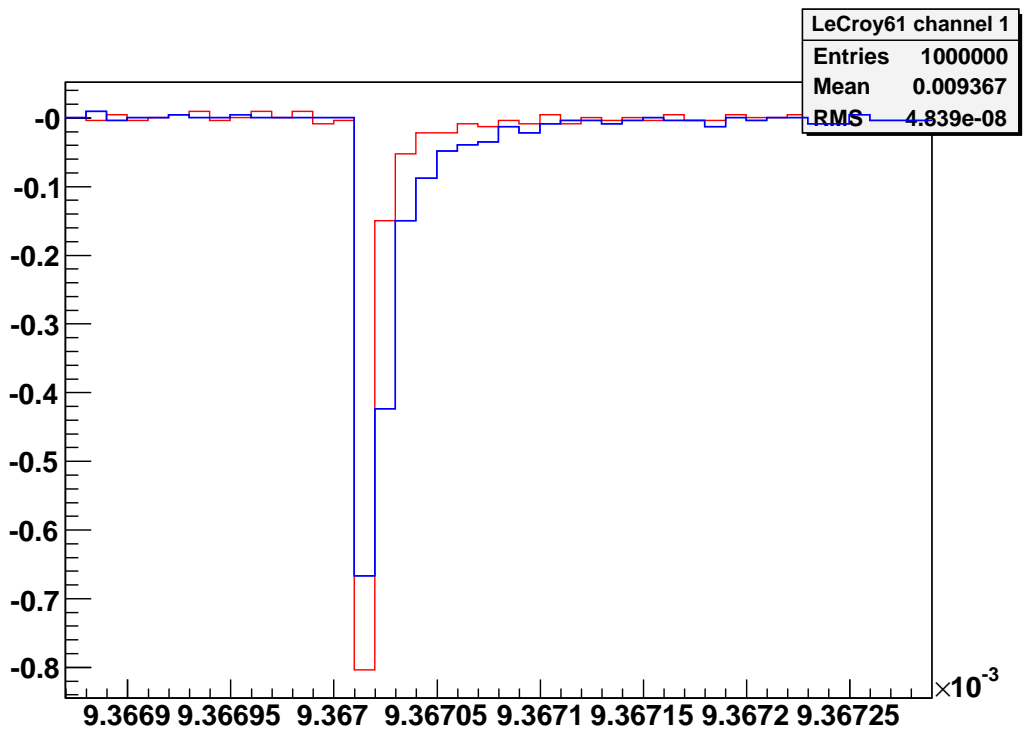

Figure 6.13: A Typical coincidence observed in raw data observed in both unshielded detectors. Each colour denotes the signal from an individual tube

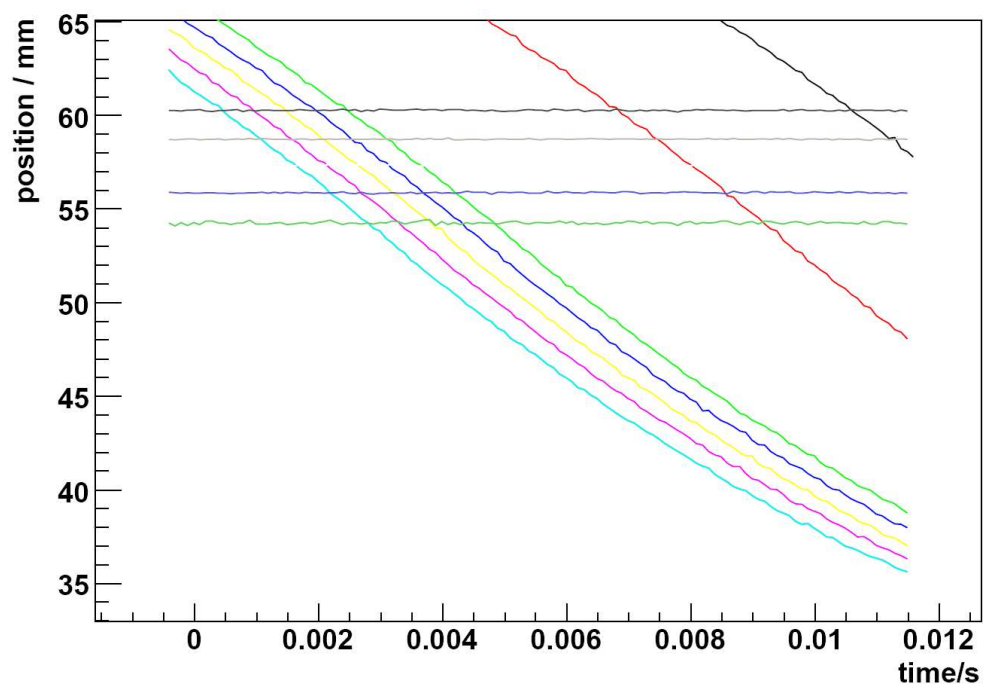

Figure 6.14: Evolution of the target position for static and pulsed run conditions over the 10ms spill [89] 


\section{Coincident hits in unshielded detectors}

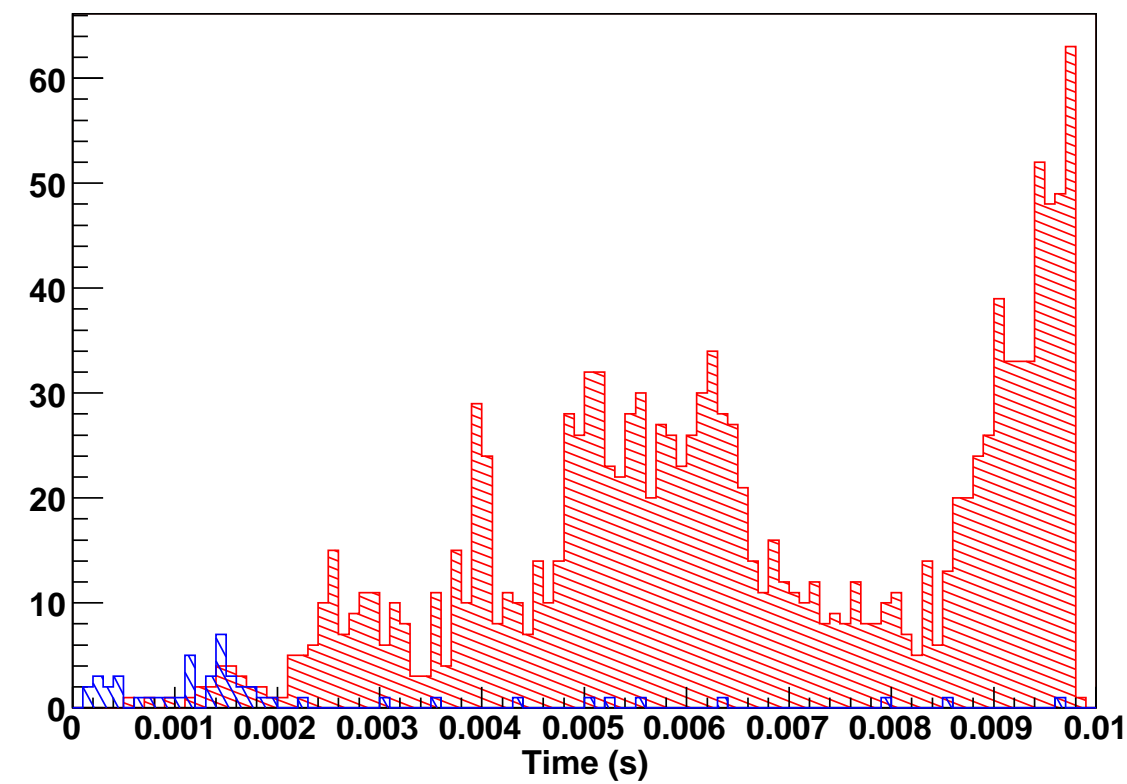

Figure 6.15: The complete data-set for all run conditions are plotted for the unshielded detector as a function of time during the pulse. The red histograms show coincident hits for runs when the target was dipped into the beam. The blue histograms are coincident hits for the runs where the target was held inside the beam.

\section{Coincident hits in shielded detectors}

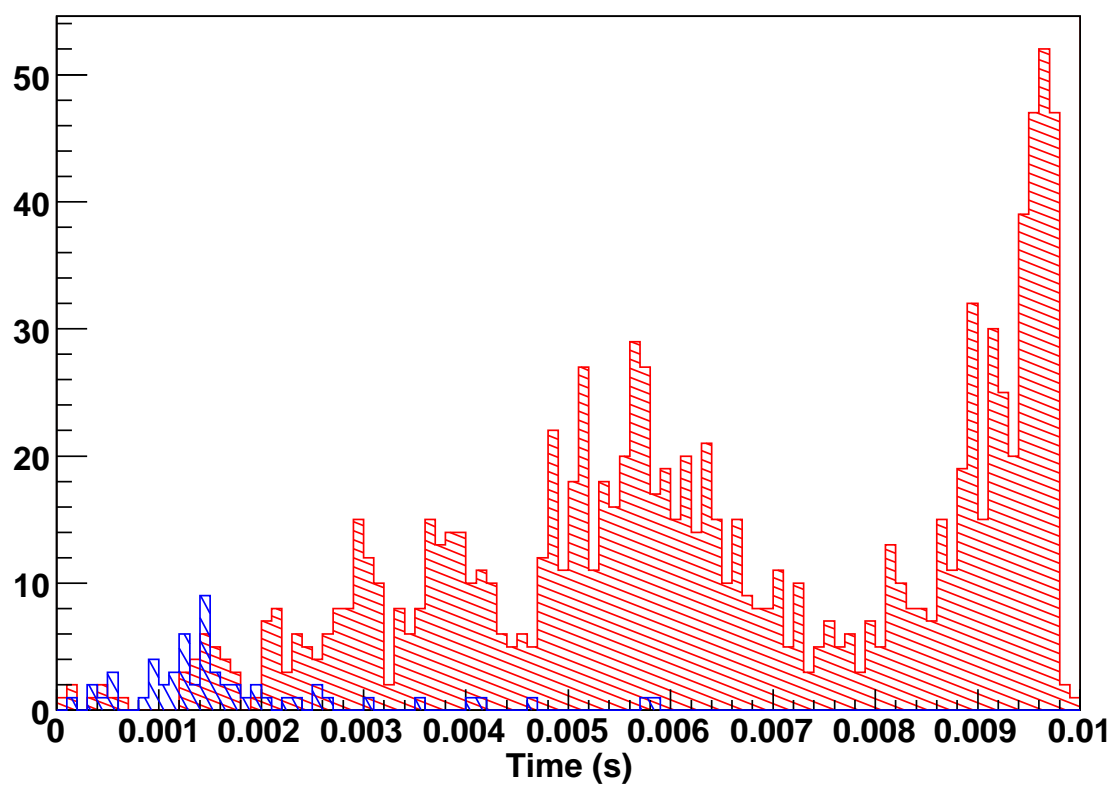

Figure 6.16: The complete data-set for all run conditions are plotted for the shielded detector as a function of time during the pulse. The red histograms show coincident hits for runs when the target was dipped into the beam. The blue histograms are coincident hits for the runs where the target was held inside the beam. 


\subsubsection{Results of investigation of SP7 measured beam loss as a function of particle yields}

It is important that the MICE experiment be able to monitor the beam loss from ISIS when running. As MICE will operate parasitically on ISIS, beam loss due to the MICE target could induce higher than acceptable radiation levels and could limit the running conditions for MICE. This will arguably be the biggest external risk to the experiment, as ISIS will evaluate the effect of the MICE induced beam loss and, if necessary, modify the running of MICE. This in turn will have an effect on run scheduling and hence could affect physics reach. The measurement of beam loss in ISIS is carried out by monitors around the inside circumference of the machine as previously discussed.

The key monitor that will be likely to impose constraints on MICE is the beam loss monitor in super-period 7 (SP7) i.e. the device monitoring the proton flux lost in the vicinity of the MICE target. Under normal ISIS running, the measured loss in SP7 is zero, hence any loss measured there during MICE running would be assumed to be from the MICE target itself. ISIS is tripped locally by individual signals from any of the ISIS monitors that exceed the trip limit for 20 pulses. To reflect the normally low loss in SP7 the level is set very low by ISIS at $50 \mathrm{mV}$. To compare, the signal limit for the monitors immediately upstream of injection (SP1) can be up to $3 \mathrm{~V}$. Although the $50 \mathrm{mV}$ level will be able to be negotiated with ISIS, $50 \mathrm{mV}$ is the current trip limit and constitutes a good baseline trip-level to use for initial MICE operation once the target and beamline are fully operational [90]. The MICE experiment will sample from the beam just before extraction to produce suitable pion statistics and energies to match its goals. Hence the analysis of data was restricted to the last $2 \mathrm{~ms}$ of the spill with the proton kinetic energy close to the nominal $800 \mathrm{MeV}$. The statistics were split into samples with the same target conditions (see Table 6.8). For each of these conditions the mean number of coincident hits in the last $2 \mathrm{~ms}$ were calculated as was the mean of the sum of beam loss over this same period. This gives the average total beam loss measured in the last $2 \mathrm{~ms}$ for each target condition. A summary of this is shown in Table 6.9

The relationship between the two can be seen for both unshielded and shielded detectors in Figures 6.17 and 6.18 respectively. A linear least-squares fit is shown on each plot. The parameters estimated from these fits show the relationships between beam loss and particle 


\begin{tabular}{|c|c|c|c|}
\hline Target trajectory & Runs & Coincidences (shielded) & Coincidences (unshielded) \\
\hline \hline Dip 0.0082s & 3 & 0 & 0 \\
Dip 0.125s & 5 & 12 & 12 \\
Dip 0.130s & 3 & 8 & 6 \\
Dip 0.135s & 16 & 126 & 164 \\
Dip 0.140s & 4 & 81 & 97 \\
Dip 0.145s & 5 & 179 & 221 \\
\hline
\end{tabular}

Table 6.8: Accumulated coincident PMT hit statistics between 8-10ms

\begin{tabular}{|c|c|c|}
\hline Mean hits (shielded) & Mean hits (unshielded) & Mean beamloss $(\mathrm{V})($ Error $<1 \%)$ \\
\hline \hline 0 & 0 & 0.00337 \\
2.4 & 2.4 & 0.00717 \\
2 & 1.33 & 0.00387 \\
7.875 & 10.25 & 0.0153 \\
20.25 & 24.25 & 0.0273 \\
35.8 & 44.2 & 0.0487 \\
\hline
\end{tabular}

Table 6.9: Mean data between 8-10ms for both shielded and unshielded detectors

yield summarised in Table 6.10. One should also notice the $0.05 \mathrm{~V}$ level which is the current ISIS trip limit.

These linear relationships are particularly useful because the particle number density scales linearly up to the MICE acceptance. Therefore one can scale the number of coincident hits to estimate the number of recorded particles into the MICE acceptance as a function of beam-loss.

The flux of recorded singles into the MICE angular acceptance as a function of beam loss can be seen in Figures 6.19 and 6.20 with the results of linear fits to this data again summarised in Table 6.10.

\begin{tabular}{rl|}
\hline Singles into detector $(\mathrm{US})=$ & $951( \pm 106) \times$ beam loss S7 $-3.21( \pm 0.36)$ \\
Singles into detector $(\mathrm{S})=$ & $778( \pm 96) \times$ beam loss S7 $-2.6( \pm 0.32)$ \\
Singles into MICE acceptance $(\mathrm{US})=$ & $2.98( \pm 0.33) \times 10^{6} \times$ beam loss S7 $-1.0( \pm 0.1) \times 10^{5}$ \\
Singles into MICE acceptance $(\mathrm{S})=$ & $2.28( \pm 0.30) \times 10^{6} \times$ beam loss S7 $-7.7( \pm 1.0) \times 10^{3}$
\end{tabular}

Table 6.10: Results of linear least square fits to the number of particles detected in both the shielded and unshielded detectors as a function of total beam loss between 8-10ms. Also detailed are the results from similar fits extrapolating the detector results to the entire MICE beamline angular acceptance. (S) denotes value calculated for the shielded detector and (US) for the unshielded detector

From an extrapolation from the linear fit for the number of unshielded singles into the MICE 
acceptance, the maximum singles flux in the last $2 \mathrm{~ms}$ into the MICE acceptance achievable at the current beam loss trip limit is $3.0( \pm 0.5) \times 10^{4}$. This assumes a total of $50 \mathrm{mV}$ beam-loss in the last $2 \mathrm{~ms}$.

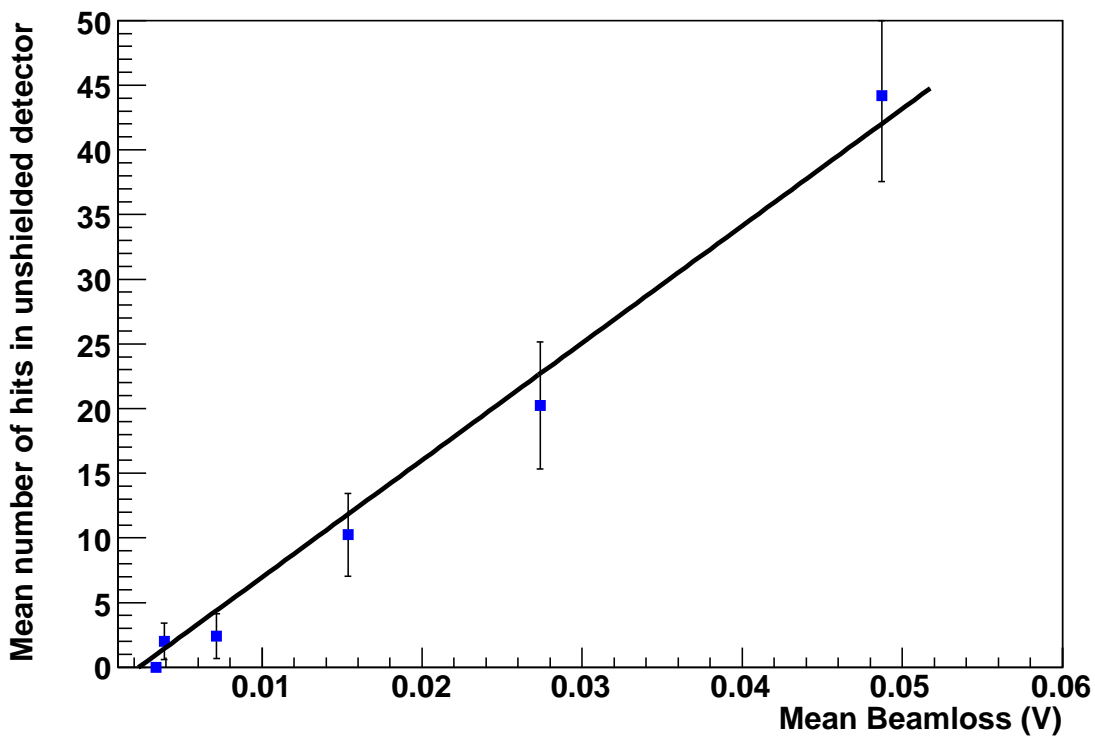

Figure 6.17: Relationship between number of coincident hits recorded in the unshielded detectors and the mean beam-loss in ISIS superperiod 7 monitors.

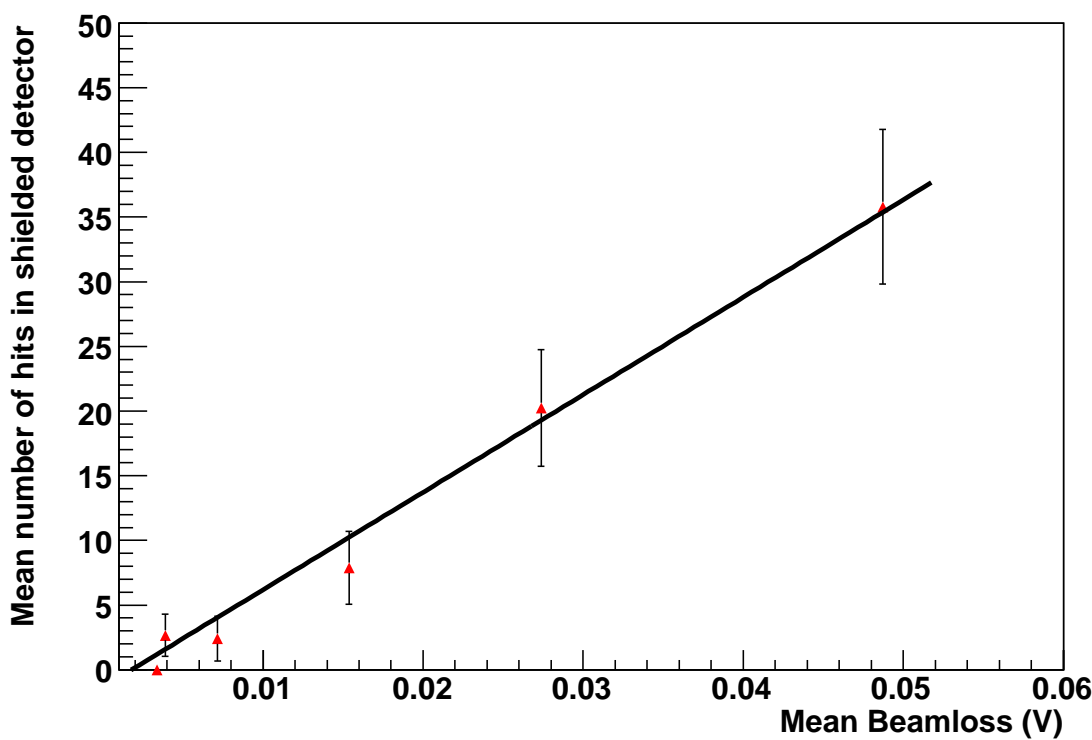

Figure 6.18: Releationship between number of coincident hits recorded in the shielded detectors and the mean beam-loss in ISIS superperiod 7 monitors. 


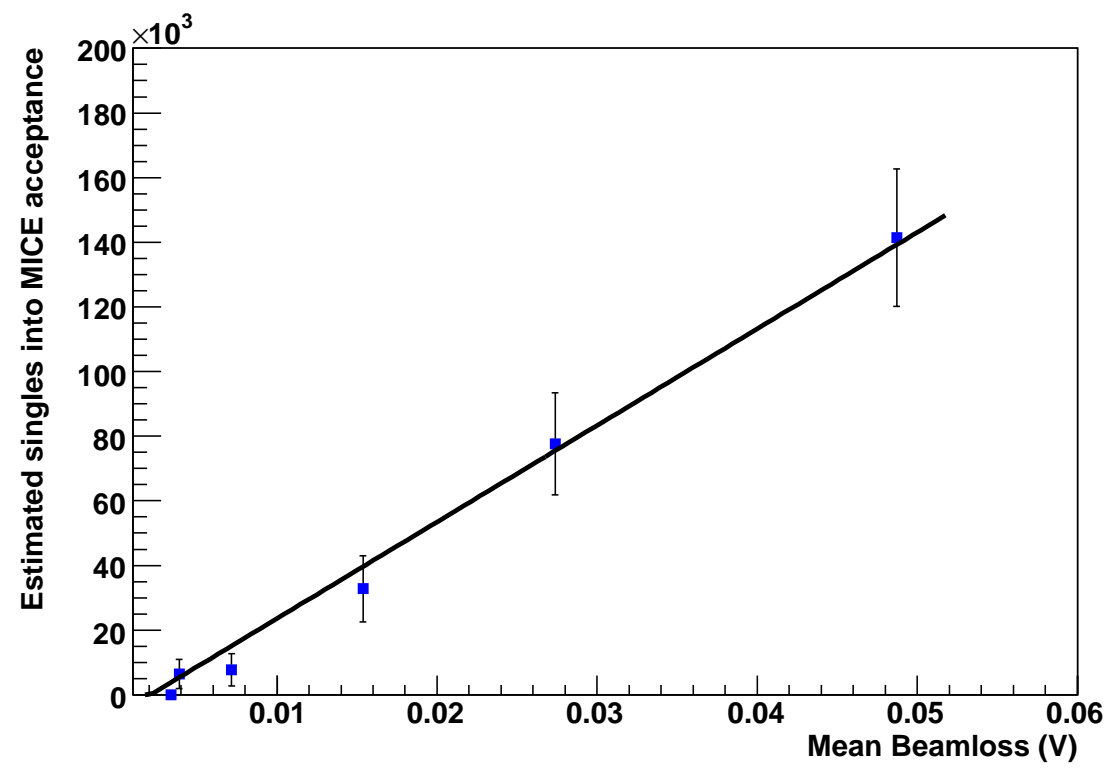

Figure 6.19: Estimation of the number of single particles into the MICE angular acceptance. The result is extrapolated from the data collected by the unshielded detector

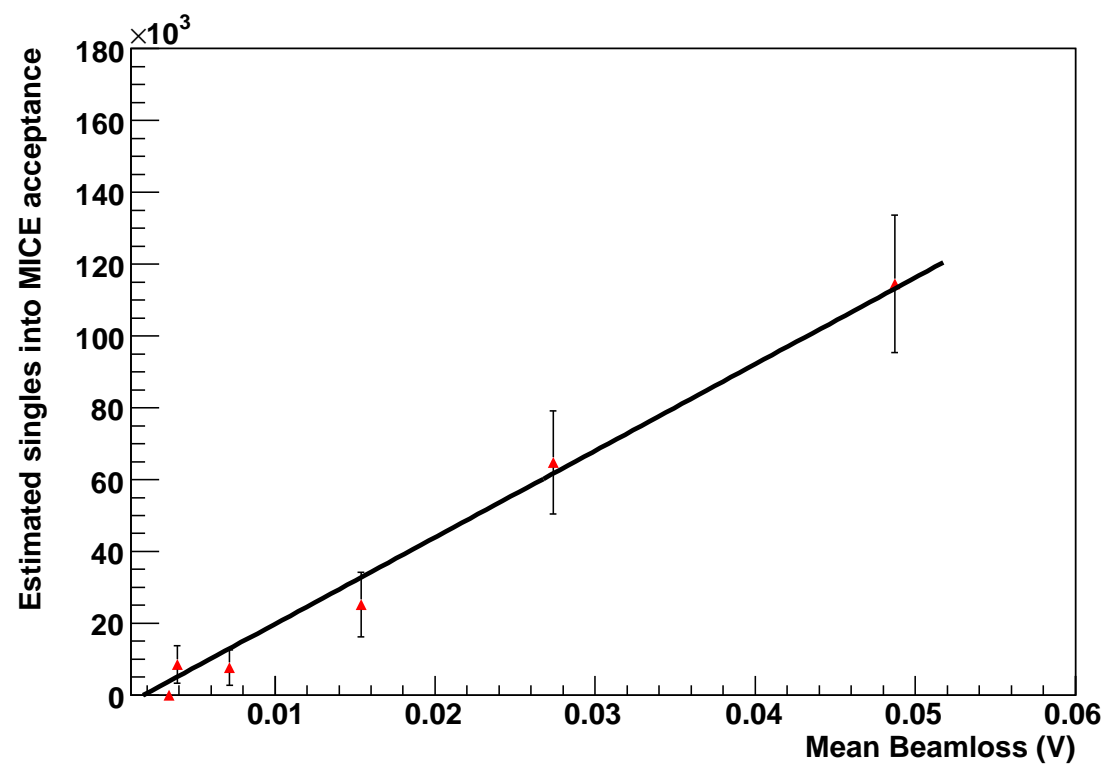

Figure 6.20: Estimation of the number of single particles into the MICE angular acceptance. The result is extrapolated from the data collected by the shielded detector 


\subsubsection{Comparison of singles per proton on target from simula- tion and data}

It is important to verify the GEANT4 and MARS simulations of the MICE target. It is crucial to compare the particle production yields at 25 degrees from the beam axis for both codes to those determined from data. From the target simulation discussed in the previous section it is possible to calculate the number of singles into the angular acceptance of our detectors per proton incident on the target. To calculate this from the data one must first calculate the mean number of hits in the last $2 \mathrm{~ms}$ for particles entering the MICE acceptance as a function of beam-loss, this has been calculated in the previous section and is summarised in Table 6.9. It is possible to calculate the associated number of protons on target to cause this beam loss if one assumes that all protons hitting the target are lost from the beam.

The calibration of individual beam loss signal was carried out by the ISIS team and was calculated by causing beam loss along the ring, measuring the reduction in the total ISIS current from the beam toroids and noting the signal change in each beam loss monitor [90]. The beamloss signal to incident proton flux conversion factor for these detectors for protons of energy 780 $\mathrm{MeV}(9 \mathrm{~ms})$ is $3.5 \times 10^{-14} \mathrm{Vs} /$ proton and at $800 \mathrm{MeV}(10 \mathrm{~ms}) 3.8 \times 10^{-14} \mathrm{Vs} /$ proton with a $50 \%$ error on these calibrations. Assuming the value at $9 \mathrm{~ms}$ this allows the numbers of interacting protons to be calculated for each beam loss value measured over the average $2 \mathrm{~ms}$ between 8 $10 \mathrm{~ms}$ of the spill. These can be seen in Table 6.11. Also shown in this table is the fraction of a bunch this loss corresponds to. The measured current for the test was $170 \mu A$, which corresponds to $2.1 \times 10^{13} /$ bunch. The error on the number of protons on target is dominated by the $50 \%$ calibration error abd by the assumption of the value of the calibration at $9 \mathrm{~ms}$ which is estimated to be $(3.8-3.5) / 3.5=8.6 \%$. The differing target dip conditions coupled to the shrinking on the beam as acceleration proceeds can cause the beam to "shrink" away from the beam only for the target to intercept again at a different time. This non-linear behaviour relating target delay and beam loss causes the range of values shown in Table 6.11. The numbers of singles should increase linearly with the beamloss as can be seen from the previous section.

The number of singles into the detector acceptance per proton on target can be calculated from both tracking codes simply by dividing the number of non-neutron singles, after multiplication by the efficiencies previously shown, by $1 \times 10^{7}$, the number of protons on target in each 


\begin{tabular}{|c|c|c|c|}
\hline Target condition & Mean beamloss (V) & Protons on target & Fraction of ISIS bunch \\
\hline \hline DIP 0.0082 & 0.00337 & $1.93 \times 10^{8}$ & $9.2 \times 10^{-6}$ \\
DIP 0.125 & 0.00717 & $4.10 \times 10^{8}$ & $2.0 \times 10^{-5}$ \\
DIP 0.130 & 0.00387 & $2.22 \times 10^{8}$ & $1.1 \times 10^{-5}$ \\
DIP 0.135 & 0.0153 & $8.78 \times 10^{8}$ & $4.2 \times 10^{-5}$ \\
DIP 0.140 & 0.0273 & $1.56 \times 10^{9}$ & $7.4 \times 10^{-5}$ \\
DIP 0.145 & 0.0487 & $2.78 \times 10^{9}$ & $1.3 \times 10^{-4}$ \\
\hline
\end{tabular}

Table 6.11: The number of protons lost from the ISIS beam as an absolute number and as a fraction of the total number of protons in each bunch. The values calculated correspond to the number of protons on target for the range of dipped target settings.

simulation. This was calculated from the GEANT4 and MARS Monte Carlo in addition to the detector data. To calculate the number of singles per p.o.t from the data one chooses the target condition which gives the greatest mean number of hits in the detector (i.e. DIP 0.145s). This number is then divided by the corresponding mean number of protons on target calculated from the mean. The fractional error in this quantity is given simply by the addition in quadrature of the fractional errors in the number of incident singles detected and the fractional error in the assumption of the calibration value at $9 \mathrm{~ms}$. The first error is purely a $\sqrt{N}$ Poissonian error and the latter was mentioned previously and is the combination of the statistical errors given by the intrinsic error in the calibration signal and the estimation of the calibration value at $9 \mathrm{~ms}$ given by $\sqrt{0.5^{2}+0.086^{2}}$. The error in the values quoted for both simulations are purely due to the error on the number of singles detected and are again Poissonian.

The results are shown in Table 6.12. The results show agreement well within errors and although this experiment does not have sufficient accuracy to comment on which of the codes is the most accurate it does validate the results from both codes and provides confidence that the measurement and analysis techniques are correct and that the simulations can be used to calculate normalised rates in the MICE beamline which will be discussed more fully in Chapter 7. 


\begin{tabular}{c|c|c} 
& Singles $/$ p.o.t $($ Unshielded $)\left(\times 10^{-8}\right)$ & Singles $/$ p.o.t $($ Shielded $)\left(\times 10^{-8}\right)$ \\
\hline MARS & $1.70( \pm 0.10)$ & $1.52( \pm 0.096)$ \\
GEANT4 & $2.47( \pm 0.12)$ & $1.61( \pm 0.010)$ \\
DATA & $1.59 \pm 0.24$ (stat) \pm 0.81 (sys) & $1.29 \pm 0.22$ (stat) \pm 0.65 (sys)
\end{tabular}

Table 6.12: The calculated number of non-neutron singles into the $1 \mathrm{~cm}^{2}$ detector acceptance using MARS, GEANT4 and data. Results are shown for both shielded and unshielded detector cases.

\subsubsection{Studies of particle production into the MICE beamline as a function of target position}

It is important to try to ascertain how the beam loss to particle production relationship already established behaves in relation to the position of the MICE target. No information exists on the ISIS beam profile in the region of the target and hence information can only be extracted from the beam loss signal recorded as to the interaction of the target with the beam.

The target position was recorded by the DAQ as an analogue voltage signal provided by the Sheffield target group [89]. This was converted to a spatial dimension giving the distance from the central ISIS beam axis to within $\pm 75 \mu \mathrm{m}$ [91]. The mean target depth in the last $2 \mathrm{~ms}$ for each of the different run conditions were calculated and are summarised in Table 6.13. The error introduced by this averaging process is estimated by looking at raw data histograms to be approximately $\pm 1.4 \mathrm{~mm}$. Figure 6.21 shows the mean beam loss as as function of depth.

\begin{tabular}{|c|c|c|}
\hline Target trajectory & Mean target depth $(\mathrm{mm})$ & Mean total beam loss (V) \\
\hline \hline Dip 0.0082s & 54.5962 & 0.003373 \\
Dip 0.125s & 43.7034 & 0.007117 \\
Dip 0.130s & 42.6233 & 0.00389 \\
Dip 0.135s & 41.6260 & 0.01537 \\
Dip 0.140s & 40.6216 & 0.02737 \\
Dip 0.145s & 39.6343 & 0.04870 \\
\hline
\end{tabular}

Table 6.13: Mean target depth and mean total beam loss between 8-10ms

In addition, the relationship between the number of singles into the MICE acceptance has already been established, hence by using this scale factor, the number of recorded singles into the MICE acceptance can be estimated. The parameters obtained by an exponential fit to this 
data is calculated and the relationship is:

Singles into MICE acceptance $=\exp ^{40.78\left( \pm 1 \times 10^{-3}\right)-0.73\left( \pm 3 \times 10^{-5}\right) \times \text { Disp }}$

where Disp is the target distance from the ISIS beam centerline.

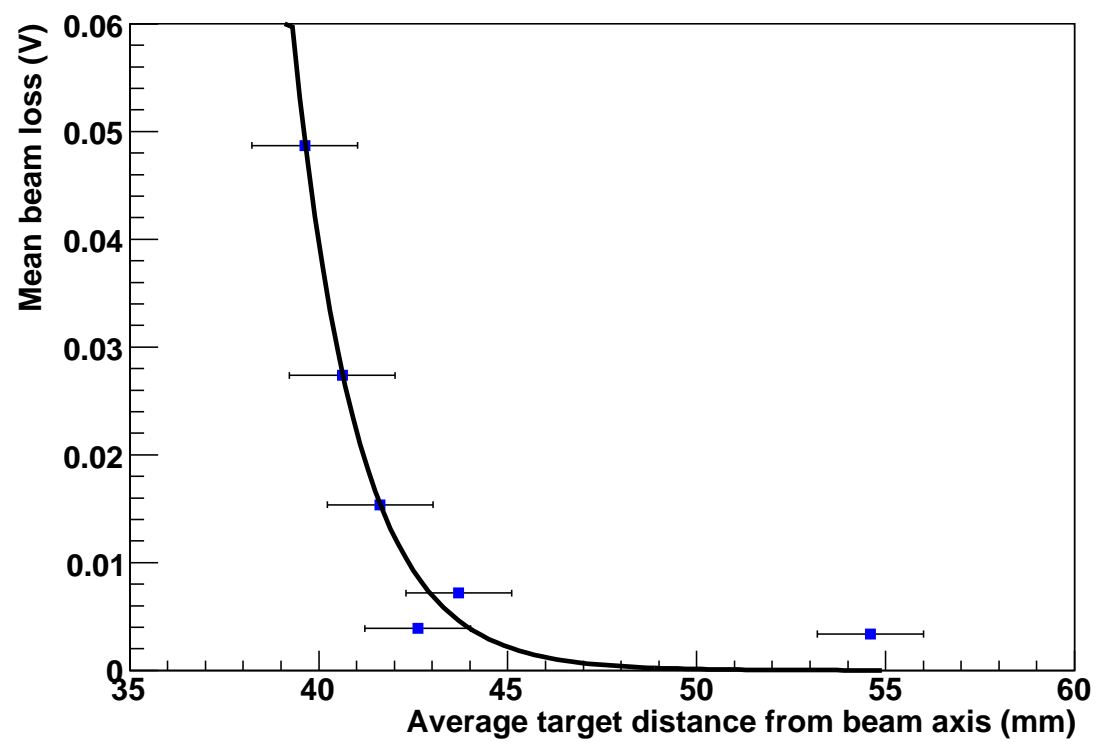

Figure 6.21: The total beam loss in the last $2 \mathrm{~ms}$ as a function of the mean target depth between $8-10 \mathrm{~ms}$

\subsection{Conclusions}

The target test detailed here is the first in situ test of a MICE target in ISIS. No data was previously available of ISIS beam conditions in the vicinity of the target. As will be shown in Chapter 7, estimations for particle rates in the MICE beamline are completely determined by normalisation factors calculated from GEANT4 and MARS tracking codes. These codes have been shown to be in agreement with the data accumulated during this experiment, with the numbers of singles/p.o.t agreeing within the precision of the data taken.

Another important consideration for MICE running is losses in ISIS. Again the relationship between beam loss, target insertion and particle yields into the MICE acceptance has never been studied. It is crucial that this be understood to sufficient precision because both the target 


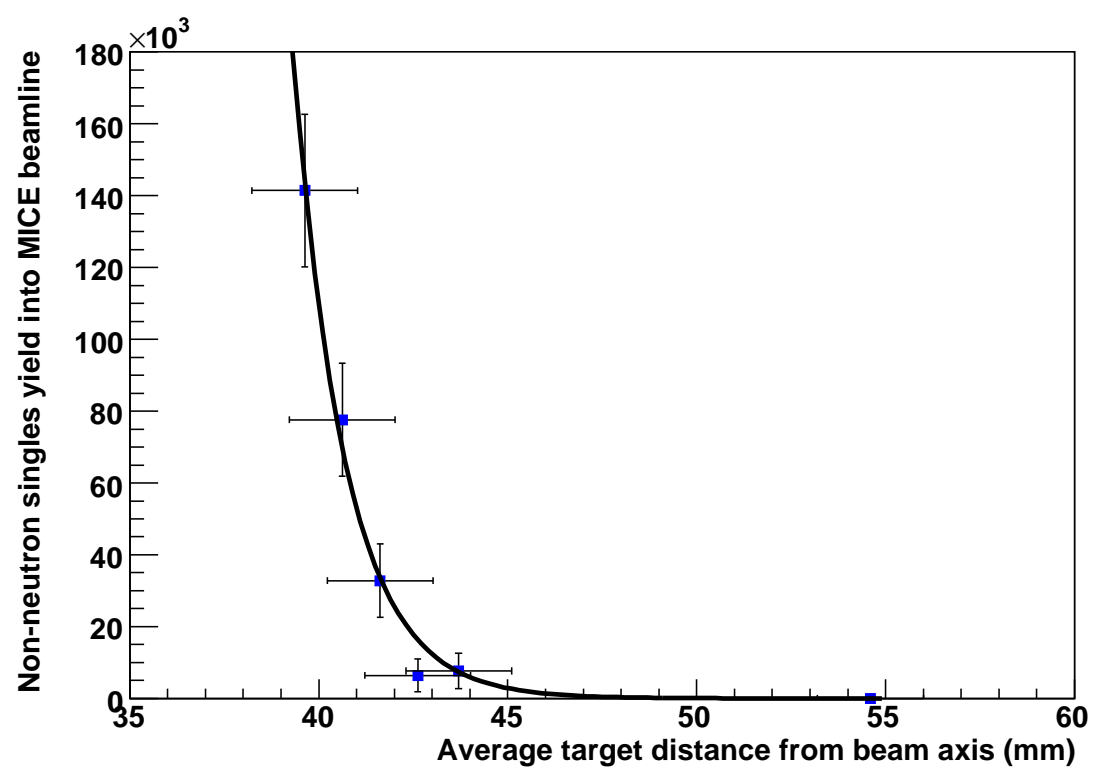

Figure 6.22: Estimation of the number of recorded singles into the MICE angular acceptance as a function of the mean target depth between $8-10 \mathrm{~ms}$. The result is extrapolated from the linear fit to data collected by the unshielded detector and scaled to the MICE angular acceptance

operation and ISIS losses will have the largest effect on the flux of muons available for MICE physics. In determining these relationships one now has more detailed knowledge of coupling to ISIS. Limits have been put on the maximum number of singles into the MICE beamline assuming the current ISIS trip limit and the corresponding worst case target conditions that would achieve this. The transverse ISIS beam profile in the $600-800 \mathrm{MeV} / \mathrm{c}$ range at the location of the MICE target has also been measured. Generally, when calculating particle rates along the beamline and through MICE, assumptions have been made as to the number of protons intercepted by the target $(0.14 \%$ of beam). It is important to note however that this value is greater than the values determined from the target test (see Table 6.11). Undoubtedly for MICE running the beam loss limit will need to be modified as the current limit is consistent with no expected beamloss in super-period 7. From the work shown in this chapter one can estimate the level of ISIS loss which will provide the muon rate desired for MICE and again this will be discussed in Chapter 7. 


\section{Chapter 7}

\section{MICE Beamline Design}

\subsection{Introduction}

To achieve the MICE physics goals the experiment must be supplied with a reliable and adaptable supply of both muon charge conjugates. This requirement will be fulfilled by the MICE beamline [92]. The MICE beamline replaces the old ISIS HEP beamline and will supply beam to the MICE experiment which is housed in ISIS Hall 5.2 as shown in Figure 7.1. The beamline is based on a typical pion-muon beamline with important additional particle identification and beam preparation responsibilities. This chapter will discuss the design and evaluation of this beamline.

\subsection{Goals for the beamline}

The cooling requirements in Neutrino Factory will no doubt be subject to a cost optimisation. The severity of the cooling required is intimately linked to the accelerator scheme being used. Beam emittances in the range 30-45 $\pi \mathrm{mm}$ mrad are regularly discussed. The baseline for the MICE experiment is not to show cooling in beams as large as this but the scalability of MICE will enable the study of larger beams.

To study cooling of a variety of different phase spaces MICE should measure muon cooling of a variety of muon beams. Muon cooling performance should be measured for a number of beams of varying momenta and emittance. This will allow detailed simulation and evaluation 


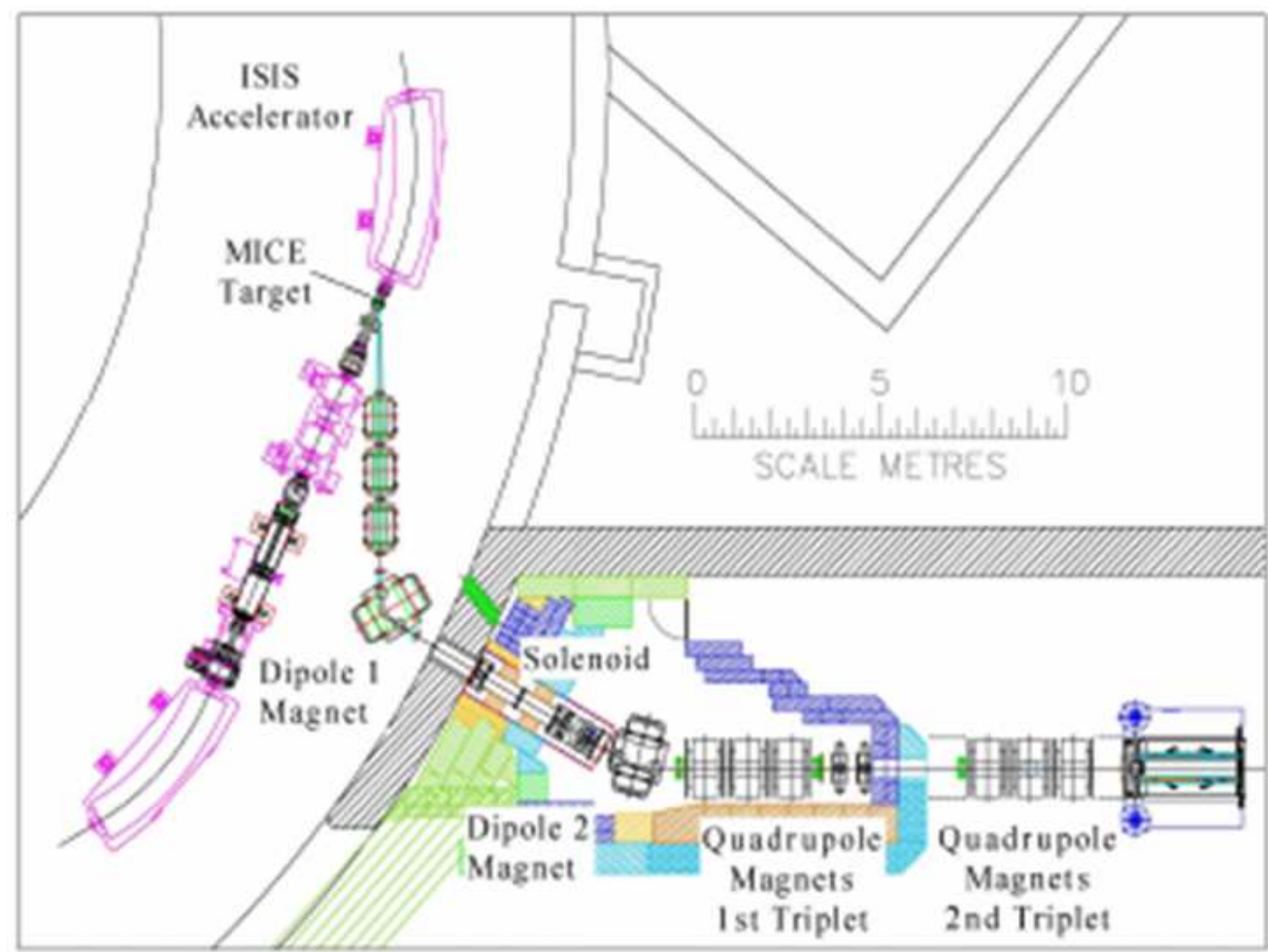

Figure 7.1: The location of the MICE experiment and beamline in relation to ISIS

of Neutrino Factory muon front ends and a realistic optimisation of the cooling channel and storage ring performance.

The MICE beamline should be able to provide a minimum of 9 different matched muon beams to MICE. The baseline requirement is to provide matched muon beams at three different emittances. The values are $1,6,10 \pi \mathrm{mm} \mathrm{mrad}$, although these are primarily guides. The important feature is that beams should be produced which span the $1 \pi \rightarrow 12 \pi \mathrm{mm}$ mrad range of emittances. The cooling performance of MICE should be also quantified for muon beams over a range of momenta. To facilitate this the beamline should provide, for every emittance case, beams at three momenta, 140, 200 and $240 \mathrm{MeV} / \mathrm{c}$. As the experiment is designed to be particle-by-particle it is possible to use this cooling data to artificially construct beams of arbitrary emittance using software, providing one is careful not to have depleted populations of phase space. 


\subsection{The MICE target}

The MICE target is a small titanium fin which supplies particles to the MICE beamline upon insertion into the ISIS as was described in the previous chapter. This is a new target system which will replace the old target system and should be able to operate at a pulse rates of a few $\mathrm{Hz}$ with good depth reproduceability and high acceleration so as to dip into the ISIS beam in the last millisecond of the ISIS acceleration cycle. The titanium fin samples the edge of the ISIS beam, which aids in keeping the beam-loss caused by the target minimal such that it meets stringent constraints imposed by ISIS. Timing of the insertion will be delayed so that it matches to the synchrotron cycle such that the target will be inserted at the end of a pulse where the proton kinetic energy is $800 \mathrm{MeV}$. The target timing will also be synchronised to the RF phase in MICE accelerating cavities. A more thorough description of the ISIS target was given in Chapter 6.

\subsection{MICE target normalisation}

To understand the performance of the beamline it is important to simulate the interaction of the titanium in the proton beam. Evaluation of particle yields into the MICE acceptance is important to estimate beam rates which have an impact on beamline design, detector design and formulation of a run plan for the experiment.

The MICE beamline group have simulated the target using three independent particle production codes MARS [52], GEANT4 [51] and LAHET [93]. In each of these simulations $1 \times 10^{7}$ protons were incident on a titanium target with dimensions given in Table 7.1. In the LAHET and MARS simulations particles crossing a spherical boundary centred on the target are recorded. The LAHET simulation contains only $\pi^{+}$where as the MARS simulation contains all particles crossing this boundary. The GEANT4 (using the LHEP binary cascade hadronic engine) simulation samples all particles crossing a circular surface with a $5 \mathrm{~m}$ radius located $9.5 \mathrm{~mm}$ downstream from the centre of the target except for a $2 \mathrm{~mm}$ radius disk directly along the proton beam axis. The momentum distribution for particle yields can be seen in Figures 7.2 and 7.3. In this energy range spallation neutrons and pions are producted in the following reactions: 


$$
\begin{aligned}
p+p & \rightarrow p+n+\pi^{+} \\
& \rightarrow p+p+\pi^{0} \\
p+n & \rightarrow p+n+\pi^{0} \\
& \rightarrow p+p+\pi^{-} \\
& \rightarrow n+n+\pi^{+}
\end{aligned}
$$
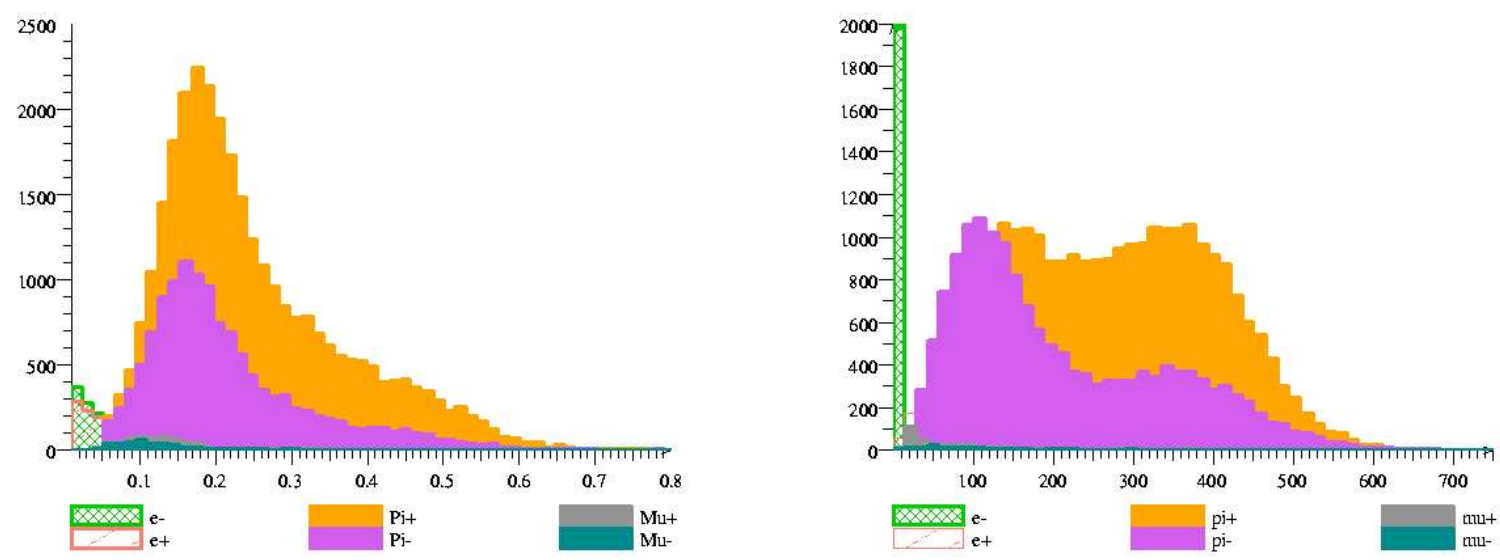

Figure 7.2: Particle production histograms as a function of momentum showing meson and lepton production from MARS (left panel) and GEANT4 (right panel).

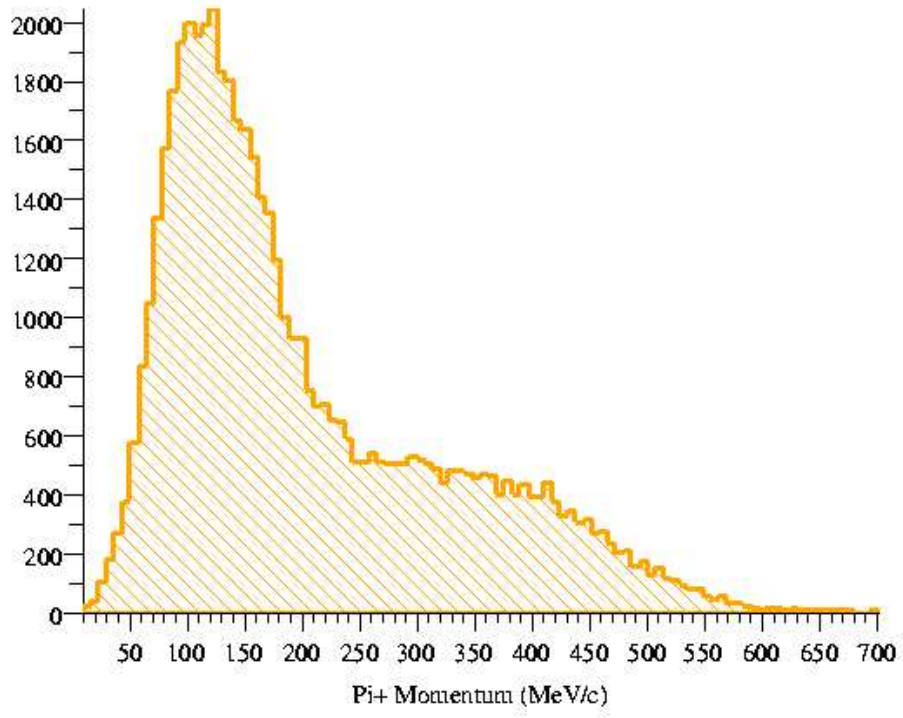

Figure 7.3: The $\pi^{+}$momentum distribution from a LAHET simulation of $800 \mathrm{MeV}$ protons on a titanium target 
When simulating the beamline it is normal to track with an initial Gaussian beam of pure $\pi^{+}$from the target. The angular distribution of the initial beam is determined to be larger than the angular acceptance of the first quadrupole aperture in the capture channel. The mean momentum of the pion beam is part of the tune and is tailored such that the desired momentum condition at the reference point in the upstream hydrogen absorber in MICE is achieved. For the nominal tune condition this value is $200 \mathrm{MeV} / \mathrm{c}$ which corresponds to a mean momentum of approximately $208 \mathrm{MeV} / \mathrm{c}$ in the upstream tracker. It is crucial to be able to couple this artificial pion beam to what is expected from the target. This will enable normalisation of the rates measured in the beamline simulations. To do this, the target simulation sampled distributions are weighted into the MICE angular and momentum acceptance. The polar angle of each particle is calculated and its weight is computed as its fraction of $2 \pi$ in azimuth that the beamline accepts. These weights are then summed for each different particle species.

\begin{tabular}{|c|c|}
\hline Target Material & Titanium \\
Target Height & $10 \mathrm{~mm}$ \\
Target height intersecting ISIS proton beam & $2 \mathrm{~mm}$ \\
Target Width & $1 \mathrm{~mm}$ \\
Target length parallel to beam & $10 \mathrm{~mm}$ \\
Fraction of an interaction length & 0.036 \\
ISIS beam area at target & $4400 \mathrm{~mm}^{2}$ \\
ISIS beam density at target (edge) & $10 \%$ of maximum beam density \\
Target insertions per second & 1 \\
Target duration in beam in good RF & $2.5 \times 10^{13}$ \\
ISIS protons per bunch & $1.5 \mathrm{MHz}$ \\
Bunch rate at target & $1.7 \times 10^{12}$ \\
\hline
\end{tabular}

Table 7.1: Detailed simulation parameters in modeling the MICE target and determining normalisations to estimate tertiary beam rates

Using the assumptions stated in Table 7.1 one can then calculate the absolute rate of pions along the beamline according to Equations (7.1), (7.2):

$$
\pi^{+} \text {intersecting target per second }(\xi)=\frac{\rho \times \text { Weighted number of } \pi^{+}}{\text {Protons on target }}
$$

where $\rho$ is the number of protons intersecting the target per $1 \mathrm{~ms}$ 


$$
\pi^{+} \text {rate }=\frac{\text { Number of tracked singles at downstream location }}{\text { Initial number of tracked pions } \times \xi}
$$

The weighted number of $\pi^{+}$was calculated for each of the three $200 \mathrm{MeV} / \mathrm{c}$ beamline tunes that will be presented at the end of the chapter. The number of pions into the MICE acceptance is then calculated for the LAHET, GEANT4 and MARS target distributions. A summary of this information is given in Table 7.2. This will be used to normalise beamline rates in the analysis sections.

\begin{tabular}{|c|c|c|c|}
\hline Beamline Tune & $\begin{array}{l}200 \mathrm{MeV} / \mathrm{c}, \quad 1 \pi \\
\mathrm{mm} \operatorname{mrad}\end{array}$ & $\begin{array}{l}200 \mathrm{MeV} / \mathrm{c}, 7.1 \pi \\
\mathrm{mm} \mathrm{mrad}\end{array}$ & $\begin{array}{l}200 \mathrm{MeV} / \mathrm{c}, \quad 10 \pi \\
\mathrm{mm} \mathrm{mrad}\end{array}$ \\
\hline $\begin{array}{l}\pi^{+} \text {central momentum } \\
(\mathrm{MeV} / \mathrm{c})\end{array}$ & 423.28 & 444.71 & 453.17 \\
\hline $\begin{array}{l}\text { Momentum acceptance } \\
\text { of beamline }(\mathrm{MeV} / \mathrm{c})\end{array}$ & $406.28 \rightarrow 440.28$ & $427.71 \rightarrow 461.71$ & $436.17 \rightarrow 470.17$ \\
\hline $\begin{array}{l}\text { Number of } \pi^{+} \text {into } \\
\text { MICE acceptance } / 10^{7} \\
\text { protons (LAHET) }\end{array}$ & 3.056 & 3.237 & 3.034 \\
\hline $\begin{array}{l}\text { Number of } \pi^{+} \text {into } \\
\text { MICE acceptance } / 10^{7} \\
\text { protons (MARS) }\end{array}$ & 3.882 & 2.788 & 2.498 \\
\hline $\begin{array}{l}\text { Number of } \pi^{+} \text {into } \\
\text { MICE acceptance } / 10^{7} \\
\text { protons (GEANT4) }\end{array}$ & 3.659 & 3.619 & 3.580 \\
\hline $\begin{array}{l}\text { Pions/sec into MICE } \\
\text { beamline acceptance } \\
\text { (LAHET) } \times 10^{5}\end{array}$ & 5.19 & 5.50 & 5.15 \\
\hline $\begin{array}{l}\text { Pions/sec into MICE } \\
\text { beamline acceptance } \\
(\mathrm{MARS}) \times 10^{5}\end{array}$ & 6.59 & 4.73 & 4.24 \\
\hline $\begin{array}{l}\text { Pions/sec into MICE } \\
\text { beamline acceptance } \\
\left(\text { GEANT4) } \times 10^{5}\right.\end{array}$ & 6.21 & 6.14 & 4.74 \\
\hline
\end{tabular}

Table 7.2: Normalisation parameters for $200 \mathrm{MeV} / \mathrm{c}$ beams over a range of emittances

\subsection{Description of the beamline}

The MICE beamline can naturally be broken down into two distinct regions. The pion decay channel and muon matching section. A 3D representation of the pion channel created using 
Open GL [94] can be seen in Figure 7.5. The target produces significant numbers of pions with a $200-500 \mathrm{MeV} / \mathrm{c}$ momentum range as can be seen from a simulation of $800 \mathrm{MeV}$ protons on a titanium target shown in Figure 7.3. The pions produced are captured by the first quadrupole triplet. This triplet is located within the ISIS vault itself and is tuned for a large acceptance for pions. The three 20cm aperture Quad Type-IV magnets [95] transport the beam, which at this point is mainly protons and pions to the first of two dipole magnets [95] situated along the beamline. This large rectangular dipole is tuned to provide a $60^{\circ}$ horizontal bend into a solenoidal decay channel. The $\pi^{ \pm} \rightarrow \mu^{ \pm}$decay mainly happens within a $5 \mathrm{~m}$ long decay solenoid which was inherited from the Paul-Scherrer Institute (PSI), Switzerland. The beam pipe from the target up until the end of the decay solenoid is pumped to vacuum with aluminium vacuum windows at either end of the pipe. This vacuum is decoupled from the ISIS vacuum via a small air gap. The height of the ISIS proton beam is approximately $40 \mathrm{~cm}$ lower than the MICE beam height. To adjust for this, the first quad triplet is mounted on an inclined and tilted support as is the first dipole. This configuration can be seen in Figure 7.4. The magnets mentioned will be described in the following section.

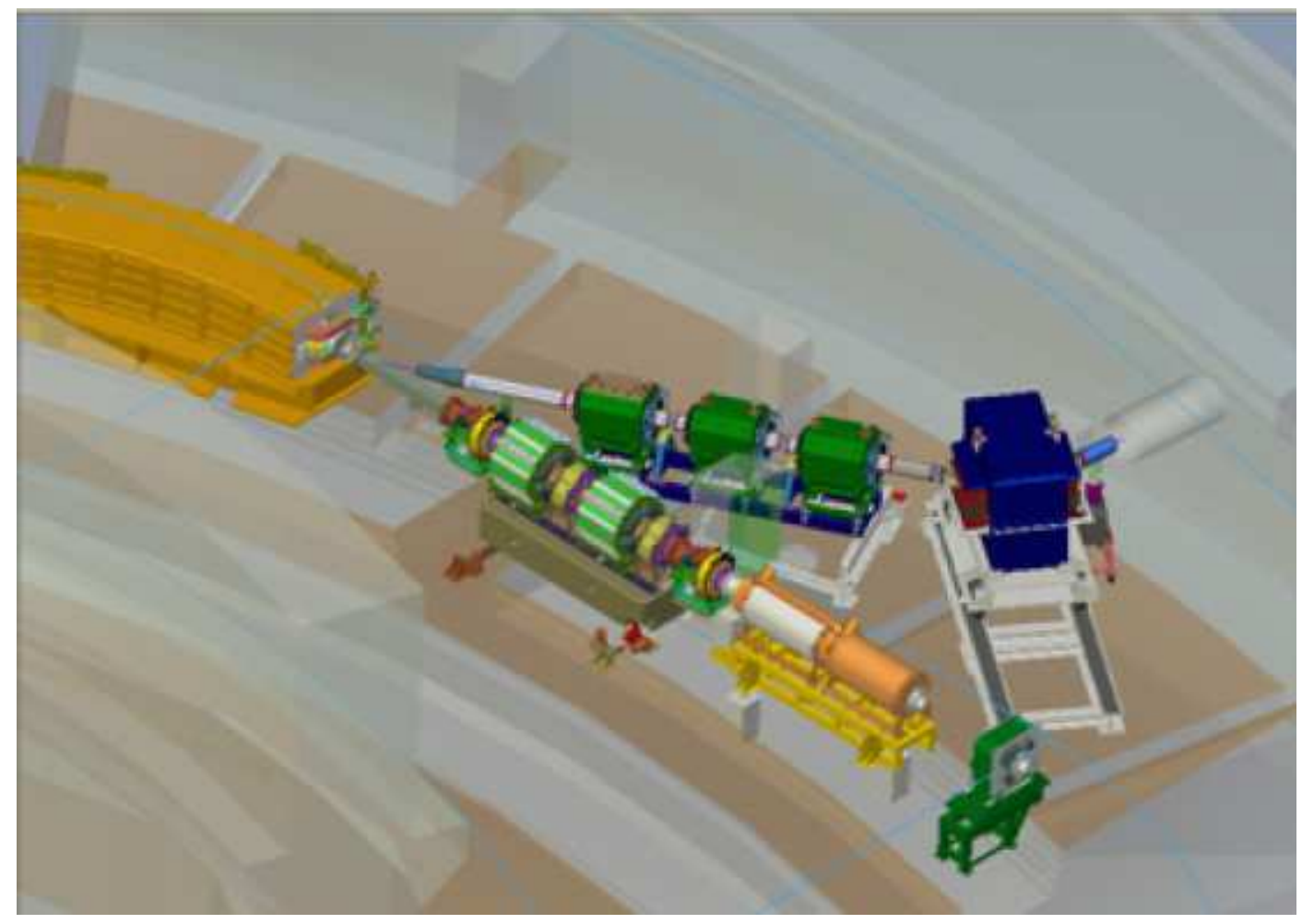

Figure 7.4: Engineering drawing showing the upstream section of the beamline inside the ISIS vault. The first quadrupole triplet and dipole can be seen placed on its raised support. 


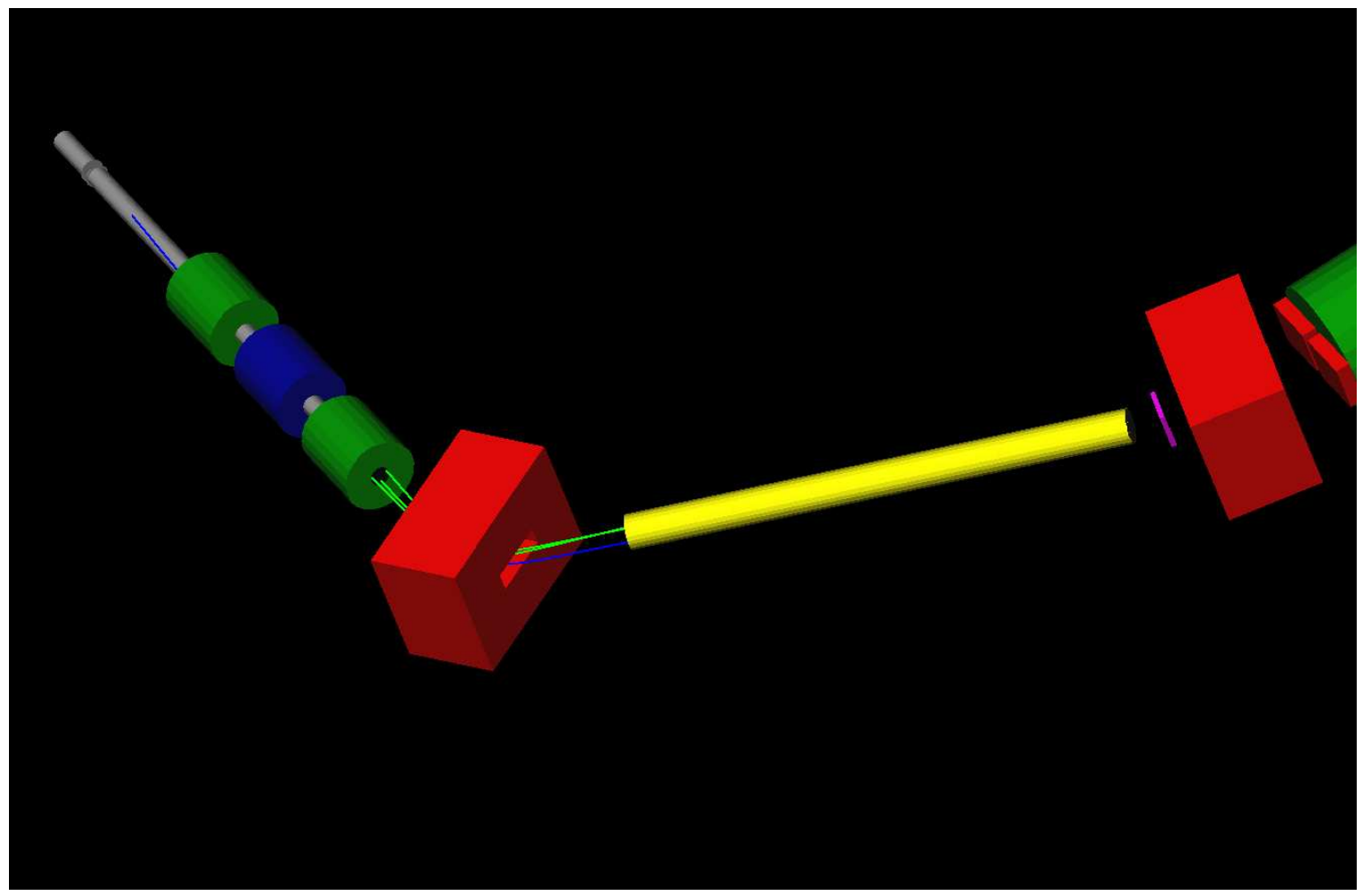

Figure 7.5: Open GL visualisation of the pion decay channel:- Upstream the grey vacuum pipe is shown as is the Type IV quad triplet. Both dipoles are shown (red) with the PSI decay solenoid (yellow) and proton absorber (cyan) between them

The beam exiting the decay solenoid still has a large proton population. To remove these a polyethylene volume of dimensions $40 \mathrm{~cm} \times 40 \mathrm{~cm}$ square by $5 \mathrm{~cm}$ thickness along the $\mathrm{z}$ axis is placed after a small drift space downstream of the decay solenoid. This proton absorber effectively removes all the protons from the beam. The beam then drifts to the second dipole in the beamline. The second dipole bends the beam into the MICE hall, tuned to provide a $30^{\circ}$ horizontal deflection (see Figure 7.5).

There is still significant pion contamination in the beam after passing through the polyethylene absorber and the second dipole is very effective as a muon selector. The kinematics of pion decay means muons with a decay in the backwards hemisphere in the centre of mass (backward muons) will have a significantly lower momentum than the remaining pions. One can map the reference pion momenta to a reference backward muon momentum such as is shown in Figure 7.6. The dipole is tuned to the backward muon momentum and as such these are preferentially transported with most of the pions remaining in the beam hitting the dipole or not falling within the subsequent quadrupole acceptance. 


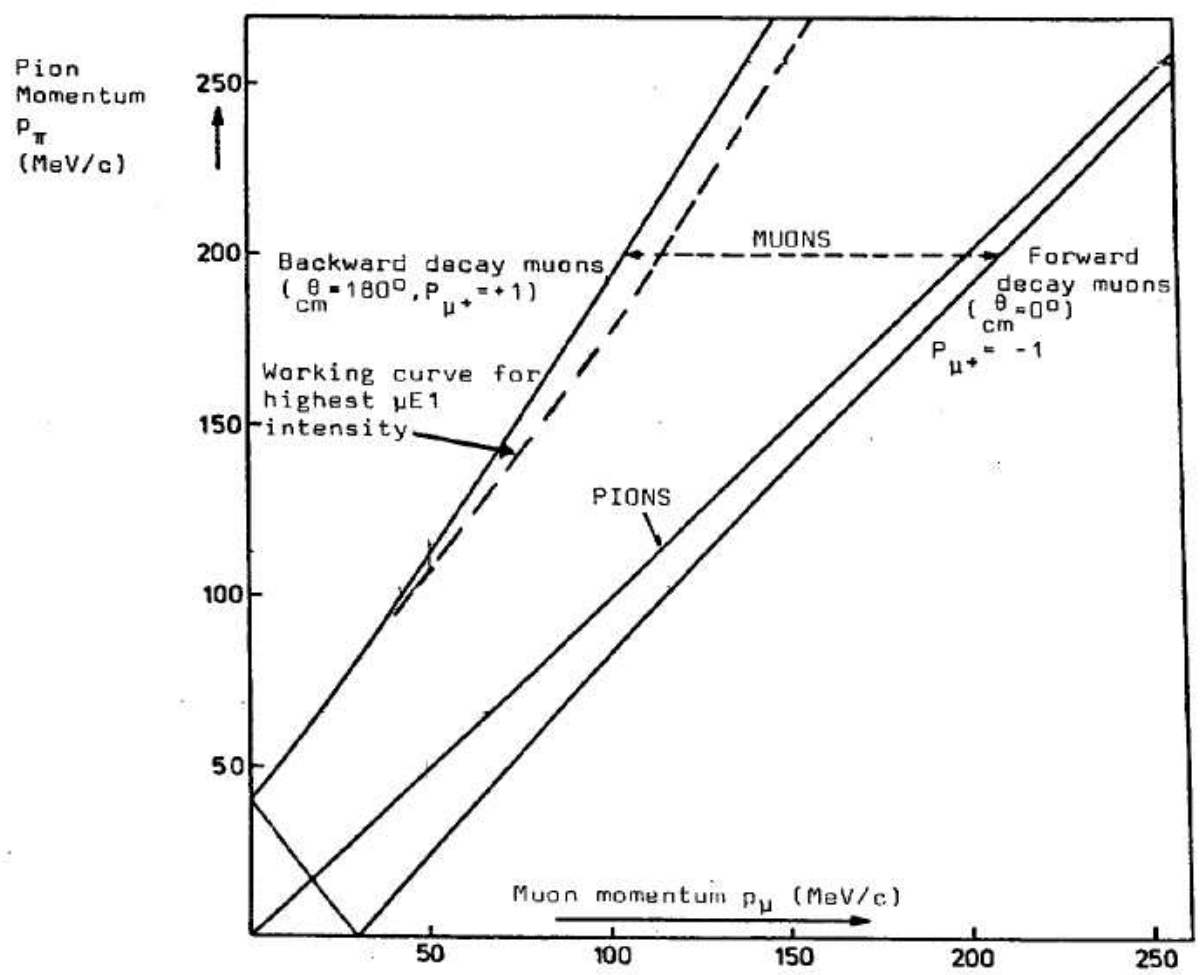

Figure 7.6: Relationship between the reference pion and muon lattice central momenta [96]

The downstream section of the MICE beamline is concerned with the successful transport of a variable matched beam into the MICE experiment. This is generally referred to as the muon matching section and does contain some of the important PID detectors mentioned in Chapter 5, namely time-of-flight walls TOF0 and TOF1 and the beamline Cherenkov detector $(\mathrm{CKOV})$. The transport of the muon beam over the $\approx 12 \mathrm{~m}$ long matching section is achieved via a lattice produced by a further two quadrupole triplets. The quads used in the muon channel have a larger aperture $(34 \mathrm{~cm})$ than those used further upstream to transport pions $(20 \mathrm{~cm})$. These larger apertures are required to allow for optimising currents to transport large beams. In the current design the first TOF station is placed between both pairs of triplets with the CKOV units slightly downstream from this. The second TOF station is placed downstream of the last triplet. The last significant beamline element is a lead diffuser which is positioned at the very end of the MICE beamline and actually sits within the tracker solenoid bore. The diffuser is an important feature in producing a matched beam, particularly at high emittances. The matching of the $\beta$-function of the beam to the $\beta$-function of the MICE lattice is important to produce a stable beam with minimal oscillations around the design phase space. The thickness 
of the diffuser is a crucial design characteristic of the beamline and will be variable for different beamline tunes. The mechanism to deliver the different diffuser thicknesses to the beamline is based on a complex rotating carousel system [97] which allows a diffuser plate to be extracted from the tracker solenoid and a new one inserted in approximately 5 minutes.

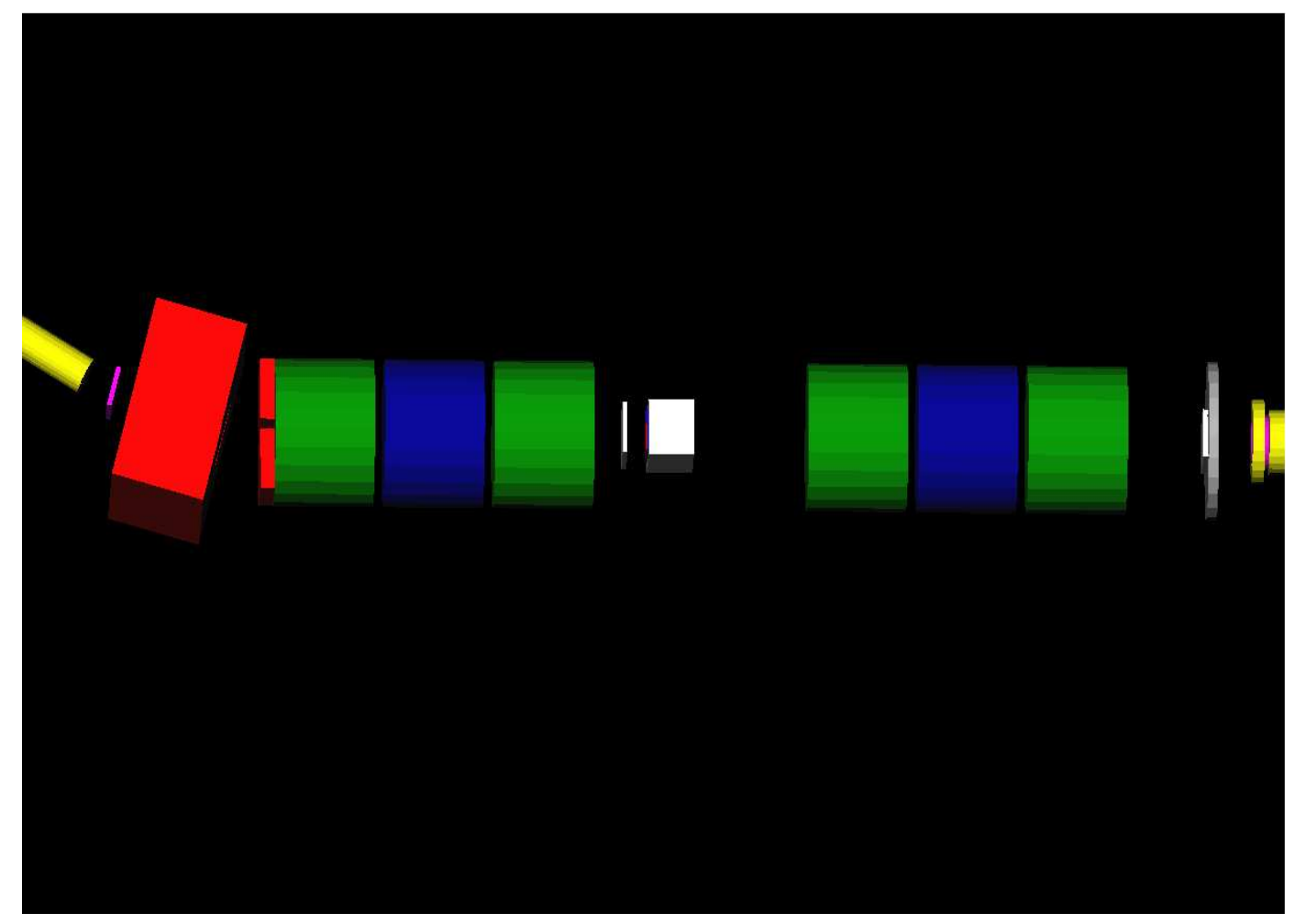

Figure 7.7: Visualisation of the muon matching section. Upstream one can see the downstream end of the decay solenoid (yellow), the proton absorber (cyan), the second dipole (large red). Also shown in this picture is horizontal collimation (red), this is not in the baseline but is currently being studied as a modification. Both sets of quad triplets are shown (green and blue volumes) as is TOF0 (thin white volume in centre), CKOV1 (larger white volume in centre) and TOF1 (thin white volume further downstream).

\subsection{Optical configuration of beamline}

The baseline transport lattices of the MICE beamline must be created using 12 magnets: 9 quadrupoles; 2 dipoles and one large solenoid. These are all reused magnets and are currently undergoing performance tests.

The quadrupoles used in the 3 triplets are from the NIMROD accelerator which operated at RAL during the 1950s/60s. Two different types are used in the MICE beamline, the upstream 
triplet constitutes three Type IV quads with both matching triplets using the Q35 quads [95]. The Type IV magnets have circular apertures of $101 \mathrm{~mm}$ radius. The apertures will be evacuated with an aluminium vacuum pipe passing though each quad in turn. The operating field gradients for this triplet change depending on the tune, with the maximum gradients of $10 \mathrm{~T} / \mathrm{m}$ never becoming a design issue. The beamline demands on these magnets are modest compared with the original design requirements for multi-GeV proton beams. The power to each quad will be provided by three separate $200 \mathrm{~A}, 30 \mathrm{~V}$ power supplies providing $6 \mathrm{~kW}$ each. The vacuum system for the pion capture section will be a UHV sealed volume pumped out using a single $500 \mathrm{l} / \mathrm{s}$ ISIS vacuum pump. The status of this vacuum will be controlled by the MICE control system which is based on the EPICS framework [98].

The two dipoles that will be installed are large 17 ton rectangular Type I NIMROD dipoles [95]. The nominal apertures for these magnets are rectangular with a 6" vertical gap. The first dipole must be tuned for a 60 degree horizontal rotation. This drives the magnet close to its nominal field saturation level (See Table 7.3) but provision exists to decrease the vertical aperture which allows for increased bend. The power supply for this magnet will operate at high current 500A, $240 \mathrm{~V}$ supplying $120 \mathrm{~kW}$. The beam is deflected into the matching section by a second dipole which is identical to the first but tuned for a 30 degree horizontal bend. This magnet is operated with more modest field requirements and as such requires a 200A, 100V supply providing $20 \mathrm{~kW}$ of power. These power supplies will be controlled via the EPICS framework. The large superconducting decay solenoid is included in the beamline design to capture the decay muons. This magnet was previously used in the $\mu \mathrm{E} 4$ beamline at PSI and is generally operated close to its maximum design field of $5 \mathrm{~T}$ to provide the greatest capture. The original control and power supply for this magnet will be used with interface to EPICS. A cryogenic system will provide liquid helium to the solenoid.

\subsection{Iteration of G4Beamline and TURTLE simula- tions}

The initial beamline design stage involves the use of the TRANSPORT [99]/TURTLE[100] design codes. These are both optics codes in which distribution transport theory drives beam 


\begin{tabular}{|l|l|l|l|}
\hline Beamline Magnet & $\begin{array}{l}\text { Physical length } \\
(\mathrm{cm})\end{array}$ & $\begin{array}{l}\text { Aperture size } \\
(\mathrm{cm})\end{array}$ & $\begin{array}{l}\text { Typ. Max required gra- } \\
\text { dient or field (limit in } \\
\text { brackets })\end{array}$ \\
\hline \hline Quad Type-IV & 110 & 20 & $1.6(10) \mathrm{T} / \mathrm{m}$ \\
Quad Type-35 & 117 & 34 & $2(8) \mathrm{T} / \mathrm{m}$ \\
Dipole Type1 4" & 140 & $\mathrm{H}: 66$ & $(1.7) \mathrm{T}$ \\
Dipole Type1 6" & 140 & $\mathrm{H}: 66$ & $1.5(1.47) \mathrm{T}$ \\
Dipole Type1 8" & 140 & $\mathrm{H}: 66$ & $0.4(1.25) \mathrm{T}$ \\
PSI Decay Solenoid & 500 & 11 & $5(5) \mathrm{T}$ \\
\hline
\end{tabular}

Table 7.3: Beamline magnets physical properties

propagation. TURTLE supports additional particle implementations such as particle decays. In linear transport theory, paraxial particle orbits can be expressed using the spatial distance from a central reference orbit and the divergence. One can express this as a 4-component trace-space vector denoted by (7.3).

$$
\bar{X}=\left[x, x^{\prime}, y, y^{\prime}\right]
$$

where $\mathrm{x}$ and $\mathrm{y}$ are the normal cartesian coordinates and $x^{\prime}$ and $y^{\prime}$ are the divergence angles for $\mathrm{x}$ and $\mathrm{y}$ respectively.

If transverse forces are linear we express the resultant effect of these forces on the radial vector $X$ as:

$$
X_{1}=\overline{\overline{M_{1}}} X_{0}
$$

where:

$\overline{\overline{M_{1}}}$ is a $4 \times 4$ matrix known as the transfer matrix. If the distribution is not subjected to any accelerating forces then this matrix has the property that:

$$
\operatorname{det} M=1
$$

This method can then be extended to many element optical systems, noting that the matrix multiplication operation is non-commutative. It can also be extended to transport beam distributions in which case the transfer matrix modifies the shape of the beam ellipse. As an 
illustration, a typical example is a transfer matrix for a thin lens with focal length (f). If one considers only the $\left(x, x^{\prime}\right)$ space the transfer matrix is given by:

$$
\overline{\overline{M_{1}}}=\left(\begin{array}{cc}
1 & 0 \\
1 / f & 1
\end{array}\right)
$$

This is the method in which the optics codes approximate a fringe field. The differences between this approximate method and the fringe field model used in the tracking code can lead to important differences in the evaluation of lattices. The thin lens transfer matrix shows that a thin-lens element supplies an angular kick proportional to the spatial displacement, but it does not affect the displacement.

The choice of using optics codes at this stage of design is primarily motivated by very fast iteration times. They do not track individual particles, and as such are not CPU intensive. There are certain criteria used to structure the tuning process and these will be explained briefly. Firstly, it should be noted that the design scope for the pion capture channel is fairly limited. The location of the triplets in the synchrotron vault is effectively frozen due to the geometry of the target/ring and the bore-hole into hall 5.2 for the decay solenoid. This requires that the first quadrupole can be no closer to the target than approximately $3 \mathrm{~m}$, with the distance to the centre of the first dipole $\approx 7.98 \mathrm{~m}$. Generally modification of the magnet optics are the only variable we have left to tune the capture section.

The design strategies for the muon matching section aim to keep the distance between the second dipole and the first subsequent quadrupole to a minimum such that the quad acceptance is as large as possible and to position PID detectors close to beam focii while keeping the TOF separation to an adequate level.

The matching and emittance generation of the muon beam into the tracker solenoid is achieved by modifying the beam size and scattering thickness of the lead beam degrader. The matching lattice is modified to focus a beam with an upright phase-space ellipse onto the lead diffuser. The $X_{r m s}$ and $X_{r m s}^{\prime}$ of the focussed beam will have a transverse beta function given by $(7.6)$.

$$
\beta_{\text {beam }}=\frac{X_{r m s}}{X_{r m s}^{\prime}}
$$

The transverse beta function for a solenoid is given by (7.7) [101]: 


$$
\beta_{\perp}=\frac{2 p}{B_{\text {sol }}}
$$

Thus, for the beam to be matched one requires these two expressions to be equal.

The emittance of the input beam $\left(\epsilon_{\text {input }}\right)$ must now be increased such that it achieves the desired emittance into MICE $\left(\epsilon_{\text {design }}\right)$. This is achieved by passing the beam through a variable thickness of lead. Inside this material the change in emittance is given by (7.8).

$$
\epsilon_{\text {design }}-\epsilon_{\text {input }}=\frac{1}{2} \beta_{\text {beam }}<\theta^{2}>
$$

The lattice and multiple scattering in the lead is altered until both the required emittance and beta function is achieved. Subsequently the design parameters from the optics code is then implemented in the G4beamline tracking code. G4beamline was specifically designed to carry out a full GEANT4 simulation of the MICE beam. It uses the full GEANT4 physics functionality to provide a more realistic simulation of the beamline, including all material descriptions and accurate magnetic field models for all magnets. The inclusion of large amounts of material in the beamline and the requirements of the phase space delivery of the beamline requires this more accurate simulation. The results from simulations using the tracking code will be shown in this chapter.

\subsubsection{Calibration of optical code materials to GEANT4 material description}

In order to set the beamline optics to supply a beam as close as possible to the nominal $\mathrm{p}=200 \mathrm{MeV} / \mathrm{c}, \varepsilon_{n}=6 \pi \mathrm{mm} \mathrm{mrad}$ setting, it is important to take into account the energy loss and multiple scattering due to materials placed in the way of the beam. In the MICE beamline, a large number of such materials exist - including vacuum windows, air, the PID detectors and the Diffuser to name but a few. As G4Beamline is used to make the final beam evaluation, a set of materials reference data was obtained from this code, as well as local beamline momenta, which could then be used to guide the material modelling in TRANSPORT and TURTLE. The materials were then implemented in TRANSPORT and TURTLE in preparation for a new beamline optics solution. 
Previous beamline tunes implemented in G4Beamline were carried out firstly by obtaining the optics solution designed using TRANSPORT/TURTLE. The associated currents were implemented in the G4Beamline magnetic elements and tweaked such that a reference particle traversed the channel. A disadvantage of this method is that the reference particle is tracked with all stochastic processes turned off. This includes multiple scattering. The only processes that are active are transportation which include electromagnetic fields, of course, and ionization energy loss with straggling disabled. Other disadvantages with the tune are that energy losses in the air, TOF0, Cherenkov1, TOF1 and the tracker windows were not taken into account. For the beamline tune carried out in September 2004 (SEPT04) this summed to a total momentum loss of $24 \mathrm{MeV} / \mathrm{c}$. This resulted in the momentum of the reference particle entering the Tracker1 vacuum being $183 \mathrm{MeV} / \mathrm{c}$ instead of the design value of $207 \mathrm{MeV} / \mathrm{c}$.

In order to strive at supplying a reference muon momentum of $200 \mathrm{MeV} / \mathrm{c}$, the approach involved working backwards from a $200 \mathrm{MeV} / \mathrm{c}$ muon at the centre of Absorber 2. As one moved upstream, crossing each material of interest, the local incoming momentum of the beam was determined by an iteration, until the outgoing momentum was that of the incoming momentum of the preceding element. Before each material, a probe beam was then generated at the correct incoming momentum, and the contribution to multiple scattering was calculated from

$$
\sqrt{\theta^{2}}=\sqrt{x_{r m s(\text { out })}^{\prime 2}-x_{r m s(i n)}^{\prime 2}}
$$

The probe beam used has the properties of being Gaussian with $\sigma_{X}=16.6 \mathrm{~mm}, \sigma_{Y}=16.6 \mathrm{~mm}$, $\sigma_{X^{\prime}}=0.00826$ radians, $\sigma_{Y^{\prime}}=0.00826$ radians with the mean momentum being varied to achieve the correct output momentum. Each beam consisted of $10 \mathrm{k} \mu^{+}$for each element downstream from and including the downstream decay solenoid window and $10 \mathrm{k} \pi^{+}$upstream from this. The results from this study can be seen in Table 7.4.

The consequence of this work has led to an alteration of the baseline pion capture momentum for the MICE beamline. To improve the TURTLE description of material effects in the beamline these results were used as a benchmark to which the TURTLE parameters were fitted. The choice was made to alter the density of the material regions within the TURTLE simulation, such that the momentum drop in each material was identical to that obtained using GEANT4 given the same input beam conditions. Generally this led to a reduction in density. 


\begin{tabular}{|c|c|c|c|c|c|c|}
\hline Beamline Element & $\begin{array}{l}\text { Pre- } \\
\text { element } \\
\text { mo- } \\
\text { mentum } \\
(\mathrm{MeV} / \mathrm{c})\end{array}$ & $\begin{array}{l}\text { Pre- } \\
\text { element } \\
x^{\prime}(\mathrm{rad})\end{array}$ & $\begin{array}{l}\text { Post- } \\
\text { element } \\
\text { Mo- } \\
\text { mentum } \\
(\mathrm{MeV} / \mathrm{c}) \\
\end{array}$ & $\begin{array}{l}\text { Post- } \\
\text { element } \\
x^{\prime}(\mathrm{rad})\end{array}$ & $\begin{array}{l}\Delta \mathrm{P} \\
(\mathrm{MeV} / \mathrm{c})\end{array}$ & $\theta$ (rads) \\
\hline Tracker Planes x2 & 208.05 & 0.00826 & 207.31 & 0.01019 & 0.74 & 0.005967 \\
\hline Tracker Window & 208.60 & 0.00826 & 208.08 & 0.0113 & 0.516 & 0.007711 \\
\hline Air $\quad$ (Diffuser $\rightarrow$ & 208.65 & 0.00826 & 208.63 & 0.008231 & 0.016 & 0.00714 \\
\hline Tracker Window & & & & & & \\
\hline Diffuser & 219.90 & 0.00826 & 208.62 & 0.08707 & 11.28 & 0.086677 \\
\hline Air $($ TOF $1 \rightarrow$ Diffuser & 220.00 & 0.00826 & 219.85 & 0.0086 & 0.15 & 0.002592 \\
\hline TOF1 & 232.15 & 0.00826 & 220.00 & 0.0261 & 12.15 & 0.024758 \\
\hline $\begin{array}{ll}\text { Air } & (\mathrm{CKOV} 1 \rightarrow \\
\text { TOF1 }) & \end{array}$ & 233.80 & 0.00826 & 232.13 & 0.0111 & 1.67 & 0.007481 \\
\hline CKOV1 & 241.80 & 0.00826 & 233.82 & 0.025 & 7.98 & 0.023596 \\
\hline $\begin{array}{ll}\text { Air } & (\mathrm{TOF} 0 \rightarrow \\
\text { CKOV1 }) & \end{array}$ & 242.26 & 0.00826 & 241.82 & 0.0091 & 0.44 & 0.003946 \\
\hline TOF0 & 253.90 & 0.00826 & 242.26 & $0 . .02333$ & 11.64 & 0.021787 \\
\hline $\begin{array}{l}\text { Air } \quad \text { (Proton } \\
\text { Absorber } \rightarrow \text { TOF0) }\end{array}$ & 255.48 & 0.00826 & 253.95 & 0.0106 & 1.53 & 0.006717 \\
\hline Proton Absorber & 265.90 & 0.00826 & 255.49 & 0.0204 & 10.41 & 0.018653 \\
\hline $\begin{array}{l}\text { Air (Decay Sol. Vac- } \\
\text { uum window } \rightarrow \text { Pro- } \\
\text { ton Absorber }\end{array}$ & 266.00 & 0.00826 & 265.88 & 0.084 & 0.12 & 0.001822 \\
\hline $\begin{array}{l}\text { Downstream decay } \\
\text { solenoid window }\end{array}$ & 266.20 & 0.00826 & 265.96 & 0.0091 & 0.24 & 0.003819 \\
\hline \multicolumn{7}{|l|}{$\begin{array}{l}\text { Individual CKOV1 el- } \\
\text { ements }\end{array}$} \\
\hline Front & 243.8 & 0.00826 & 243.32 & 0.0106 & 0.48 & 0.006643 \\
\hline CKOV & 243.8 & 0.00826 & 237.27 & 0.0226 & 6.53 & 0.021036 \\
\hline Window & 243.8 & 0.00826 & 242.96 & 0.011 & 0.84 & 0.007264 \\
\hline Light & 243.8 & 0.00826 & 243.68 & 0.0084 & 0.12 & 0.001527 \\
\hline \multicolumn{7}{|l|}{ Pion Channel } \\
\hline $\begin{array}{l}\text { Upstream decay } \\
\text { solenoid window }\end{array}$ & 440 & 0.00826 & 439.8 & 0.0086 & 0.2 & 0.002394 \\
\hline $\begin{array}{l}\text { Beamline vacuum } \\
\text { window }\end{array}$ & 440.2 & 0.00826 & 440 & 0.089 & 0.2 & 0.003314 \\
\hline $\begin{array}{l}\text { Air (ISIS vacuum } \rightarrow \\
\text { beam vacuum ) }\end{array}$ & 440.23 & 0.00826 & 440.21 & 0.0083 & 0.02 & 0.000814 \\
\hline ISIS vacuum window & 440.43 & 0.00826 & 440.2 & 0.009 & 0.23 & 0.003574 \\
\hline
\end{tabular}

Table 7.4: Summary of study of momentum drop and multiple scattering analysis in the MICE beamline. 


\subsection{G4Beamline simulation}

\subsubsection{Implementation of magnetic elements}

The implementation of magnetic fields in G4beamline is of utmost importance. The realism of the simulation and hence accuracy of the design relies heavily on the magnetic fields calculated by the program. The placement of a field is naturally associated with a physical volume. Hence the placing of a magnet involves both the placing of the field and material.

The description of both dipoles involves placing a rectangular cuboid of width $=1828 \mathrm{~mm} \times$ height $=1320 \mathrm{~mm} \times$ length $=990 \mathrm{~mm}$. Placed with this element is the extended field region which has the dimensions: width $=660 \mathrm{~mm} \times$ height $=200 \mathrm{~mm} \times$ length $=1038 \mathrm{~mm}$. The aperture for the pion capture dipole is in vacuum, where as the aperture for the second dipole is air. In the simulation one tunes this bend by specifying the $B_{y}$ component of the dipole field. For ease of placement the centreline of the simulation is manually rotated by 60 degrees and 30 degrees for the first and second magnet respectively. The centerline is the axis where any physical volume (except the world volume) is placed in G4Beamline. One then ensures that the $x$ and $x^{\prime}$ values for a reference particle tracked far downstream traces the centerline. This requires the disabling of all stochastic processes in the program.

In the first stage of the design of a new beamline optics tune the beamline is evaluated by TRANSPORT and TURTLE. This code allows for fast iteration by enabling quick transport of the beam via matrix manipulation, but this has the inherent limitations provided by these methods. In both these beam optics codes, fringe fields are modelled using the thin lens approximation. To further improve the realism of the implementation of the dipoles the program has been extended to include field maps for these magnets. The maps are produced using TOSCA [102] and then implemented in the code. The advantage of this is that the complete field of the actual magnet is calculated including non-approximate fringe fields.

The beamline contains two different types of quadrupole magnets. The Type IV quads are modelled as iron volumes with an inner aperture radius of $101.5 \mathrm{~mm}$ and outer magnet radius of $381 \mathrm{~mm}$ with a longitudinal extent along the beam direction of $914 \mathrm{~mm}$. The effective field length placed with this volume extends to a length along the z-axis of $853.4 \mathrm{~mm}$. The quadrupole strength is dependent on the tune and is expressed as a field gradient providing vertical or 
horizontal focusing. Apertures are vacuum filled.

The Q35 matching quadrupoles are larger with "rounded cross" apertures. These are constructed in the software as iron volumes $1046 \mathrm{~mm}$ long with an outer radius of $700 \mathrm{~mm}$. The apertures are modelled using circles for the pole tips with the radius of the inside of the coil, the inner radius of the pole tips defined by the user. The aperture is filled with air as these are placed downstream with no vacuum provision. It should be noted that the outside geometry of these quadrupoles is not accurate and the magnet mounts are not implemented. Again this does not affect the realism of the beam. The Q35 magnets also have mirror plates attached. The mirror plates [103] are effectively small additional coils that provide an equal but opposite field to the fringing field of the quadrupole. These effectively terminate the field at the effective field boundary of the magnet.

The placement of the decay solenoid is a cylindrical volume of iron $5000 \mathrm{~mm}$ long with an inner bore of $97 \mathrm{~mm}$ and outer radius of $180 \mathrm{~mm}$. A field map was calculated using TOSCA [102] for a $3.7 \mathrm{~T}$ field and this is placed with the volume and a conducting copper coil with a user defined current. This map is used for each tune of the beamline with the map scaled to the design field by modification of the coil current. To increase tracking efficiency a shield of dimensions: inner radius $=180 \mathrm{~mm}$; outer radius $=1000 \mathrm{~mm}$ is placed around the upstream end of the solenoid. This acts to kill completely the tracking of any particles which do not fall into the solenoid acceptance. One further iron volume, inner radius $=48 \mathrm{~mm}$, outer radius $=180 \mathrm{~mm}$, length $=68 \mathrm{~mm}$ is implemented at the upstream face of the solenoid to mimic the reduced aperture of the solenoid at this point.

It should be noted that a simplified version of the MICE experiment is also implemented in G4Beamline. This can be useful to measure the rates of muons deemed to pass through the whole MICE experiment. It is mentioned here as it is important to include the tracker1 field in the beamline simulation although it is strictly not part of the beamline as the fringe field can extend into the upstream end of the beamline. This solenoid is represented in a similar manner to the decay solenoid, again using a TOSCA field map 


\subsubsection{Implementation of beamline detectors}

Generally in beamline design studies the detectors are modelled simplistically. The important factors are: accurate modelling of the transverse size of each element to ensure that the detectors are spatially large enough to adequately cover the beam dimensions; correct modelling of the axial thickness of the detectors such that the effects of multiple scattering and momentum losses can be simulated; accurate modelling of the positions of the elements along the beamline such that the effect of the lattice on the beam at the vicinity of the elements can be simulated. Provision is not made to include any detector responses, no digitisation or reconstruction is performed so the studies in this section are made using Monte Carlo truth information. The detailed implementation of detector response are deemed outside the remit of the beamline design team and are more thoroughly modelled using the full GEANT4 MICE detector implementation (G4MICE framework) [76].

The general concept when constructing the detector/material geometry is only to include the active material in each detector and any detector materials which the beam will pass through. Hence detector supports, PMT housings etc. are not modelled. This is an adequate assumption as this material is distant enough from the beam axis that very few particles will be incident and even if some outliers do interact they will fall out of the phase-space transported by the lattice. A summary of the volume construction can be seen in Tables 7.5, 7.6. The time-offlight detectors are modelled simply as a sheet of Bicron BC-404 scintillator with the specified dimensions. This description is adequate for design purposes as the important characteristics are the beam-spot size and singles rate in the vicinity of the detector locations. These can be sampled by making the detector volume a GEANT active volume so that MC truth data can be extracted. The implementation of the Cherenkov device is more complicated. The CKOV that has been included in the simulation that will be shown is the original single unit detector. The representation (see Figure 7.6) includes an upstream aluminium window, fluorocarbon radiator, quartz window and air light guides.

Modelling these detectors in the way described drives certain aspects of detector design. The momentum distributions such as those shown in Figure 7.8 are important when considering densities for aerogel detectors. It is also important to evaluate that the beamline can provide sufficient $\pi / \mu$ separation. As such, time of flight plots such as those in Figure 7.8 can be used 


\begin{tabular}{|c|c|c|c|c|}
\hline Element & Material & Dimensions $(\mathrm{mm})$ & Position $(\mathrm{mm})$ & $\# X_{0}$ \\
\hline \hline TOF0 & Polyvinyltoluene & Cuboid: $(\mathrm{x}, \mathrm{y}, \mathrm{z})=500,500,50.8$ & $\mathrm{z}=20811.6$ & 0.12 \\
CKOV1 & Detailed CKOV implementation in Table 7.6 & $\mathrm{z}=21062.4$ & \\
TOF1 & Polyvinyltoluene & Cuboid: $(\mathrm{x}, \mathrm{y}, \mathrm{z})=480,480,50.8$ & $\mathrm{z}=28894.5$ & 0.12 \\
\hline
\end{tabular}

Table 7.5: Summary of material content of beamline introduced by particle identification detectors.

\begin{tabular}{|c|c|c|c|}
\hline CKOV component & Material & Dimensions $(\mathrm{X}, \mathrm{Y}, \mathrm{Z})(\mathrm{mm})$ & $\# X_{0}$ \\
\hline \hline Front & Aluminium & $400 \times 400 \times 1$ & 0.01 \\
Radiator & $C_{6} F_{14}, \rho=1.7 \mathrm{gcm}^{-3}$ & $400 \times 400 \times 20$ & 0.10 \\
Window & Quartz & $400 \times 400 \times 2$ & 0.02 \\
Light & Air & $400 \times 400 \times 478$ & 0.002 \\
\hline
\end{tabular}

Table 7.6: Original single unit CKOV implementation.

in conjunction with MC truth data sampled at the position of the tracker to provide very useful information on separating the distributions as shown in Figure 7.9. To construct these plots the time-of-flight between both beamline TOF walls were compared to the Monte Carlo truth momentum sampled at the tracker. To simulate the effect of the TOF wall timing resolution both TOF time stamps were smeared with a 70ps Gaussian distribution.

\subsubsection{Implementation of non-detector materials in beamline}

The baseline beamline contains significant volumes of inactive material which are summarised in Table 7.7. Upstream in the pion channel there are four vacuum windows through which the beam must pass. One which terminates the ISIS vacuum, one at the upstream end of the beamline vacuum and one attached to each end of the PSI decay solenoid. These vacuum windows are modelled as $120 \mathrm{~mm}$ radius, $0.5 \mathrm{~mm}$ thick aluminium cylinders and affect the beam very little. The drift spaces are filled with vacuum from the target to the downstream end of the decay solenoid, with the exception of a $99 \mathrm{~mm}$ section of air to reflect the ISIS/Beamline vacuum separation. The entire muon matching section including the Type Q35 quad apertures are open to the hall atmosphere and as such this section is modelled as air at standard temperature and pressure (STP).

To filter out the high proton content of the beam downstream from the decay solenoid the simulation includes a thick segment of polyethylene with dimensions: width $=400 \mathrm{~mm}$; 

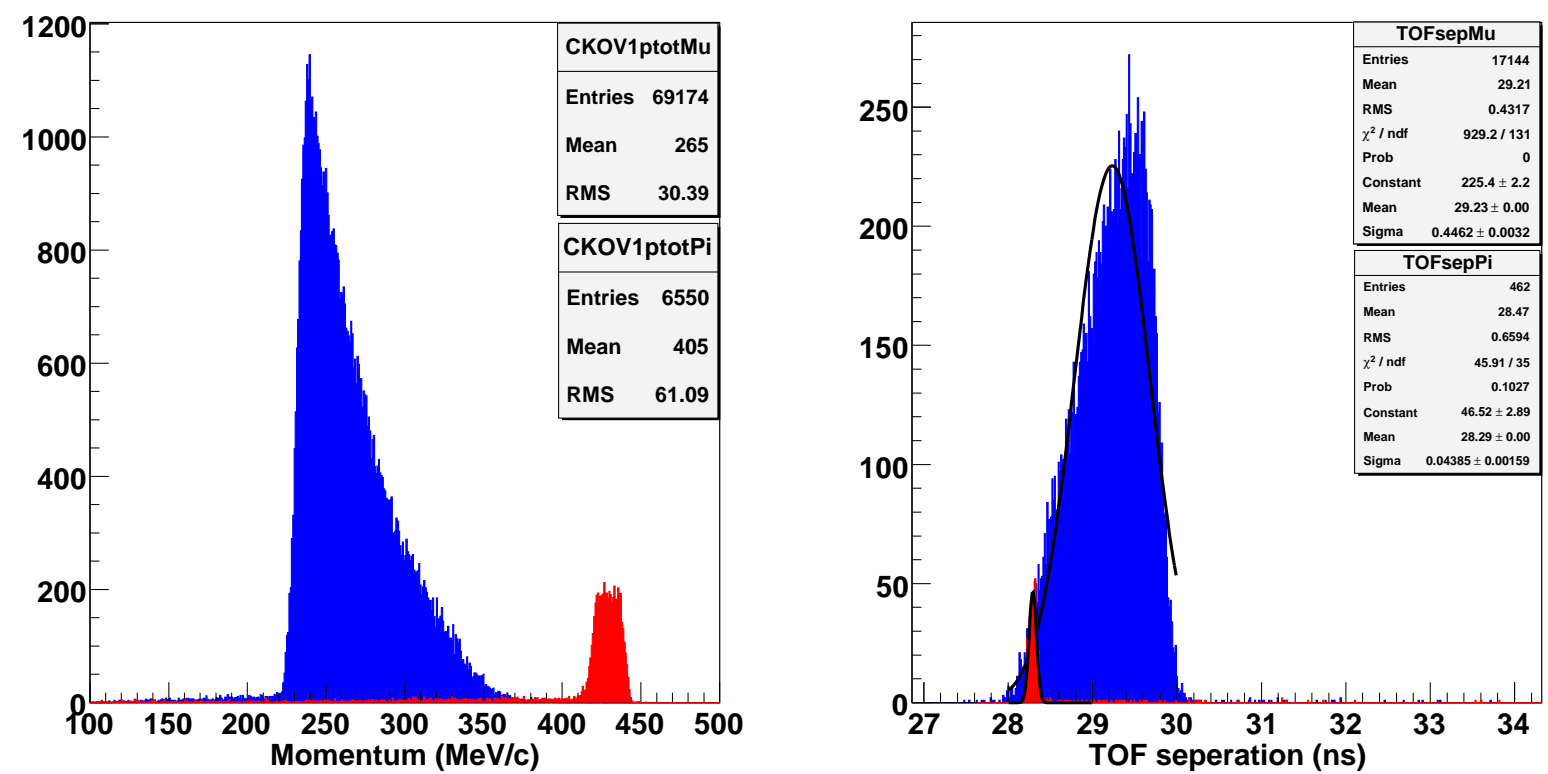

Figure 7.8: Analysis of momentum distribution for pions (red) and muons (blue) sampled entering the upstream Cherenkov detector (left panel). Typical time-of-flight separation between pions (red) and muons (blue) (right panel). Results obtained from CM14-6 $-200 \mathrm{MeV}$-Walaron tune.
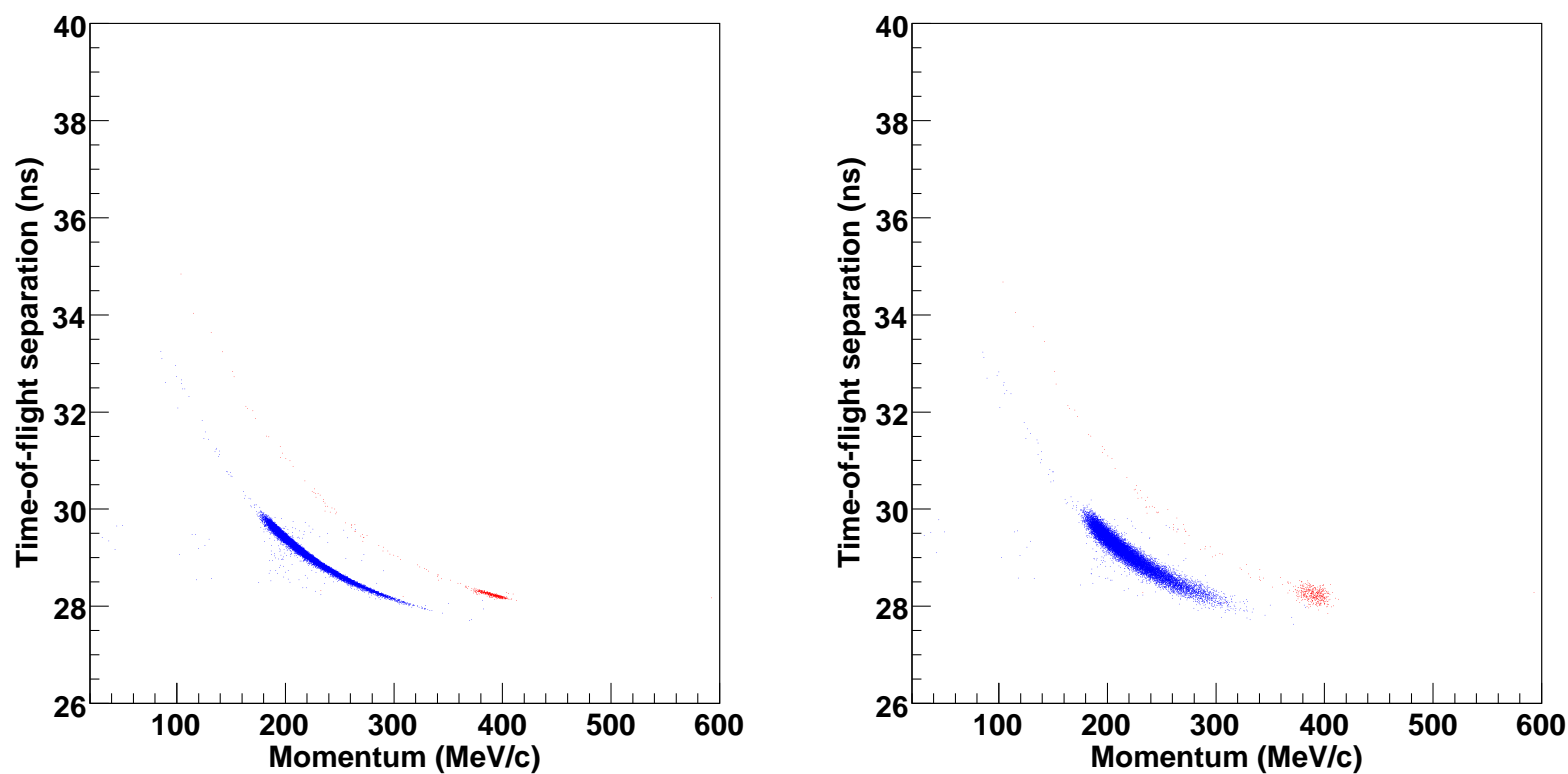

Figure 7.9: Analysis of idealised particle identification of pions (red) and muons (blue), achievable by the MICE beamline (left panel). Particle identification achievable by the beamline with estimation of the effect of TOF wall timing resolution (right panel). Results obtained from CM14-6 $\pi-200 \mathrm{MeV}$-Walaron tune 


\begin{tabular}{|c|c|c|c|c|}
\hline Element & Material & Dimensions $(\mathrm{mm})$ & $\mathrm{z}(\mathrm{mm})$ & $\# X_{0}$ \\
\hline \hline Proton Filter & Polyethylene & Cuboid: $x=400, y=400, z=50$ & 15045.9 & 0.17 \\
Iron shield & Iron & Radii(inner=250, outer $=750), \mathrm{z}=100$ & 28969.1 & 0.57 \\
Diffuser & Lead & Radius $=200$, variable thickness & 29470 & 0.17 \\
\hline
\end{tabular}

Table 7.7: Summary of non-detector material content of beamline, for radiation length calculation the diffuser for the $7.1 \pi \mathrm{mm}$ mrad beamline is assumed

height $=400 \mathrm{~mm}$; length $=50 \mathrm{~mm}$. The presence of this material at $\mathrm{z}=15070.9$ (center of volume) reduces the proton content of the beam dramatically. The largest impact of material on the beamline happens further downstream caused by the lead beam diffuser. The lead diffuser is modelled as a lead disk of radius $20 \mathrm{~cm}$ with the thickness determined by the beamline tune. By design, this device drastically increases the divergence of the beam and marks the end of the MICE beamline.

\subsection{Analysis of a $200 \mathrm{MeV} / \mathrm{c}$ muon beamline for the MICE experiment}

This section will discuss the results for a $200 \mathrm{MeV} / \mathrm{c}$ beamline tune for three different emittance cases. The $200 \mathrm{MeV} / \mathrm{c}$ case is the most important as it is the nominal case for MICE studies. The results are obtained from tracking $\pi^{+}$from the target location along an accurate representation of the beamline in the GEANT4 based G4beamline program. Analysis is performed using a combination of programs Histoscope [55], G4MICE [76] and ROOT [66] all of which have been previously referenced. The important features in terms of analysis of the beamline are to check consistency of transverse beam widths with detector sizes, measure beam purity along beamline and finally analyse the momentum distribution and emittance achieved at the upstream tracker. For reference, the optics solutions used to obtain the analysed beams can be seen in Table 7.8. It should be noted that the optics tune for this beamline in TURTLE was for the single CKOV device and as such the analysis was carried with this device modelled for consistency. The inclusion of the new device has been studied and does alter the results obtained slightly if one uses the optimisation for the old device (as expected), slight modifications to the optics in TRANSPORT are required but this will not be a problem. The results presented are still an 
accurate representation.

\begin{tabular}{|c|c|c|c|c|}
\hline Beamline element & $1 \pi$ & $7.1 \pi$ & $10 \pi$ & Centerline coordinate $(\mathrm{mm})$ \\
\hline \hline Quad:Type 4 (Q1) & 1.1110 & 1.1673 & 1.1895 & 2573.3 \\
Quad:Type 4 (Q2) & -1.3887 & -1.4591 & -1.4869 & 3973.7 \\
Quad:Type 4 (Q3) & 0.9668 & 1.0157 & 1.0351 & 5373.3 \\
Dipole:Type1 NIM (B1) & 1.3589 & 1.4277 & 1.4549 & 7434.2 \\
Solenoid: PSI decay & 4.0098 & 4.2131 & 4.2934 & 9664.7 \\
Dipole:Type1 NIM (B2) & 0.4056 & 0.4293 & 0.4387 & 15281.7 \\
Quad:Q35 (Q4) & 0.9347 & 0.9897 & 1.0113 & 17331.6 \\
Quad:Q35 (Q5) & -1.2520 & -1.3256 & -1.3547 & 18491.6 \\
Quad:Q35 (Q6) & 0.8307 & 0.8876 & 0.7852 & 19651.6 \\
Quad:Q35 (Q7) & 0.8635 & 0.8876 & 0.7852 & 24963.7 \\
Quad:Q35 (Q8) & -1.2668 & -1.3427 & -1.2418 & 26123.7 \\
Quad:Q35 (Q9) & 1.0992 & 1.1475 & 1.0110 & 27283.7 \\
\hline
\end{tabular}

Table 7.8: Beamline optics solutions for $200 \mathrm{MeV} / \mathrm{c}$ MICE beamline. Dipole and solenoidal fields expressed in $\mathrm{T}$, quadrupole fields given in $\mathrm{Tm}^{-1}$

\subsubsection{Mid-emittance $200 \mathrm{MeV} / \mathrm{c}$ tune}

The evaluation of the mid-emittance tune of the MICE beamline is presented here. The initial design as implemented in TURTLE aimed at producing a $7.1 \pi \mathrm{mm}$ mrad beam at the tracker.

As discussed, GEANT4 will be the final beamline study tool. Therefore the geometry of the GEANT4 simulation was implemented including realistic magnetic fields and materials. The correct optics solutions for this tune can be seen in Table 7.8. The diffuser thickness included in this tune is $9.6 \mathrm{~mm}$. The simulation involved the tracking of an initially pure 1 million $\pi^{+}$beam from the MICE target.

The transverse particle distributions of this beam sampled at each of the beamline detector locations can be seen in Figure 7.10. It is important that the transverse beam width is compatible with the active transverse dimension of the detectors. The most important distribution is the "Good $\mu^{+}$". These are the muons which are successfully transported through the MICE experiment and record hits in all three time-of-flight detectors, both upstream and downstream trackers and finally the calorimeter. This will be the muon distribution for which cooling will be measured. It can be seen from Figure 7.10 that the full beam width at TOF0 is adequately covered by the detector but more importantly this means the Good $\mu^{+}$distribution falls easily 
inside the detector acceptance. This is also the case for the $y$-distribution of the beam at the Cherenkov. The total muon horizontal distribution is slightly larger than the detector acceptance. This is not a worry however as the Good $\mu^{+}$coverage is good. Again, at TOF1, the detector acceptance is comfortably large enough to accommodate the beam.

The purity of the beam that the beamline transports is important. This is achieved by careful choice of optics and the proton absorber. A summary of the particle content of the beam at each detector is shown in Table 7.9. One can see that the non-muon contamination of the beamline is very small with no protons present after TOF0, negligible positron contamination and a very small $\pi^{+}$population ranging from $\approx 1 \%$ at TOF0 to negligible at TOF1. Hence the beamline does provide an extremely pure $\mu^{+}$beam. It is relatively easy to produce a very pure beam if one chooses to neglect transmission, thus the beamline must also transport particles at a rate compatible with MICE. The analysis of beamline rates, normalised as discussed previously in this chapter, is presented in Table 7.10. This shows the rates sampled at each of the beamline detectors and the Good $\mu^{+}$rate. From this, one can see that the beamline easily transports enough positive muons to the MICE experiment with a mean Good $\mu^{+}$rate of $\approx 1 \mathrm{MHz}$. This is equivalent to approximately 1000 good muons in the $1 \mathrm{~ms}$ that the target dips into the beam each cycle.

\begin{tabular}{|c|c|c|c|c|}
\hline Particle & TOF0 (\%) & CKOV (\%) & TOF1 (\%) & Tracker (\%) \\
\hline \hline$\mu^{+}$ & 98.32 & 99.14 & 99.80 & 99.92 \\
$\pi^{+}$ & 0.99 & 0.82 & 0.08 & 0.08 \\
$e^{+}$ & 0.07 & 0.04 & 0.12 & 0 \\
Proton & 0.62 & 0 & 0 & 0 \\
\hline
\end{tabular}

Table 7.9: Purity of $6 \pi, 200 \mathrm{MeV} / \mathrm{c}$ beamline sampled at each of the beamline detectors and upstream tracker

\begin{tabular}{|c|c|c|c|c|}
\hline Downstream position & LAHET & GEANT4 & MARS & Mean rate \\
\hline \hline TOF0 & 3.17 & 2.72 & 3.54 & $3.15 \pm 0.14$ \\
CKOV1 & 3.07 & 2.64 & 3.43 & $3.05 \pm 0.13$ \\
TOF1 & 1.35 & 1.16 & 1.51 & $1.34 \pm 0.06$ \\
Tracker 1 & 1.34 & 1.16 & 1.50 & $1.33 \pm 0.06$ \\
Good $\mu^{+}$ & 1.08 & 0.93 & 1.21 & $1.08 \pm 0.05$ \\
\hline
\end{tabular}

Table 7.10: Positive muon rates in $\mathrm{MHz}$ along the mid-emittance $200 \mathrm{MeV} / \mathrm{c}$ MICE beamline 

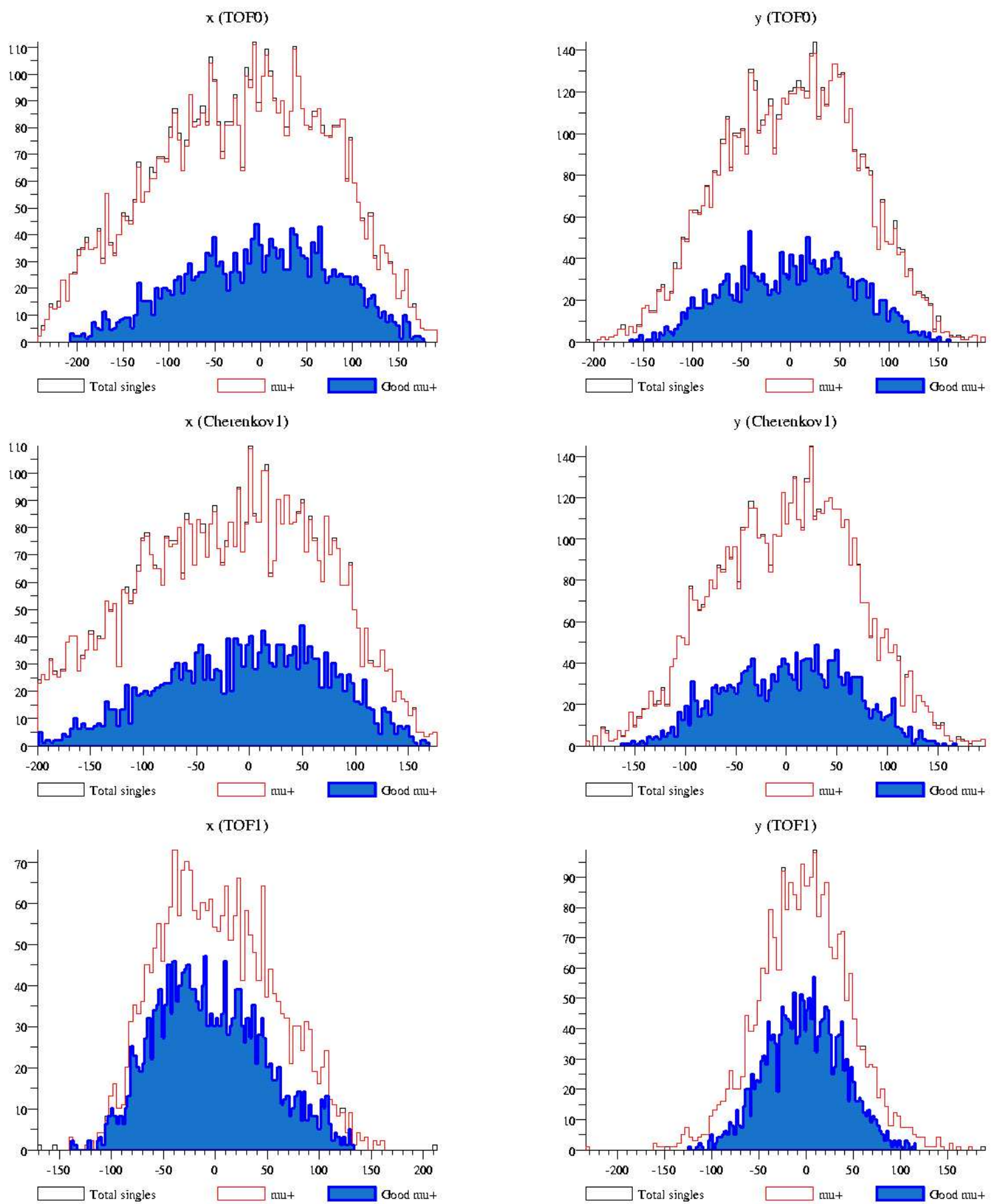

Figure 7.10: Results of GEANT4 implementation of the CM14- $6 \pi-200 \mathrm{MeV} / \mathrm{c}$ beamline optics tune. The plots show the $\mathrm{X}$ and $\mathrm{Y}(\mathrm{mm})$ spatial distributions of the beam at Time-of-flight detectors 0 and 1 and at the Cherenkov detectors. The dimensions of these detectors are TOF0 $\pm 250 \mathrm{~mm}$, TOF $1 \pm 240 \mathrm{~mm}$ and $\mathrm{CKOV} \pm 200 \mathrm{~mm}$.

It has been shown that the beam is compatible with current beamline detectors and is very pure. A further constraint of the beamline is that the momentum distribution delivered by the beam inside the tracker should be achieved. This baseline value of $200 \mathrm{MeV} / \mathrm{c}$ is designed to be 
the momentum of the beam at the centre of the first hydrogen absorber. This is placed downstream close to the tracker. The corresponding momenta desired in the tracker is consequently peaked at $207 \mathrm{MeV} / \mathrm{c}$ to take account of the momentum drop in the tracker planes, absorber window and hydrogen. Figure 7.11 shows the momentum distribution sampled at the tracker. It can be seen from this plot that the $\mu^{+}$distribution is indeed peaked at the correct momentum of 202-210 MeV/c. It can also be seen that the Good $\mu^{+}$phase-space is shifted to lower momenta, this is due to interaction with materials in the MICE channel.

Ptot(Upstrean tracker)

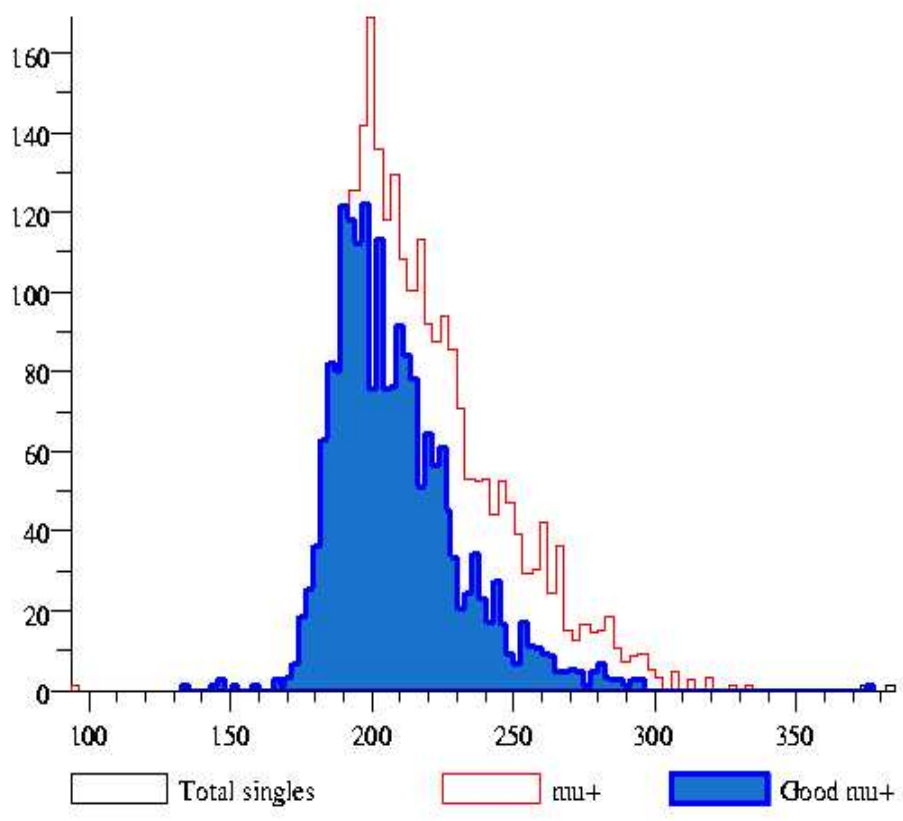

Figure 7.11: Ptot distribution at the entrance to the upstream MICE tracker for the CM14$6 \mathrm{pi}-200 \mathrm{MeV} / \mathrm{c}$ tune

Finally, the evolution of the phase-space that the beamline is designed to transport is evaluated. The initial TRANSPORT design aims to transport a beam such that emittance of the beam sampled at the upstream tracker within a $\pm 10 \%$ momentum spread was $7.1 \pi \mathrm{mm}$ mrad. Figure 7.12 shows the envolution of the $\sigma_{x}$ and $\sigma_{y}$ distributions for those particles which have a momentum of $207 \pm 10 \%$ measured at the upstream tracker. Unfortunately it is not possible to place sampling planes within magnetic elements using the tracking program and as such the envelopes within the quadrupole magnets are not calculated. Also shown is an accurate representation of the downstream muon matching section. The transverse size of all magnets, detectors and materials are accurate as are the longitudinal dimension of all quadrupole magnets, 
detectors and materials. The final two plots in Figure 7.12 show the evolution of momentum and emittance for the same phase-space. Again, in these plots we have a representation of beamline elements which is correct longitudinally. It can be seen from this that the transport of the design beam easily passes through the channel apertures without any scraping and one can see how the longitudinal momentum component is modified through the matching section. The emittance transported to the tracker by this beamline is $8.9 \pi \mathrm{mm} \mathrm{mrad}$ which does differ from the emittance achieved by TRANSPORT/TURTLE, as expected. GEANT4 is a much more accurate simulation engine compared with optics codes. The key difference attributed to this is in the way the beam interaction in materials is modelled. It has been discussed that the material description in the optics codes were modified to produce the correct momentum decrease across the volume. This in turn leads to an underestimate of the multiple scattering in the material of approximately $20 \%$. Again, the basic design from the optics code is used to steer the GEANT4 simulation and, while the $7.1 \pi \mathrm{mm}$ mrad value is the value of the emittance that we were aiming for, it is not crutial since we will run with a number of different beam emittance values. Hence even though there is a difference, this beamline still provides a good mid-emittance beam into the MICE experiment.

\subsubsection{Large emittance $200 \mathrm{MeV} / \mathrm{c}$ tune}

This section presents the analysis of a beamline designed to provide a large emittance beam. The analysis follows the same pattern as the analysis presented in the previous section. The initial design in TRANSPORT achieved a $10 \pi \mathrm{mm}$ mrad emittance in the tracker. The differences in this beamline compared with the mid emittance beamline is that it has a different optics tune, as summarised in Table 7.8 and a thicker lead beam degrader of $13.3 \mathrm{~mm}$. It can be seen from the transverse beam profiles shown in Figure 7.13 that the detector active areas are large enough that the Good $\mu^{+}$distributions can be sampled at all detector locations.

The purity of the $\mu^{+}$beam is high with contamination much lower than $1 \%$ for the beam entering the first tracker (see Table 7.11). The rates achievable with this optics solution easily accomplish the MICE baseline rate with normalisation details again given in Table 7.12. The momentum distribution at the upstream tracking detector is correct (see Figure 7.14), as was the case in the mid-emittance tune. From the information given it can be seen that again this 

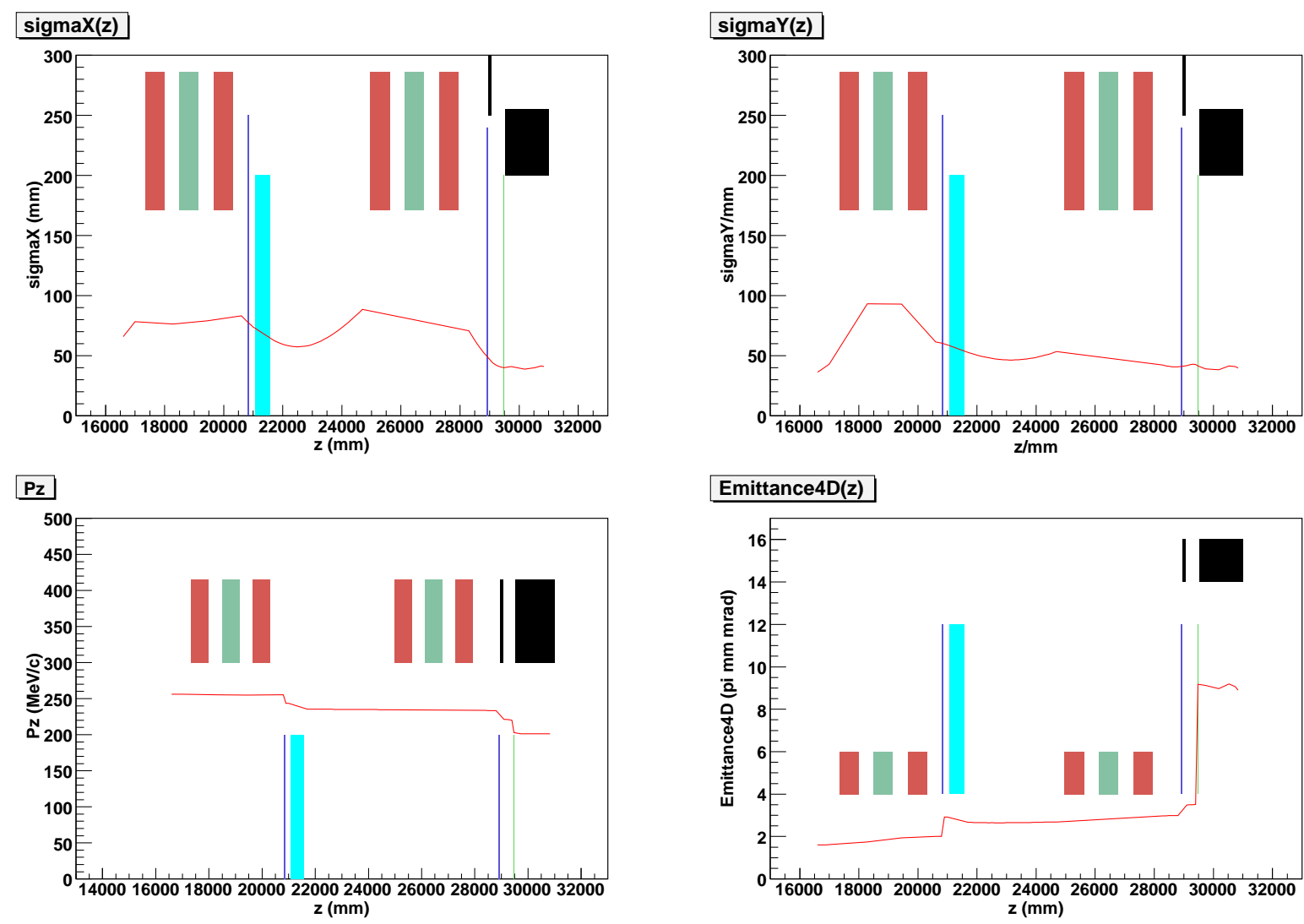

Figure 7.12: Results of GEANT4 implementation of the CM14- $6 \pi-200 \mathrm{MeV} / \mathrm{c}$ beamline optics tune. Plots show the evolution of the beam properties over the muon matching section. These properties are calculated for the reduced phase space volume with $215.31<E_{\mu}<252.2$ sampled at the first MICE tracker. Schematic representation of the beamline is indicated by different volumes on plots according to the following key: Matt red (horizontal focussing quad); Matt green (horizontal defocussing quad); Thin black (Iron shield); Thick black (tracker solenoid, only aperture correct); Blue (TOF0 and TOF1); Thick light blue (CKOV); Thin green (Diffuser)

beamline succesfully achieves the required purity, rates and momentum. The emittace of the beam transported as calculated in the upstream tracker can be seen in Figure 7.15. These results exhibit the same trend as the previously analysed tune i.e. the emittance obtained from the tracking code is larger than that achieved using matrix manipulation with G4beamline achieving an emittance of $\approx 12.3 \pi \mathrm{mm}$ mrad. This has been attributed to the differences in how both codes model the effect of multiple coloumb scattering in the beamline materials.

Even with this difference it is correct to conclude that this beamline does achieve its goal of transporting a high purity, $207 \mathrm{MeV} / \mathrm{c} \mu^{+}$beam with an emittance at the top end of the emittance range for which the MICE experiment wishes to measure cooling. In addition, the beamline successfully transports this beam at a rate larger than the baseline considered for 

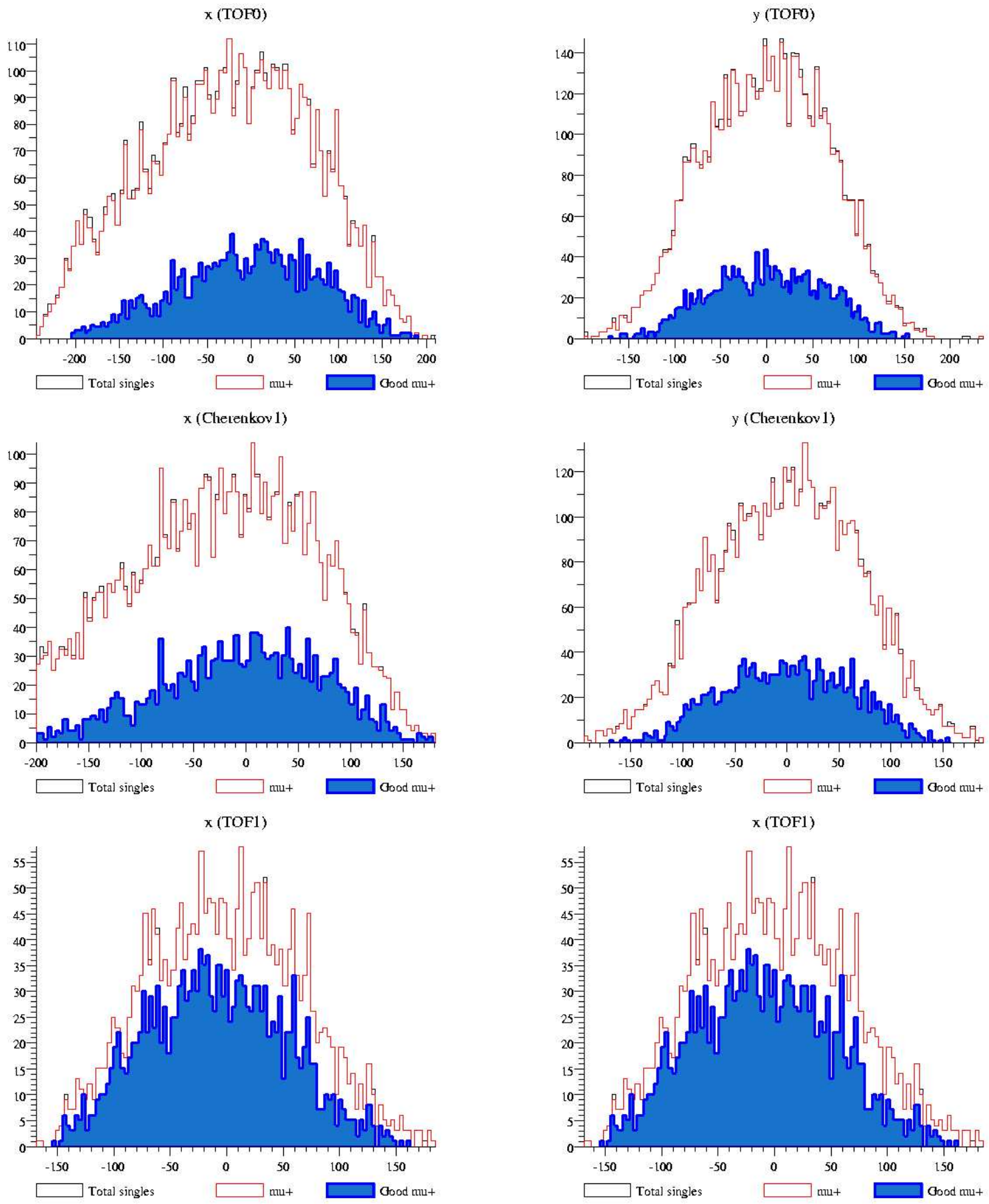

Figure 7.13: Results of GEANT4 implementation of the CM14-10 $\pi-200 \mathrm{MeV} / \mathrm{c}$ beamline optics tune. The plots show the $\mathrm{X}$ and $\mathrm{Y}(\mathrm{mm})$ spatial distributions of the beam at Time-of-flight detectors 0 and 1 and at the Cherenkov detectors. The dimensions of these detectors are TOF0 $\pm 250 \mathrm{~mm}$, TOF $1 \pm 240 \mathrm{~mm}$ and $\mathrm{CKOV} \pm 200 \mathrm{~mm}$.

MICE running. 


\begin{tabular}{|c|c|c|c|c|}
\hline Particle & TOF0 (\%) & CKOV (\%) & TOF1 (\%) & Tracker $(\%)$ \\
\hline \hline$\mu^{+}$ & 98.43 & 99.11 & 99.75 & 99.96 \\
$\pi^{+}$ & 0.92 & 0.70 & 0.12 & 0.04 \\
$e^{+}$ & 0.14 & 0.14 & 0.13 & 0 \\
Proton & 0.51 & 0.02 & 0 & 0 \\
\hline
\end{tabular}

Table 7.11: Purity of $10 \pi, 200 \mathrm{MeV} / \mathrm{c}$ beamline sampled at each of the beamline detectors and upstream tracker

\begin{tabular}{|c|c|c|c|c|}
\hline Downstream position & LAHET & GEANT4 & MARS & Mean rate \\
\hline \hline TOF0 & 2.95 & 3.48 & 2.43 & $2.96 \pm 0.18$ \\
CKOV1 & 2.85 & 3.37 & 2.35 & $2.86 \pm 0.17$ \\
TOF1 & 1.24 & 1.46 & 1.02 & $1.24 \pm 0.07$ \\
Tracker 1 & 1.23 & 1.45 & 1.01 & $1.23 \pm 0.07$ \\
Good $\mu^{+}$ & 0.84 & 1.02 & 0.71 & $0.87 \pm 0.05$ \\
\hline
\end{tabular}

Table 7.12: Positive muon rates in $\mathrm{MHz}$ along the large emittance $200 \mathrm{MeV} / \mathrm{c}$ MICE beamline

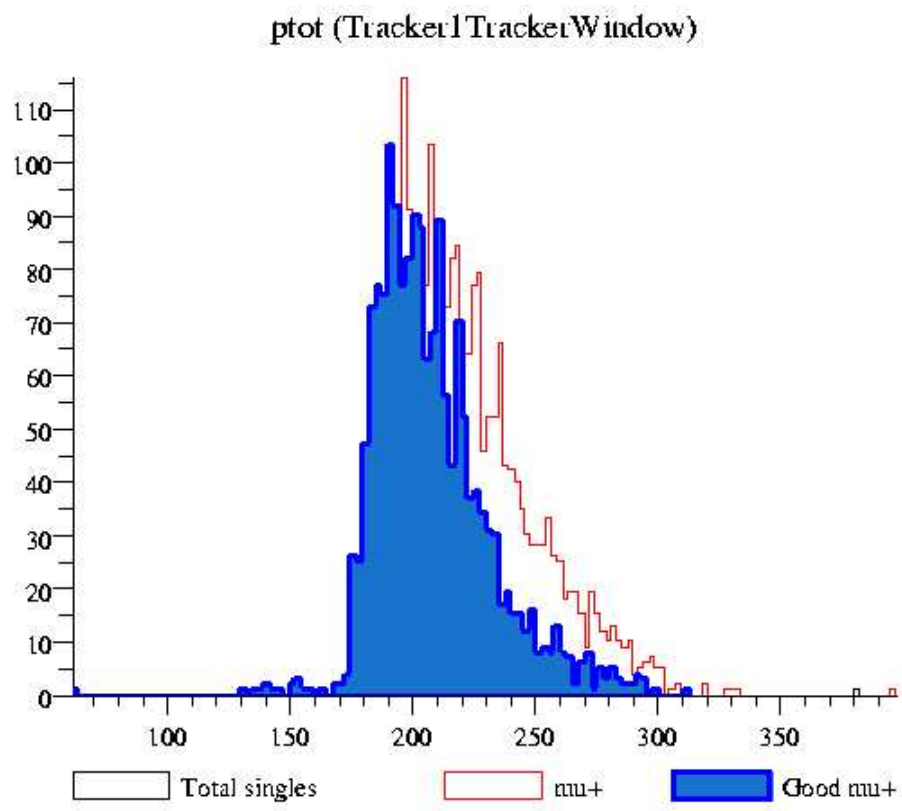

Figure 7.14: Ptot distribution at the entrance to the upstream MICE tracker for the CM14$10 \mathrm{pi}-200 \mathrm{MeV} / \mathrm{c}$ tune

\subsubsection{Low $\pi 200 \mathrm{MeV} / \mathrm{c}$}

This final analysis section presents the analysis of a beamline design aimed at providing a low emittance beam into the MICE experiment. The initial design using the optics codes provided a $1 \pi \mathrm{mm}$ mrad beam into the experiment. This tune involves unique magnet current solutions, 

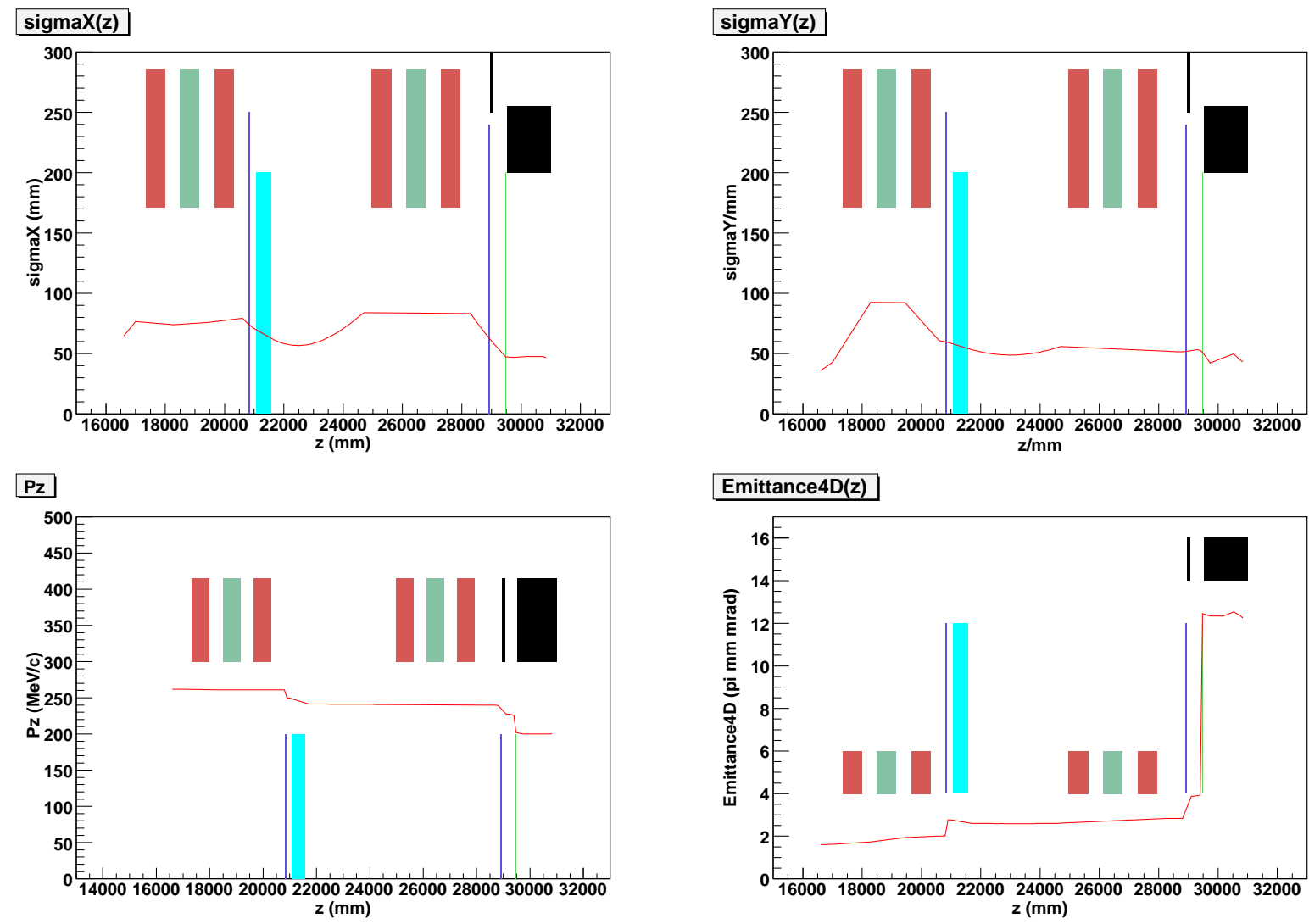

Figure 7.15: Results of GEANT4 implementation of the CM14-10 $\pi-200 \mathrm{MeV} / \mathrm{c}$ beamline optics tune. Plots show the evolution of the beam properties over the muon matching section. These properties are calculated for the reduced phase space volume with $215.31<E_{\mu}<252.2$ sampled at the first MICE tracker. Schematic representation of the beamline is indicated by different volumes on plots according to the following key: Matt red (horizontal focussing quad); Matt green (horizontal defocussing quad); Thin black (Iron shield); Thick black (tracker solenoid, only aperture correct); Blue (TOF0 and TOF1); Thick light blue (CKOV); Thin green (Diffuser)

the complete removal of the lead diffusers and downstream collimation.

It is not possible to achieve a beam of $1 \pi \mathrm{mm}$ mrad purely from manipulation of magnet currents. The beam has a natural emittance of approximately $3-4 \pi \mathrm{mm} \operatorname{mrad}$. This stems from irreducible multiple scattering induced emittance increases along the beamline. As this beamline must provide an emittance smaller than the natural emittance of the beamline there is no need to apply beam degradation with a diffuser. In addition, this design includes the placement of a $300 \mathrm{~mm}$ long cylindrical iron diffuser with an inner radius of $26 \mathrm{~mm}$ and outer radius of $300 \mathrm{~mm}$ placed between TOF1 and the upstream tracker. The implications of this feature on beamline performance, specifically rate, will be discussed later.

The histograms shown in Figure 7.16 confirm that the detectors are easily large enough to 

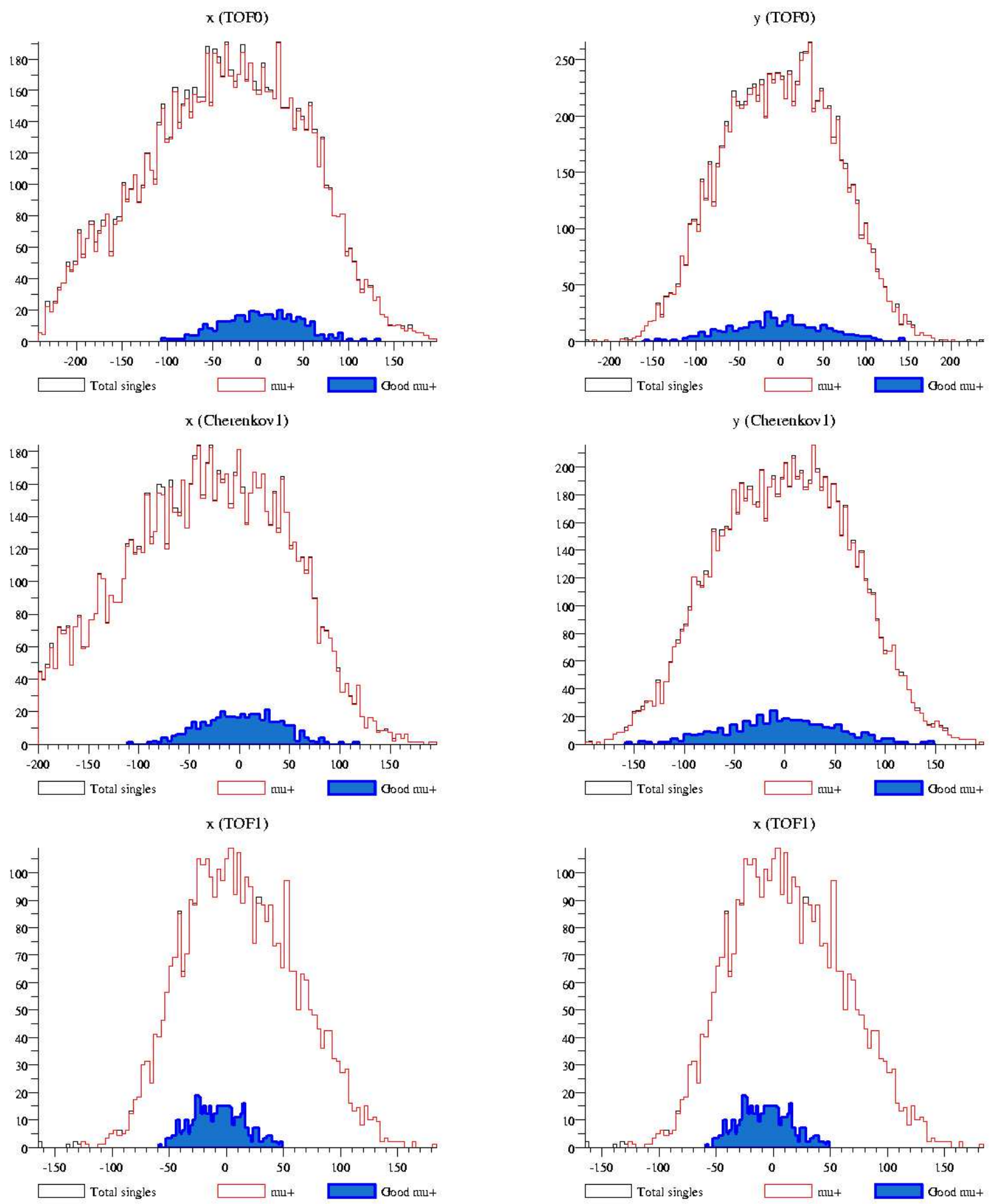

Figure 7.16: Results of GEANT4 implementation of the CM14- $1 \pi-200 \mathrm{MeV} / \mathrm{c}$ beamline optics tune. The plots show the $\mathrm{X}$ and $\mathrm{Y}(\mathrm{mm})$ spatial distributions of the beam at Time-of-flight detectors 0 and 1 and at the Cherenkov detectors. The dimensions of these detectors are TOF0 $\pm 250 \mathrm{~mm}$, TOF $1 \pm 240 \mathrm{~mm}$ and $\mathrm{CKOV} \pm 200 \mathrm{~mm}$.

successfully cover the Good $\mu^{+}$configuration space of the beam at each beamline location. It should be noted that the Good $\mu^{+}$fraction of the total space of the beam at each location is significantly smaller than in both the mid-emittance and large-emittance beamlines. This is 
a result of the collimation. It should also be noted that, in this simulation we used, a beam consisting of $2 \times 10^{6} \pi^{+}$i.e. double the statistics that were used in analysis of the previous two beamlines. This was needed to increase the Good $\mu^{+}$statistics as the inclusion of the collimator significantly reduces the muon rate into the upstream tracker and consequently the Good $\mu^{+}$ rate as can be seen in Table 7.13. The rate reduces to between 7-8 times lower than the rate achieved by the previous two beamlines. This will require longer data runs with low emittance settings.

\begin{tabular}{|c|c|c|c|c|}
\hline Downstream position & LAHET & GEANT4 & MARS & Mean rate \\
\hline \hline TOF0 & 2.46 & 2.95 & 3.13 & $2.85 \pm 0.12$ \\
CKOV1 & 2.39 & 2.86 & 3.03 & $2.76 \pm 0.11$ \\
TOF1 & 0.99 & 1.18 & 1.26 & $1.14 \pm 0.05$ \\
Tracker 1 & 0.13 & 0.16 & 0.17 & $0.16 \pm 0.01$ \\
Good $\mu^{+}$ & 0.12 & 0.15 & 0.16 & $0.14 \pm 0.01$ \\
\hline
\end{tabular}

Table 7.13: Positive muon rates in $\mathrm{MHz}$ along the low emittance $200 \mathrm{MeV} / \mathrm{c}$ MICE beamline

The reduced rate beam does achieve the correct momentum in the tracker (see Figure 7.17) and has high purity as can be seen in Table 7.14. To conclude the analysis, one must again evaluate the emittance produced by this beamline. The evolution of the emittance along the beamline is shown in Figure 7.18 and does achieve the desired $1 \pi \mathrm{mm}$ mrad.

\begin{tabular}{|c|c|c|c|c|}
\hline Particle & TOF0 (\%) & CKOV (\%) & TOF1 (\%) & Tracker (\%) \\
\hline \hline$\mu^{+}$ & 98.30 & 98.96 & 99.61 & 100 \\
$\pi^{+}$ & 1.16 & 0.92 & 0.26 & 0 \\
$e^{+}$ & 0.08 & 0.08 & 0.13 & 0 \\
Proton & 0.45 & 0.04 & 0 & 0 \\
\hline
\end{tabular}

Table 7.14: Purity of $1 \pi, 200 \mathrm{MeV} / \mathrm{c}$ beamline sampled at each of the beamline detectors and upstream tracker

\subsection{A typical example of cooling in MICE}

The beamline designs shown in the previous sections will provide $200 \mathrm{MeV} / \mathrm{c}$ muon beams to the MICE experiment across the desired emittance range. MICE will then aim to show cooling of these beams at the $10 \%$ level by passing the beams through the components discussed in 


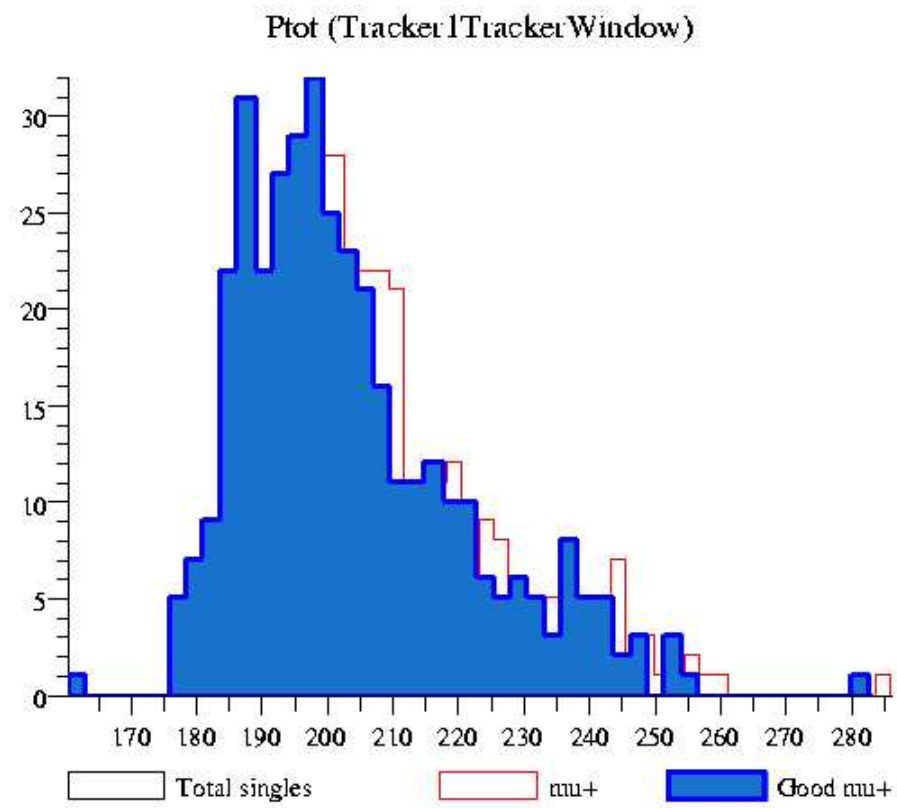

Figure 7.17: Ptot distribution at the entrance to the upstream MICE tracker for the CM14$1 \mathrm{pi}-200 \mathrm{MeV} / \mathrm{c}$ tune

Chapter 5. The cooling performance is outwith the scope of this thesis as the work carried out aims to provide the design requirements for the beamline. However, it is instructive to show a typical example of cooling in the MICE experiment. A simulation of the the full MICE Stage VI setup was created using the G4MICE software package, again discussed in Chapter 5. The simulation tracks a nominal $6.25 \pi \mathrm{mm} \mathrm{mrad}, 200 \mathrm{MeV} / \mathrm{c}$ muon beam from the upstream tracker to downstream tracker. This includes the 3 liquid hydrogen absorber mediums and both sets of R.F cavities and as such should provide the most accurate simulation of MICE performance available. Figure 7.19 shows the result of this simulation, plotted is the $4 \mathrm{D}$ normalised emittance (see Chapter 5) of the beam as it traverses the MICE experiment. The distance along the experiment is relative to the MICE coordinate system in which zero is defined to be the centre of the middle hydrogen absorber. The upstream tracker is located between approximately 6000 to $-4600 \mathrm{~mm}$ and the downstream tracker at approximately 4600 to $6000 \mathrm{~mm}$. It can be observed from the plot that this beam does cool at the $10 \%$ level as required. It will remain the responsibility of the MICE team to show this performance in practice for the beams delivered by the beamline. This will require minor alterations to the MICE magnet currents from the current default design values. 

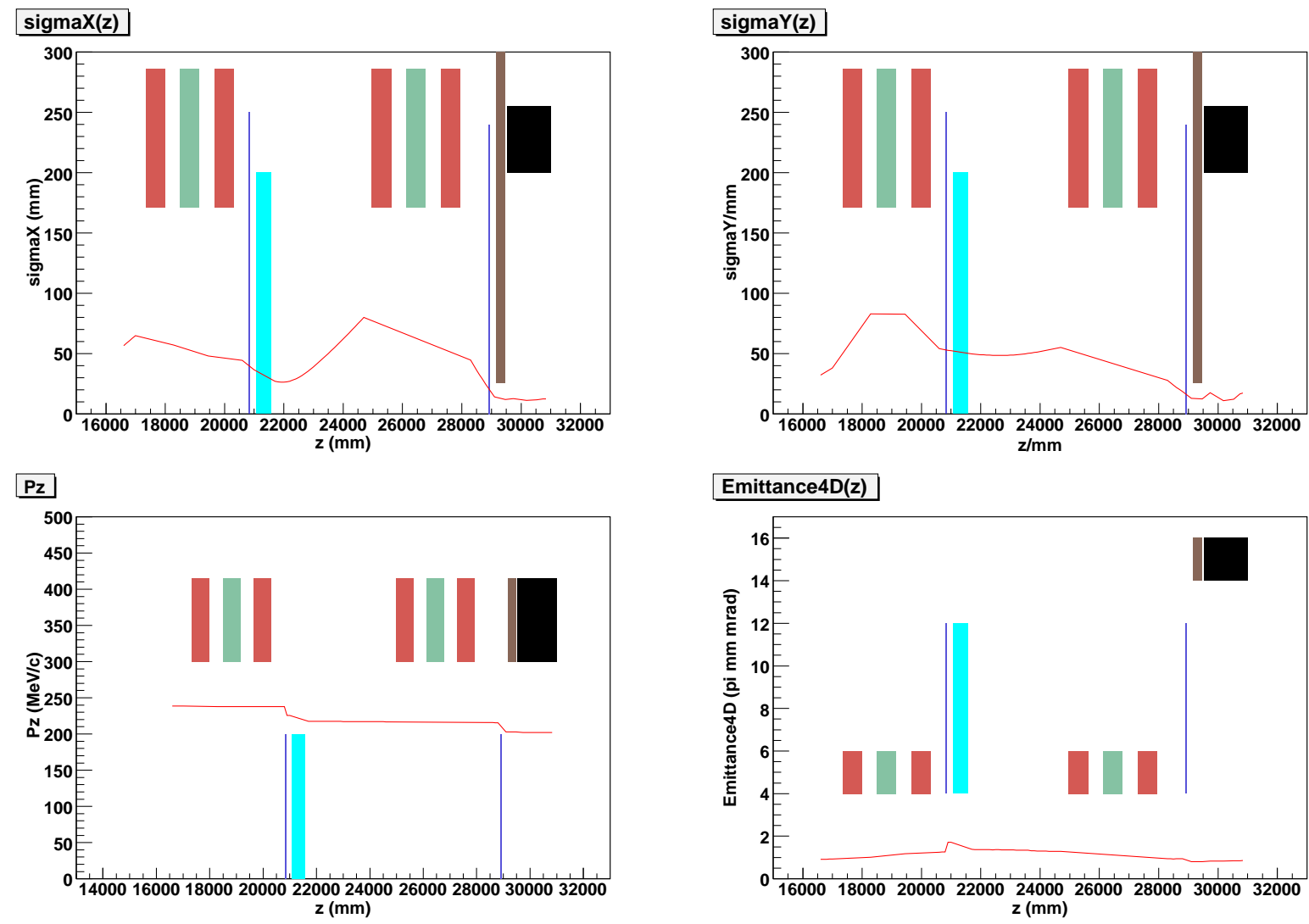

Figure 7.18: Results of GEANT4 implementation of the CM14- $1 \pi-200 \mathrm{MeV} / \mathrm{c}$ beamline optics tune. Plots show the evolution of the beam properties over the muon matching section. These properties are calculated for the reduced phase space volume with $215.31<E_{\mu}<252.2$ sampled at the first MICE tracker. Schematic representation of the beamline is indicated by different volumes on plots according to the following key: Matt red (horizontal focussing quad); Matt green (horizontal defocussing quad); Thin black (Iron shield); Thick black (tracker solenoid, only aperture correct); Blue (TOF0 and TOF1); Thick light blue (CKOV); Brown (Collimator)

\subsection{Conclusions}

This chapter has detailed design work and analysis of three accurately modelled muon beamlines that couple the MICE experiment to the ISIS synchrotron. It has been shown that optics solutions have been achieved that provide the baseline $200 \mathrm{MeV} / \mathrm{c}$ muons required by MICE to show cooling. The emittances of the three beamlines calculated in the pre-cooling tracker span the range of emittances required by MICE. It has been shown that the beamlines produced are compatible with the detector choices made by MICE and that the beams are very pure. For the large and mid emittance cases the optical lattices supply positively charged muons at a rate that is better than the baseline MICE value of $600 \mathrm{Good} \mu^{+}$per second. To produce a low emittance beam the beamline will require collimation. The design shown here does provide the correct 


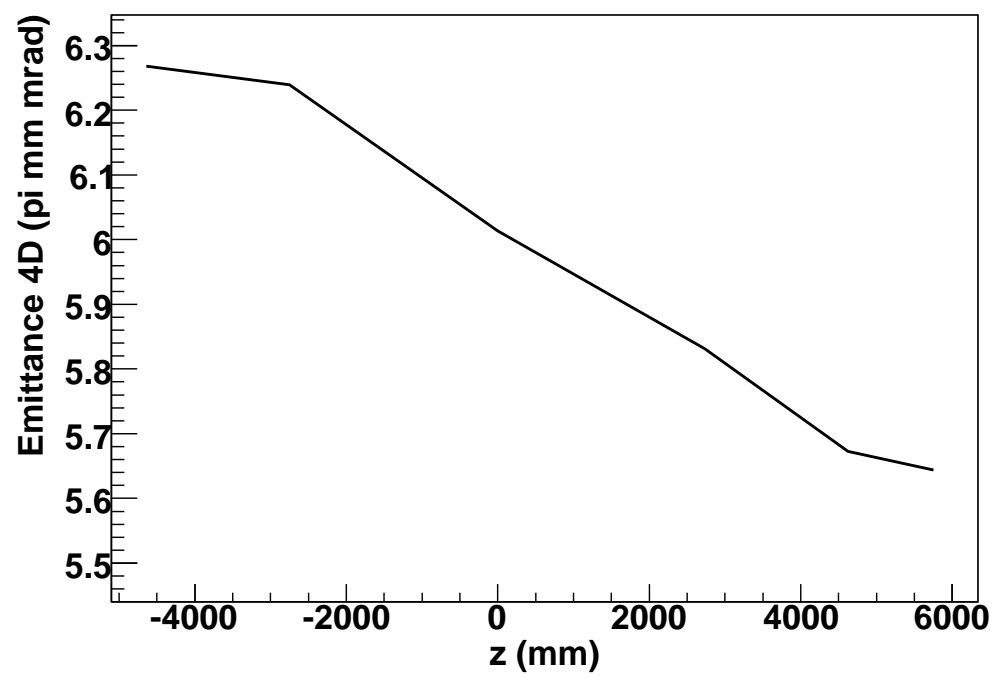

Figure 7.19: Cooling performance of a respresentative $6.25 \pi \mathrm{mm} \mathrm{mrad,} 200 \mathrm{MeV} / \mathrm{c}$ muon beam through MICE. The four-dimensional transverse emittance of the beam is shown as a function of length along the channel given in the MICE coordinate system where zero is defined to the the centre of the middle absorber.

emittance beam but at the expense of rate. This is not foreseen as a problem however as the planned MICE run schedule has sufficient scope to allow for longer running at low emittance settings. It has been shown that a representative $6.25 \pi \mathrm{mm}$ mrad beam is expected to cool when the full MICE Stage VI experiment starts data taking. 


\section{Chapter 8}

\section{Conclusions}

This thesis presents work focussed on essential R\&D towards the implementation of a viable Neutrino Factory. As elaborated in Chapters 1 and 2, the precision measurement of the neutrino mixing angle $\theta_{13}$ and possible probing of the $\mathrm{CP}$ violating phase will be extremely important to our understanding of the universe. The Neutrino Factory is the best facility envisaged to probe these fundamental parameters.

A key aspect of a Neutrino Factory will be the choice of proton driver and target configuration. The work presented in Chapter 3 investigates the optimal energy for such a proton driver impinging on a solid tantalum target. Studies of the optimal pion production yields into captureable phase space volumes are studied as are the performance of two candidate pion front-end capture channels. The comparison is made between pion production using GEANT4 and MARS standard particle interaction and tracking codes. In addition, within GEANT4 itself the results obtained by simulation using a number of hadronic production engines are also compared to each other and to MARS. Chapter 4 compares the predictions of the hadronic meson production in both codes to the only data available in this regime at the time., the results from the HARP experiment.

In addition to the requirement for an optimised proton driver and collection system it will be essential to cool the muon beam such that it can be successfully injected into an accelerator system. The MICE experiment (described in Chapter 5) aims to be a fully engineered cell of a cooling channel capable of measuring $10 \%$ cooling of a number of muon beams to good precision. This variable muon beam is provided by the MICE beamline which transports muons produced 
from decay of a tertiary pion beam produced from protons impinging on a titanium target. The proton source is the ISIS proton synchrotron housed in Oxfordshire, Great Britain. Chapter 6 details the construction and analysis of an experiment carried out in ISIS to measure key parameters of the beam/target interaction. Parameters with high impact on MICE performance which were previously unknown, for which assumptions had to be made were measured in this experiment. The results have established key relationships between ISIS beam loss and particle yields into the MICE beamline, verified simulations used to normalise beam rates and imaged the edge of the ISIS beam for the first time.

Finally, Chapter 7 presents the design and evaluation of the MICE beamline. The work presented details optics solutions for beamlines providing the baseline $200 \mathrm{MeV} / \mathrm{c} \mu^{+}$beam at three different emittances into the MICE experiment. The beams produced meet all the requirements on purity, rate and transverse configuration space. This work should enable MICE to run with $200 \mathrm{MeV} / \mathrm{c}$ muons beams over a range of emittances. It was also shown by running a full simulation of MICE that the experiment will cool at a $10 \%$ level for a respresentative 6.25 $\pi \mathrm{mm}$ mrad emittance beam, The work in this chapter has demonstrated that there are a range of optics solutions to the MICE beamline that will allow MICE to enable cooling performance to be quantified over a number of phase spaces.

To conclude, it is useful to comment on future extensions to the work shown in this thesis. Firstly in relation to studies of Neutrino Factory targets. The results of the HARP experiment are currently being released for a number of target materials. It will be important that once the HARP data is incorporated into GEANT4 and MARS the studies here are repeated. This should bring convergence between code and data in this notoriously difficult regime. Extensions to the work presented on the MICE experiment should first include an increase in the number of optics solutions for the beamline i.e. the extension of the lattices shown to provide 140 and 240 $\mathrm{MeV} / \mathrm{c}$ beams into MICE. Also, at the time of writing the physical beamline is being constructed according to the specifications of this design. It will be important to use this to study further particle production from the target using the upstream particle identification detectors. This should start sometime around the end of 2007. 


\section{Bibliography}

[1] W. Pauli. Letter proposing the neutrino (1930). Physics Today, Letter published, September 1978 .

[2] F.A Scott. Energy Spectrum of the Beta-Rays of Radium E. 1935. Phys. Rev. 48391.

[3] F.Reines et al. Science 124 103, 1956.

[4] W.-M. Yao, C. Amsler, D. Asner, R.M. Barnett, J. Beringer, P.R. Burchat, C.D. Carone, C. Caso, O. Dahl, G. D’Ambrosio, A. DeGouvea, M. Doser, S. Eidelman, J.L. Feng, T. Gherghetta, M. Goodman, C. Grab, D.E. Groom, A. Gurtu, K. Hagiwara, K.G. Hayes, J.J. Hernández-Rey, K. Hikasa, H. Jawahery, C. Kolda, Kwon Y., M.L. Mangano, A.V. Manohar, A. Masoni, R. Miquel, K. Mönig, H. Murayama, K. Nakamura, S. Navas, K.A. Olive, L. Pape, C. Patrignani, A. Piepke, G. Punzi, G. Raffelt, J.G. Smith, M. Tanabashi, J. Terning, N.A. Törnqvist, T.G. Trippe, P. Vogel, T. Watari, C.G. Wohl, R.L. Workman, P.A. Zyla, B. Armstrong, G. Harper, V.S. Lugovsky, P. Schaffner, M. Artuso, K.S. Babu, H.R. Band, E. Barberio, M. Battaglia, H. Bichsel, O. Biebel, P. Bloch, E. Blucher, R.N. Cahn, D. Casper, A. Cattai, A. Ceccucci, D. Chakraborty, R.S. Chivukula, G. Cowan, T. Damour, T. DeGrand, K. Desler, M.A. Dobbs, M. Drees, A. Edwards, D.A. Edwards, V.D. Elvira, J. Erler, V.V. Ezhela, W. Fetscher, B.D. Fields, B. Foster, D. Froidevaux, T.K. Gaisser, L. Garren, H.-J. Gerber, G. Gerbier, L. Gibbons, F.J. Gilman, G.F. Giudice, A.V. Gritsan, M. Grünewald, H.E. Haber, C. Hagmann, I. Hinchliffe, A. Höcker, P. IgoKemenes, J.D. Jackson, K.F. Johnson, D. Karlen, B. Kayser, D. Kirkby, S.R. Klein, K. Kleinknecht, I.G. Knowles, R.V. Kowalewski, P. Kreitz, B. Krusche, Yu.V. Kuyanov, O. Lahav, P. Langacker, A. Liddle, Z. Ligeti, T.M. Liss, L. Littenberg, L. Liu, K.S. Lugovsky, S.B. Lugovsky, T. Mannel, D.M. Manley, W.J. Marciano, A.D. Martin, D. Milstead, 
M. Narain, P. Nason, Y. Nir, J.A. Peacock, S.A. Prell, A. Quadt, S. Raby, B.N. Ratcliff, E.A. Razuvaev, B. Renk, P. Richardson, S. Roesler, G. Rolandi, M.T. Ronan, L.J. Rosenberg, C.T. Sachrajda, S. Sarkar, M. Schmitt, O. Schneider, D. Scott, T. Sjöstrand, G.F. Smoot, P. Sokolsky, S. Spanier, H. Spieler, A. Stahl, T. Stanev, R.E. Streitmatter, T. Sumiyoshi, N.P. Tkachenko, G.H. Trilling, G. Valencia, K. van Bibber, M.G. Vincter, D.R. Ward, B.R. Webber, J.D. Wells, M. Whalley, L. Wolfenstein, J. Womersley, C.L. Woody, A. Yamamoto, O.V. Zenin, J. Zhang, and R.-Y. Zhu. Review of Particle Physics. Journal of Physics G, 33:1+, 2006.

[5] M. Apollonio et al. Oscillation Physics with a Neutrino Factory, 2002. http://www. citebase. org/abstract?id=oai : arXiv .org: hep-ph/0210192.

[6] B Kayser. Neutrino oscillations. In PHENO2005, University of Wisconsin-Madison, May 2005.

[7] L. Wolfenstein. Neutrino oscillations in matter. Phys. Rev. D, 17(9):2369-2374, May 1978.

[8] S. P. Mikheev and A. Yu. Smirnov. Resonance enhancement of oscillations in matter and solar neutrino spectroscopy. Sov. J. Nucl. Phys., 42:913-917, 1985.

[9] S. Fukuda and others [Super-Kamiokande Collaboration]. Solar Neutrino Data Covering Solar Cycle 22. Nucl. Instrum. Meth., A501:418-462, 2003.

[10] Y. Ashie et al. A measurement of atmospheric neutrino oscillation parameters by SuperKamiokande I. Phys. Rev., D71:112005, 2005.

[11] Y. Ashie et al. Evidence for an oscillatory signature in atmospheric neutrino oscillation. Phys. Rev. Lett., 93:101801, 2004.

[12] http://www/sns.ias.edu/ jnb/.

[13] First real time detection of ${ }^{7} B e$ solar neutrinos by Borexino. 2007.

[14] R. Davis Jr. A review of the homestake solar neutrino experiment. Progress in Particle and Nuclear Physics, 32:13-32, 1994. 
[15] John N. Bahcall, Aldo M. Serenelli, and Sarbani Basu. 10,000 Standard Solar Models: a Monte Carlo Simulation. 2005. http://www.citebase.org/abstract?id=oai : arXiv . org: astro-ph/0511337.

[16] SAGE Collaboration: J.N. Abdurashitov. Measurement of the Solar Neutrino Capture Rate by the Russian-American Gallium Solar Neutrino Experiment During One Half of the 22-Year Cycle of Solar Activity. ZH.EKSP.TEOR.FIZ., 122:211, 2002.

[17] http://www.lngs.infn.it/site/exppro/gallex/Gallexres.htm.

[18] J. Hosaka et al. Solar neutrino measurements in Super-Kamiokande-I. Phys. Rev., D73:112001, 2006.

[19] Image from http://kamland.lbl.gov/FiguresPlots/.

[20] B. Aharmim et al. Electron energy spectra, fluxes, and day-night asymmetries of B-8 solar neutrinos from the 391-day salt phase SNO data set. Phys. Rev., C72:055502, 2005.

[21] T. Araki et al. Measurement of neutrino oscillation with KamLAND: Evidence of spectral distortion. Phys. Rev. Lett., 94:081801, 2005.

[22] M. Apollonio et al. Limits on neutrino oscillations from the CHOOZ experiment. Phys. Lett., B466:415-430, 1999.

[23] WHITE PAPER REPORT on Using Nuclear Reators to Search for a value of $\theta_{13} .2004$. neutrinooscillation.org/white.html.

[24] K. Eguchi et al. A high sensitivity search for anti-nu/e's from the sun and other sources at KamLAND. Phys. Rev. Lett., 92:071301, 2004.

[25] K. Eguchi et al. First results from KamLAND: Evidence for reactor anti- neutrino disappearance. Phys. Rev. Lett., 90:021802, 2003.

[26] LSND Collaboration: C. Athanassopoulos, L. B. Auerbach, R. L. Burman, D. O. Caldwell, E. D. Church, I. Cohen, J. B. Donahue, A. Fazely, F. J. Federspiel, G. T. Garvey, R. M. Gunasingha, R. Imlay, K. Johnston, H. J. Kim, W. C. Louis, R. Majkic, K. McIlhany, W. Metcalf, G. B. Mills, R. A. Reeder, V. Sandberg, D. Smith, I. Stancu, W. Strossman, 
R. Tayloe, G. J. VanDalen, W. Vernon, N. Wadia, J. Waltz, D. H. White, D. Works, Y. Xiao, and S. Yellin. Evidence for nu_mu -> nu_e Neutrino Oscillations from LSND. Physical Review Letters, 81:1774, 1998.

[27] A. A. Aguilar-Arevalo et al. A Search for Electron Neutrino Appearance at the $\Delta m^{2} \sim 1$ $\mathrm{eV}^{2}$ Scale. arXiv:0704.1500 [hep-ex], 2007.

[28] D. G. Michael et al. Observation of muon neutrino disappearance with the MINOS detectors and the NuMI neutrino beam. Phys. Rev. Lett., 97:191801, 2006.

[29] General description of the CERN project for a neutrino beam to Gran Sasso. CERN AC Note (2000-03), 2000-2003.

[30] Y. Itow et al. The JHF-Kamioka neutrino project. 2001.

[31] Carl H. Albright et al. The neutrino factory and beta beam experiments and development. 2004 .

[32] P Zucchelli. A novel concept for a neutrino factory: the beta-beam. Phys. Lett. B, $532: 166-172,2002$.

[33] M. Benedikt. Beta-Beam R \& D in europe. Nucl. Phys. B, 149:54-59, 2005.

[34] The Eurisol Report. 2003. http://www.ganil.fr/eurisol/Final_Report/ EURISOL-REPORT . pdf.

[35] http://hitoshi.berkeley.edu/neutrino/.

[36] D. G. Koshkarev. Proposal for a Decay Ring to Produce Intense Secondary Particle Beams at the SPS. CERN/ISR-DI/74-62.

[37] D Cline and D Neuffer. A muon storage ring for neutrino oscillation experiments. AIP Conf. Proc., 68, 1980.

[38] W Lee et al. A muon storage ring for neutrino oscillation experiments. FNAL proposal P860.

[39] S. Geer. Neutrino beams from muon storage rings: Characteristics and physics potential. Phys. Rev., D57:6989-6997, 1998. 
[40] H Kirk et al. A proposal to the ISOLDE and Neutron Time-of-Flight Experiments Committee: Studies of a Target System for a 4-MW, $24 \mathrm{GeV}$ proton Beam. CERN-INTC2004-016, 2006.

[41] S. Ozaki, R. Palmer, M. Zisman, and J. Gallardo. Feasibility Study-II of a Muon-Based Neutrino Source. BNL-52623, 2001.

[42] International scoping study of a future Neutino Facory and Super-beam facility. 2007. http://www.hep.ph.ic.ac.uk/iss/.

[43] S. Rigolin. Physics reach of beta-beams and nu-factories: The problem of degeneracies. Nucl. Phys. Proc. Suppl., 155:33-37, 2006.

[44] K. S. McFarland. Short-baseline opportunities and challenges at a neutrino factory. Nucl. Instrum. Meth., A451:218-228, 2000.

[45] P. Huber, M. Lindner, M. Rolinec, and W. Winter. Optimization of a neutrino factory oscillation experiment. Phys. Rev., D74:073003, 2006.

[46] A. Cervera et al. Golden measurements at a neutrino factory. Nucl. Phys., B579:17-55, 2000.

[47] http://www.isis.rl.ac.uk.

[48] C.R Prior. General Issues for a Multi-MegaWatt Proton Driver for a Neutrino Factory. Nucl. Phys. B (Proc. Suppl.), 155:293-294, 2006.

[49] J Chen et al. Mechanical properties of pure tantalum after $800 \mathrm{MeV}$ proton irradiation. Nucl. Mater., 298:248-254, 2001.

[50] J.R.J Bennett et al. LS-DYNA calculations of shocks in solids. Nucl. Phys. B (Proc. Suppl.), 155:318-320, 2006.

[51] S. Agostinelli, J. Allison, K. Amako, J. Apostolakis, H. Araujo, P. Arce, M. Asai, D. Axen, S. Banerjee, G. Barrand, F. Behner, L. Bellagamba, J. Boudreau, L. Broglia, A. Brunengo, H. Burkhardt, S. Chauvie, J. Chuma, R. Chytracek, G. Cooperman, G. Cosmo, P. Degtyarenko, A. Dell'acqua, G. Depaola, D. Dietrich, R. Enami, A. Feliciello, C. Fergu- 
son, H. Fesefeldt, G. Folger, F. Foppiano, A. Forti, S. Garelli, S. Giani, R. Giannitrapani, D. Gibin, Gomez, I. Gonzalez, Gracia, G. Greeniaus, W. Greiner, V. Grichine, A. Grossheim, S. Guatelli, P. Gumplinger, R. Hamatsu, K. Hashimoto, H. Hasui, A. Heikkinen, A. Howard, V. Ivanchenko, A. Johnson, F. W. Jones, J. Kallenbach, N. Kanaya, M. Kawabata, Y. Kawabata, M. Kawaguti, S. Kelner, P. Kent, A. Kimura, T. Kodama, R. Kokoulin, M. Kossov, H. Kurashige, E. Lamanna, T. Lampen, V. Lara, V. Lefebure, F. Lei, M. Liendl, W. Lockman, F. Longo, S. Magni, M. Maire, E. Medernach, K. Minamimoto, Mora, Y. Morita, K. Murakami, M. Nagamatu, R. Nartallo, P. Nieminen, T. Nishimura, K. Ohtsubo, M. Okamura, S. O’Neale, Y. Oohata, K. Paech, J. Perl, A. Pfeiffer, M. G. Pia, F. Ranjard, A. Rybin, S. Sadilov, E. Di Salvo, G. Santin, T. Sasaki, N. Savvas, Y. Sawada, S. Scherer, S. Sei, V. Sirotenko, D. Smith, N. Starkov, H. Stoecker, J. Sulkimo, M. Takahata, S. Tanaka, E. Tcherniaev, Safai, M. Tropeano, P. Truscott, H. Uno, L. Urban, P. Urban, M. Verderi, A. Walkden, W. Wander, H. Weber, J. P. Wellisch, T. Wenaus, D. C. Williams, D. Wright, T. Yamada, H. Yoshida, and D. Zschiesche. Geant4-a simulation toolkit. Nuclear Instruments and Methods in Physics Research Section A: Accelerators, Spectrometers, Detectors and Associated Equipment, 506(3):250-303, July 2003.

[52] N.V Mokhov. MARS Code System. http://www-ap.fnal.gov/MARS/.

[53] HARP Collaboration. Proposal to study hadron production for the neutrino factory and for the atmospheric neutrino flux. 1999. http://harp.web.cern.ch/harp/Documents/ Proposal/hpeproposal.pdf.

[54] http://cepa.fnal.gov/psm/geant4/G4BeamTools/.

[55] http://fermitools.fnal.gov/abstracts/histoscope/abstract.html.

[56] S.J Brooks and K.A Walaron. Computed Pion Yields from a Tantalum Rod Target. Presentation given at the 7th International Neutrino Factory, Superbeams and Betabeams Conference (NUFACT'05) Frascati, Italy, 2005.

[57] S.J Brooks. Quantitive Optimisation Studies of the Muon Front-End for a Neutrino Factory. Proc. European Particle Acceleration Conference EPAC'04, 2004. 
[58] S.J Brooks and K.A Walaron. Computed Pion Yields from a Tantalum Rod Target, Comparing MARS15 and GEANT4. Nucl. Phys. B (Proc. Suppl.), 155:295-296, 2006.

[59] L Howlett. Simulation and Correction of Cross Talk in the HARP Time Projection Chamber, PhD Thesis, University of Sheffield.

[60] M. Bogomilov et al. The HARP RPC time-of-flight system. Nucl. Instrum. Meth., A508:152-158, 2003.

[61] M. Anfreville et al. The drift chambers of the Nomad experiment. Nucl. Instrum. Meth., A481:339-364, 2002.

[62] M. G. Catanesi et al. Particle identification algorithms for the HARP forward spectrometer. Nucl. Instrum. Meth., A572:899-921, 2007.

[63] HARP Collaboration. Measurement of the production cross-section of positive pions in p-Al collisions at $12.9 \mathrm{GeV} / \mathrm{c}$. Nuclear Physics B, 732:1, 2006.

[64] Measurement of the production cross-section of positive pions in the collision of $8.9 \mathrm{GeV} / \mathrm{c}$ protons on beryllium. Eur. Phys. J., C52:29-53, 2007.

[65] M. G. Catanesi et al. Measurement of the production of charged pions by protons on a tantalum target. 2007.

[66] R Brun et al. ROOT:An Object-Orientated Data Analysis Framework. http://root. cern.ch/.

[67] http://www.physics.ox.ac.uk/design/mice_page.htm.

[68] G Penn. Beam Envelope Equations in a Solenoidal Field. Muon Collider Note 71.

[69] Univ. of New Mexico Prof. Stanley Humphries, Emeritus Professor. Introduction to charged particle beams. http://www.fieldp.com/cpb/cpb.html.

[70] S.Y Lee. Accelerator Physics, 2nd Ed. Published by World Scientific 2004.

[71] R. B. Palmer. Muon Collider: Introduction and status. AIP Conf. Proc., 435:11-36, 1998.

[72] D Neuffer. Muon transverse ionization cooling: Stochastic approach. Part. Acc., (14):7590, 1983. 
[73] J Cobb. Presentation at the 14th MICE Collab. meeting, Osaka, Japan, 2004.

[74] J Buon. Beam phase space and emittance. CERN Accelerator School 5, Jyvaeskylae, pages 89-116, 1994.

[75] W.M Yao et al. Particle Physics Data Group, Review of passage of particles through matter. J. Phys., G(33):1, 2006.

[76] http://www.mice.iit.edu/software/software.html.

[77] The MICE collab. Muon Ionisation Cooling Experiment, Technical Reference Document. http://www.isis.rl.ac.uk/accelerator/MICE/TR/MICE_Tech_ref.html.

[78] The MICE Collab. Muon Ionisation Cooling Experiment Proposal. www.hep.ph.ic.ac. uk/ longkr/MICE/SOI-Prop/2003-04-28/MICE-UK-proposal .pdf.

[79] Ed. Bross, Ruchti, and Wayne. Proc. SCIFI97, Conference on Scintillating and Fibre Detectors, University of Notre Dame, November 1997. World Scientific, Singapore, 1998.

[80] A Khan et al. MICE scintillating-fibre tracker. MICE note 90.

[81] The KLOE Collab. The KLOE Detector Technical Proposal. LNF-92/019, 1992.

[82] U Bravar. Coil currents in MICE. MICE note 88.

[83] C.M Warsop. Studies of Beam Loss Control on the ISIS Synchrotron. Proc. 9th European Particle Accelerator Conference (EPAC2004), Lucerne, 2004.

[84] C Booth et al. MICE Technical Reference Document: Target Section. http://www . isis . rl.ac.uk/accelerator/MICE/TR/MICE_Tech_ref.html.

[85] D. Attwood, P. Bell, S. Bull, T. McMahon, J. Wilson, R. Fernow, P. Gruber, A. Jamdagni, K. Long, E. McKigney, P. Savage, M. Curtis-Rouse, T. R. Edgecock, M. Ellis, J. Lidbury, W. Murray, P. Norton, K. Peach, K. Ishida, Y. Matsuda, K. Nagamine, S. Nakamura, G. M. Marshall, S. Benveniste, D. Cline, Y. Fukui, K. Lee, Y. Pischalnikov, S. Holmes, and A. Bogacz. The scattering of muons in low-Z materials. Nuclear Instruments and Methods in Physics Research B, 251:41-55, September 2006.

[86] http://www.tek.com/site/ps/0, ,3G-15314-INTRO_EN, 00.html. 
[87] http://www.lecroy.com/tm/products/scopes/WaveRunner_6000/WaveRunner_ datasheet.pdf.

[88] G. Knoll. Radiation Detection and Measurement, 3rd Ed. Published by John Wiley and Sons, Inc. 2000.

[89] L Howlett. Analysis of Target Beam Test Data. MICE note 165.

[90] Di Wright (ISIS). Personal correspondence.

[91] Target Group Paul Smith Univ. of Sheffield. Personal correspondence.

[92] K. Tilley, D. J. Adams, P. Drumm, T. J. Roberts, and K. A. Walaron. Design and expected performance of the muon beamline for the Muon Ionisation Cooling Experiment. Prepared for European Particle Accelerator Conference (EPAC 06), Edinburgh, Scotland, 26-30 Jun 2006.

[93] http://www-xdiv.lanl.gov/XCI/PROJECTS/LCS/index.html.

[94] http://www.opengl.org/documentation/.

[95] TJ Roberts. MICE Beamline Magnet Drawings. MICE note 65.

[96] G H Eaton. Muon Facilities and Experiments; Now and in the New Millenium. CCLRC Technical Report RAL-TR-1998-019.

[97] W Lau. Diffuser Report. Presentation at the 14th MICE Collab. meeting, Osaka, Japan.

[98] http://www.aps.anl.gov/epics.

[99] D.C Carey. Third-Order TRANSPORT with MAD Input A Computer Program for Designing Charged Particle Beam Transport Systems. FERMILAB-Pub-98/310.

[100] K.L. Brown, Ch Iselin, and D.C Carey. Decay Turtle. CERN 74-2, 1974.

[101] R Palmer. Accelerator physics. NUFACT04 Summer Institute, Tokyo, Japan, 2004.

[102] http://www.vectorfields.com/content/view/27/50/.

[103] K. Halbach. A magnetic field clamp with beneficial saturation effects. Nucl. Instrum. Meth., 119:327-328, 1974. 